\title{
Synthesis, Characterisation and Functionalisation of Magnetic Nanoparticles for Biomedical Applications
}

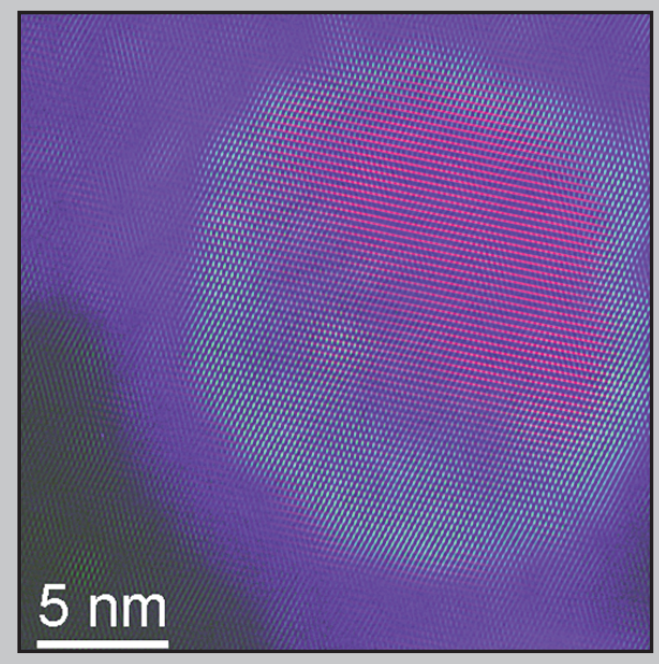

Rodrigo Fernández-Pacheco Chicón 

Synthesis, Characterisation and Functionalisation of Magnetic Nanoparticles for Biomedical Applications 


\section{Colección de Estudios de Física}

\section{Vol. 75}

Esta colección recoge las tesis presentadas en el Departamento de Física de la Materia Condensada de la Universidad de Zaragoza desde su constitución en 1987. 


\section{Synthesis, Characterisation and}

Functionalisation of Magnetic Nanoparticles for Biomedical Applications

Rodrigo Fernández-Pacheco Chicón

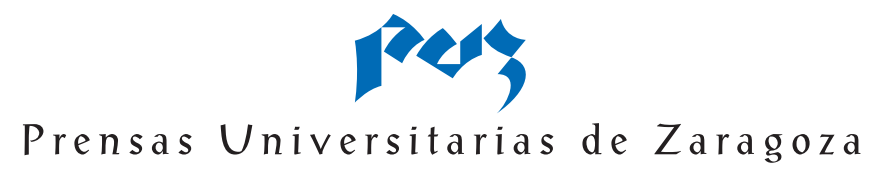




\section{FICHA CATALOGRÁFICA}

\section{FERNÁNDEZ-PACHECO CHICÓN, Rodrigo}

Synthesis, Characterisation and Functionalisation of Magnetic Nanoparticles for Biomedical Applications / Rodrigo Fernández-Pacheco Chicón. — Zaragoza : Prensas Universitarias de Zaragoza, 2008

XII, 222 p. ; 24 cm. - (Colección de Estudios de Física ; 75)

Tesis-Universidad de Zaragoza

ISBN 978-84-92521-02-9

1. Magnetismo-Tesis doctorales. 2. Nanotecnología-Tesis doctorales. 3. Medicina nuclear-Tesis doctorales. I. Universidad de Zaragoza. II. Título. III. Serie: Colección de Estudios de Física (Prensas Universitarias de Zaragoza) ; 75

537.6:61(043.2)

620.22:61(043.2)

616-073(043.2)

No está permitida la reproducción total o parcial de este libro, ni su tratamiento informático, ni la transmisión de ninguna forma o por cualquier medio, ya sea electrónico, mecánico, por fotocopia, por registro u otros métodos, ni su préstamo, alquiler o cualquier forma de cesión de uso del ejemplar, sin el permiso previo y por escrito de los titulares del Copyright.

(C) Rodrigo Fernández-Pacheco Chicón

(C) De la presente edición, Prensas Universitarias de Zaragoza $1 .^{a}$ edición, 2008

Prensas Universitarias de Zaragoza. Edificio de Ciencias Geológicas, c/ Pedro Cerbuna, 12, 50009 Zaragoza, España. Tel.: 976761 330. Fax: 976761063

puz@unizar.es http://puz.unizar.es

Impreso en España

Imprime: Servicio de Publicaciones. Universidad de Zaragoza

D.L.: Z-2866/2008 


\section{Index}

Preface

1 Introduction ........................................................................................................ 7

$1.1 \quad$ Magnetism at the nanoscale ............................................................ 7

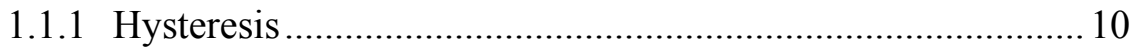

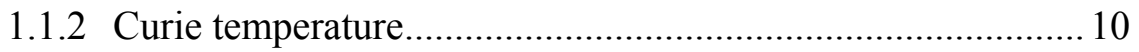

1.1.3 Magnetic Anisotropy …...................................................... 11

1.1.4 Coercivity of small particles ................................................ 15

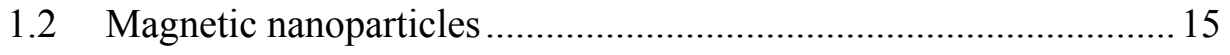

1.2.1 Top-down Vs Bottom-up methods of synthesis ..................... 17

1.2.2 Core-shell particles .............................................................. 19

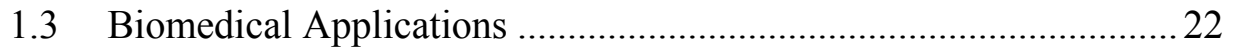

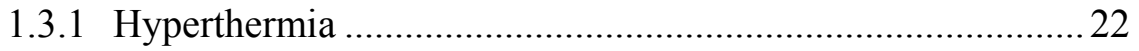

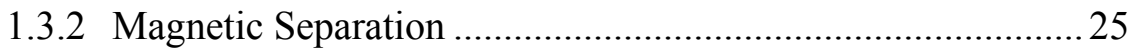

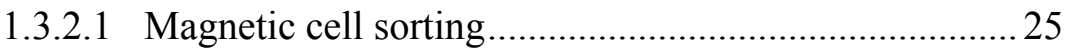

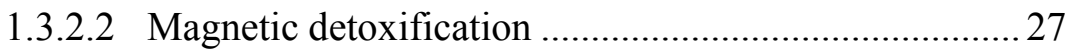

1.3.2.3 Separation and Magnetic biosensors.............................. 28

1.3.3 Magnetic Resonance Imaging (MRI) .................................... 30

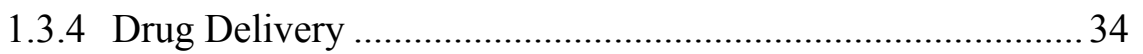

1.3.4.1 Magnetic carriers for local drug delivery ....................... 34

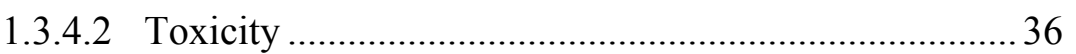

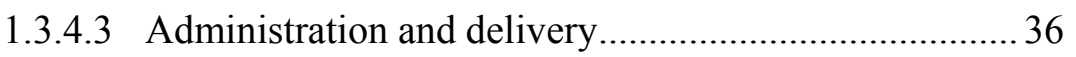

1.3.4.4 Intravenous administration............................................... 38 


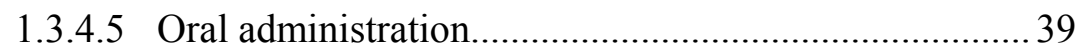

1.3.4.6 Subcutaneous or direct injection into the tumour. ......... 39

1.3.4.7 External magnets and magnetic implants...................... 40

1.3.4.8 Non-magnetic delivery systems ................................... 41

2 Materials and Methods ..................................................................................43

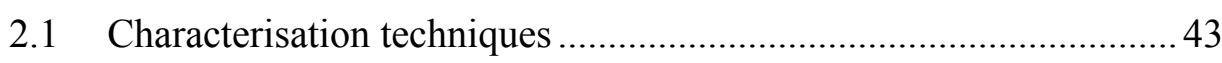

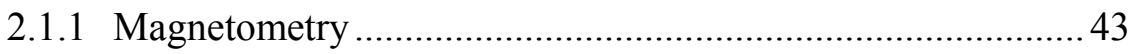

2.1.2 Scanning Electron Microscopy (SEM) .................................. 44

2.1.3 Transmission Electron Microscopy (TEM) ............................ 45

2.1.4 Fourier Transformed Infra-Red Spectroscopy (FTIR)............ 47

2.1.5 Ultra-Violet Visible Spectroscopy (UV-VIS) ......................... 48

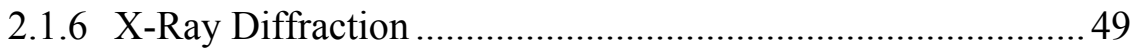

2.1.7 X-Ray Photoelectron Spectroscopy (XPS) .............................5 50

2.1.8 Dynamic Light Scattering (DLS) .......................................... 52

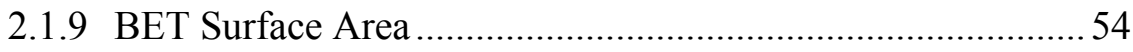

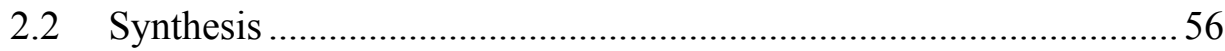

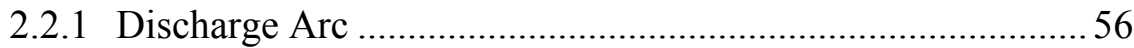

2.2.1.1 Iron Encapsulated in Carbon. Fe@C ............................56

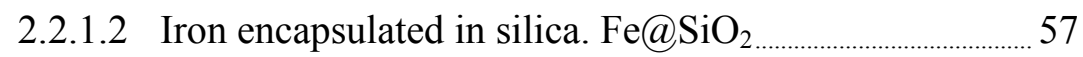

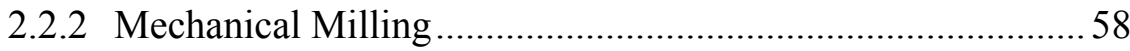

2.2.2.1 Magnetite embedded in a Zeolite matrix. $\mathrm{Fe}_{3} \mathrm{O}_{4} @ \mathrm{ZY} . .58$

2.2.3 Co-precipitation and sol-gel method.......................................59

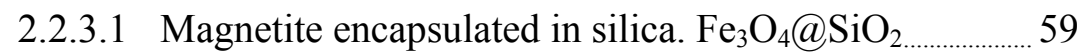

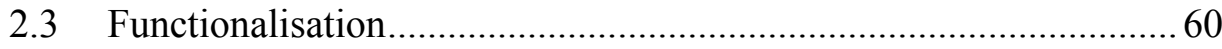

2.3.1 Surface Functionalisation: chemical coupling ......................... 60

2.3.1.1 Functionalisation with amine groups ........................... 60

2.3.1.2 Functionalisation with carboxyl groups ........................ 60

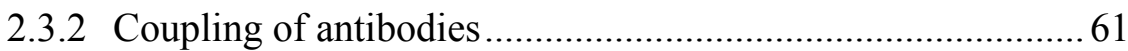




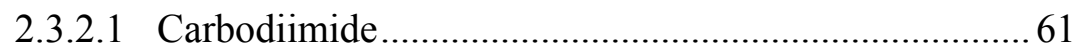

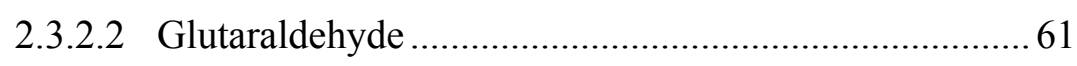

2.3.2.3 Verification of the antibody coupling .......................... 62

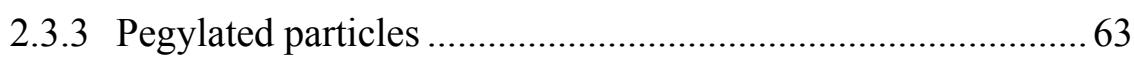

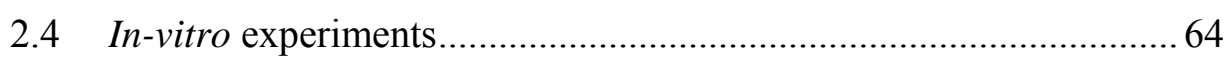

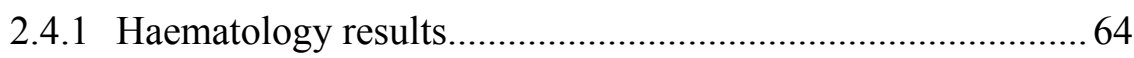

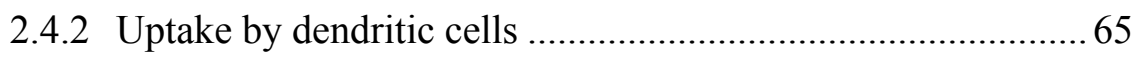

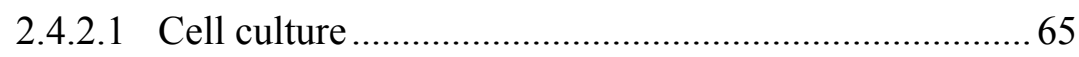

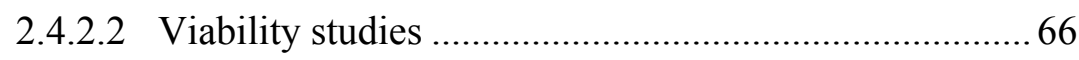

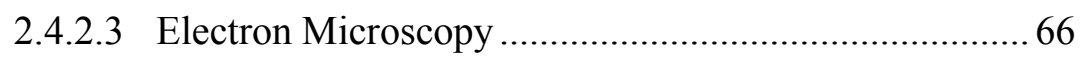

2.4.3 Kinetics studies of drug adsorption and release....................... 66

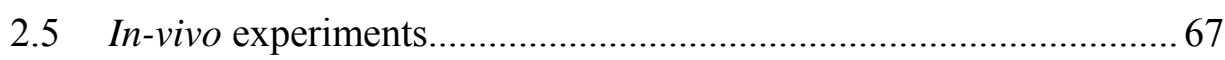

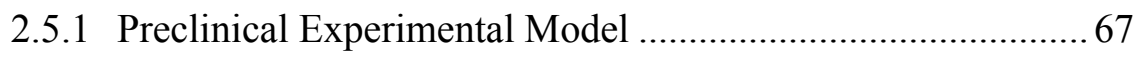

2.5.2 Intravenous administration of the particles.............................. 69

2.5.3 Direct injection into the target organ ....................................... 69

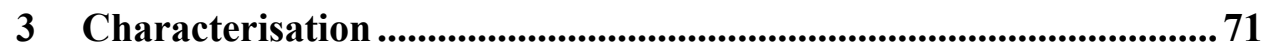

3.1 Iron coated by carbon. Fe@C ..................................................... 71

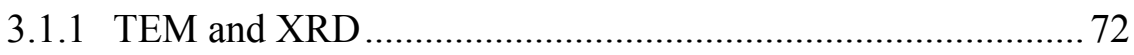

3.1.2 Dynamic Light Scattering. Estimation of size ........................ 76

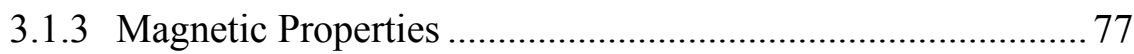

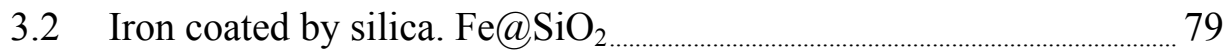

3.2.1 Magnetic Properties ............................................................. 79

3.2.2 Transmission Electron Microscopy ……………………........ 80

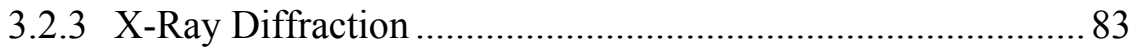

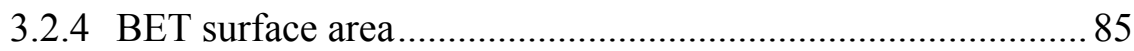

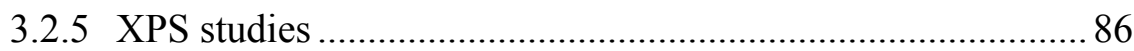

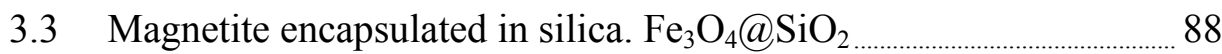

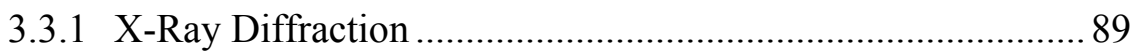


3.3.2 $\mathrm{N}_{2}$ adsorption/desorption isotherms .................................... 90

3.3.3 Transmission Electron Microscopy ....................................... 91

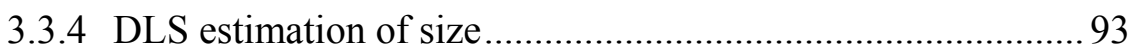

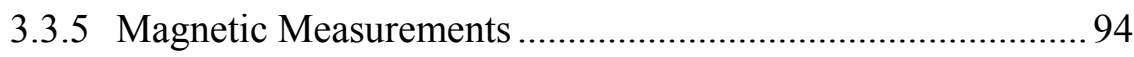

3.4 Magnetite embedded in a zeolite matrix. $\mathrm{Fe}_{3} \mathrm{O}_{4} @ \mathrm{ZY}$...................... 97

3.4.1 SEM and TEM ................................................................... 97

3.4.2 X-ray Diffraction ............................................................ 100

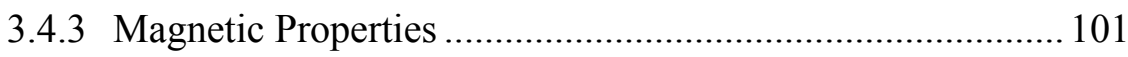

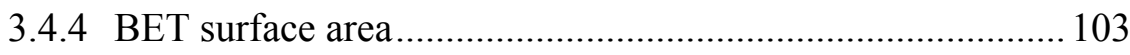

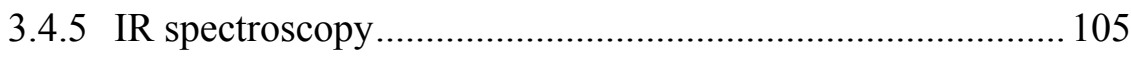

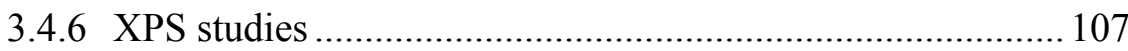

Appendix: Magnetic saturation and size for different nanomaterials...... 109

4 Functionalisation ................................................................................... 111

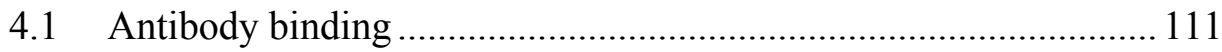

4.1.1 Functionalisation with carboxyl and amino groups.............. 113

4.1.2 XPS characterisation of functionalised surfaces.................. 114

4.1.3 Binding to the antibody anti-hCG..................................... 117

4.2 Coating with Serum Albumin and PEG......................................... 122

4.2.1 Bovine Serum Albumin coating ........................................ 125

4.2.2 Poly(ethyleneglycol) coating .............................................. 126

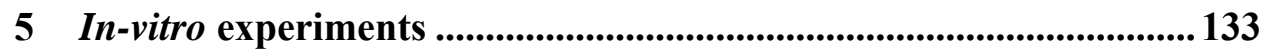

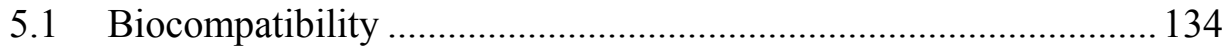

5.2 Cell uptake of nanoparticles ..................................................... 138

5.3 Drug adsorption and release studies ........................................... 146

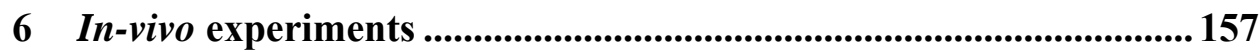




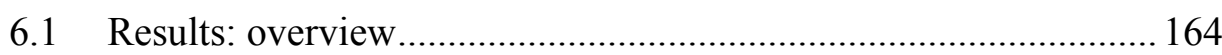

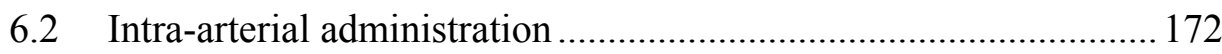

6.3 Ultra-sound scan-assisted injection of the particles....................... 174

6.4 Functionalised Nanoparticles...................................................... 175

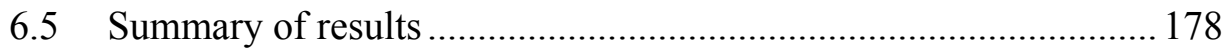

Summary and Conclusions ................................................................183

List of abbreviations ............................................................................. 187

Bibliography .............................................................................................................. 191 



\section{Preface}

Nanotechnology is a relatively new interdisciplinary field to which much attention has been paid for the last years. It involves researchers from very different areas: Chemistry, Physics, Biology, Biochemistry, Chemical Engineering, Materials Science or Medicine. In the interface of all these disciplines lay the possibility to tackle new challenges, unthinkable a few years ago [Klabunde2001].

Nanoscience has opened many possibilities in most technology areas, unreachable so far. It is devoted to the studies of phenomena at the nanoscale, that is, the limit "where the smallest man-made devices meet the atoms and molecules of the natural world" [Wong1999]. Nanoscience is based on the fabrication and characterization of nanostructured, or nanophase systems. These can be threedimensional: nanoparticles or nanospheres, two-dimensional: thin films, or onedimensional: quantum dots. The nanoscale regime is a very special point in the length scale, at which the classical laws of physics are not suitable for the explanation of many phenomena, so quantum approaches are needed. Significant changes in the chemical and physical properties of materials take place at the limit at which the interactions correlation length (electrical, magnetic, crystalline...) is of the same order of magnitude of the system size. That opens new possibilities for the development of smart new functional materials, like improved catalysts, polymers, ceramics, tissues, solid state medicines or drug carriers.

Nanoparticles are the most promising nanostructured systems for the application in nanotechnology. Among their main applications are:

- Environment: small particles are used in decontamination of water, by oxidation and reduction of pollutants. The high reactivity of some iron or zinc fine 
powders towards chlorocarbon compounds is also used for the purification of water. And the high surface area of some nanoparticulate metal oxides serves to strongly chemisorb acidic gases or organic molecules for air purification.

- Information Storage: Nanoparticles are already used in audio and videotapes, which depend on magnetic and optical properties of fine particles. The control of magnetic coercivity and optical absorption will allow much denser storage media.

- Development of new sensors: When semiconductor nanoparticles are compressed to yield porous composites, they maintain their high surface areas. When these materials undergo a process of gas adsorption, the electrical conductivity changes are much stronger since the quantity of gas to be detected is much higher. Thus the sensitivity of sensors based on nanophase systems increases considerably.

- Smart magnetic fluids: ferrofluids are stable colloidal solutions of magnetic nanoparticles stabilized with surfactants, that can be used as vacuum or decontamination seals, and that have a great potential as cooling fluids or magnetically controlled heat conductors.

Perhaps one of the most promising areas on which Nanoscience is focused is Biomedicine, and the application of nanoparticles in health issues. Nanoparticles present very different and interesting mechanical, magnetic, structural and optical properties compared to those of the bulk material. Melting points, specific heats, coercive forces or surface areas are all size-dependent. The latter is especially remarkable, since the reactivity of a particle will strongly depend on the characteristics of its surface, and as size decreases the proportion of atoms on the surface per total of atoms rapidly increases. Besides, their small size allows their utilisation in in-vivo applications, i. e. inside the organism. Nanoparticles can be 
tagged with a biomolecule to be directed against the area of choice and there perform a specific action, such as the release of a chemotherapy agent, act as a contrast agent or selectively heat a specific area by applying an alternate magnetic field.

The research reported in this thesis has been oriented to the production and characterisation of novel magnetic particles encapsulated in inorganic coatings, and their application in Biomedicine. The work is divided into three major blocks: first, the description of the theoretical background, state-of the art and materials used throughout this work. In a second block, the synthesis and characterisation of four different types of magnetic nanoparticles. And finally, their actual use both in-vitro and in-vivo applications.

Chapter 1 is a short introduction to the main features concerning the physics at the scale of magnetic nanoparticles, and a brief description of some of the applications of nanoparticles in Biomedicine: both for diagnostics (MRI) and treatment of diseases (hyperthermia, drug delivery) or the magnetic separation of biomolecules and their use in biosensors.

In Chapter 2, materials and methods, the experimental procedures are described: the synthesis of the magnetic nanoparticles and the techniques used for their characterisation, the protocols used for the chemical functionalisation of the particles, as well as the material and methods used in the haematology studies and the in-vivo surgery animal model.

The morphological, physical and chemical characterisation of four different types of magnetic particles is presented in Chapter 3: iron nanoparticles encapsulated in carbon and silica produced by the discharge-arc method; magnetite nanoparticles embedded inside zeolite matrixes by mechanical activation, and magnetite crystallites encapsulated in amorphous silica obtained by a combination 
of the co-precipitation method and the sol-gel technique.

Chapter 4 deals with the functionalisation of silica and carbon surfaces of magnetic nanoparticles. The introduction of chemical groups on these surfaces is a necessary step to tag the particles with molecules which perform a specific action in the organism. The grafting of the specific antibody directed against the human chorionic gonadotropin (hCG) to the surface of the particles is described. The coating with serum proteins and the hydrophilic polymer poly(ethylene glycol) (PEG) has also been studied to avoid the action of the immune system inside a living organism, and therefore to allow a longer circulating time in the blood stream.

Chapter 5 comprises the results of the experiments performed in-vitro. The interaction of $\mathrm{Fe} @ \mathrm{C}$ particles with blood cells and platelets and the main haematological and rheological parameters have been studied to check the biocompatibility of circulating particles in blood. Besides, the uptake of $\mathrm{Fe} @ \mathrm{C}$ particles into dendritic cells (DCs) has been studied by electron microscopy and magnetic measurements allow localising and quantifying the amount of internalised magnetic particles. Also, the physical adsorption and release of the chemotherapy agent doxorubicin have been studied. The kinetics of adsorption and desorption of the drug has been assessed by vis-uv spectrophotometry.

A thorough description of all the experiments performed in-vivo is comprised in Chapter 6. A preclinical experimental animal model is described. Small permanent magnets were implanted inside deep solid organs of New Zealand rabbits. To achieve the targeted delivery of particles to the desired zone three different strategies were designed: intravenous injection of the particles through a marginal ear vein of the animal, intrarterial injection and direct inoculation of the particles suspension under echographic control. Histopathology studies assessed 
the distribution, behaviour and tolerance of nanoparticles in a living body after the insertion of the magnets.

Finally, a summary of the work and conclusions are comprised in the last chapter. References are all compiled in one last section, ordered alphabetically for an easier handling of Bibliography. A list of abbreviations is also included at the end of the manuscript. 



\section{Chapter 1}

\section{Introduction}

This first section is a short introduction to the main features concerning the physics at the scale of nanoparticles, and the changes in their physical and magnetic properties associated with size reduction. The main methods of preparation of nanomaterials are also described, as well as the importance of obtaining particles provided with a shell or encapsulation, in order to stabilise aqueous suspensions of these particles and render them biocompatible for any kind of in-vivo application.

This introduction chapter ends with the description of some of the main applications of nanoparticles in Biomedicine: both for diagnostics (MRI) and treatment of diseases (hyperthermia, drug delivery) or the magnetic separation of biomolecules and their use in biosensors, magnetix detoxification or magnetic cell sorting.

\subsection{Magnetism at the nanoscale}

Magnetism has its origin in the existence of intrinsic or induced atomic magnetic moments as a result of moving charges. From an atomic point of view, there are two electronic contributions: the orbital and the spin degrees of freedom of the electron, which are the source of intrinsic magnetic phenomena in materials [Sorensen2001]. 
From a magnetic point of view we can classify any kind of solid depending on its interaction with a magnetic field. The magnetism of a solid has its origin on the contributions of the electrons that constitute that solid. At macroscopic scale, the magnetic interaction between atoms and the crystalline structure of the solid give rise to the different magnetic response of materials. Non-magnetic materials have weak magnetic interactions, so that the thermal agitation at room temperature can make their magnetic moments flip over continuously, so that their average magnetic moment is very close to zero, displaying a linear response to an applied magnetic field: Paramagnetic materials have a net atomic or molecular magnetic moment and are weakly attracted towards a magnetic pole. For these materials magnetic susceptibility, $\kappa$, is a small and positive scalar: as $\mathrm{H}$ increases, $\mathrm{M}$ increases linearly. Some Iron salts, like chloride $\left(\mathrm{FeCl}_{3}\right)$ or sulphate $\left(\mathrm{FeSO}_{4}\right)$, Aluminium, Sodium or Oxygen gas and liquid display paramagnetic behaviour. Diamagnetic materials have neither net atomic nor molecular magnetic moment and are weakly repelled from a magnet. Their magnetic susceptibility is very small and negative, thus when we apply a magnetic field, $\mathrm{H}$, the induced moment will be small and opposed to the field. The variation of $\mathrm{M}$ with $\mathrm{H}$ is constant as well: as $\mathrm{H}$ increases, $\mathrm{M}$ decreases linearly. The vast majority of substances are diamagnetic, including water, wood, plastics, $\mathrm{SiO}_{2}, \mathrm{NaCl}, \mathrm{CaCO}_{3}$ and most of organic and biological materials.

Paramagnetic solids are not long range ordered and the thermal energy shakes each atom randomly, whereas diamagnetic solids have negligible field induced magnetic moments. In a ferromagnetic material, displacement of domain walls results in hysteresis cycles. On the contrary, for single domain particles in the superparamagnetic state the whole magnetic moment of each particle is shaken simultaneously in the same way as for a paramagnetic material (see Fig. 1.1, adapted from Goya2007). 


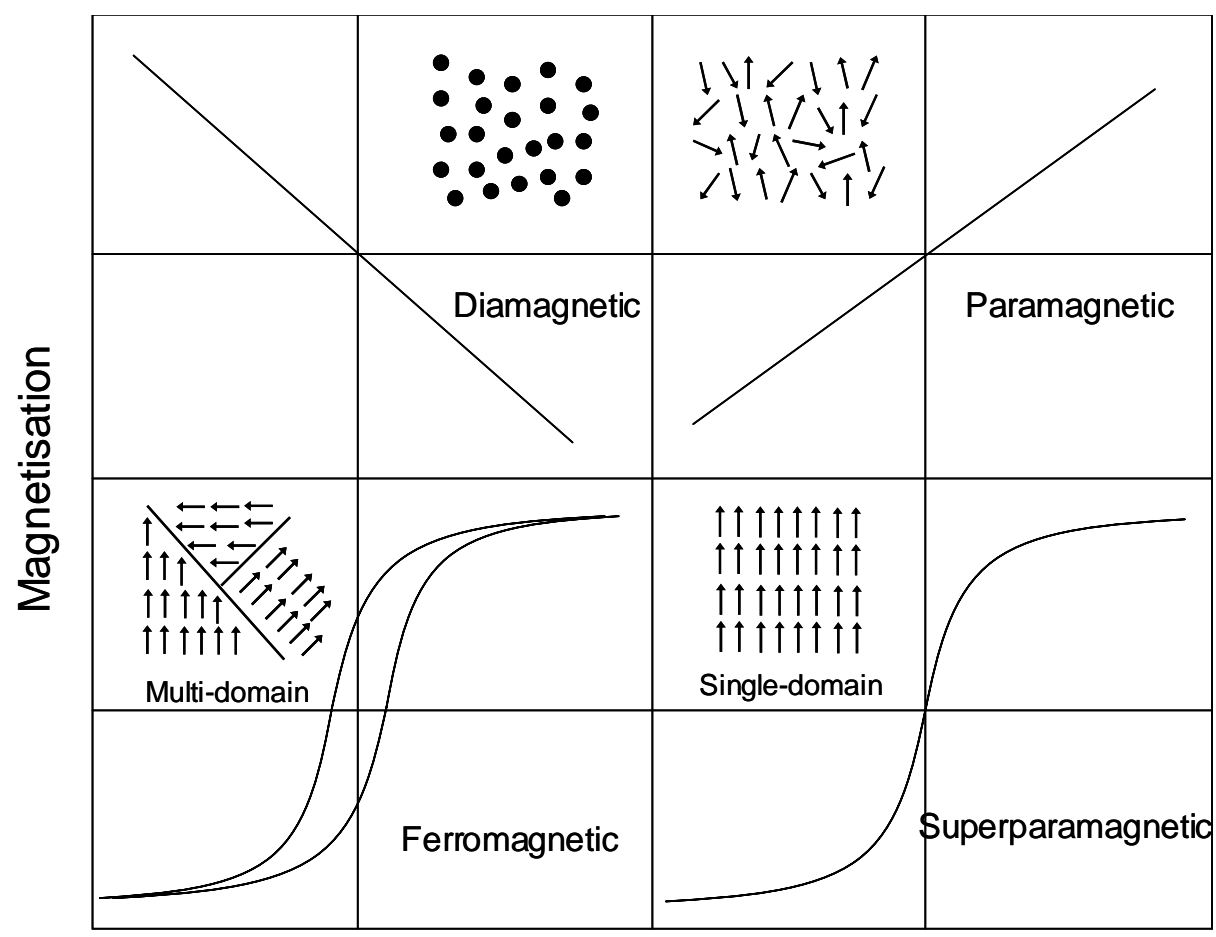

Field, $\mathrm{H}$

Figure 1.1 Variation of $\mathrm{M}$ vs. $\mathrm{H}$ for ferromagnetic, paramagnetic, diamagnetic and superparamagnetic materials.

If the magnetic interaction is of long range order due to the exchange interaction, the atoms within the solid can align the atomic magnetic moments parallel, such is the case of ferromagnets, or antiparallel, in the case of antiferromagnets. For the latter the antiparallel alignment can reduce the total moment to zero. When the antiferromagnetic interaction does not compensate exactly the magnetic moments a net ferromagnetic moment can be obtained, these kind of materials are called ferrimagnets. Ferro and ferrimagnetic materials are strongly attracted towards a magnet. In this case the magnetic susceptibility is large and positive. Iron, cobalt, nickel and some iron oxides, are archetypical ferromagnet whereas magnetite $\left(\mathrm{Fe}_{3} \mathrm{O}_{4}\right)$ or maghemite $\left(\gamma-\mathrm{Fe}_{2} \mathrm{O}_{3}\right)$ are some classical examples of ferrimagnets. Some 
magnetic phenomena associated with ferromagnetism are magnetic hysteresis, magnetic anisotropy, the existence of a transition temperature to paramagnetism, and the formation of magnetic domains.

\subsubsection{Hysteresis}

If we apply an increasing magnetic field on a ferromagnet and then decrease it, the magnetisation does not follow the initial magnetisation curve. This irreversibility is called hysteresis, and it occurs because the magnetic susceptibility of ferromagnetic materials is not a scalar. At high applied fields the magnetisation approaches the saturation magnetisation, $M_{s}$. If the field is decreased to zero, a remnant magnetisation remains, $\mathrm{M}_{\mathrm{r}}$. If we now apply a negative field, the magnetisation reaches zero at a field magnitude called the coercivity $H_{c}$. A symmetric behaviour of this hysteresis curve can be obtained as $\mathrm{H}$ is varied from positive to negative values (Figure 1.1). Permanent magnets as used in motors, generators or loudspeakers have large $\mathrm{M}_{\mathrm{r}}$ and $\mathrm{M}_{\mathrm{s}}$. Materials with $\mathrm{Hc} 100 \geq \mathrm{Oe}$ are called hard magnets, whereas soft magnetic materials are those with $\mathrm{H}_{\mathrm{c}} 10<\mathrm{Oe}$, commonly used for transformer cores and electronic circuits. In many biological applications, high values of $M_{s}$ and, specially, low values of $H_{c}$ are required, as we will see for the case of superparamagnetism, where coercivity is zero.

\subsubsection{Curie temperature}

The Curie point is the temperature above which a system undergoes a transition from ferromagnetic to paramagnetic. As the temperature is increased from below the Curie point, thermal fluctuations increasingly destroy the alignment of magnetic moments, until the net magnetisation becomes zero at and above the Curie point. Above that temperature, the material is purely paramagnetic. 


\subsubsection{Magnetic anisotropy}

The susceptibility of a material depends on the direction in which it is measured. This is called magnetic anisotropy. When this occurs, the total magnetisation of a ferromagnet, $\mathrm{M}_{\mathrm{s}}$, will prefer to align in a special direction called the easy axis. There are anisotropies due to the intrinsic properties of the material, as in the case of magnetocrystalline anisotropy, or they can be induced, for instance the case of shape anisotropy. Regarding the latter, it is easier to induce magnetisation along a long direction of a non-spherical piece of material than along the short direction, since the demagnetizing field is less in the long direction, as the induced poles at the surface are further from each other.

Ferromagnetic materials in absence of applied magnetic field have not net magnetisation because of the existence of magnetic domains. Their existence was proposed by Weiss in 1906. A macroscopic magnetic material breaks up into domains that align themselves to minimise the total free energy of the material. The energy of a magnetic field is proportional to the field squared and its volume extent. Thus the magnetostatic energy of a single domain of parallel spins can be decreased by breaking into smaller and oppositely aligned domains. The benefits of this decrease in energy would continue with the formation of smaller and smaller domains; however there is a competition between the magnetostatic energy and the exchange and anisotropy energy at the boundary (the domain wall) between oppositely aligned domains. This limits the process of restructuring to domains of a finite size.

As we have seen, the magnetostatic energy of a ferromagnet can be decreased by breaking up into domains. On the other hand, apparition of domains costs energy due to the formation of domain walls. Thus, there will be a critical domain size below which the cost of energy of domain walls formation exceeds the 
benefits from decreasing the magnetostatic energy. This critical size, or single domain size $D_{s}$, is where these two energies are equal. Typical values for $D_{s}$ range from 10 to $100 \mathrm{~nm}$, depending on the material (Table 1.2)

Table 1.2: Estimated single-domain size for spherical particles

\begin{tabular}{ll}
\hline Material & $\mathbf{D}_{\mathbf{s}}(\mathbf{n m})$ \\
\hline $\mathrm{Fe}$ & 14 \\
$\mathrm{Co}$ & 70 \\
$\mathrm{Ni}$ & 55 \\
$\mathrm{Fe}_{3} \mathrm{O}_{4}$ & 128 \\
$\gamma-\mathrm{Fe}_{2} \mathrm{O}_{3}$ & 166 \\
\hline
\end{tabular}

Ultrafine particles display unusual and very interesting properties compared to the bulk material. There is a limit of size below which the particle can not gain a favourable energy configuration restructuring into domains. As a consequence, they are single domain particles and present a magnetic behaviour known as superparamagnetism: this is characterised by a decoupling of the magnetisation of the particle from the lattice due to thermal energy which overcomes the anisotropy energy, which is proportional to the volume of the particle (KV).

With decreasing particle size, $\mathrm{KV}$ decreases until the thermal energy $\mathrm{kT}$ can cause fluctuations of the overall magnetic moment that take place within the particle. Thermal fluctuations can then affect the orientation of the magnetisation, $\mathrm{M}$, if they are large enough to overcome the magnetic anisotropy barrier. This change in magnetisation according to a temperature-dependent probability distribution resembles the behaviour of a paramagnetic material. The difference is that the amount of the individual magnetic moment, $\mu$, is several orders of magnitude larger because it is the addition of the moment of hundreds of thousands 
of atoms. For this phenomenon Néel coined the term superparamagnetism [Néel1949]. Two key features describe a superparamagnetic system, in analogy to the behaviour of paramagnetic materials: lack of hysteresis, and data of different temperatures superimpose onto a universal curve of $\mathrm{M}$ vs. H/T.

Superparamagnetism is a timescale-dependent phenomenon. The anisotropy energy KV represents an energy barrier to the total spin reorientation; hence the probability for jumping this barrier is proportional to the Boltzmann factor $\mathrm{e}^{-\mathrm{KV} / \mathrm{kT}}$. If we introduce the term $\tau_{0}$, which describes the timescale over which the magnetic moment of the particle $\left(\mu_{\mathrm{p}}=\mathrm{M}_{\mathrm{s}} \mathrm{V}\right)$ attempts to jump the anisotropy barrier, then the timescale for a change of orientation of the magnetic moment is:

$$
\tau=\tau_{0} \mathrm{e}^{-\mathrm{KV} / \mathrm{kT}}
$$

The attempt timescale, $\tau_{0}$, is about $10^{-9} \mathrm{~s}$. A typical experiment with a magnetometer takes 10 to $100 \mathrm{~s}$, so if total magnetisation $\mathrm{M}_{\mathrm{s}}$ reverses at times shorter than the experimental timescales, the system will appear superparamagnetic. Using $\tau=100 \mathrm{~s}$ and $\tau_{0}=10-9 \mathrm{~s}$, for the critical superparamagnetic volume $\mathrm{V}_{\mathrm{sp}}$ can be obtained:

$$
\mathrm{V}_{\mathrm{sp}}=25 \mathrm{kT} / \mathrm{K}
$$

Any particle with volume smaller than $\mathrm{V}_{\mathrm{sp}}$ will behave as a superparamagnet on the $100 \mathrm{~s}$ experimental timescale. Typical values of $\mathrm{V}_{\mathrm{sp}}$ are $16 \mathrm{~nm}$ for Iron and $7.6 \mathrm{~nm}$ for Cobalt. Also, eq. 1.2 can be rearranged:

$$
T_{B}=\frac{K V}{25 k}
$$


There is a critical temperature, called Blocking Temperature, $T_{B}$, below which the free movement of the individual magnetic moment is blocked by the anisotropy of the particle, and above which the thermal activation looses the moment so that the system appears superparamagnetic. The blocking temperature for any material can be measured with a magnetometer. The sample is cooled under zero applied field (zero-field-cooling, ZFC) to a temperature well below the expected $\mathrm{T}_{\mathrm{B}}$. Then a small field of $\sim 100$ Oe is applied. At this point, and presuming $\mathrm{T}<\mathrm{T}_{\mathrm{B}}$ the individual particles' moments are bound to the particles and point in random directions, so the induced magnetisation will be small. The system is slowly warmed, and as $\mathrm{T}$ approaches $\mathrm{T}_{\mathrm{B}}$, the thermal energy $\mathrm{kT}$ will start to loosen up the moments from the particles and the induced magnetisation will rise. At $T_{B}$ the moments are unblocked and free to align with the applied field to yield a large total magnetisation (see as an example Fig. 3.22 in page 99). Then a peak is observed for the curve $\mathrm{M}$ vs. T at the ZFC experiment. Once we have empirically measured $\mathrm{T}_{\mathrm{B}}$, from equation 1.3 values such as saturation magnetisation, anisotropy or size of the particles can be obtained.

Above the blocking temperature, the contribution from an ensemble of superparamagnetic particles to the magnetisation is described by the Langevin relation:

$$
M(H, T)=N \mu L\left(\frac{\mu H}{k_{B} T}\right)=N \mu\left(\operatorname{coth}\left(\frac{\mu H}{k_{B} T}\right)-\frac{k_{B} T}{\mu H}\right)
$$

Fitting this equation to the obtained experimental data the value of diameter of the particle can be obtained assuming spherical shape of the particle. 


\subsubsection{Coercivity of small particles}

Since there are no domain walls to move, the reversal magnetisation in singledomain particles is due to the spin rotation. As a consequence, the single-domain particles have a larger coercivity than multi-domain systems because it is harder to rotate the magnetisation than to move a domain wall. The largest coercivities occur exactly at the single-domain size. Below this, Hc decreases because the thermal activation is capable to pass the anisotropy barriers, being zero for superparamagnetic particles. (Figure 1.2) This is a very important property for a number of applications which demand particles with zero coercivity and zero remanence, that is, once the magnetic field is removed, particles do not interact with each other, and therefore will not remain aggregated.

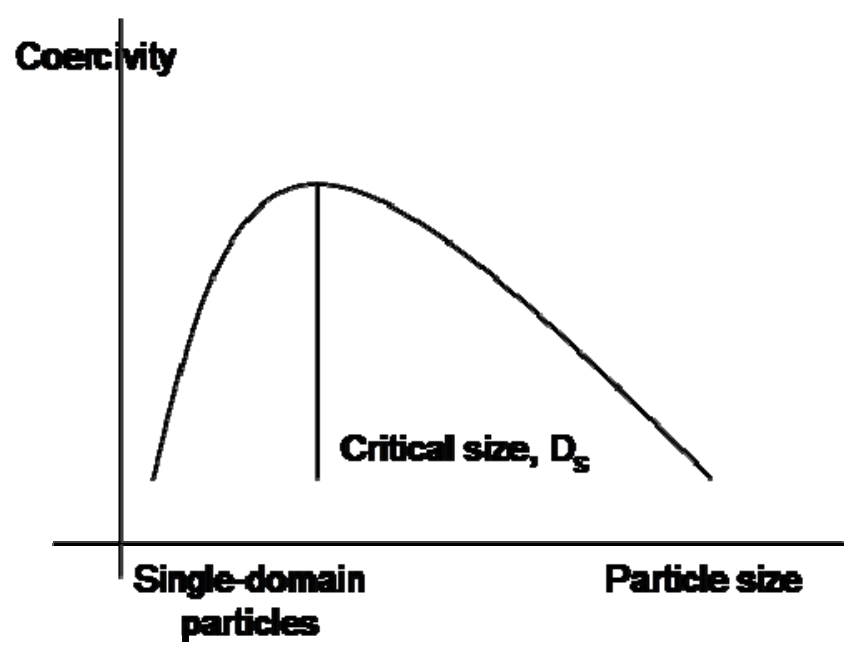

Figure 1.2 Particle size versus coercivity.

\subsection{Magnetic nanoparticles}

Inside the vast field of nanoparticulate systems, magnetic nanoparticles attract special attention due to their unique properties. These are nanostructures that contain a metal like iron, nickel or cobalt, or some of their oxides. Because of their 
response to an applied magnetic field they can be used as magnetic carriers in a number of biomedical applications, both in-vitro or in-vivo: by means of a magnetic gradient magnetic particles can be directed to a specific target and there release a specific drug or molecule (magnetic drug delivery); they can be used as contrast agents to enhance the image contrast in magnetic resonance imaging (MRI); they can also be heated selectively by applying an alternate magnetic field (magnetic thermal ablation or hyperthermia); or they can be functionalised with a molecule that specifically recognises an analyte and thus separate it from solution by means of a magnet (magnetic separation).

There are several requirements for the use of magnetic nanoparticles in biomedical applications: their size has to be small enough to avoid the action of gravitational forces that would make them precipitate in solution. Moreover, in the case of in-vivo applications (i.e. inside a living body) they have to be small enough to pass through the smallest capillaries in the body, and at the same time they have to be big enough to overcome the strength of blood flow. A balance between the two factors is then needed. Any material used in-vivo must be sterile and biocompatible as well, therefore the use of toxic metals like nickel, cobalt, neodymium or samarium is preferably avoided. Iron and iron oxides, especially Magnetite $\left(\mathrm{Fe}_{3} \mathrm{O}_{4}\right)$ and Maghemite $\left(\gamma-\mathrm{Fe}_{2} \mathrm{O}_{3}\right)$ are by far the most used nanosystems as magnetic carriers. Finally they must have high magnetic susceptibilities to respond rapidly and in an effective way to the action of a magnet. Bigger particles generally have higher saturation magnetisation and magnetic susceptibility than smaller ones, so a compromise is again required. The stability of magnetic particles suspensions is essential, and is not a trivial issue. It depends enormously on the synthesis process, hence an appropriate preparation and isolation of the materials is a must. 
1.2.1 General Methods of Synthesis: Top-Down vs. Bottom-Up Methods.

For the practical application of nanoparticles, it is not only a small size that matters. The conditions of synthesis need to be controlled so that resulting products have a uniform distribution in morphology, that is, the most similar size and shape as possible. Also an identical chemical composition and crystal structure are desirable so that the material presents reproducible reactivity and behaviour in its application. Finally, agglomeration of the particles needs to be avoided for any application; therefore the final purified products need to be rapidly dispersible in solution [Cao2004].

Many techniques have been developed, or are under development, so as to synthesize magnetic nanoparticles. Top-down approaches include mechanical milling, evaporation of bulk materials or lithography [Jewell1991]. All these methods are relatively quick, simple to handle and economic. Nanoparticles and nanocomposites can be thus obtained, along with a number of impurities and defects that need to be eliminated in a further treatment. The size of the obtained particles can be very small, but in general these are processes that are difficult to control in order to produce a specifically desired particle size and shape. For instance, High-energy ball milling (HEBM) can induce chemical reactions in powder mixtures, a process usually termed mechanochemistry. On the other hand, using lower energy ranges during the ball mill of solid/liquid mixtures results in homogeneous dispersion of fine particles without changes of the starting phases [Pankhurst2003].

On the other hand, bottom-up approaches are more utilised for the synthesis of small, monodisperse particles. Nanoparticles can be obtained by heterogeneous nucleation on solid substrates, or by homogeneous nucleation from liquid or vapour. These are the cases of co-precipitation and sol-gel techniques. In the first 
one the reaction of metallic salts in the presence of a base leads to the formation of nanostructured metal oxides. The latter is a wet chemical route for the synthesis of inorganic and hybrid materials, generally metal oxides and oxide-based composites. A sol-gel process consists of hydrolysis and condensation of precursors, which are usually metal alkoxides and inorganic or organic salts, dissolved in either aqueous or organic solvents. Sol-gel techniques have many advantages, including low processing temperature and molecular level homogeneity. An alternative to these methods is the confinement of chemical reactions, nucleation and growth processes inside small space, such as micelles [Fendler1987] or microemulsions [Fried1995], or inside droplets by evaporation of the precursors, as in the case of aerosol synthesis [Visca1978] or spray pyrolyis [Messing1993]. A good control of the particle size is achieved using these methods.

The process of growth from small molecules to bigger particles always involves several steps. First is nucleation, which takes place in a very short period of time, and it is achieved after supersaturation of the reagents that form the nuclei. The introduction of nanosized seeds is another possibility. Subsequent growth needs to be diffusion controlled, something achieved introducing a diffusion barrier, such as a polymer monolayer on the growth surface, using a low concentration of growth species or slowly generating growth species. In all of the cases, anyway, monodispersity is never completely fulfilled, so a further separation depending on size has to be done. Size-selective precipitation can be applied to separate larger particles from smaller ones. Magnetic separation is another possibility: by applying a magnetic field gradient larger aggregates can be removed from fine particles. A proper design of the geometry of the applied magnetic field is then essential to obtain an effective separation of the final materials. 


\subsubsection{Core-shell particles}

All the particles used during this research are Core-shell magnetic nanoparticles. They consist of a magnetic core encapsulated in an inorganic or polymeric coating. This coating renders the particles biocompatible, prevents them from oxidation in suspension, isolates them from each other, avoids the formation of magnetic aggregates, and serves as a support for the chemical bonding or physical adsorption of biomolecules on the surface of the particle.

The coating often plays a key role in the stabilisation of the particles in suspension. First of all it avoids leaching of the core, that is, the loss of magnetic material. Besides, the apparition of charges on the surface can stabilize liquid suspensions with a high saline concentration or slightly acid or alkaline $\mathrm{pH}$. For example, the isoelectrical point of silica is $\mathrm{pH}=2-3$, so at physiological $\mathrm{pH}=7.4$ the surface of silica will be negatively charged, inducing electrostatic repulsion between particles to help avoid aggregation. A low ionic force, i.e. a low concentration of salts in solution will also help to keep particles separated from each other to yield stable suspensions.

Depending on their size and their hidrophilicity or hydrophobicity, after systemic administration nanoparticles are most likely to be uptaken by the Mononuclear Phagocyte System (MPS) and cleared to the liver, spleen, and bone marrow. Different proteins (antibodies) of the blood serum (opsonins) bind to the surface of a nanoparticle (opsonization) and enhance the uptake of the particle by a phagocyte. Biodegradable (i.e., dextrane) and non-biodegradable organic coatings have been used to reduce the uptake by the macrophages of the RES, to avoid the lixiviation of a toxic core to the media (i.e. cobalt or gadolinium magnetic nanoparticles), and to provide a hydrophilic surface able to attach different functional groups or adsorb or link a specific drug. 
Inorganic coatings, like carbon or silica are also good candidates for attaining suspensions of particles that are suitable and long-term stable. Both materials are biocompatible and non-toxic. Also, amorphous silica is a heat-resisting material, with a low specific gravity, high surface area, and good mechanical strength. The small pore size of silica can give rise to a very selective interaction with the adsorbed molecules depending on their size, shape, and chemical characteristics.

Carbon and silica have hydroxyl $(\mathrm{OH})$ groups on the surface that can be substituted with more reactive chemical chains, that will be capable to bind covalently to the amine $\left(\mathrm{NH}_{2}\right)$ or carboxyl $(\mathrm{COOH})$ terminal groups of a protein, for example. The number of hydroxyl groups on the carbon surface is quite low in comparison to silica, and much less reactive. This fact makes carbon less attractive for chemical functionalisation but, on the other hand, it renders its surface highly hydrophobic, that way it easily adsorbs any organic molecule in solution. This is very important for the spontaneous adsorption and release of chemotherapy drugs in the case of drug delivery, and at the same time it makes carbonous particles a perfect target for the Rethiculoendothelial System (RES), since, due to the hydrophobic nature of their surface, they are more likely to undergo opsonization processes. So the objective is double: on one hand, to introduce on the particles some chemical groups that avoid the adsorption of opsonin, and therefore the action of the immunological defences of the body; and on the other hand the hydrophobic nature of carbon must be preserved so it can still be able to efficiently adsorb a drug.

Our strategy has been to first adsorb an amphyphylic molecule on the surface, PMAO, with a long hydrophobic chain on one side and carboxyl, hydrophilic groups on the other side, and then to covalently bind a Poly-ethyleneglycol chain (PEG) functionalised with amino groups at one of its ends. Once the particles have been functionalized with PEG, the presence of the polymer prevents the adsorption 
of big proteins of the blood and hence delays the recognition of the particle from the immune system. PEG is currently the most used molecule to prevent the action of the RES. The attachment of PEG to the nanoparticle surfaces provides a shielding "stealth" effect, delaying recognition and sequestration by the resident macrophages of the reticulo-endothelial system. Unfortunately, this 'immunostealthing' function is concurrent with the annihilation of biomolecular targeting capabilities, because the PEG molecules hide, in many cases, localizing antibodies conjugated on the nanoparticles surfaces which are used in antibody conjugated magnetic nanoparticles in MRI applications. In addition, the branching of the PEG could affect the diffusion of the drug in magnetic drug delivery applications, as well as the biodegradation of the core for SPIONs.

The presence of the polymeric coating is not an obstacle for small peptides or small molecules to be adsorbed on the surface of the particle. This is the case of chemotherapy drugs like doxorubicin which, despite the PEG barrier, is able to reach the surface of carbon and remain attached to it. Moreover, the presence of the coating serves to delay and control desorption of the drug, since now doxorubicin finds more obstacles to be released to the medium. 


\subsection{Biomedical Applications}

Biomedical applications of magnetic nanoparticles can be classified into therapeutic and diagnostic applications. Among the first ones the delivery of chemotherapy agents, magnetic hyperthermia and magnetic detoxification are the most extended applications. In diagnostics they are basically used as contrast agents in Magnetic Resonance Imaging (MRI) and magnetic separation by molecular recognition.

\subsubsection{Hyperthermia}

Hyperthermia, or thermal ablation, is the treatment of tumours by selective heating applying an alternating magnetic field (AC field). When temperature increases over a certain value, typically $42^{\circ} \mathrm{C}$, different mechanisms of cell damage occur in living tissues, basically death by necrosis or induction of death (apoptosis). The objective of hyperthermia is to increase the temperature only at a desired region, leaving other cells unaffected. Heating may be controlled by adjusting the field amplitude and the duration of field exposure, while the local temperature increase is measured online using optical fiber sensors introduced into the tumour tissue.

The control of temperature at that point is, therefore, essential, and is one of the major tasks in this research area. For this purpose a magnetic nanoparticles suspension is introduced into tumour tissue, achieving that way controlled and selective heating of tumoral cells. Energy is adsorbed and transformed into heat by the magnetic particles when a high frequency magnetic field is applied by the reversal of magnetisation inside a magnetic particle and by the rotation of the particle in a fluid suspension. The heating potential is strongly dependent on the particle size and shape, thus homogenous, uniform batches of particles are needed for a strict control on temperature. This technique is often used in combination with 
other treatments, like chemotherapy or irradiation. The goal of the scientific community is then to produce a magnetic nanoparticle that would, at the same time, serve for diagnosis and treatment of diseases, i.e., the particle should be able to load and release a drug, serve as an MRI contrast agent and also be heated selectively by the application of a magnetic field.

The concentration of magnetic particles in the target tissue and the increase of temperature that is required to damage tumoral cells determine the demand of specific heating power of magnetic particles for hyperthermia. This heating power is determined by the applied AC field. The general tendency is that as amplitude and frequency of the magnetic field increase, more heating power can be generated in particles. Because of biocompatibility restrictions, most studies have been performed with magnetic iron oxides magnetite and maghemite. The specific loss power (SLP) of a particle depends on the morphology and structure of the particle: for single-domain particles there is a rapid increase in SLP with increasing diameter of the particle, whereas for multidomain particles this trend is reversed. A narrow particle size distribution and a high magnetic anisotropy are also desirable to achieve a high SLP value.

The two main current limitations for the use of hyperthermia as a regular treatment are then the choice of the adequate particles, and the effective delivery of those particles to the desired area, to ensure a sufficiently high concentration of particles in the tumour tissue. This will depend on the degree of particles uptake inside tumour cells, either by endocytosis or by adsorption on cell membranes. For an effective delivery particles can be directly injected into the tumour or into blood vessels that supply the tumour; or they can be guided, either by the action of an external magnetic field, labelling the particles with antibodies that specifically recognise the presence of tumour cells. The targeting efficiency is still far from what would be desirable. The achieved concentrations of particles are so low until 
now that, for a useful heating treatment, considerable enhancement of the specific heating power of the magnetic particles would be needed. The problem is basically the same as for drug delivery: the functionalisation of the particles with tumourspecific antibodies is not an easy task, whereas the use of external magnets for magnetic guidance enriches the target region of the body but presents important geometrical limitations. Moreover, since magnetic attraction is proportional to the magnetic moment of the particle, superparamagnetic particles are not sufficiently targeted. On the other hand, the use of bigger, multi-domain particles presents the risk of aggregation and unintended embolization. Taking advantage of this fact, this method has been investigated for arterial embolization hyperthermia [Moroz2002].

Numerous studies of magnetic thermal ablation in-vitro have been reported, especially by the group of Andreas Jordan. Particles are introduced in cell cultures to test their biocompatibility and citotoxicity, and to determine the interaction of different types of particles with cells in aqueous suspensions. There are differences in the cellular uptake of magnetic nanoparticles by different cell types, as well for different coating of the particles [Jordan1999]. The toxicity of different coatings, including dextran, citrates, polyethyleneglycol or starch, has been studied by Häfeli and Pauer [Häfeli1999]. Suzuki et al [Suzuki1995] achieved a highly specific cellular uptake by coupling tumor-specific monoclonal antibodies onto the surface of magnetite particles coated by polyethyleneglycol. Significant increase of temperature in cell cultures have been reported [Jordan1999, Hiergeist1999], however we have to take into account that these systems are very well isolated, something that avoids undesired losses of temperature during the treatment and is not representative for the real situation inside a living body.

Hyperthermia has also been studied in-vivo [Shinkai2001, Moroz2002]. Mice, rabbits, dogs and pigs have been utilized for this purpose. Histological evaluation 
of the treated tissue proves that temperature elevation produces degradation of tumours. This treatment can be performed in animal experiments under tolerable systemic loads. There are some drawbacks, however, that have delayed the use of these techniques to the treatment of diseases in humans: first of all the equipment has been so far developed for animals so it has been traditionally too small. And, more important, there is no evidence of the curative value of magnetic particles hyperthermia yet, only a few cases of surviving lots with reduction or no further tumour growth after treatment [Jordan1997]. Best results have been reported by Yanase et al [Yanase1998] who identified $87.5 \%$ of rats with no tumour regrowth within three months. In spite of all these inconveniences first human trials have already started, and a Hyperthermia system for clinical application has been developed by Gneveckow et al [Gneveckow2004].

\subsubsection{Magnetic separation}

The principle of magnetic separation of biomolecules is based on the labelling of magnetic particles with adequate functional groups. These can specifically recognise a desired molecule, bind to it, and separate it from a suspension of other substances that may interfere in our analysis. Some of the main applications of Magnetic Separation techniques are cell sorting, magnetic detoxification and biomolecular recognition, on which the development of magnetic biosensors is based.

\subsubsection{Magnetic cell sorting}

One of the most widespread techniques related to magnetic separation is magnetic cell sorting. This has special interest in clinical analysis, since practically any type of cell can be isolated from complex cell mixtures: one example are commercially available kits of magnetic particles labelled with antibodies anti-CD3 that specifically recognise lymphocytes $\mathrm{B}$, that way we can separate lymphocytes $\mathrm{T}$ 
from B in a human blood sample. Currently, some of the most used applications of cell sorting could be: stem cell enrichment for graft engineering, T cell subsets in transplantation, Natural Killer cells for tumour therapy, stem cells in cardiac repair or enrichment of antigen-specific T cells for immune therapy [Ugelstad1995].

Magnetic Cell Separation, or MACS ${ }^{\circledR}$ technology, utilizes superparamagnetic nanoparticles for labelling the analites of interest and a separation column. First of all, individual cells of a cell suspension are magnetically labelled with magnetic particles, which have been previously conjugated to a monoclonal antibody or a specific ligand for a certain cell type. After that, the cell suspension is passed through the separation column that contains a ferromagnetic matrix, typically nickel wires or steel wool. A high magnetic gradient is applied by means of a permanent magnet, thus the particles and their conjugated analites will be retained in the wires, whereas non-labelled cells will pass through unnoticed. These cells can be easily eluted by rinsing with the appropriate buffer. Both separated fractions of cells could now be used for further use in flow cytometry, molecular biology, cell culture or clinical cellular therapy [Kantor1997]. MACS columns for research can process up to $2 \times 10^{10}$ cells, depending on the size of the column. This is clear advantage in comparison to Fluorescence-activated cell sorting (FACS), where cells are sorted one after the other, limiting the sorting speed to 5000 cells per second.

Before designing an experiment of cell sorting, it has to be taken into account that the optimal separation strategy depends on the amount of cells of interest in the sample, their phenotype compared with the other cells in the mixture, and restrictions of desired purity, yield or activation. There are two basic strategies for the separation of target cells with magnetic particles: positive selection and depletion. Positive selection is when the desired target cells are magnetically labelled and isolated directly as the positive cell fraction. This requires one or more 
cell surface markers which are specific for the target cells, and is specially recommended for the isolation of rare cells, such as hematopoietic stem cells from complex cell mixtures [Burt2003].

On the other hand, depletion means that the unwanted cells are those magnetically labelled and removed from the cell mixture, whereas the nonmagnetic fraction contains the cells of interest. This strategy is the most used since it can be applied to almost any kind of cells, as it is less aggressive on the functional status of the cell. Some typical examples of magnetic depletion are the selective removal of fibroblasts which often overgrow other cells from primary tissue cultures; the enrichment of nonhematopoietic tumour cells from blood and bone marrow of cancer patients by leukocyte depletion; and the depletion of $\mathrm{T}$ cells from allogeneic stem cell grafts [Barfield2003].

\subsubsection{Magnetic detoxification}

Magnetic detoxification of the blood can also be included among the applications of Magnetic Separation techniques. Removing toxins from the blood is still a challenge in medicine since most blood-borne toxins and biohazards cannot effectively be treated. Current methods for blood detoxification include hemodialysis and hemofiltration, plasmapheresis, extracorporeal immunoabsorption and direct injection of chelators or antibodies [Chen2007]. Even though these techniques have proved to be effective for the treatment of some diseases, there is still a need of improvement for the detection and treatment of certain intoxications, as in the case of biohazards or biochemical attacks. Magnetism increases significantly the sensitivity and performance of this method.

Magnetic detoxification systems are based on biocompatible magnetic nanospheres, which are functionalised with target-specific antitoxin receptors. They are then injected intravenously in the human blood stream. While they are in 
circulation they attach to toxins via the specific receptors on the surface. Toxinnanospheres systems are formed within a very short period of time, and via a venous or an arterial access the blood is passed through a high gradient magnetic separation device, where magnetically labelled toxins are retained. The detoxified blood is then returned into the body by extracorporeal circulation [Chen2007].

As in the case of any in-vivo application, the persistence of nanospheres in the blood stream over prolonged periods of time is basic for a more efficient removal of toxins in the blood. This persistence depends on the size of the particle, and the chemical surface of the spheres. Monodisperse particles, of homogenous morphologies are preferred to facilitate magnetic trapping and blood clearance. To avoid the removal from the blood of bare particles by protein adsorption and consequent macrophage capture nanospheres are usually coated with hydrophilic polyethylenglycol (PEG), derivatives. The copolymer poly(lactic-co-glycolic acid) is also a usual choice to avoid the action of the Immune system [Quellec1998, Xie2007].

\subsubsection{Separation and Magnetic Biosensors}

Conventional Immunochromatographic tests are based on the biomolecular recognition and capture of a certain colloidal coloured analyte, as it passes through a membrane on which the corresponding anti-analyte has been immobilised. These are very quick, simple and reliable diagnostic tools for many applications. However, along with their clear advantages, they have two main inconveniences: they are not able to quantify concentrations of analyte and their sensitivity is limited. Therefore, immunochromatographic tests are not adequate for certain applications, like the detection of infectious diseases. The use of magnetic nanoparticles bound to biorecognitive molecules, in substitution of conventional coloured colloids, like gold or dyed polymer spheres, permits to separate and to 
concentrate the analyte of interest, increasing the sensitivity of the test. Moreover, magnetically labelled targets generate a magnetic field than can be detected and measured directly with a magnetometer. The concentration of the molecule of interest can then be quantified. [Jianrong2004]. The quantification of the analyte can be performed either by inductive or magnetoresistive methods. The former consists of the detection and integration of the magnetic flux generated by magnetically labelled analytes. Quantum Design has a commercial prototype of a biosensor based on magnetoinductive detection. As an alternative, magnetoresistance-based sensors could lead to much higher sensitivities. The active component in a magnetoresistive sensor is a material that modifies its electric resistance when a magnetic field is applied. Currently, most used MR sensor are based on Giant Magnetoresistance phenomenon, also used in the hard drives of computers.

Magnetic separation has also been used in combination with optical sensing to perform magnetic enzyme linked immunosorbent assays (magnetic ELISA) [Kala1997]. These assays use fluorescent enzymes to optically determine the number of cells labelled by the assay enzymes. In a modification of this procedure the magnetic microspheres act as the surface for initial immobilisation of the target material and magnetic separation is used to increase the concentration of the material. [Pankhurst2003]. The mobility of the magnetic nanoparticles allows a shorter reaction time and a greater volume of reagent to be used than in standard immunoassays, where the antibody is bound to a plate. In a variation of this procedure, magnetic separation has been used to localise labelled cells at known locations for cell detection and counting via local scanning. Cells are labelled both magnetically and fluorescently and move through a magnetic field gradient towards a plate on which lines of ferromagnetic material have been lithographically etched. Cells align along these lines and the fluorescent tag is used for their optical detection. 


\subsubsection{Magnetic Resonance Imaging (MRI)}

Diagnosis of some diseases at their early stages remains one of the major tasks in modern medicine, since early diagnosis can be vital for the survival of the patient. Magnetic Resonance Imaging (MRI) is a powerful, non-invasive and nondestructive imaging tool, capable of providing 3D internal images of living organisms [Licha2005]. It provides high soft tissue contrast and high spatial resolution, and offers the possibility to display blood vessels, map brain functions or analyze cellular metabolisms.

Early diagnosis of small tumour cells can facilitate avoiding metastases and permits a more efficient treatment of the disease. However, the lack of sensitivity has hampered the application of MRI for a really efficient location of cancer at its early stage. The apparition of magnetic contrast agents has allowed clinical researchers and analysts to increase enormously the sensitivity levels with which tissues in a living body can be observed. The function of contrast agents in MRI is to enhance the image contrast between healthy and diseased tissue and indicate the status of organ functions or blood flow. Up to date most contrast agents have been paramagnetic systems, like Gadolinium chelates [Weinmann1984, Merbach2001]. The use of superparamagnetic iron oxide nanoparticles (SPIONS) is an alternative to conventional contrast agents: Combidex ${ }^{\circledR}$, Endorem ${ }^{\circledR}$ and Resovist ${ }^{\circledR}$ are already commercially available contrast agents. They are all core-shell nanoparticles composed of a maghemite core and a polymeric coating. These particles tend to accumulate in diseased organs due to differences in tissue composition and cell uptake processes [Kim2001]. Taking advantage of this fact, these particles are used for location and diagnosis of brain and heart infarcts, as well as liver lesions or tumours [Tartaj2003]. 
Despite the very small magnetic moment of a proton, the great number of protons that exist in biological tissue yields an effect than can be measured in the presence of large magnetic fields, and this effect is the base for MRI. If we have a large number of protons in the presence of an external magnetic moment $\mathrm{B}_{0}$, the net moment $\mathrm{m}$ precesses around $\mathrm{B}_{0}$ in the Larmor Frequency, $\omega_{0}$.

$$
\omega_{0}=\gamma \mathrm{B}_{0}
$$

Where $\gamma$ is the gyromagnetic ratio, for a proton $\gamma=2.67 \times 108 \mathrm{rad} \mathrm{s}^{-1} \mathrm{~T}^{-1}$.

A second external field is applied perpendicular to $\mathrm{B}_{0}$, oscillating at $\omega_{0}$. This second field is much weaker than $\mathrm{B}_{0}$, but it has the peculiarity of resonantly exciting the moment precession into the perpendicular plane. The oscillating field is then removed at time zero, and the in-plane and longitudinal moment amplitudes relax back to their initial values [Pankhurst2003]. The radio frequency transverse field is applied in a pulsed sequence, long enough in time to obtain a coherent response of the net magnetic moment of the protons to the MRI scanner. When the radio frequency is turned off the relaxation of the coherent response is measured in the scanner.

For $\mathrm{B}_{0}$ parallel to the $\mathrm{z}$ axis, relaxation signals are in the form:

$$
\begin{gathered}
\mathrm{m}_{\mathrm{z}}=\mathrm{m}\left(1-\mathrm{e}^{\mathrm{t} / \mathrm{T} 1}\right) \\
\mathrm{m}_{\mathrm{x}, \mathrm{y}}=\mathrm{m} \sin (\omega 0 \mathrm{t}+\varphi) \mathrm{e}^{\mathrm{t} / \mathrm{T} 2}
\end{gathered}
$$

$\varphi$ is a phase constant, $T_{1}$ is the longitudinal, also known as spin-lattice relaxation time, and $\mathrm{T}_{2}$ is the transverse, or spin-spin relaxation time. Longitudinal relaxation is associated with loss of energy as heat, from the system to the surrounding lattice. On the other hand, the loss of phase coherence in the precessing protons due to interaction between them and with other fluctuating 
moments in the media is the cause of the transverse relaxation. Often $T_{2}$ is replaced by the shorter relaxation time $\mathrm{T}_{2}{ }^{*}$, when local inhomogeneities in the applied longitudinal field can lead to extra dephasing phenomena:

$$
\frac{1}{T_{2}^{*}}=\frac{1}{T_{2}}+\gamma \frac{\Delta B_{0}}{2}
$$

Where $\Delta \mathrm{B}_{0}$ is the variation in the field caused by heterogeneity of the applied field itself, or due to local variations in the magnetic susceptibility of the system.

In living tissues the addition of contrast agents enhances contrast between adjacent anatomic structures. The magnetic moments of the added particles increase magnetic interactions during the collisions between molecules in a fluid, accelerating the relaxation of excited spins. Besides, dipolar interactions between superparamagnetic cores and surrounding protons from the fluid cause an increase in both longitudinal and transversal relaxation rates. New generation superparamagnetic agents are also known as $T_{2}$ contrast agents, since they produce a significant decrease in the transversal relaxation time, in opposition to paramagnetic particles like Gd-DTPA (Gadolinium diethylenetriaminepentaacetic acid) also called $T_{1}$ (longitudinal) agents. As a result, magnetic nanoparticles provoke local heterogeneity of the applied magnetic field in the vicinity of the targeted tissue and the signal is therefore enhanced [Richardson2005]. Recently, a work by Hartung et al described the labeling of macrophages using iron oxide particles of bacterial origin (magnetosomes). In an ex-vivo animal study the inflammation caused by induced peritonitis was identified by MRI using magnetosomes as contrast agents [Hartung2007].

Most significant improvements in sensitivity can be achieved when contrast agents are delivered to specific organs or tissues. For this purpose there are several 
solutions under development: the direct injection of contrast agents in the affected zone is the easiest and more obvious approach; it is however limited to regions of the body where access allows direct intervention. Another strategy is to utilize the size-dependent delivery taking into account the intrinsic porosity of the capillary vessels [Okuhata1999] and the specific characteristics of tumour vascularization. That way Combidex (commercial superparamagnetic iron oxide) nanoparticles have been delivered by accumulation in noncancerous lymph tissue [Shelley2006]. Promising results have been achieved in the improvement of sensitivity of detection and delineation of pathological structures, such as primary and metastatic brain tumours, inflammation or ischemia [Roberts2000].

One of the most encouraging alternatives for the targeted delivery of these agents is to bind monoclonal antibodies to the surface of magnetic nanoparticles that specifically recognise certain receptors of the damaged cells at the desired organ. After the work by Suzuki et al [Suzuki1996] many research groups have focused their interest in the design and development of antibody-conjugated magnetic nanoparticles. When cancer spreads, there is an overexpression of certain factors at the tumoral cells. In order to reach selectively the target malignant cells antibodies anti-VEGF and anti-VCAM antibodies have been linked to magnetic polymeric particles. In the same direction, Huh et al synthesised magnetic nanocrystals conjugated with herceptin, a cancer-targeting antibody [Huh2005]. Alternatively, the use of other biomolecules for specific reception at diseased tissues has been reported: Proteins such as transferring [Högemann2000], peptides like the membrane translocating tat peptide of the HIV tat peptide protein [Josephson1999] and oligonucleotides [Josephson2001] have been attached to iron oxide nanoparticles to obtain specifically deliverable MR imaging agents [Högemann2002, Tartaj2003]. 


\subsubsection{Drug delivery}

The targeted delivery of drugs is an elegant and non-invasive strategy to treat certain diseases. Magnetic drug delivery utilizes magnetic carriers that can be loaded with the therapy agent and directed towards a desired zone, like tumour cells or blood clots, by means of a magnetic field gradient. The drugs are adsorbed on the surface or embedded inside these nanosystems, and ideally released only at the target organ. Drug release can take place by simple diffusion or proceed through mechanisms that require enzymatic activity, magnetic triggering or changes in physiological conditions such as $\mathrm{pH}$, osmolality or temperature [Alexiou2000].

Two aspects have to be taken into account when tackling the problem of local drug delivery: the choice of the adequate magnetic carriers, and how to direct the particles to the target.

\subsubsection{Magnetic Carriers for local drug delivery}

Because of their biocompatibility and magnetic properties iron oxide nanoparticles are the preferred material for most biomedical applications. They consist of a magnetite $\left(\mathrm{Fe}_{3} \mathrm{O}_{4}\right)$ or maghemite $\left(\gamma-\mathrm{Fe}_{2} \mathrm{O}_{3}\right)$ core encapsulated in an organic [Massart1987, Bautista2005] or inorganic matrix [FernándezPacheco2006]. An alternative is the use of magnetic nanocapsules, where the drugs are confined inside a cavity surrounded by an inorganic membrane [Arruebo2006a] or a bilipid layer as in the case of magnetic liposomes [Gonzales2005].

The use of magnetic micro and nanoparticles for drug delivery has evolved since the 1970's [Arruebo2007a]. First results refer only to the use of micron size particles. Zimmerman and Pilwat [Zimmerman1976] used magnetic erythrocytes for the delivery of cytotoxic drugs. Widder et al [Widder1978] described the 
targeting of magnetic albumin microshperes encapsulating a chemotherapy agent doxorubicin in animal models. In the 1980s several authors developed this strategy to deliver different drugs using magnetic microcapsules and microspheres [Kato1984, Widder1981, Gupta1989, Morimoto1980]. In the 1990s Häfeli et al prepared biodegradable poly(lactic acid) microspheres that incorporated magnetite and the $\beta$-emitter ${ }^{90} \mathrm{Y}$ for targeted radiotherapy, and successfully applied them to subcutaneous tumors [Häfeli1995].

Magnetic nanoparticles were used for the first time in animal models by Lübbe et al [Lübbe1996a]. The first phase I clinical trial was carried out by the same group in patients with advanced and unsuccessfully pretreated cancers using magnetic nanoparticles loaded with epirubicin [Lübbe1996b]. That first trial faced the main problem that researchers try to overcome to achieve an efficient delivery of magnetic carriers: most of the particles ended up in the liver, trapped by macrophages.

The case of the company FeRx Inc. is also significant of the difficulty to succeed in this field. Founded in 1997, they produced Iron particles encapsulated in activated carbon by mechanical milling, and loaded them with doxorubicin. A phase II clinical study in patients with primary liver cancer was conducted, but the trial was unsuccessful, and the company finally closed down. Chemicell $\mathrm{GmbH}$ currently commercialises TargetNAG-doxorubicin nanoparticles involving a multidomain magnetite core and a cross-linked starch matrix with terminal cations that can be reversibly exchanged by the positively charged doxorubicin. These particles have a hydrodynamic diameter of $50 \mathrm{~nm}$ and are coated with $3 \mathrm{mg} / \mathrm{ml}$ doxorubicin [Steinfeld2006]. These nanoparticles loaded with mitoxantrone have already been used in animal models with successful results [Wiekhorst2006]. Chemicell also commercialises FluidMAG ${ }^{\circledR}$ for drug delivery applications. Magnetic nanoparticle hydrogel (MagNaGel $\left.{ }^{\circledR}\right)$ from Alnis Biosciences Inc. is a 
material comprising chemotherapeutic agents, Fe oxide colloids, and targeting ligands [Sunderland2006].

\subsubsection{Toxicity}

Before the use of any magnetic carriers for any in-vivo application it is necessary to analyze its toxicity (acute, subacute and chronic toxicity, teratogenity and mutagenicity) in cellular and animal models, haematocompatibility, biodegradation (if possible), immunogenicity and pharmacokinetics: body distribution, metabolism, bioavailability, elimination, and organ specific toxicity before starting with the preclinical testing. Toxicity depends predominantly on the size, composition and the coating [Macaroff2006]. Tailoring the magnetic nanoparticles surface will be the main point to minimize any toxicological effect [Park2006]. In general it is accepted that larger magnetic particles show higher cytotoxicity than smaller ones [Yin2005]. It is well documented that the large surface area to volume ratio of all nanosized particles can potentially lead to unfavourable biological responses if they are inhaled and subsequently absorbed via the lung or swallowed and then absorbed across the gastrointestinal tract [Duncan2005b]. And not only acute toxicity but also the toxicity of degradation products, stimulation of cells with subsequent release of inflammatory mediators, and toxic effects through the particulate system have to be seriously considered [Neuberger2005].

\subsubsection{Administration and delivery}

There are three parameters that determine the behaviour of magnetic nanoparticles inside the organism: the surface chemistry, size, including magnetic core, hydrodynamic volume, and size distribution, and the magnetic properties of the nanoparticles, especially their magnetic moment, remanence, and coercivity. The surface chemistry is very important to hinder the action of the 
reticuloendothelial system (RES) and avoiding a fast removal. The nanoparticles usually need to be coated with a neutral and hydrophilic compound (i.e., polyethylene-glycol, polysaccharides, dysopsonins) increasing the circulatory clearance half-time from minutes to hours or days. Another possibility is to reduce the particle size; however, despite all efforts complete evasion of the RES has not yet been possible and additional migration to unwanted areas in the body may cause toxicological problems.

The fate and toxicity of the magnetic nanoparticles mainly depends on the size, dose and via of administration into the body. The latter can be divided into oral or parenteral, including intravenous delivery, pulmonary delivery, transdermal delivery, ocular delivery, and even as coatings in scaffolds. Three most common ways of administration are intravenous, oral and direct injection in the tumour.

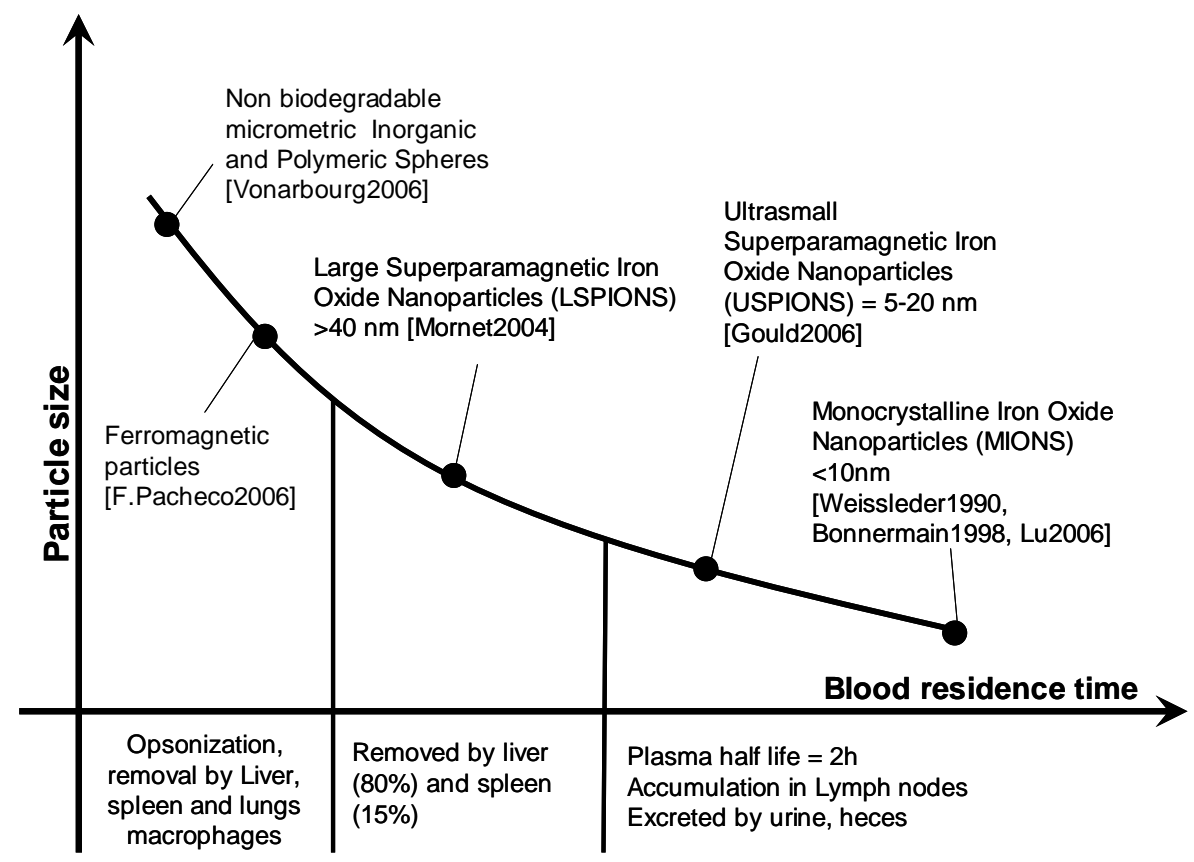

Figure 1.3 Evolution of blood residence time with particle size [Arruebo2007a]. 


\subsubsection{Intravenous administration}

For parenteral application it is essential that the carrier is non-toxic and nonimmunogenic, and it needs to be small enough to avoid embolization in the capillary vessels when intravenous application is used. Once a nanoparticle enters the bloodstream, the opsonization process activates the response of the reticuleendothelial system (RES). The circulating mononuclear phagocytes (monocytes) clear the nanoparticle to the liver, spleen, and bone marrow principally where resident cells (i.e, Kupffer cells in the liver) from the same system capture the nanoparticles for their degradation if this is possible. Some of the nanoparticles present in the lysosomal vesicles of Kupffer cells (depending on the biodegradability and size) are incorporated in the bile and through the digestive to the faeces. Some of them after the action of the liver will be filtered by the kidneys and incorporated in the urine. In general, the smaller nanoparticles are subjected to rapid renal elimination whilst the larger ones show liver, spleen, and bone marrow uptake. Large particles will be only removed by cells capable of phagocytosis (i.e, by macrophages, dendritic cells), small particles will be removed by cells capable of endocytosis (i.e., by lymphocytes T), and if the magnetic nanoparticles are biodegradable the decomposition products can be up-taken by all the cells by means of pinocytosis.

According to Neuberger et al. [Neuberger2005] magnetic particles smaller than $4 \mu \mathrm{m}$ are taken up through cells of the reticuloendothelial system, mainly in the liver (60-90\%) and spleen (3-10\%). While it is more likely that small particles up to $100 \mathrm{~nm}$ will be phagocytosed through liver cells (openings in the endothelium of liver sinusoids are between 100 and $150 \mathrm{~nm}$ ), there is a tendency for particles larger than $200 \mathrm{~nm}$ to be filtered by the venous sinuses of the spleen. If particles between 30 and $100 \mathrm{~nm}$ are intravenously applied, the macrophages of the liver (Kupffer cells) eliminate the larger particles faster from the bloodstream 
compared to the smaller sizes. Thus, the larger the particles are, the shorter is their plasma half-life-period. This clearance of particles by the kupffer cells can be, on the other hand, useful for the treatment of diseases produced in the liver, such as liver cancer or leishmania [Chellata2005].

\subsubsection{Oral administration}

The use of magnetic nanoparticles coated with an organic shell for using them as oral drug delivery vectors has been described [Cheng2006b], but most of the literature is referred to magnetic nanoparticles used as contrast agents for the gastrointestinal tract in MRI. The main problem that should be addressed is that oral delivery of peptides and proteins is hampered by various factors including their gastrointestinal degradation, low absorption, and first-pass metabolism by the liver. In addition, drugs delivered orally produce a significant initial increase in drug concentration followed by a peak above the therapeutic range. In 2003 the work of Feng et al. [Feng2003] describes the fate of chemotherapyl nanoparticles for oral delivery. Hence particles smaller than $5000 \mathrm{~nm}$ can be taken up via the lymphatics, particles smaller than $500 \mathrm{~nm}$ can cross the membrane of epithelial cells through endocytosis, and particles below $50 \mathrm{~nm}$ can achieve paracellular passage between intestinal epithelial cells.

\subsubsection{Subcutaneous or direct injection into the tumour}

Unlike water-soluble molecules, which are rapidly absorbed through the blood capillary wall and pass into circulation, small particles injected locally infiltrate into the interstitial space around the injection site and are gradually absorbed by the lymphatic capillaries into the lymphatic system [Brigger2002]. For that reason, subcutaneously or locally injected (in the peritumoral region) nanoparticles can be used for lymphatic targeting, i.e. as a tool for chemotherapy against lymphatic 
tumours or metastases. This route is rarely used in clinical practice, as it is not useful for targeting metastatic tumours.

\subsubsection{External magnets and magnetic implants}

The second issue that has to be dealt with is the effective delivery of magnetic carriers to the target. The classical approach has been the use of an external source of magnetism, like an electromagnet, applied on the desired area to generate a magnetic field gradient and thus localise and concentrate magnetic particles. However, the use of external magnets presents serious limitations. The homogeneity of the magnetic field on the target zone generates very weak field gradients, unable to concentrate the carriers in an effective way. Because of the weakness of magnetic forces, which have to overcome hydrodynamic forces in the blood stream, the administration method is limited to an artery close to the tumour, it can only be applied to the treatment of superficial organs and, moreover, well defined magnetic field geometries are a must, depending on the tumour allocation, to perform an efficient magnetic drug delivery [Shinkai2002, Lübbe2001, Alexiou2005].

To avoid these inconveniences, the insertion of small magnetic implants directly into the affected zone, or the combination of both external and internal sources of magnetism, appear as a promising alternative. A small stent or a magnetic wire is inserted near the diseased area, and an external source of magnetism is applied, magnetizing the implant. An internal local magnetic field is thus created, rather more effective than those produced by external magnets.

Several strategies have been described to achieve an effective targeted accumulation of the drug carriers. Yellen et al. suggested the use of two independent magnetic sources. A small size magnetic mesh, consisting of a $3 \times 3$ array of magnetic wires, in combination with long range externally applied fields 
were used to magnetize and localize the carriers in an in-vitro experiment [Yellen2005].

Avilés and coworkers designed a coiled ferromagnetic wire stent in order to simulate an intravascular stent in-vitro. They used magnetite nanoparticles embedded in poly(styrene/divinylbenzene) matrixes as carriers, and an external permanent magnet to magnetize the wire stent. Changes at the capture efficiency by varying the applied magnetic field, the particle concentration and the fluid velocity were studied [Avilés2007].

The research presented in chapter 6 of this work: the implantation of small permanent magnets by minimally invasive surgery is an alternative to these procedures. First in-vivo experiments of localisation of magnetic nanoparticles with implanted permanent magnets have already been performed in rabbits [FernándezPacheco2007].

\subsubsection{Non-magnetic delivery systems}

As an alternative to magnetic carriers, different organic materials such as polymeric nanoparticles, liposomes, and micelles have been investigated as drugdelivery nanovectors which target a specific area in the body by means of passive targeting or with a recognition moiety (i.e., antibody). However, search for different alternatives continues in view of a variety of still unsolved problems of these systems, such as their limited chemical and mechanic stability, swelling, susceptibility to microbiological attack and the inadequate control over the drugrelease rate [Arruebo2006a].

One of the main disadvantages of functional polymers for biomedical applications is their high polydispersity. Their synthesis produces either very small or very large molecules (irregular branching) which could exhibit non-uniform 
pharmacological properties. That disadvantage was solved by the use of dendrimers which show monodisperse character and globular architecture. This monodispersity results from the stepwise synthesis used in their preparation and the ability to purify dendrons and dendrimers at each step of growth [Fréchet2005a]. Dendrimers, as other nanoparticles used for biomedical applications, need to be biocompatible, with high plasma-residence time, and water soluble [Fréchet2005b].

It is still a challenge to prepare dendritic polymers that circulate in the blood long enough to accumulate at target sites, but that can be also eliminated from the body at a reasonable rate to avoid long-term build-up. In addition, the tissue localization of dendritic polymers is still difficult to predict in advance and more studies are required to determine the effect of peripheral dendritic groups on these properties. To visualise the dendrimers it is necessary to tag them with a specific moiety (i.e, fluorophore, metal).

Passive targeting using drug conjugated dendrimers and dendritic polymers is the most extensively studied targeted delivery using the increased permeability of tumour vasculature to molecules and its limited lymphatic drainage. Therapies based on active targeting such as antibody conjugated dendrimers have been developed for boron neutron capture therapy; however a full biological evaluation of these systems has not been reported. Antibody conjugated drug-loaded dendrimers show promising alternatives, although antibodies have exquisite potential for selective targeting but, even as humanized proteins, they can be immunogenic [Duncan2005a]. 


\section{Chapter 2}

\section{Materials and Methods}

This chapter is a description of the experimental procedures that have been utilised throughout this work: the synthesis of the magnetic nanoparticles and the techniques used for their characterization, the protocols used for the chemical functionalisation of the particles, as well as the material and methods used in the haematology studies and the in-vivo surgery animal model.

\subsection{Characterization techniques}

In order to choose the most adequate particles for each application, we need to know the morphology, physical properties, chemical reactivity and biological behaviour of the produced materials. The properties of nanoparticles rely especially on their size, shape, chemical nature of the core and reactivity of the functional groups on the surface. Several spectroscopy techniques have been used to study the chemical composition of the obtained compounds. X-Ray and Selected Area Diffractions allow us to determine the crystalline structure of the metallic cores of the particles. Electron Microscopy gives us information on the morphology and heterogeneity of the samples. Finally, magnetic measurements are required to determine the magnetic properties of the materials at different temperatures.

\subsubsection{Magnetometry}

The magnetic properties of the compounds were studied with a superconducting quantum interference device (SQUID MPMS-5S, Quantum 
Design). DC Measurements were performed up to $50 \mathrm{KOe}$ (5 Tesla). The temperature range was $5 \mathrm{~K}$ to $300 \mathrm{~K}$. A SQUID is a superconducting ring immersed in liquid helium with two identical Josephson junctions. Samples can be measured either as solids or in aqueous suspensions. The latter have to be frozen for measurement, since above the melting point of water particles can rotate inside the liquid, leading to experimental errors.

Measurements of magnetisation Vs applied field at a given temperature provide us information about the response of our particles to a generated magnetic gradient. Superparamagnetism, or zero value for coercivity and remanence, may be preferred for certain applications that require the particles to remain not aggregated after removal of the applied magnetic field. Measurement of magnetisation with increasing temperature allows us to estimate the size of the magnetic core of the particle and the size polydispersity of a sample, as well as calculate the blocking temperature of our sample, i. e. the temperature above which a material behaves as superparamagnetic.

\subsubsection{Scanning Electron Microscopy (SEM)}

The morphology of the particles can be studied at a first approach by scanning electron microscopy. SEM yields images of the particles with a resolution from a few milimeters to less than $50 \mathrm{~nm}$. In a SEM microscope, the surface of the sample is irradiated with high energy electrons. A set of magnetic lenses moves the focused beam back and forth across the specimen. As the electron beam hits each spot on the sample, both electrons and photons are emitted by the specimen surface, and their intensity is used to form the SEM image when all the spots are convoluted. The signals most commonly used are the Secondary Electrons, the Backscattered Electrons and X-rays. An electron detector is used to convert the 
radiation of interest into an electrical signal for manipulation and display by signal processing electronics.

Using the secondary electron detector a clear and focused topographical image of the sample is obtained. The backscatter electron detector produces an image that is useful when determining the make-up of the sample. Each element in the sample appears as a different shade, from almost white to black. Secondary electrons are specimen electrons that obtain energy by inelastic collisions with beam electrons. They are defined as electrons emitted from the specimen with energy less than $50 \mathrm{eV}$. On the other hand, backscattered electrons are the primary incoming beam electrons that experience an elastic backscattering with sample electrons. The number of backscattered electrons will produce a contrast depending on the mean atomic number of the illuminated spot. That way, 2D phase analysis can be performed comparing the different contrasts.

Finally, X-rays are emitted when deep levels electrons are pulled out from the sample atoms. The energy of the emitted photon will be characteristic of the electronic configuration of each atom. The analysis of the X-ray fluorescence spectra gives the chemical composition with the spatial resolution of the beam spot. This technique is known as Energy Dispersive X-ray microanalysis (EDAX).

\subsubsection{Transmission Electron Microscopy (TEM)}

The detailed structural and morphological characterization of our samples was carried out by means of transmission electron microscopy (TEM) and the power spectra obtained by using Fast Fourier Transform (FFT) algorithms on the highresolution TEM (HRTEM) micrographs. In order to obtain the high-resolution TEM (HRTEM) results we used a field emission gun microscope Jeol 2010F, at the Serveis Cientificotècnics of the Universitat de Barcelona, which works at $200 \mathrm{kV}$ and has a point-to-point resolution of $0.19 \mathrm{~nm}$. Electron energy loss (EELS) spectra 
were obtained in a Gatan Image Filter (GIF 2000) coupled to the Jeol 2010F microscope. Spectra were obtained with an energy resolution of $1.2 \mathrm{eV}$.

In a TEM microscope a beam of electrons is transmitted through a specimen, and then an image is formed, magnified and directed to appear on a layer of photographic film, or detected by a sensor such as a CCD camera. The difference is the energy of the incident electrons, which is higher than in the case of TEM in an order of magnitude (20-30kV for SEM compared to $200-300 \mathrm{kV}$ of the TEM). These electrons can now penetrate in the sample and give us information of the morphology, size or crystalline structure. Electrons are emitted from a field emission gun and focused by means of magnetic lenses, whose magnetic fields deviate the trajectories. A final lens sets the sample either on the image or on the focal plane. In the first case a magnified image of the sample is obtained at the CCD camera. On the contrary, when the sample is focused on the focal plane an image of the reciprocal space is taken, then electron diffraction can be performed. A diaphragm placed at the image plane can select a certain $300 \mathrm{kV}$ of the TEM). These electrons can now penetrate in the sample and give us information of the morphology, size or crystalline structure. Electrons are emitted from a field emission gun and focused by means of magnetic lenses, whose magnetic fields deviate the trajectories. A final lens sets the sample either on the image area of the sample for diffraction, a technique known as Selected Area Diffraction Pattern (SADP), a tool that allows to characterise crystalline structures at a selected point of the sample [Williams1996].

In Electron Filtered Transmission Electron Microscopy (EFTEM) only electrons of particular kinetic energies are used to form the image or diffraction pattern. The technique can be used to aid chemical analysis of the sample to complement electron crystallography. To obtain these "coloured maps" of elements we must first perform Electron Energy Loss Spectroscopy (EELS) analysis. In 
EELS the material is exposed to a beam of electrons with a known, narrow range of kinetic energies, then some of the electrons undergo inelastic scattering, which means that they lose energy and have their paths slightly and randomly deflected. The amount of energy loss can be measured via an electron spectrometer and interpreted in terms of what caused the energy loss. Inelastic interactions include phonon excitations, inter and intra band transitions, plasmon excitations, inner shell ionisations, and Cherenkov radiation. The inner shell ionizations are particularly useful for detecting the elemental components of a material [Brydson2006].

\subsubsection{Fourier Transformed Infrared Spectroscopy (FTIR)}

FTIR data were obtained using a Mattson Research Series spectrometer in air at room temperature. The solids were homogeneously dispersed in $\mathrm{KBr}(2 \%$ wt. approximately) and pressed into discs. The spectra were recorded with a resolution of $4 \mathrm{~cm}^{-1}$ with 80 scans per spectrum.

Chemical bonds have specific frequencies at which they vibrate corresponding to energy levels. The resonant frequencies or vibrational frequencies are determined by the shape of the molecular potential energy surfaces, the masses of the atoms and, eventually by the associated vibronic coupling. Resonant frequencies can be in a first approach related to the strength of the bond, and the mass of the atoms at either end of it. Thus, the frequency of the vibrations can be associated with a particular bond type. In order to measure a sample, a beam of infrared light is passed through the sample, and the amount of energy absorbed at each wavelength is recorded. This may be done by scanning through the spectrum with a monochromatic beam, which changes in wavelength over time, or by using a Fourier transform instrument to measure all wavelengths at once. From this, a transmittance or absorbance spectrum may be plotted, which shows at which 
wavelengths the sample absorbs the IR, and allows an interpretation of which bonds are present [Lau1999].

In our case IR spectroscopy allows us to identify the effective coating of the particles, through the study of the interaction between the metallic core and the inorganic matrix that encapsulates the particles. By means of IR we can also track the introduction of functional groups covalently bond to the surface of the particles: Carboxyl and amine groups have characteristic transmittance peaks so they can be easily identified in the final products by comparison with the initial reactants.

\subsubsection{Ultra Violet -Visible Spectroscopy (UV-VIS)}

UV-VIS spectroscopy is routinely used in the quantitative determination of solutions of transition metal ions and highly conjugated organic compounds. The Beer-Lambert law states that the absorbance of a solution is directly proportional to the solution's concentration. Thus UV/VIS spectroscopy can be used to determine the concentration of a solution. It is necessary to know how quickly the absorbance changes with concentration. This can be taken from references (tables of molar extinction coefficients), or more accurately, determined from a calibration curve.

A UV-VIS spectrophotometer measures the intensity of light passing through a sample $(I)$, and compares it to the intensity of light before it passes through the sample $\left(I_{o}\right)$. The ratio $I / I_{o}$ is called the transmittance, and is usually expressed as a percentage $(\% \mathrm{~T})$. The absorbance, $A$, is based on the transmittance:

$$
A=-\log (\% T)
$$

The basic parts of a spectrophotometer are a light source (often an incandescent bulb for the visible wavelengths, or a deuterium arc lamp in the ultraviolet), a holder for the sample, a diffraction grating or monochromator to 
separate the different wavelengths of light, and a detector. In our spectrophotometer the detector is a photodiode. Photodiodes are used with monochromators, which filter the light so that only light of a single wavelength reaches the detector.

The adsorption and release of proteins or drugs from the surface of the particles can be studied by UV-VIS Spectroscopy with an indirect approach: the particles are sedimented with a magnet or centrifuged and the supernatant is measured. The difference between the starting concentration of the organic molecule that binds to the particle and the remaining concentration in solution is the actual concentration of the molecule adsorbed on the particle.

\subsubsection{X-Ray diffraction (XRD)}

Powder X-ray diffraction (XRD) was performed on a Rigaku/Max diffractometer using filtered $\mathrm{Cu} \mathrm{K} \alpha$ radiation at the Servicio Nacional de EXAFS at the ICMA. XRD allows the identification and characterisation of crystalline solids. In the case of magnetic oxides, each of which produces a distinctive diffraction pattern. Both the positions, corresponding to lattice spacings, and the relative intensity of the lines are indicative of a particular phase and material, providing a "fingerprint" for comparison. Powder XRD can also be used to determine the partial crystallinity or amorphous structure of the particles by comparing the integrated intensity of the background pattern to that of the sharp peaks.

Another useful application is the estimation of the size of the metallic core. As the size of the particle decreases, the peaks of the pattern will broaden. From the broadening it is possible to determine an average crystallite size, in $\AA$, by DebyeScherrer formula: 
$\mathrm{D}_{\mathrm{hkl}}=\mathrm{k} \lambda / \beta \cos \theta$

Where $\mathrm{k}=0.8$-- 1.39 (usually close to unity e.g. 0.9 )

$\lambda$-wavelength of the radiation $\lambda_{\mathrm{Cu}}=1.54056 \AA$,

$\beta$ is the full width at half maximum, or half-width in radians

$\theta$ is the position of the maximum of diffraction.

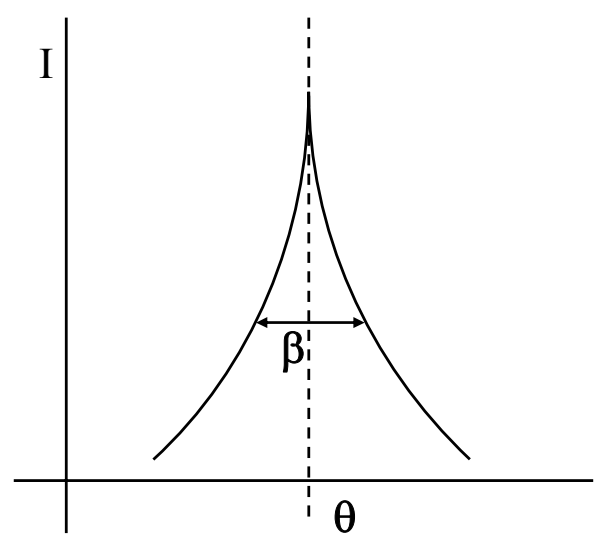

Figure 2.1 Full width at half maximum of Intensity

\subsubsection{X-Ray Photoelectron Spectroscopy (XPS)}

The X-ray photoelectron analysis (XPS) was performed with an Axis Ultra DLD (Kratos Tech.). The spectra were excited by the monochromatized AlK $\alpha$ source $(1486.6 \mathrm{e} . \mathrm{V})$ run at $15 \mathrm{kV}$ and $2 \mathrm{~mA}$. For the individual peak regions, a pass energy of $20 \mathrm{eV}$ was used. Survey spectrum was measured at $120 \mathrm{eV}$ pass energy. Analyses of the peaks were performed with the software provided by the manufacturer, using a weighted sum of Lorentzian and Gaussian components curves after background subtraction. 
XPS, also called Electron Spectroscopy for Chemical Analysis, ESCA, is an electron_spectroscopic method that uses X-rays to eject electrons from inner-shell orbitals. The kinetic energy, $\mathrm{E}_{\mathrm{k}}$, of these photoelectrons is determined by the energy of the $\mathrm{x}$-ray radiation, $\mathrm{h} v$, and the electron binding energy, $\mathrm{E}_{\mathrm{b}}$, as given by:

$E_{k}=h v-E_{b}$

The experimentally measured energies of the photoelectrons are given by:

$E_{k}=h v-E_{b}-E_{w}$

where $E_{w}$ is the work function of the spectrometer. XPS instruments consist of an X-ray source, an energy analyzer for the photoelectrons, and an electron detector. The analysis and detection of photoelectrons requires that the sample be placed in a high-vacuum chamber. Since the photoelectron energy depends on Xray energy, the excitation source must be monochromatic. The energy of the photoelectrons is analysed by an electrostatic analyser, and the photoelectrons are detected by an electron multiplier tube or a multichannel detector, such as a microchannel plate. The energy of the photoelectrons leaving the sample gives a spectrum with a series of photoelectron peaks, being the binding energy of the peaks characteristic of each element. The peak areas can be used, with appropriate sensitivity factors, to determine the composition of the materials surface. And since the shape of each peak and the binding energy can be slightly altered by the chemical state of the emitting atom, XPS can provide chemical bonding information as well. XPS is not sensitive to hydrogen or helium, but can detect all other elements.

In situ low energy Ar etching can be performed in order to probe the desired material, in the case of a core-shell nanoparticle this is specially useful to compare the chemical nature of the core and the external surface of the nanoparticles. The 
etching of the sample is obtained sputtering the surface with an Ar+ ion source (4 $\mathrm{keV}$ energy). In this etching process a depth profiling of around $5 \mathrm{~nm}$ is estimated.

\subsubsection{Dynamic Light Scattering (DLS)}

The particle-size distribution of the composite material was obtained by photon correlation spectroscopy (PCS) measurements (Malvern Zetasizer 3000 HS and Beckman Coulter N5). Particle size analysers determine particle size by measuring the rate of diffusion of particles through a fluid. The rate of diffusion depends on three factors: temperature of the fluid, viscosity of the fluid, and particle size. If the temperature and viscosity are known, the particle size can be determined. Estimation of size by Dynamic Light Scattering is based on two principles: the concept of Brownian Motion and Photon Correlation Spectroscopy.

The motion caused by thermal agitation, that is, the random collision of particles in solution with solvent molecules, is called Brownian motion. These collisions result in random movement that causes suspended particles to diffuse through the solution. For a solution of given viscosity, at a constant temperature, the rate of diffusion or diffusion coefficient (D) is inversely related to the particle size according to the Stokes-Einstein equation:

$$
\mathrm{D}=\mathrm{kT} / 3 \pi \eta \mathrm{d}_{\mathrm{H}}
$$

Where $\mathrm{k}$ is the Boltzmann constant, $\mathrm{T}$ is the absolute temperature, is the solvent viscosity and $\mathrm{d}_{\mathrm{H}}$ is the hydrodynamic size, that is, the size of a particle solvated by liquid molecules in a fluid. The hydrodynamic size or molecular weight of particles in solution is then obtained by measuring the diffusion coefficients of such particles. 
The other principle is Photon Correlation Spectroscopy (PCS). The particles undergoing Brownian motion are detected and analyzed by illuminating the particles with a laser and measuring the scattered light with a photomultiplier tube (PMT). The light scattered by the particles at any given instant adds together to form an interference pattern. The intensity of light detected at the PMT depends on this interference pattern, which in turn depends on the pattern of particles in the laser beam. As the particles move randomly through the solution, their positions relative to one another change, causing a constantly changing interference pattern and varying light intensity at the detector. The intensity changes or fluctuations caused by the Brownian particles, though random in nature, occur on a time scale of microseconds to milliseconds. Large, relatively slowly moving particles, change positions slowly and cause slow intensity fluctuations at the detector; conversely, small, quickly moving particles cause rapid intensity fluctuations.

PCS is based on measuring these scattered-light fluctuations, or fluctuations in the numbers of scattered-light photons. PCS sizes particles by characterizing the exact time scale of the random intensity fluctuations caused by the changing patterns of the diffusing Brownian particles. In order to calculate the diffusion coefficient, and therefore the particle size from the changing light intensity signal, the signal must be mathematically transformed. The result of this transformation is called the autocorrelation function (ACF), which is performed by the size analyser correlator. The time scale of the intensity fluctuations in the light scattered by the diffusing Brownian particles is found by computing the ACF of the scattered-light intensity.

In order to calculate the hydrodynamic size of the particles, two modes of measurement can be utilised: unimodal and size distribution processor (SDP) analysis. Unimodal analysis is a faster method, but it only determines the meanscattering-intensity-weighted particle size and standard deviation of the size 
distribution. Although unimodal results are more accurate for size distributions that are not extremely polydisperse, the mean size provided is still a reliable measure of the sample's mean size even if the true distribution is complex. The standard deviation also provides a good qualitative indication of the polydispersity of the sample, though it too, may not be strictly accurate for complex distributions. On the other hand, SDP analysis determines the particle size distribution. To perform an accurate SDP analysis requires a more accurate ACF than that for unimodal analysis, however, because the decay times of the light fluctuations from all the different sizes of particles must be mathematically separated from a single composite ACF. The accuracy of the correlation function is a function of the length of time over which it is accumulated. If you wish to perform an SDP analysis, a longer run time should therefore be used.

\subsubsection{BET surface area}

For the adequate adsorption and release of a drug or a defined molecule a high surface to volume ratio is required. Gas sorption (both adsorption and desorption) at the clean surface of dry solid powders is the most popular method for determining the surface area of these powders as well as the pore size distribution of porous materials. BET surface areas, $\mathrm{N}_{2}$ adsorption/desorption isotherms, and pore-size distributions of the samples reported in this work were obtained by means of a Micromeritics ASAP 2020 V1 device at $77 \mathrm{~K}$. In a gas sorption experiment, the material is heated and degassed by vacuum force or inert gas purging to remove adsorbed foreign molecules. Controlled doses of an inert gas, such as nitrogen, krypton, or argon, are introduced and the gas is adsorbed, or alternatively, withdrawn and desorbed. The sample material is placed in a vacuum chamber at a constant and very low temperature, usually at the temperature of liquid nitrogen ($195.6{ }^{\circ} \mathrm{C}$ ), and subjected to a wide range of pressures, to generate adsorption and desorption isotherms. The amounts of gas molecules adsorbed or desorbed are 
determined by the pressure variations due to the adsorption or desorption of the gas molecules by the material (the adsorbent). Various amounts of gas molecules will be adsorbed or desorbed at different doses of the gas (the adsorbate). Knowing the area occupied by one adsorbate molecule, $\sigma$ (for example, $\sigma=16.2 \AA^{2}$ for nitrogen), and using an adsorption model, the total surface area of the material can be determined. The most well known and widely used is the Brunauer- EmmettTeller (BET) equation for multilayer adsorption [Brunauer1938]:

$$
\frac{P}{n\left(P_{0}-P\right)}=\frac{1}{c \cdot n_{m}}+\frac{c-1}{c \cdot n_{m}} \frac{P}{P_{0}}
$$

$\mathrm{P}, \mathrm{P}_{\mathrm{O}}, \mathrm{c}, \mathrm{n}, \mathrm{n}_{\mathrm{m}}$ are the adsorption pressure, the saturation vapor pressure, a constant, the amount adsorbed (moles per gram of adsorbent) at the relative pressure $\mathrm{P} / \mathrm{P}_{\mathrm{O}}$, and the monolayer capacity (moles of molecules needed to make a monolayer coverage on the surface of one gram of adsorbent), respectively. Through the slope and intercept of a plot of $\mathrm{P} /\left[\mathrm{n}\left(\mathrm{P}_{\mathrm{O}}-\mathrm{P}\right)\right]$ against $\left(\mathrm{P} / \mathrm{P}_{\mathrm{O}}\right), \mathrm{n}_{\mathrm{m}}$ can be resolved. The specific surface area, $\mathrm{S}$, can then be derived:

$$
S=N_{A} n_{m} \sigma
$$

Where $\mathrm{N}_{\mathrm{A}}$ is Avogadro's number. The specific surface area that can be determined by gas sorption ranges from 0.01 to over $2000 \mathrm{~m}^{2} / \mathrm{g}$. Determination of pore size and pore size distribution of porous materials can be made from the adsorption/desorption isotherm using an assessment model, suitable for the shape and structure of the pores. The range of pore sizes that can be measured using gas sorption is from a few Ångstroms up to about half a micron. 


\subsection{Synthesis}

Magnetic nanoparticles were produced by physical or chemical methods, in order to obtain a range of sizes and properties for different applications. The discharge-arc method and ball milling are top-down methods, i. e. from the bulk starting material we end up in nanoscaled products. These are fast and relatively cheap methods to obtain particles with interesting magnetic properties, like high magnetisation saturations. On the contrary, co-precipitation method is a bottom-up approach, in which a chemical reaction in an aqueous medium forms nanoparticles, that can be further encapsulated in a certain polymer or inorganic coating. Particles thus obtained are smaller and present narrower size distributions. Their production can be optimised to obtain small superparamagnetic nanoparticles, a quality required for a number of applications.

\subsubsection{Discharge-arc}

\subsubsection{Iron encapsulated in carbon.Fe@C.}

The production of carbon coated iron nanoparticles is accomplished by the discharge-arc method designed by Krätschmer-Huffman in 1990. This is a wellknown method for preparing fullerenes and multi-walled carbon nanotubes, and has also been used to develop iron and cobalt nanoparticles coated with carbon layers.

The Krätschmer method uses a cylindrical chamber, in which there are two graphite electrodes: a stationary anode containing 10 microns starting iron powders (ALFA-AESAR), and a moveable graphite cathode (ALDRICH). An arc is produced between the graphite electrodes in a helium atmosphere. The graphite electrode is sublimed and builds up a deposit on the inner surface of the chamber. In the material collected from this deposit we found: carbon nanostructures, 
amorphous carbon and iron and iron oxide nanoparticles encapsulated in graphitic layers.

Non-coated or partially coated magnetic particles, which are not biocompatible, were eliminated by chemical etching. For magnetic purification, stable suspensions of the particles are prepared in a surfactant solution: $2.5 \mathrm{~g}$ of SDS in $500 \mathrm{ml}$ of distilled water. A field gradient produced by $3 \mathrm{KOe}$ permanent magnet was used for magnetic separation of this suspension. The purified material was washed with $\mathrm{HCl} 3 \mathrm{M}$ at $80^{\circ} \mathrm{C}$. The obtained magnetic material was dissolved and the coating carbon forms hydroxil and, in some cases, carboxyl groups, which, due to their hydrophobic nature, contribute to the stability of ferrofluid suspensions. The sample was finally heated at $350^{\circ} \mathrm{C}$ in order to evaporate resulting amorphous carbon. The resulting powders were then filtered

Stable suspensions of the particles were obtained resuspending $1 \mathrm{~g}$ of the particles in $50 \mathrm{mls}$ of the commercially available Gelafundine ${ }^{\circledR}$, a succynilated gel commonly used as a plasmatic subsitute in blood.

\subsubsection{Iron encapsulated in silica.Fe@SiO2}

A modification of the Krätschmer-Huffmann arc-discharge method has been used to produce silica-coated ferromagnetic nanoparticles. In our system the stationary anode is a $4 \mathrm{~mm}$ diameter hollow iron rod containing silica precursor fine powders, and the cathode is a moveable tungsten rod. The whole system is water cooled, the chamber is evacuated, and filled with helium gas. The gas pressure is adjusted to minimise pressure fluctuations during the synthesis. A constant current is driven between the electrodes in contact; they are then slowly separated until an arc is produced between them, ionizing the helium plasma. The system reaches temperatures approximately up to $3000^{\circ} \mathrm{C}$. The iron electrode and the silica powders are then sublimed, iron nuclei condense first and a silica shell is 
formed on the metallic cores, building up a deposit on the inner surface of the chamber. After each preparation, three separate samples were collected from the bottom, the top and the walls of the cylindrical chamber, and their morphological and magnetic properties were studied separately. The magnetic fraction of the sample was separated by means of a magnetic gradient.

Iron rods, purchased form Alfa-Aesar (150 mm, $5 \mathrm{~mm}$ diameter, 99.99\% purity) were drilled to contain the silica powders. As a silica source, commercial Y zeolite with a $\mathrm{SiO}_{2} / \mathrm{Al}_{2} \mathrm{O}_{3}$ ratio of 5.1 and a $900 \mathrm{~m}^{2} / \mathrm{g}$ surface area purchased from Zeolyst International (Valley Forge, PA, USA) was used. Metallic silicon (SigmaAldrich, $99.95 \%$ was also used as a silica source. 6-mm silicon pieces were ball milled under atmospheric pressure for $16 \mathrm{~h}$ to obtain micrometic oxidized silicon powders, which were then placed inside the iron rods and evaporated in the arcdischarge chamber to obtain the final products.

\subsubsection{Mechanical Milling}

\subsubsection{Magnetite embedded in a zeolite matrix. $\mathrm{Fe}_{3} \mathrm{O}_{4} @ \mathrm{ZY}$}

Commercial $\mathrm{NaY}$ zeolite, purchased from Zeolyst International (Valley Forge, $\mathrm{PA}, \mathrm{USA}$ ) with a $\mathrm{SiO}_{2} / \mathrm{Al}_{2} \mathrm{O}_{3}$ ratio of $5.1,900 \mathrm{~m}^{2} / \mathrm{g}$ surface area, and $\sim 1 \mu \mathrm{m}$ crystal size (measured by SEM, result not shown) was first calcined at $753 \mathrm{~K}$ in order to remove any rest of organic template used during the hydrothermal synthesis, and then milled together with commercial magnetite nanopowder (Sigma-Aldrich, $>98 \%, 20-30 \mathrm{~nm}$ particle size) using a zeolite/magnetite ratio of $3: 1$. The mixture zeolite/magnetite was ground through the combination of impact and friction in a Fritsch Mill (model: Anlysette) under a vacuum of $1.10^{-4} \mathrm{mbar}$ for $16 \mathrm{~h}$ at room temperature. 


\subsubsection{Co-precipitarion and Sol-Gel}

2.2.3.1 Magnetite encapsulated in silica. $\mathrm{Fe}_{3} \mathrm{O}_{4} @ \mathrm{SiO}_{2}$

$\mathrm{FeCl}_{3} \cdot 6 \mathrm{H}_{2} \mathrm{O}, \mathrm{FeCl}_{2} \cdot 4 \mathrm{H}_{2} \mathrm{O}$, ethanol, ammonia aqueous (25\% wt.), tetraethyl ortosilicate (TEOS), 3-aminopropyltriethoxysilane, toluene anhydrous, dimethylformamide, glutaric anhydride, imidazole, MES buffer, ethyl-3-(3dimethylaminopropyl)-carbodiimide (EDC), and N-hydroxy succinimide (NHS) were purchased from Sigma-Aldrich and used as supplied. Ultra pure water was used throughout the experiments (MilliQ system, Millipore).

Magnetite nanoparticles were prepared following the coprecipitation method described by Ma et al. [Ma2006]. A basic solution, i.e. ammonia, was slowly added from an addition funnel to a mixture of $\mathrm{FeCl}_{2} \cdot 4 \mathrm{H}_{2} \mathrm{O} \quad 0.33 \mathrm{M}$ and $\mathrm{FeCl}_{3}-6 \mathrm{H}_{2} 0$. The particles thus obtained were washed three times with distilled water, separated with a magnet, centrifuged and dried in an oven at $60^{\circ} \mathrm{C}$. The size of the particles depends on a number of factors. The basic solution used restrains the size of the nuclei formed, being the smallest particles obtained when $\mathrm{NH}_{4} \mathrm{OH}$ is used as the solution; the size increases as $\mathrm{KOH}$ or $\mathrm{NaOH}$ are used in the synthesis. A vigorous stirring during the process and a very slow addition of the base are the key to obtain homogeneous particles. However there is always a dispersity of sizes obtained due to the preparation, and further purification and separation are required nevertheless.

The silica shell was synthesized using the sol-gel procedure optimized by Deng et al. [Deng2005] using Tetraorthosilicate (TEOS) as silica source in a basic ethanol/water mixture at room temperature. $2 \mathrm{~g}$ of a $2 \%$ suspension of the magnetite nanoparticles in distilled water was added to a mixture of $160 \mathrm{ml}$ of $\mathrm{EtOH}$ and $40 \mathrm{ml}$ of $\mathrm{H}_{2} \mathrm{O}$. Then, $1 \mathrm{ml}$ of TEOS was added dropwise to the solution and the reaction was kept at room temperature and vigorous stirring for $12 \mathrm{~h}$, after which 
the resulting particles were thoroughly washed and separated magnetically for their complete characterization.

\subsection{Functionalisation}

The binding of functional groups on the surface to enhance the reactivity of the particles towards certain biomolecules in solution is described in this section.

\subsubsection{Surface functionalisation: Chemical coupling}

The surface of carbon and silica has hydroxyl groups that can be substituted with other molecules in order to enhance their reactivity. Amine, carboxyl and chloride groups can be introduced by the reaction with the corresponding silane in an organic solvent like toluene or THF.

\subsubsection{Functionalisation with amine groups}

Surface functionalization of the silica coatings with amine and carboxyl groups was carried out following the experimental procedures of Ha et al, and Levy

et al., respectively. 3-aminopropyl was the covalent linker used to attach amine functional groups on the external surface of the silica shells. The amine functionalized nanoparticles were prepared by boiling a toluene solution dissolved with 3-aminopropyltriethoxysilane (APTES) and refluxed under argon for $3 \mathrm{~h}$ to tether the 3-aminopropyl groups on the external surface of the silica coated nanoparticles.

\subsubsection{Functionalisation with carboxyl groups}

The carboxyl-functionalized nanoparticles were prepared by dispersing the composite nanoparticles, obtained after the treatment in a reflux of toluene with APTES, in a mixture of 3-aminopropyltriethoxysilane and glutaric anhydride 
dissolved in dimethylformamide (DMF) during $24 \mathrm{~h}$. The particles thus obtained were repeatedly washed with acetone first, and mixtures of acetone/water in which the ratio of organic/aqueous ratio was consecutively diminished, in order to efficiently remove all traces of organic solvent.

\subsubsection{Coupling of antibodies}

In order to develop a contrast agent with potential active targeting properties, carbon and silica-coated magnetic nanoparticles have been functionalised with an antibody. The antibody anti-hCG was chosen because it is widely studied due to its common use in pregnancy testing kits. Antibodies are immune system-related proteins, which consist of four polypeptides: two heavy chains and two light chains-joined to form a "Y" shaped molecule (see Fig. 4.5, chapter 4). They present amine and carboxyl terminal groups that can be used to bind our aminated or carboxylated particles through a glutaraldehyde or carbodiimide covalent bond, respectively.

\subsubsection{Carbodiimide}

The covalent coupling of the antibody (anti-hCG) to the carboxylfunctionalized nanoparticles was carried out following a standard protocol with water soluble carbodiimide (EDC) [Bangs2007]. After covalent coupling the nanoparticles were re-suspended in a storage buffer composed of Tris/ $\mathrm{HCl}, 10 \mathrm{mM}$ at $\mathrm{pH} 8.1$ and $1 \% \mathrm{wt}$.

\subsubsection{Glutaraldehyde}

The covalent coupling of the antibody (anti-hCG) to the amino-functionalized nanoparticles was carried out with an excess of glutaraldehyde as the amine reactive homobifunctional cross-linker in a phosphate-buffered saline (PBS) buffer 
as the washing/coupling buffer. After immersion in PBS, the initial nanoparticle concentration was $10 \mathrm{mg} / \mathrm{mL}$. The obtained pellet was dispersed in $10 \mathrm{~mL}$ of a 10 wt. \% glutaraldehyde solution. $0.15 \mathrm{mg}$ of antibody were dissolved in $1 \mathrm{~mL}$ of coupling buffer. The nanoparticle suspension was combined with the antibody solution and reacted for $4 \mathrm{~h}$ at room temperature with continuous mixing. The obtained product was washed gently and magnetically separated. After covalent coupling the nanoparticles were re-suspended in a storage buffer composed of Tris/HCl, $10 \mathrm{mM}$ at $\mathrm{pH} 8.1$ and 1 wt. \%.

\subsubsection{Verification of the antibody coupling}

Both for carbodiimide and glutaraldehyde binding, the suitable attachment of the antibody to the magnetic nanoparticles was verified. For this purpose an immunochromatographic assay (also termed lateral flow test) was carried out, using the attachment of the conjugate particle-antibody to the human chorionic gonadotropin hormone and subsequent detection in a nitrocellulose membrane, where the complementary anti-antibody was previously immobilized. The specific binding streptavidin-biotin is used as the control line in the membrane to assure the quality of the test.

To attain detection of the antibody-conjugated nanoparticles $2 \mu \mathrm{L}$ of the $1 \mathrm{wt}$. $\%$ nanoparticles dispersion were mixed with increasing amounts of the hCG hormone in a buffer composed of: phosphate buffer $10 \mathrm{mM}, \mathrm{NaCl} 150 \mathrm{mM}$, BSA 0.3 wt. $\%$, Tween-20 0.1 wt. $\%$, and sodium azide 0.1 wt. $\%$ at $\mathrm{pH}$ 7.4. Monoclonal anti-Chorionic Gonadotropin ( $\alpha+\beta$ subunits) (hCG) antibody was purchased from Medix Biochemica (Finland). One end of the test strip was immersed in this suspension and then the elution flow proceeded by capillarity. Capture of the antibody-functionalized nanoparticles produced a dark band in the region of the test strip where the complementary anti-antibody had been immobilized. The 
intensity of colour can be photographically assessed and related to the concentration of nanoparticles in the suspension and with the amount of hCG in the solution.

A control assay is also performed using antibody-conjugated nanoparticles without adding the hCG hormone to the buffer dispersion. Without the hormone in the media no band was detected (optically or magnetically), showing that there is a specific interaction between the hormone and the anti-hCG antibodies.

\subsubsection{Pegylated particles}

In order to avoid the action of the immune system and prolonge their stability in aqueous suspensions and therefore their residence time in blood, $\mathrm{Fe} @ \mathrm{C}$ particles were coated with an hydrophilic PEG layer. The amphiphilic copolymer PMAOPEG was obtained following the procedure by Yu et al [Yu2006]. $30 \mathrm{mg}$ of Poly (maleic anhydride-alt-1-octadecene) (PMAO, Mw=50000, ALDRICH) and $90 \mathrm{mg}$ of primary amino group terminated poly(ethylene glycol)methoxy ether (mPEG$\mathrm{NH} 2, \mathrm{Mw}=5000, \mathrm{ALDRICH})$ were mixed in $5 \mathrm{mls}$ of chloroform $\left(\mathrm{CHCl}_{3}\right)$ and stirred at room temperature overnight in a molar ratio of $1-30$. After that, a $20 \mathrm{mg}$ of $\mathrm{Fe}-\mathrm{C}$ particles suspension in $5 \mathrm{ml}$ of $\mathrm{CHCl}_{3}$ was sonicated with an ultrasonic probe for 5 minutes and the stable suspension was added to the obtained copolymer. The mixture was sonicated for $15 \mathrm{~min}$ in an ultrasound bath and left to react at room temperature for $48 \mathrm{~h}$. After this time the magnetic solid was separated with a magnet and the liquid decanted. The organic solvent was thoroughly removed by washing with ethanol, acetone, and finally distilled water. 


\subsection{In-vitro experiments}

\subsubsection{Haematology results}

The study of hemorheological parameters has been recommended for biocompatibility testing. Conventional radiological contrast media and those involving paramagnetic nanoparticles used in nuclear resonance are usually tested for viscosity. We have performed the basic recommended tests adapting them to nanoparticles in circulation.

To prepare the biocompatible suspensions, 100 mg of Fe@C nanoparticles produced by the arc-discharge method were suspended in $100 \mathrm{ml}$ of Gelafundine ${ }^{\circ}$, a commercial succinylated gel commonly used as plasmatic substitute of blood. The solution was then sonicated for fifteen minutes. Human blood from 5 healthy volunteer donors was tested in vitro, before and after being mixed with the particles suspension in different dilutions $(1 \mathrm{ml}$ of blood and $0.06 ; 0.12 ; 0.24$ and $0.5 \mathrm{ml}$ of particles). The same procedure was followed for the tests in animals (ex-vivo tests): 10 non-injected New Zealand rabbits (as control) and other 10 animals (test rabbits) after intravenous injection of $1 \mathrm{ml}$ of the particles suspension in Gelafundine ${ }^{\circledR}$. Blood samples were taken $10^{\prime}, 30^{\prime}$ and 24 hours after the injection of particles.

Blood viscosity was measured with a Brookfield ${ }^{\circledR}$ DV-3 cone-plate viscometer at different shear stress (230 s-1, $23 \mathrm{~s}-1$ y $5.7 \mathrm{~s}-1)$, and plasma viscosity with a capillary viscometer Fresenius ${ }^{\circledR}$. We have determined as well erythrocyte aggregability in vitro with a Mirenne ${ }^{\circledR}$ aggregometer after 5 and 10 seconds of stasis and after 5 and 10 seconds of very low shear stress (3s-1). The shear stress applied in this experiment simulates those of circulating blood in different areas of circulation: veins, capillaries and arteries. We have done as well a complete blood 
count, including differential leukocyte count, with a haematology cell counter (Coulter LH-750®) and the usual coagulation tests: Prothrombin time, thromboplastine time and fibrinogen (ACL-9000® coagulation analyzer and Hemosil IL $®$ kit), and D-Dimer immuno-turbidimetry (Turbiquant D-Dimer DadeBehring $\left.{ }^{\circledR}\right)$.

\subsubsection{Uptake by dendritic cells}

Dendritic Cells (DCs) are the main antigen presenting cells of the immune system. The inclusion of $\mathrm{Fe} @ \mathrm{C}$ particles inside DCs has been studied with the aim of reaching a tumour site and release a chemotherapy agent or selectively heat and kill malignant cells by means of an alternate magnetic field.

\subsubsection{Cell culture}

Mononuclear cells obtained from $14 \mathrm{ml}$ of blood were cultivated during seven days with AIM-V media with Interleukin $4\left(10 \mathrm{U}^{5} / 100 \mathrm{ml}\right)$ and Granulocytemacrophage colony stimulation factor $\left(10 \mathrm{U}^{5} / 100 \mathrm{ml}\right)$. On day five $5 \mu \mathrm{g} / \mathrm{ml}$ of media containing lypopolysacharides were added to induce maturation of DCs. On day 7 $15 \mu \mathrm{l} / \mathrm{ml}$ of media of a $5 \%$ suspension of Fe-C nanoparticles were added to the culture. Finally cells were recollected on day 8, washed twice with PBS and resuspended in the same buffer and centrifuged during $25 \mathrm{~min}$ at $400 \mathrm{G}$ with Ficoll histopaque $(1.077 \mathrm{~g} / \mathrm{ml})$ DCs were isolated from the PBS/Ficoll interface and unincorporated NPs remained in the bottom of the tube. Incorporation of MNP was assessed by confocal and electronic microscopy. 


\subsubsection{Viability Studies}

DCs were cultured in a 12 wells plate and NPs incorporated as previously described and cells were recollected from different plates on days 8, 9, 10, 11 and 12 and viability of the DCs was assessed with tripan blue.

\subsubsection{Electron Microscopy}

Electron Microscopy was done in the Anatomy Service of the University of Zaragoza. Cells were fixed with glutataraldehyde and made resistance with lead nitrate.

\subsubsection{Kinetics studies of drug adsorption and release}

Doxorubicin hydrochloride injectable solution was purchased from Pharmacia Spain. Doxorubicin adsorption was performed by dispersing $10 \mathrm{mg}$ of nanoparticles in $4 \mathrm{~mL}$ of doxorubicin solution $(0.2 \mathrm{mg} / \mathrm{mL})$ until saturation following the experimental procedure described by Kuznetsov et al [Kuznetsov1999]. At fixed time intervals, the magnetic nanoparticles were separated from the supernatant by means of a permanent magnet. $1 \mathrm{~mL}$ of supernatant was withdrawn from the vial and measured by UV spectrometry at 498 nm (Varian Cary-100 Bio spectrophotometer). After each measurement each sample was reincorporated into the vial.

After reaching saturation, the drug-loaded magnetic nanoparticles were washed three times in distilled water to remove all the non-adsorbed doxorubicin, magnetically separated by means of a permanent magnet, and dried. The release profile was obtained by dispersing the dried drug-loaded nanoparticles in $4 \mathrm{ml}$ of human plasma kept at $37^{\circ} \mathrm{C}$. At fixed time intervals, the magnetic nanoparticles were separated from the supernatant by means of a permanent magnet. A small 
volume of supernatant was withdrawn from the vial and measured by UV spectrometry at $498 \mathrm{~nm}$. After each measurement the vial was refilled with plasma to keep the volume constant, and the new dilution was recalculated for each measurement.

\subsection{In-vivo experiments}

The particles were tested in an in-vivo preclinical animal model. Magnetic implants were inserted in the main organs of New Zealand rabbits and the capability of the magnet to attract nanoparticles was tested. The design of the experimental procedure was previously evaluated by the Ethics Committee for Animal Research of the University of Zaragoza, code PI 19/04. To achieve the targeted delivery of particles to the implanted organ three different strategies were designed: intravenous injection of the particles through a marginal ear vein of the animal, intrarterial injection and direct inoculation of the particles suspension under echographic control.

\subsubsection{Preclinical experimental model}

Stable suspensions of the carbon coated iron nanoparticles were tested in an in-vivo preclinical animal model. Magnetic implants were inserted in the main organs of 36 New Zealand rabbits and the capability of the magnet to attract the magnetic particles was tested. The experimental procedure was previously evaluated by the Ethics Committee for Animal Research of the University of Zaragoza, code PI 19/04 


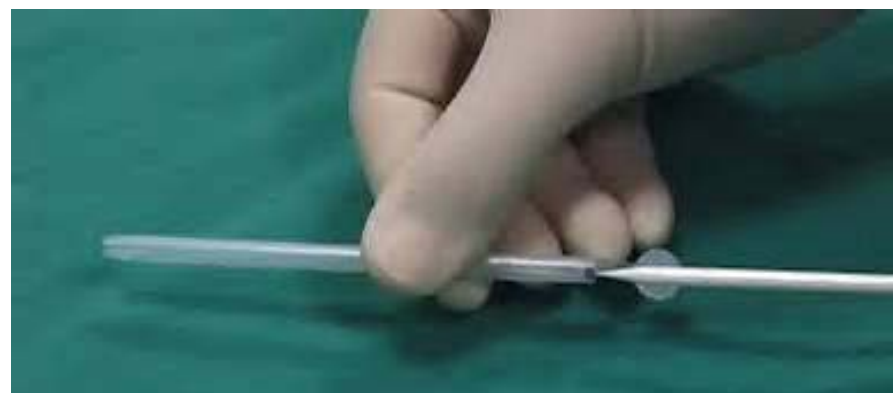

Figure 2.2 French plastic trocar with a sharpened tip, used to perforate the desred organ and insert the small magnetic implant.

The animals, after 12 hours without being fed, were premedicated with an intramuscle Medetomidine (Domtor ${ }^{\circledR}$ ) $10 \mathrm{mg} / \mathrm{Kg}+$ Ketamine (Imalgene ${ }^{\circledR}$ 500) 20 $\mathrm{mg} / \mathrm{Kg}$ injection. Only when it was necessary Atropine $0.05 \mathrm{mg}$ was added complementarily. A 22 gauge Braunüle Vasocan Catheter was inserted in the marginal vein of the ear, and a perfusion of a physiologic saline solution was initiated. Next, the peritoneal cavity was insufflated with $\mathrm{CO}_{2}$ through a Veress needle and a minilaparoscopic optic was introduced using a $5 \mathrm{~mm}$ trocar. Then, through an 8 French plastic trocar (Fig. 2.2), and under endoscopic control, the lower pole of the left kidney was punctured, inserting a 4x2 mm cylindrical permanent magnet (neodymium-iron-boron coated with gold) in it (Fig. 2.3). The right kidney was also punctured but no magnet was inserted. The purpose is to reproduce the same tissue damage as in the counter lateral kidney, and to have a control organ to compare the concentration of the particles attracted to the targeted organ. After controlling the absence of bleeding, the trocars were retired and the cutaneous insertion orifices were sutured. 


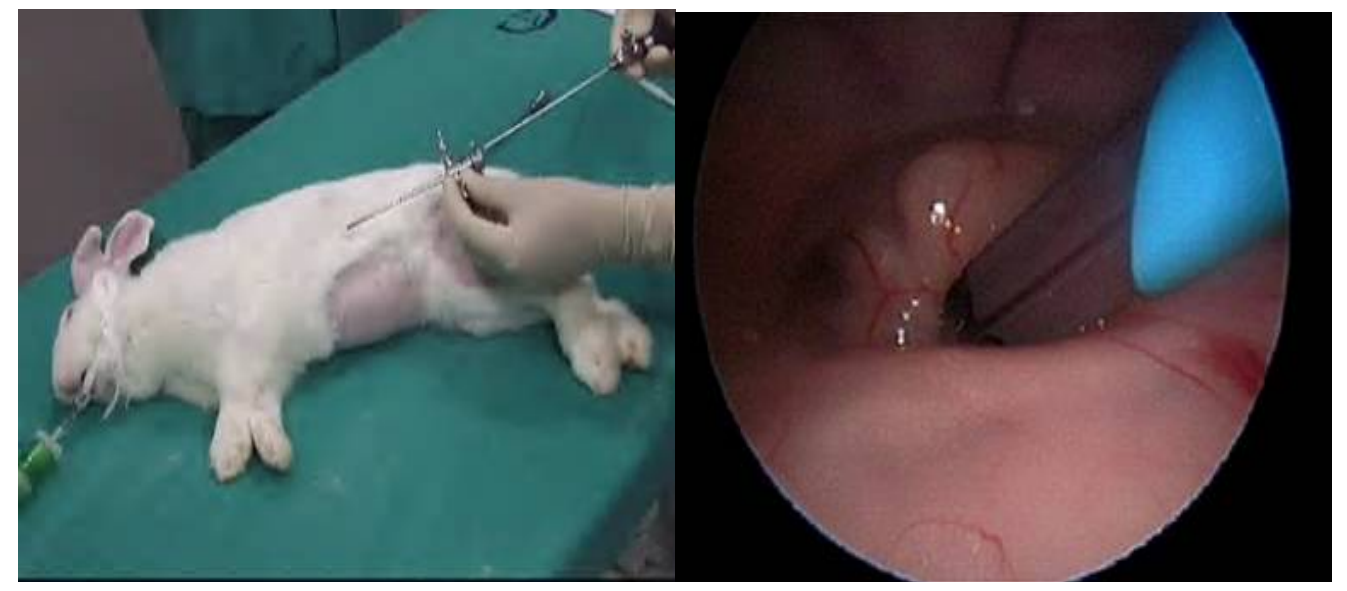

Figure 2.3 Under laparoscopic control, the kidney is punctured and the magnetic implant is inserted.

\subsubsection{Intravenous administration}

$2 \mathrm{ml}$ of the particles suspension were injected intravenously in the marginal ear vein of the rabbit. Different groups of animals were sacrificed by terminal anaesthesia after half an hour, 2, 7, 14, 21 and 28 days, and the main organs (lungs, liver, spleen, kidneys and spine) were extracted for histological study.

\subsubsection{Direct injection in the affected organ}

A week after the insertion of the magnet, nanoparticles are directly injected into the kidney under ultrasound control. General anaesthesia was induced administering $0.4 \mathrm{mg}$ of medetomidine (Domtor ${ }^{\circledR}$ ) and $60 \mathrm{mg}$ of ketamine (Imalgene 1000®) intramuscularly. The left flank was shaved and the zone disinfected with a 50\% mixture of iodine and ethanol. The guide for biopsy needles was set in the probe of the ultrasound scanner Sonosite Titan ${ }^{\circledR}$ with a $20 \mathrm{G}$ adaptor. The probe was aimed towards the kidney in which a magnet had been inserted. 
The magnet, a solid and metallic object inside the kidney, produces the apparition of an artifact called "comet tail". The ultrasound beam contacts a narrow and echogenic interface and as a consequence a series of linear echoes appear.

Once the magnet has been identified, the route of penetration of the needle appears on the screen so that the position of the magnet can be centred. Once the route of injection is made to coincide with the magnet's position, a $20 \mathrm{G}$ calibre and $70 \mathrm{~mm}$ long needle is inserted and the load of nanoparticles is inoculated, after which the needle is removed. 


\section{Chapter 3}

\section{Characterisation}

Four novel preparations of core-shell magnetic nanoparticles are presented in this work. First, iron nanoparticles encapsulated in carbon and silica have been produced by the discharge-arc method [F.-Pacheco2006, F.-Pacheco2007]. The mechanical milling of magnetite and zeolite yields a composite material containing magnetite nanoparticles embedded inside zeolite matrixes [Arruebo2006b]. And by a combination of the co-precipitation method and the sol-gel technique small magnetite crystallites have been encapsulated in amorphous silica [Arruebo2007b]. The morphological, physical and chemical properties can make each particle more suitable for a certain application, either in-vitro or in-vivo, depending on their size, magnetic properties, surface area and functional groups on the surface. In order to organise data in a more suitable way the characterisation of the materials is presented separately for each type of particle.

\subsection{Iron coated by carbon. Fe@C}

With a slight modification of the Krätschmer-Huffman discharge-arc chamber, iron nanoparticles coated by graphitic layers have been obtained. The relatively low price of the initial reagents and the availability of the arc furnace make it possible to produce big quantities of raw materials. After magnetic purification and successive filtration fine black powders are obtained. 


\subsubsection{Transmission Electron Microscopy (TEM) and XRD}

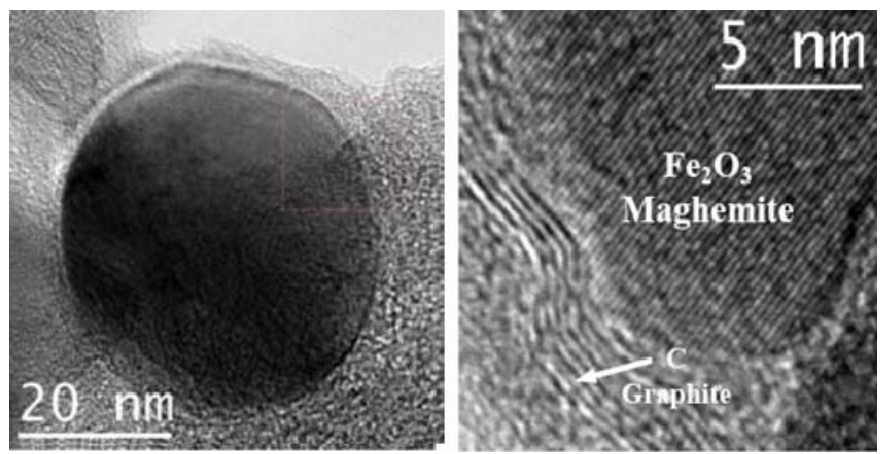

Figure 3.1 HRTEM images of two different $\gamma-\mathrm{Fe}_{2} \mathrm{O}_{3}$ nanoparticles encapsulated in graphitic layers. On both images a perfect encapsulation of the magnetic core by graphitic layers can be observed.

High-resolution TEM (HRTEM) and Energy Filtered TEM (EFTEM) images show that the coating of the iron particles is complete, thus rendering them biocompatible. Fe appears with a darker contrast whereas $\mathrm{C}$, a lighter element, appears with a lighter contrast in Fig. 3.1.
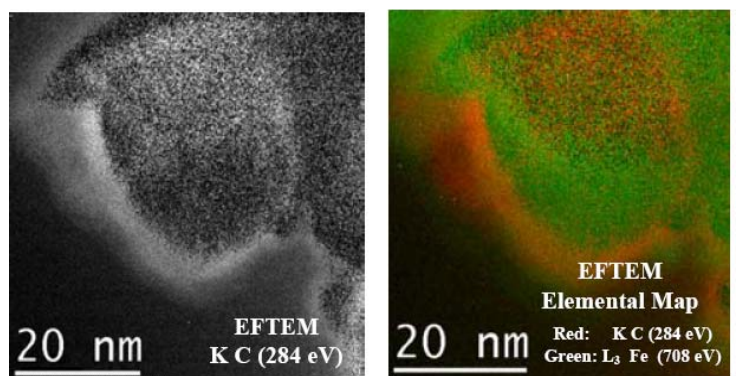

Figure 3.2 EFTEM image composition of a Fe@C nanoparticle. Red corresponds to graphitic encapsulation, whereas green represents the presence of the Fe metallic core.

Moreover HRTEM micrographs showed that the plane spacing observed in that specific nanoparticle correspond to the $\gamma-\mathrm{Fe}_{2} \mathrm{O}_{3}$ Maghemite phase, whereas the plane observed at the encapsulation layer should be associated with the $\mathrm{C}$ graphite 
phase. For the EFTEM images (Fig.3.2), iron (EELS peak at $708 \mathrm{eV}, \mathrm{L} 3$ peak) and carbon (EELS peak at 228, K peak) were selected separately. Once both images were obtained, an elementary map can be drawn, showing a coloured distribution of each of the elements: green for Fe and red for $\mathrm{C}$.

Furthermore, sample composition could be also analysed by electron diffraction (Fig. 3.3). The obtained results were in good agreement with those previously obtained by HRTEM. Point symmetry and lattice spacing of iron oxide in the core of the nanoparticle was observed (cubic phase of the $\gamma-\mathrm{Fe}_{2} \mathrm{O}_{3}$, Maghemite).

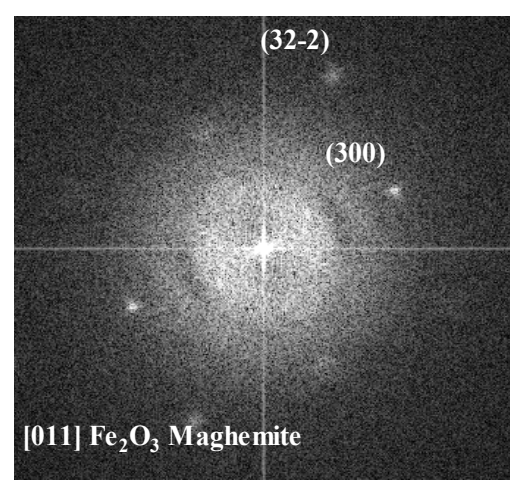

Fig. 3.3 Electron Diffraction proves that the particle core of the analysed particle crystallises in the maghemite phase.

Figure 3.4 is the image of another particle observed with an Ultra High Resolution Transmission Electron Microscope (UHRTEM Titan 80-300). In this case the metallic core is crystallised in the $\alpha$-Fe phase, as it can be observed from the distances between atomic planes, much smaller than in the case of iron oxides. The presence of iron nanoparticles is predominant for all preparations. 


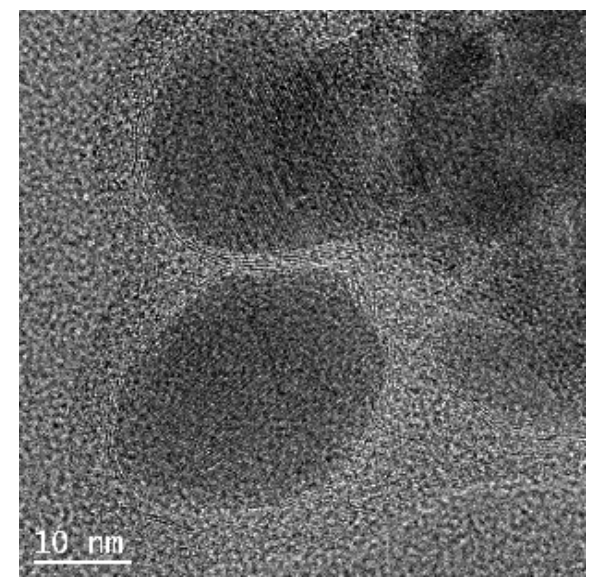

Figure 3.4 Ultra High Resolution Transmission Electron Microscopy (UHRTEM Titan 80300) image of an iron nanoparticle encapsulated in carbon. Atomic planes of the metallic core and the graphitic coating are clearly visible.

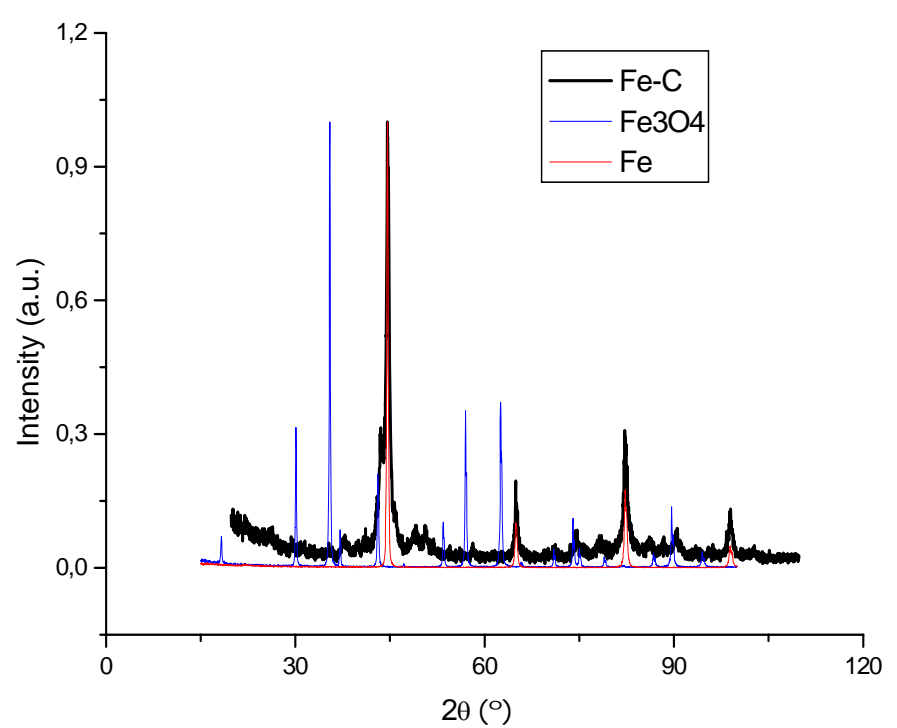

Figure 3.5 XR diffractogram of the Fe@C nanoparticles. Experimental diffractogram (black) peaks correspond to those of Iron (red), whereas the proportion of iron oxides (blue, magnetite) is much smaller.

This is also checked by X-Ray Difrraction. XR diffractogram proves that most 
of the material is crystallised in the $\mathrm{Fe}^{0}$ phase, without undergoing oxidation (Fig. 3.5). The apparition of some very small particles oxidised to maghemite can be due to processes of oxidation that take place during the synthesis of the particles at the discharge-arc chamber, and that is what can be observed by TEM analysis, although those peaks are not significant in the diffractogram of the sample, because the fraction of these small particles is really small.

From TEM images, the size distribution of the particles can be analysed. Different images from different regions of a same sample area analysed, and the particles' diameter measured, to obtain a size distribution histogram that can be fitted to a log normal distribution (Fig. 3.6). Whereas some particles bigger than 40 $\mathrm{nm}$ could observed, most of the analysed material was distributed between 5 and 23 $\mathrm{nm}$. Maximum is centred in $10 \mathrm{~nm}$. The sample preparation for TEM analysis is an inconvenient for the accurate size measurement, since only smaller particles are able to be penetrated by electrons, and therefore bigger particles or aggregates are left out.

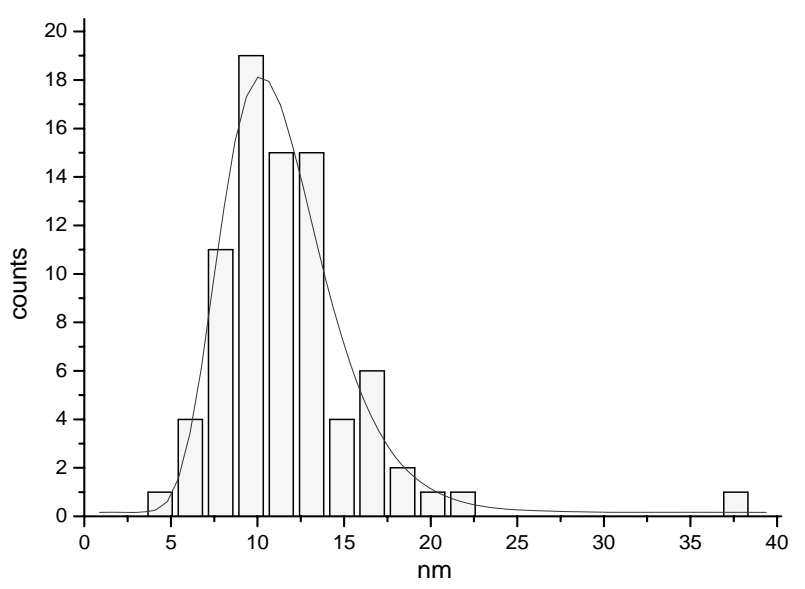

Figure 3.6 Histogram of particles size from TEM images. Slightly broad size dispersion is observed, from 5 to $40 \mathrm{~nm}$. Maximum is centred in $10 \mathrm{~nm}$. 
3.1.2 Dynamic Light Scattering (DLS). Estimation of size.

DLS was performed for all the samples, and the obtained average size was 200 $\mathrm{nm}$ (Fig. 3.7). DLS Measurements provide us information about the hydrodynamic radius of the particle, i.e. the size of the particle in a liquid suspension, surrounded by the solvent molecules. It is expected that DLS yields a particle size different to the one obtained by direct TEM analysis: first of all TEM is a local measurement and DLS measures the sample as a whole; moreover the behaviour of particulate materials in solution or in a liquid suspension is strongly different to their behaviour as solids. On the other hand, TEM measures only the fraction of the smallest nanoparticles. Particles (or aggregates of particles) bigger than $150 \mathrm{~nm}$ are opaque to the electron beam, as electrons are not able to penetrate inside such big particles. In addition, sample preparation is made by acquiring a drop from a suspension of the iron oxide coated powder into n-hexane. Big nanoparticles tend to be deposited onto the bottom side of the solution just by gravity, and drop taken from the top-middle solution always underestimate the particle size.

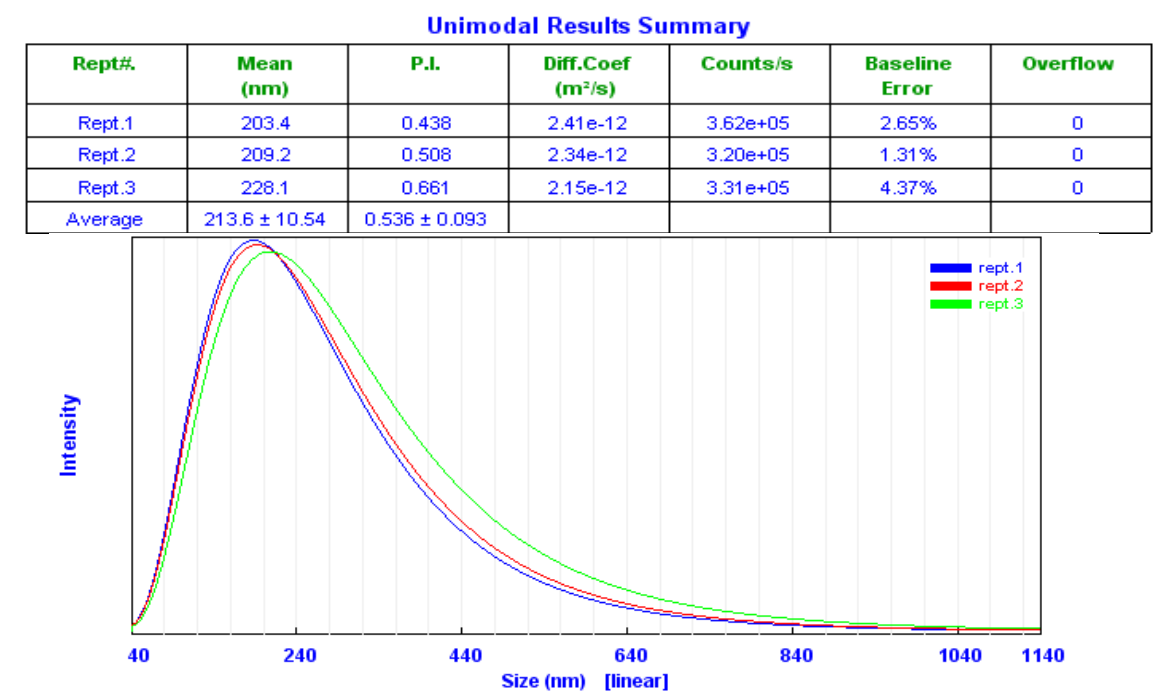

Fig. 3.7 DLS estimation of the hydrodynamic radius of the Fe@C particles in aqueous suspension. A broad distribution is observed, and the maximum is centred on $200 \mathrm{~nm}$. 


\subsubsection{Magnetic Properties}

The magnetisation isotherms can be understood as the superposition of two different magnetic behaviours, consistent with the existence of two different particle size ranges in our samples (Fig. 3.8). The largest particles give rise to a high saturation magnetisation and coercivity is observed, even at room temperature. This suggests the existence of ferromagnetic aggregates of particles (several hundreds of nanometers, in good agreement with the DLS results). Considering this particle size, one can expect in our sample a blocking temperature above room temperature. This fact is confirmed by field cooling (FC) and zerofield cooling (ZFC) magnetisation measurements (Fig. 3.9). On the other hand, the high field susceptibility displayed by the hysteresis loop is due to the existence of small particles (tens of nanometers, according to the TEM results), showing a superparamagnetic behaviour.

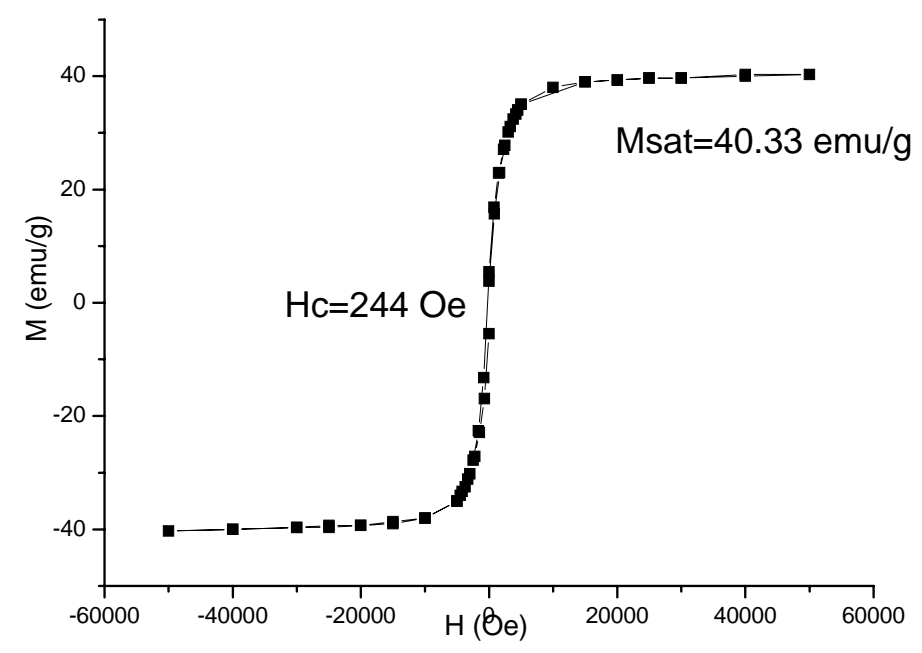

Fig. 3.8 Hysteresis loop of the Fe@C particles. Ferromagnetic behaviour is observed. 


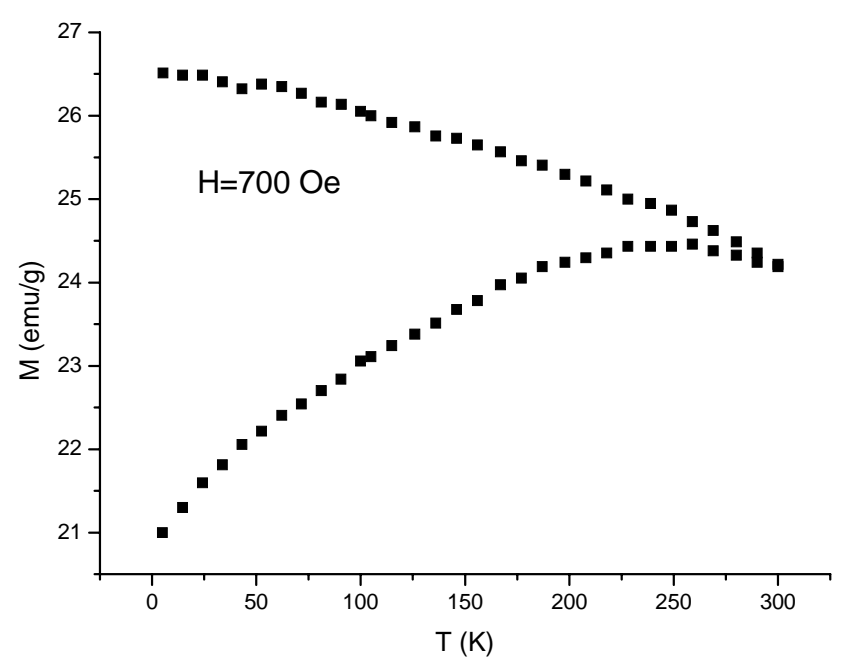

Fig. 3.9 Zero Field Cool and Field Cool measurements confirm the presence of blocked aggregates of particles for the whole range of temperatures. 


\subsection{Iron coated by silica. Fe@SiO}

Silica-encapsulated iron nanoparticles were obtained using the arc-discharge system. Modifying the Krätschmer-Huffmann method mostly isolated and homogeneous particles have been obtained, as proved by TEM and XRD measurements. An iron rod was used as the anode, and Y-zeolite or oxidized metallic silicon powders were used as the silica source. These particles combine the high magnetisation saturation and strong response to magnetic fields of their ferromagnetic core with the adsorption properties and chemical versatility of their silica coating, presenting a reasonable BET surface area for the adsorption of drugs or biomolecules.

Elementary analysis (ICP-AES) gave an average amount of $12.6 \pm 0.5 \mathrm{wt} \%$ of Silica per total mass of sample, which is in agreement with the initial amount of reactants used for the synthesis of iron-silica products.

\subsubsection{Magnetic Properties}

The room temperature magnetisation values obtained is the largest reported for magnetic nanoparticles encapsulated in inorganic matrices (see a revision of literature results in the Appendix to this chapter). The magnetic moment values are given per unit of total mass $(\mathrm{emu} / \mathrm{g})$,=considering the total weight of both, silica and iron in the nanoparticles. Different samples reach values over $160 \mathrm{emu} / \mathrm{g}$, and $162.9 \mathrm{emu} / \mathrm{g}$ is the maximum magnetisation found at 3 Tesla. The closest result, $140 \mathrm{emu} / \mathrm{g}$, was reported by Tang et al. [Tang2005], for cobalt-silica composites produced by the sol-gel method. All the samples showed ferromagnetic behavior, attaining saturation at $2 \mathrm{~T}$ (Fig. 3.10). Coercivities around 350 Oe were obtained in all cases when Zeolite $\mathrm{Y}$ was used as the silica source, increasing to 479 Oe when metallic silicon was used as precursor. The diamagnetic behavior of $\mathrm{SiO}_{2}$ is 
responsible for the lower value of magnetisation with respect to that of metallic iron, from $217.6 \mathrm{emu} / \mathrm{g}$ of pure iron [Crangle1971] to $162.9 \mathrm{emu} / \mathrm{g}$ of our material.

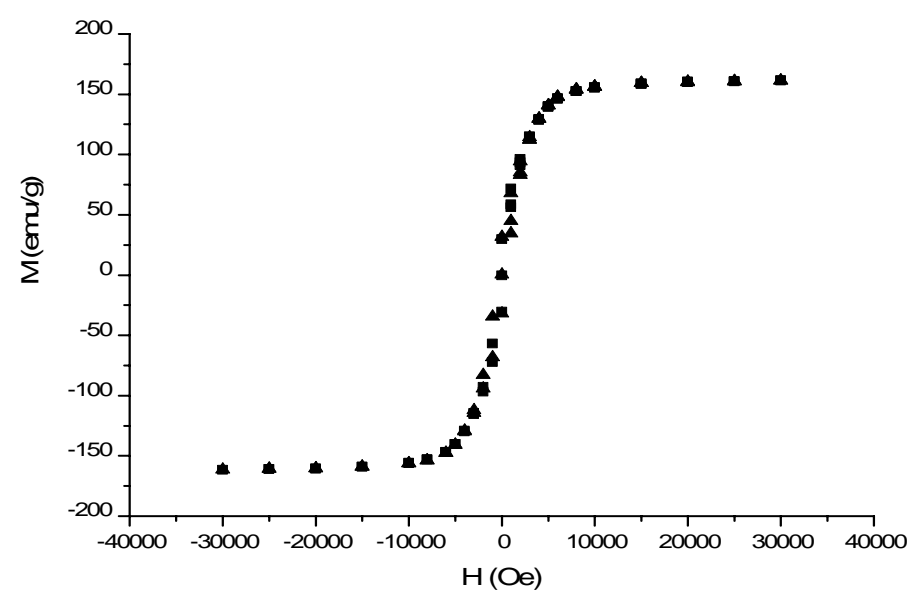

Fig. 3.10 M Vs H isotherm at $300 \mathrm{~K}$ for the $\mathrm{Fe} @ \mathrm{SiO} 2$ particles produced by the dischargearc method when $\mathrm{Y}$ zeolite (squares) or metallic silicon (triangles) is used as the silica precursor, respectively. The high coercivity (340 Oe) and magnetic saturation (160.1 $\mathrm{emu} / \mathrm{g}$ ) values confirm the ferromagnetic behaviour and the strong magnetic response of both samples.

\subsubsection{Transmission Electron Microscopy}

When Y zeolite was used as the silica source, TEM images of the obtained samples prove the presence of iron nanoparticles well coated by a quasi-amorphous silica matrix (Figs. 3.11.A, 3.11.B). The particles are coated with a zeolite-type aluminum silicate that decomposes under the electron beam, as zeolites are very electrosensitive materials that amorphisise quickly under the electron beam: in some areas atomic planes that disappear after some seconds of exposition to the electron beam were observed. EELS spectra obtained from the cores show the clear presence of iron, while the shell is mainly made of silicon and oxygen, as it can be observed by EFTEM (fig. 3.11.D). HRTEM images also show that the atomic 
interspaces found for the particle cores correspond to those of $\alpha$-Fe (metallic phase).
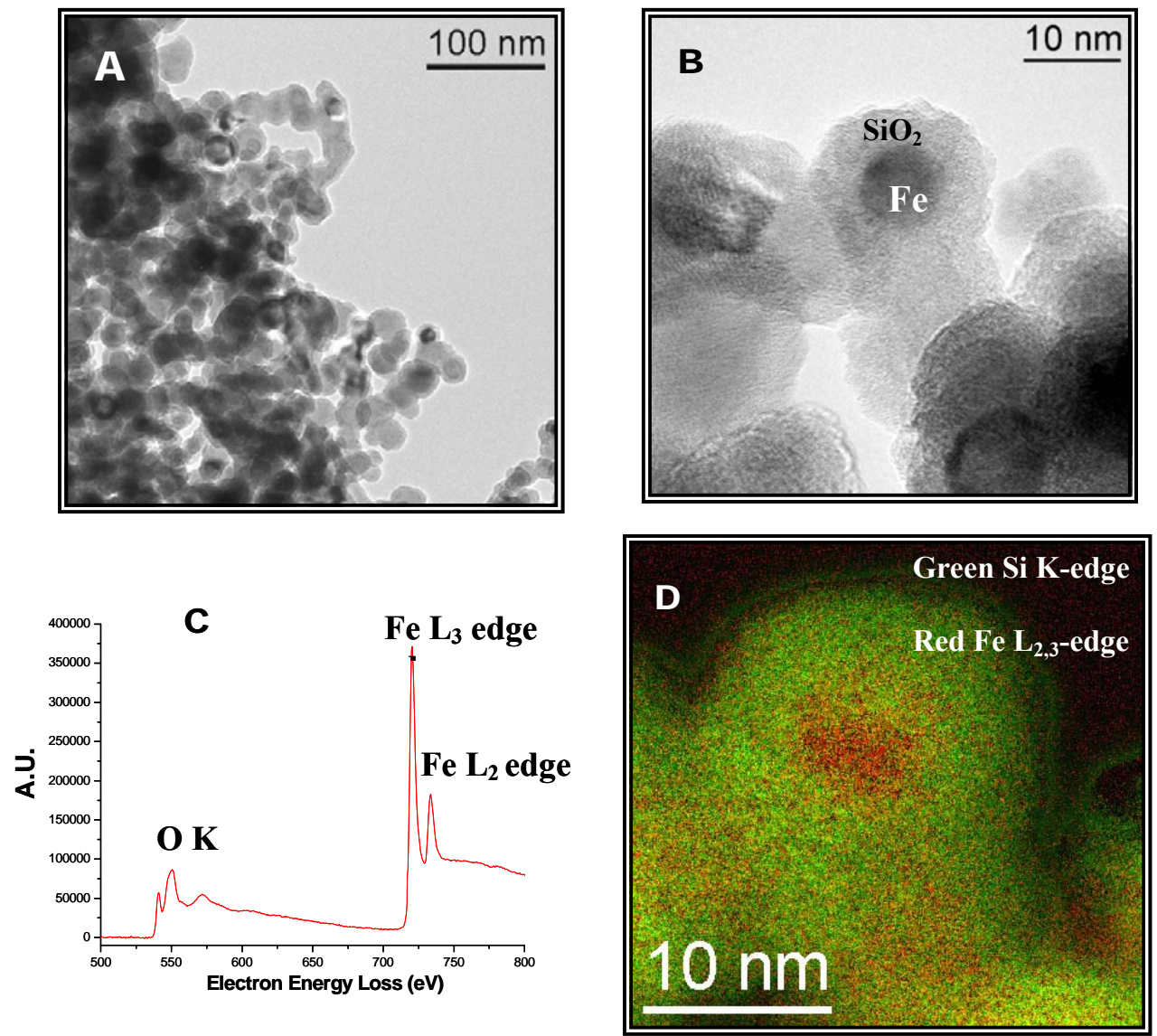

Figure 3.11 Transmission Electron micrographs of the silica-coated iron nanoparticles obtained from Y zeolite powder. (C) Electron Energy Loss Spectra (EELS) of the same particle. (D) EFTEM shows an iron-rich core and a silica-rich shell.

On the other hand, when oxidized metallic Silicon was used as the silica source, the presence of the silica coating is again observed for all the particles. This was identified by examining the low energy EELS plasmon region, where a 
maximum at $22.4 \mathrm{eV}$ characteristic of $\mathrm{SiO}_{2}$ was observed. Two types of atomic planes have been observed, corresponding to $\mathrm{Fe}_{2} \mathrm{O}_{3}$ (hematite) and to metallic iron. Filtering separately the spots corresponding to each structure on the Fourier space, and applying the inverse FFT algorithm to each filter, a very thin $(2.3 \mathrm{~nm})$ layer of iron oxide (hematite) surrounding the metallic Fe core can be observed (Fig. 3.12).

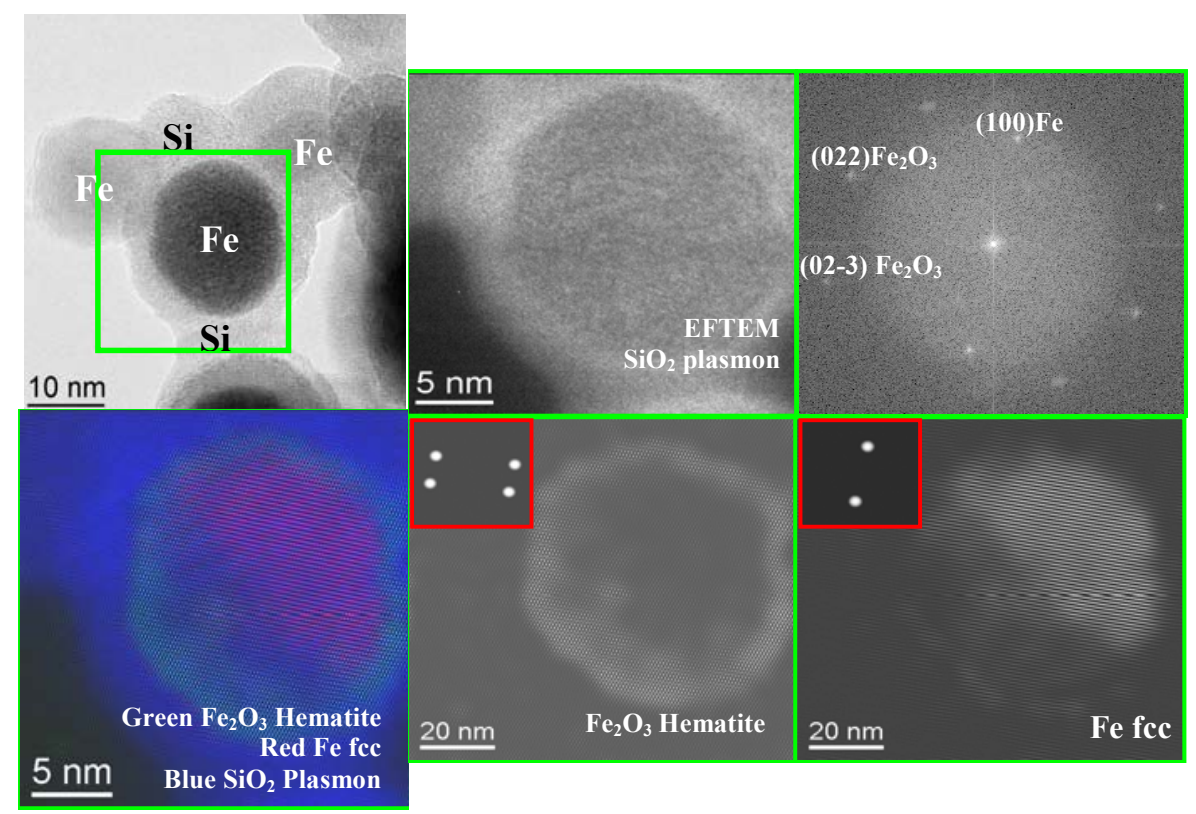

Fig. 3.12 EFTEM and HRTEM patterns for the $\mathrm{Fe} @ \mathrm{SiO}_{2}$ particles obtained using metallic silicon as the silica precursor. Blue: $\mathrm{SiO}_{2}$ plasmon. Red: Fe fcc HRTEM structure. Green: $\mathrm{Fe}_{2} \mathrm{O}_{3}$ hematite HRTEM structure. After obtaining the electron diffractogram of the sample, a Fast Fourier Transform (FFT) is applied the result and the obtained spots can be compared to the theoretical values of the distances between atomic planes. That way the crystallographic phase is obtained, and metallic iron can be distinguished from its oxides.

This could not be seen for all the particles, since most of them crystallize in a $b c c$ iron phase. EELS spectra clearly proved that the particle cores were very rich 
in iron, whereas the presence of oxygen was much smaller, due only to the thin pasivation layer of iron oxide and the $\mathrm{SiO}_{2}$ coating.

Especially for the first preparation, (using Y-zeolite as the silica source) the particle size distribution is narrow, with the materials produced being rather homogenous in size and shape. The presence of silica bridges among some of the particles can also be observed (Fig. 3.13).
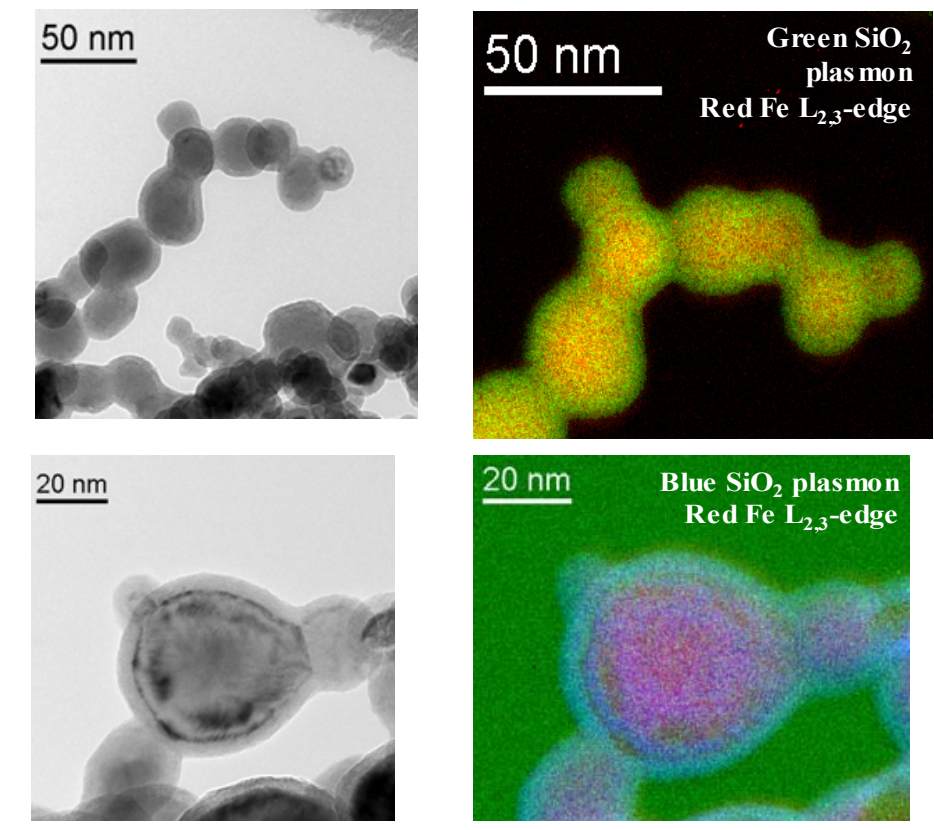

Fig. 3.13 EFTEM and HRTEM patterns for the Fe@SiO2 particles obtained using metallic silicon as the silica precursor. The presence of silicon bridges aggregates the particles.

\subsubsection{X-Ray Diffraction}

XRD results revealed the typical diffraction pattern of $\alpha$-Fe (Fig. 3.14). The characteristic diffraction peaks for quartz or $\mathrm{Y}$ zeolite at the low angle region did not appear, probably due to the fact that only amorphous silica is coating the iron cores, something that correlates with TEM results. Using the Scherrer equation and 
fitting the characteristic peak at $2 \theta=44.5 \mathrm{deg}$ to a PseudoVoigt function, the average particle size of the iron cores of the bulk sample was $56 \mathrm{~nm}$. However, TEM micrographs show smaller particle sizes around 10-30 nm. Elementary analysis (ICP-AES) gave an average amount of $12.6 \pm 0.5 \mathrm{wt} \%$ of Silica per total mass of sample.

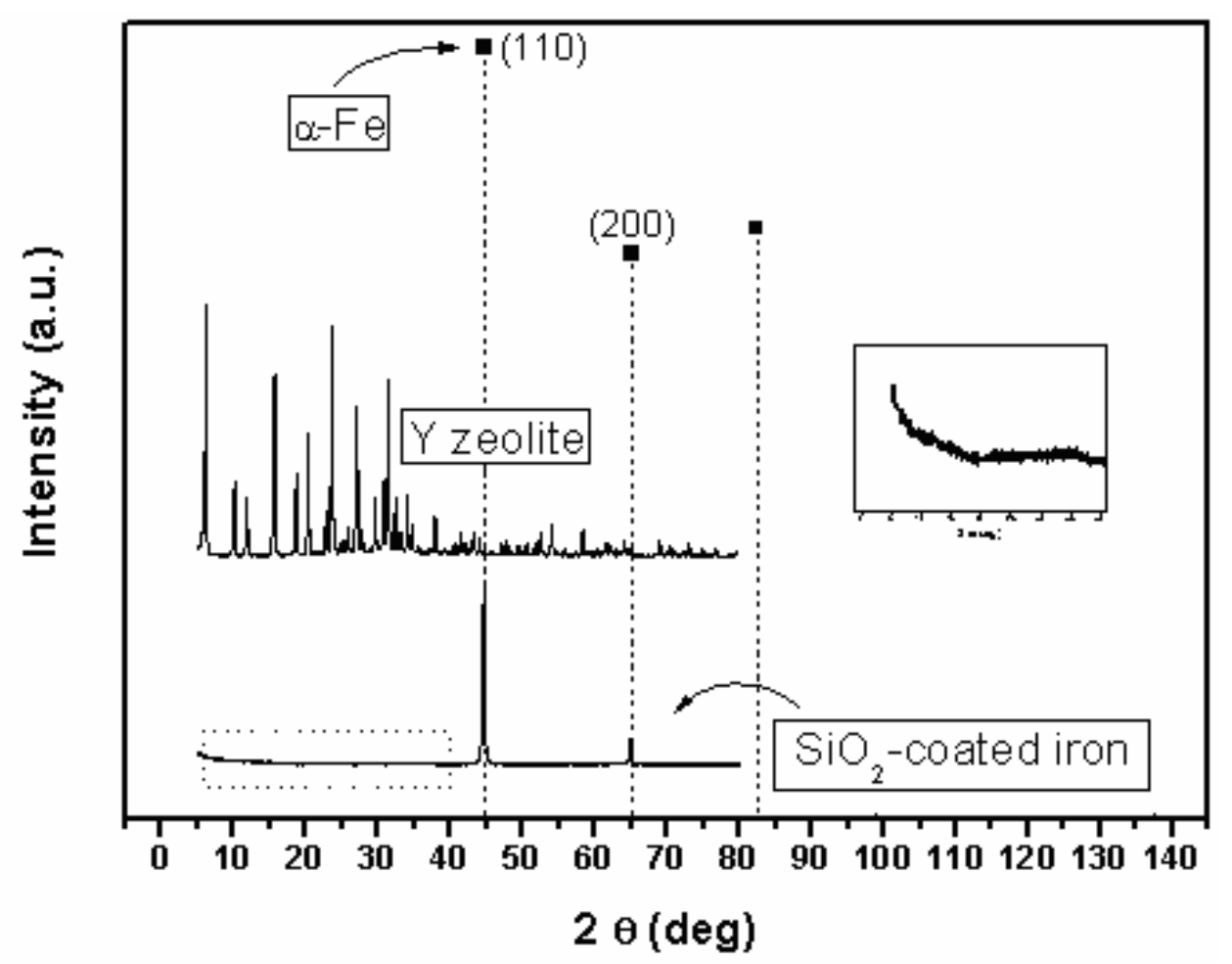

Fig. 3.14 XRD diffractograms for the Y-zeolite, $\alpha$-Fe and silica-coated iron nanoparticles patterns. The obtained sample presents peaks that correspond to the metallic iron phase. The crystalline structure of zeolite is not preserved after preparation. Inset: the low angle region magnification shows a slight hump that could correspond to the presence of amorphous silicon encapsulating the particles. 


\subsubsection{BET surface area}

The $\mathrm{N}_{2}$ adsorption/desorption isotherms for the silica-coated iron nanoparticles are also displayed in Fig. 3.15. Pore size distributions were calculated using the adsorption branch of the $\mathrm{N}_{2}$ adsorption/desorption isotherm and the Barret-JoynerHalenda (BJH) method. It is important to point out that the BJH method has been reported to underestimate the pore size. The micropore volume of the sample was almost 0 , as calculated from the $t$-plot method, which is consistent with a particle coating consisting of mesoporous silica. The mesopore size was $5.9 \mathrm{~nm}$ and the obtained BET surface area was $30.61 \mathrm{~m}^{2} / \mathrm{g}$.

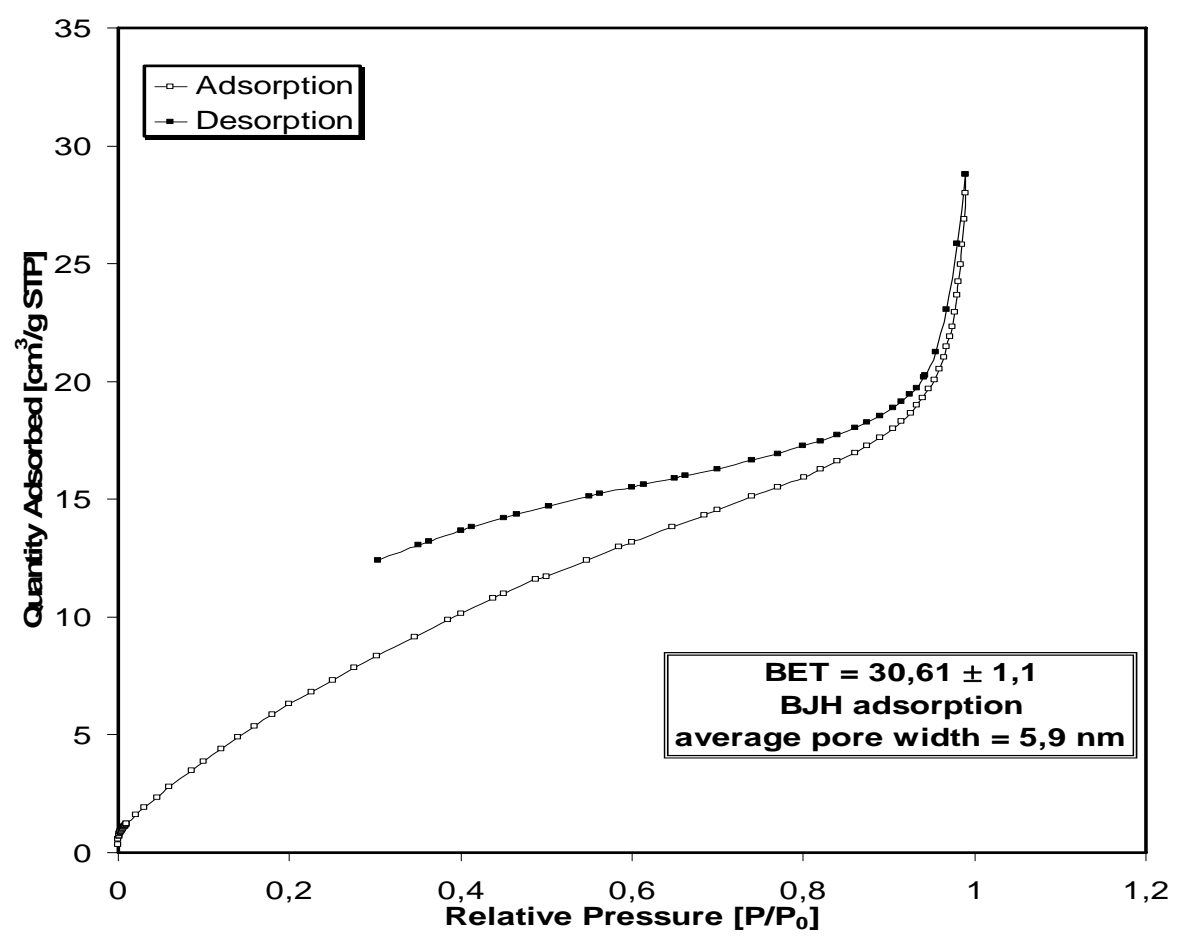

Fig. $3.15 \mathrm{BET}$ adsorption and desorption isotherms for the $\mathrm{Fe} @ \mathrm{SiO}_{2}$ particles obtained by discharge-arc. 


\subsubsection{XPS studies}

XPS analysis provides information about the chemical coposition of the surface of our material. The spectra prove the presence of silica insulating the particles: a Si 2 s peak $(153 \mathrm{eV})$ can be clearly observed; it nearly disappears after etching the outer coating (Fig. 3.16), whereas the Fe $2 p$ peaks increase significantly. Moreover, more detailed information on the existence and nature of the iron oxides present at the particles surfaces can be attained by careful inspection of the XPS spectrum shown in Figure 3.17. This spectrum is characterized by the existence of two main peaks at $710.3 \mathrm{eV}$ and other at $723.8 \mathrm{eV}$ which respectively correspond to $\mathrm{Fe}^{+3} 2 \mathrm{p}_{3 / 2}\left(\mathrm{~L}_{3}\right)$ and $2 \mathrm{p}_{1 / 2}\left(\mathrm{~L}_{2}\right)$ edges, as previously observed [Fuji1999]. It is clearly noticed the existence of a satellite peak at an energy binding of $718.8 \mathrm{eV}$, which is the fingerprint of iron oxide $\left(\mathrm{Fe}^{+3}\right)$ [Mossbauer1990]. It is also perceptible the existence of very small peaks at $\sim 706.9$ $\mathrm{eV}$ and $719.8 \mathrm{eV}$ which can be associated with the presence of some very small residual metallic iron phases at the surface.

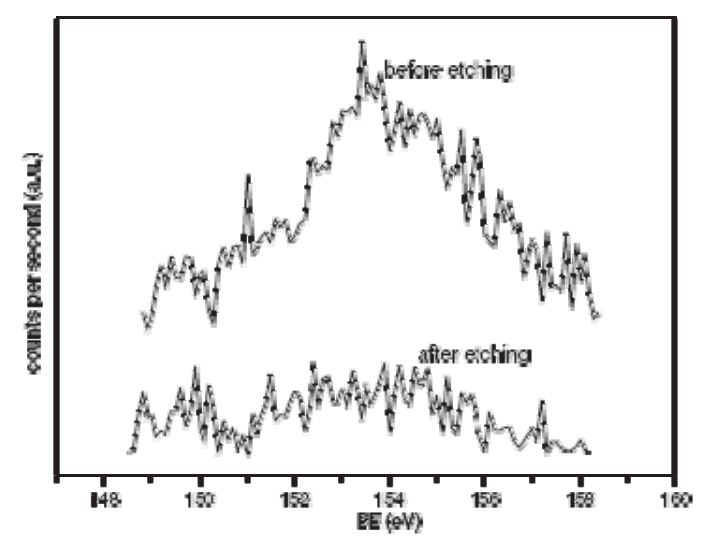

Fig. 3.16: XP spectra of iron encapsulated in silica corresponding to silicon before and after Argon etching ( $5 \mathrm{~nm}$ depth). Silicon peak disappears after the effect of the ions etching, which remove the silicon encapsulation of the particles. 

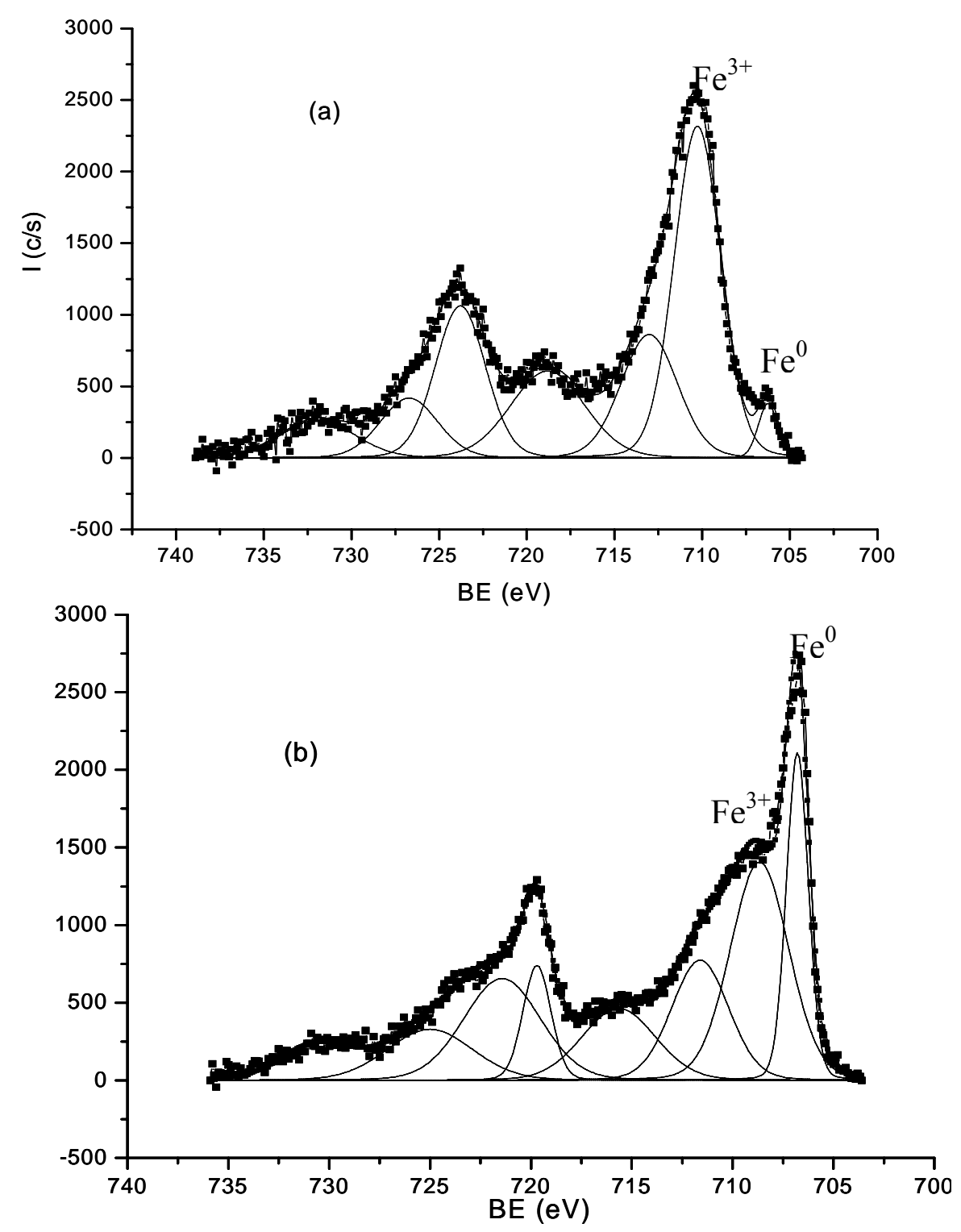

Fig. 3.17 XPS spectra of iron encapsulated in silica, a) before and b) after argon etching (approximately $5 \mathrm{~nm}$ depth), probing the iron core of the encapsulated nanoparticles. The coating of iron oxide is etched, therefore decreasing the $\mathrm{Fe}^{+3} 2 \mathrm{p}_{3 / 2}$ edge and increasing the $\mathrm{Fe}^{0} 2 \mathrm{p}$ edge of the particle's core. 
In-situ low-energy Ar etching was performed. In this etching process a depth profiling of around $5 \mathrm{~nm}$ is estimated. As a consequence, after the etching process the particles core is expected to be probed. The results are shown in Fig 3.17 in which a strong enhancement of the $\mathrm{L}_{3}$ and $\mathrm{L}_{2}$ metallic $\mathrm{Fe}^{0}$ edges can be observed. These correspond to the typical spectra of full metallic iron. This is an indication that the particles cores are iron as shown by HRTEM results. The relevance of combining both experimental techniques has to be underlined, as HRTEM probes just on a few manometers region, while XPS gives information about the electronic states of the elements, scanned on larger areas of the samples.

\subsection{Magnetite encapsulated in silica. $\mathrm{Fe}_{3} \mathrm{O}_{4} @ \mathrm{SiO}_{2}$}

By a combination of the co-precipitation method and the sol-gel technique, small magnetite cores have been encapsulated in silica to obtain particles of a size in the range of $80 \mathrm{~nm}$. Iron-based magnetic particles less than about $25 \mathrm{~nm}$ exhibit superparamagnetism at room temperature [Lee1996]. Also, bare superparamagnetic iron oxide nanoparticles are considered to be biodegradable. Iron would be reused or recycled by cells using normal biochemical pathways for iron metabolism [Bulte2004, Saebo2004]. This is supported by the pharmacokinetic data published for commercial iron oxide-based contrast agents (e.g., Feridex IV ${ }^{\mathrm{TM}}$, from Advanced Magnetics, Inc.), showing that at the usual doses a significant fraction of the iron in superparamagnetic iron oxide nanoparticles can enter the normal iron metabolism cycle, becoming part of the body iron pool, while unabsorbed iron can be excreted (mainly via urine). On the other hand, the size of the obtained superparamagnetic nanoparticles $(\sim 80 \mathrm{~nm})$ is smaller than the spleen filtration cutoff (200 nm) [Fahmy2005], meaning that they are not likely to accumulate in the spleen; it is therefore reasonable to expect that a significant fraction of these nanoparticles will be excreted via urine and bile. In any case, further studies are 
needed to determine the extent of iron assimilation in silica-coated iron cores, and the final fate of the silica coating itself.

Even though limited amounts of iron can be assimilated, it is also true that an excess of exposed iron would present toxicity problems, and therefore the presence of bare (i.e., non-coated) iron must be minimized. This requisite is fulfilled in these particles, where the presence of iron at the surface was less than 0.6 atom $\%$, (the limit of detection of XPS analysis under the used conditions).

\subsubsection{X-ray diffraction}

XRD analysis carried out on both silica-coated and uncoated magnetic cores showed the presence of the characteristic diffraction peaks of magnetite/maghemite not only for the iron oxide nanoparticles but also for the silica-coated iron oxide nanoparticles (Fig. 3.18).

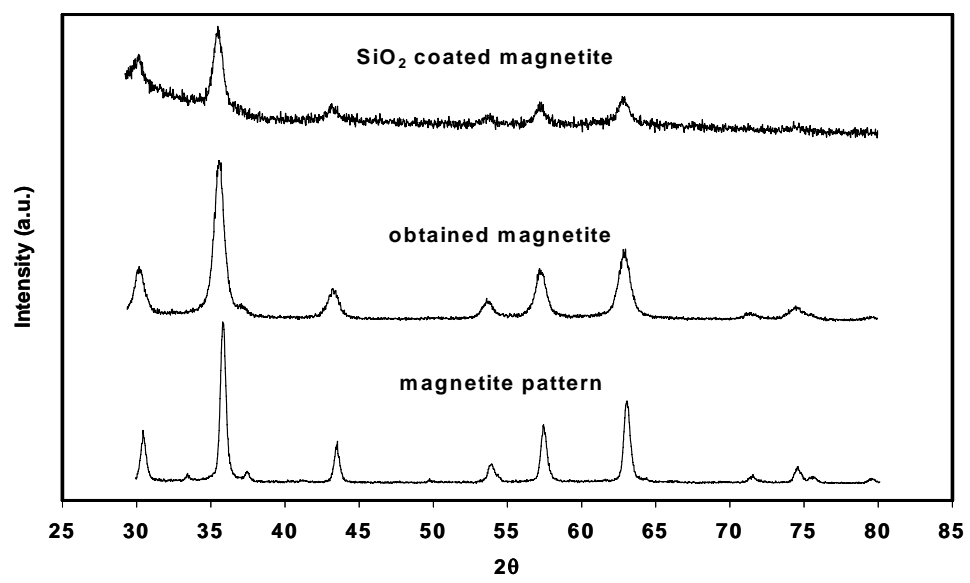

Figure 3.18 X-ray diffractograms of the magnetite pattern, the obtained magnetite nanoparticles before sol-gel encapsulation treatment and the silica-coated magnetite nanocomposites. The final product preserves the peaks of magnetite, with a slight decrease in intensity due to the presence of the silica coating. 
However, XRD analysis does not allow discrimination between magnetite $\left(\mathrm{Fe}_{3} \mathrm{O}_{4}\right)$ and maghemite $\left(\gamma-\mathrm{Fe}_{2} \mathrm{O}_{3}\right)$ because both have similar crystallographic structure and their diffraction patterns overlap. Thus, further characterization (HRTEM, EELS and XPS) was carried out, as shown below. The intensity of the XRD peaks decreased after coating the particles with silica due to the effect of the amorphous silica shell. No other phases (i.e., hematite) were found. Using the Scherrer equation (see section 2.1.6) and fitting the characteristic peak at $2 \theta=35.9$ deg ([311] diffraction plane) to a PseudoVoigt function, the average particle size of the iron-oxide cores of the sample was estimated as $17 \mathrm{~nm}$.

\subsection{2 $\mathrm{N}_{2}$ adsorption/desorption isotherms}

The $\mathrm{N}_{2}$ adsorption/desorption data obtained with the silica-coated nanoparticles approached a Type II isotherm, characteristic of macroporous materials with surface heterogeneity. However, the isotherm also showed a microporous pattern in the region of low relative pressures $\left(<0.05 \mathrm{P} / \mathrm{P}_{0}\right)$. The micropore volume of the sample was $0.005 \mathrm{~cm}^{3} / \mathrm{g}$, which is consistent with a small contribution of the microporous silica to the macroporosity of the composite. The macroporous average pore width was $32.5 \mathrm{~nm}$ and the BET surface area was 48.7 $\mathrm{m}^{2} / \mathrm{g}$ of which $38.2 \mathrm{~m}^{2} / \mathrm{g}$ corresponding to the external surface area. The macroporosity can be attributed to the spaces between particles, which is in agreement with the results obtained using HRTEM, which are discussed next. The microporous average pore width was $1.5 \mathrm{~nm}$ from the Horvath-Kawazoe analysis of the $\mathrm{N}_{2}$ desorption data. 


\subsubsection{Transmission Electron Microscopy}

TEM micrographs showed roughly spherical silica particles with a metallic core (Figure 3.19). The mean nanoparticle size was approximately $80 \mathrm{~nm}$ for the composite material, while the average size for the magnetic cores varied between 6 and $20 \mathrm{~nm}$.

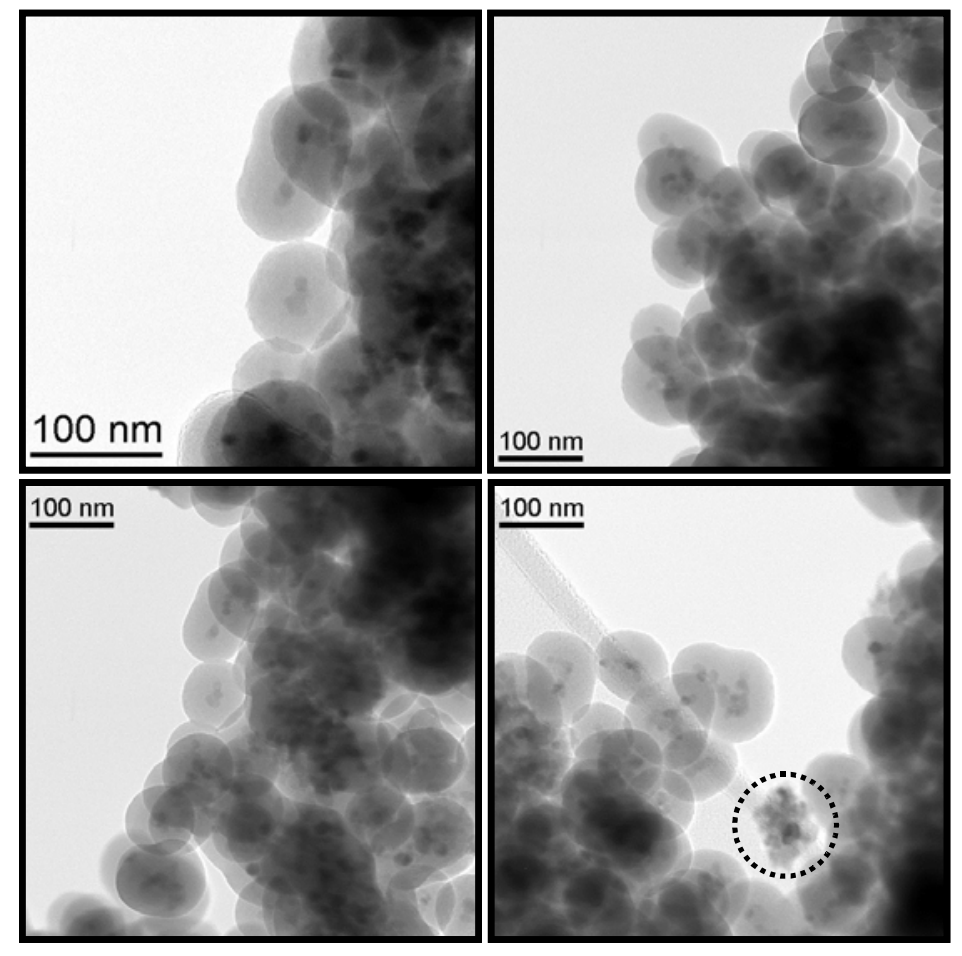

Fig. 3.19 TEM photographs for the silica coated iron oxide nanoparticles. Some free iron oxide (dot-line) can be seen in the bottom right image. The perfect encapsulation of the iron oxide cores, the homogeneity of the sample and the narrow size distribution can be observed from the images,.

On the other hand, EFTEM micrographs (Fig. 3.20) clearly showed that the iron oxide nanoparticles were almost always coated with an amorphous silica shell, 
and free magnetite seldom occurred in the samples analyzed. According to the results presented so far, it seems appropriate to describe the obtained product as a relatively homogeneous collection of nanoparticles with an average size of $80 \mathrm{~nm}$, consisting of an iron oxide core encapsulated in a microporous silica shell.
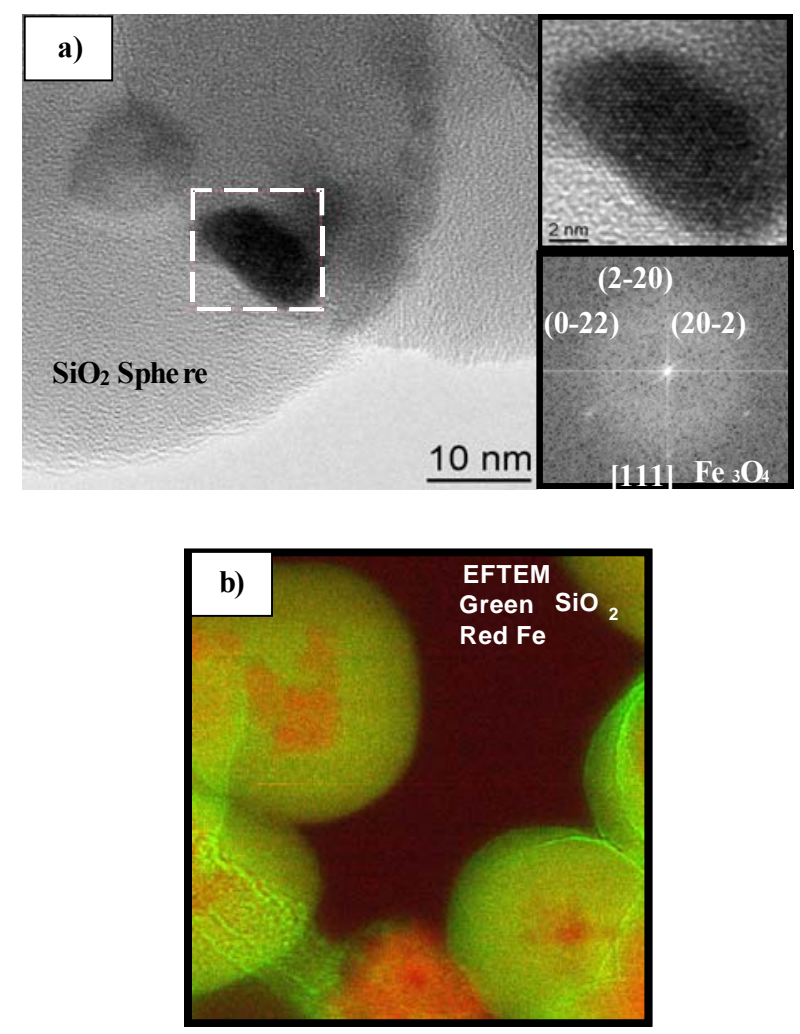

Fig. 3.20 a) HRTEM micrograph of the $\mathrm{Fe}_{3} \mathrm{O}_{4}$ magnetite nanoparticles encapsulated within the silica shell and structural analysis by means of the power spectrum. b) EFTEM colour map showing the Iron (red) atomic distribution (in this case forming the $\mathrm{Fe}_{3} \mathrm{O}_{4}$ nanoparticles), and the silicon (green) distribution, as part of the shells.

Concerning the nature of the iron oxide cores, HRTEM analysis and EELS (Fig. 3.21) revealed patterns consistent with the presence of magnetite $\left(\mathrm{Fe}_{3} \mathrm{O}_{4}\right)$. Analysing the ELNES spectrum for the iron $\mathrm{L}_{2}$ and $\mathrm{L}_{3}$ peaks it is possible to calculate the relative proportions of iron oxidation states in the mixture 
[Colliex1991, Van Aken1998] and to distinguish between magnetite and maghemite. Calculating the ratio of the integrated areas of the peak a ratio $\mathrm{I}\left(\mathrm{L}_{3}\right) / \mathrm{I}\left(\mathrm{L}_{2}\right) \approx 2.82$ was obtained, indicating a high content of $\mathrm{Fe}^{2+}(73.25 \%)$ compared to $\mathrm{Fe}^{3+}(26.5 \%)$. This again indicates that maghemite is mostly absent from the iron oxide phase.

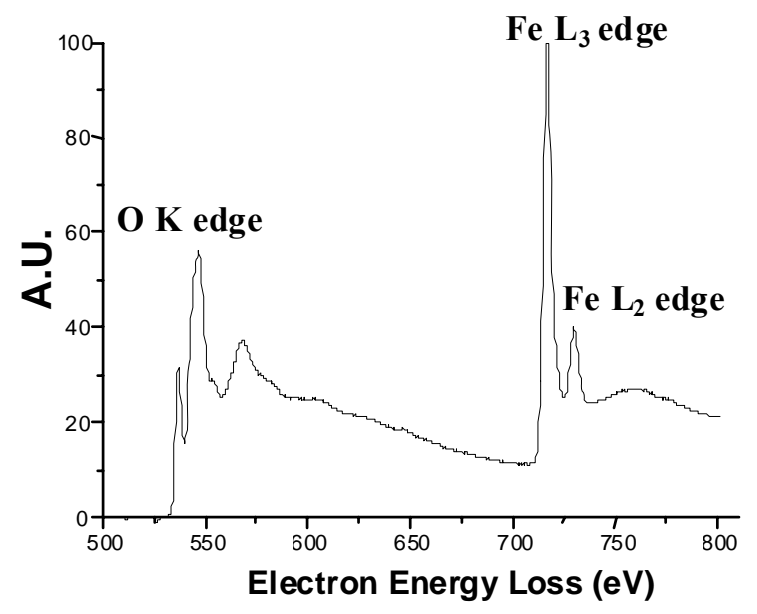

Fig. 3.21 EELS Spectra of the $\mathrm{Fe}_{3} \mathrm{O}_{4} @ \mathrm{SiO}_{2}$ particles. From the quantitative relationship between iron and oxygen edges the presence of magnetite is confirmed.

\subsubsection{DLS Estimation of Size}

The particle-size distribution for the silica-coated nanoparticles at $\mathrm{pH} 7.3$ measured by DLS yielded an average value of $98 \pm 15 \mathrm{~nm}$ in broad agreement with the TEM results. These results suggest that, at the $\mathrm{pH}$ of the blood, the silica shell helps to prevent particle agglomeration, giving a stable suspension. Following attachment of the antibody, the particle-size distribution for the antibodyconjugated nanoparticles was determined again by DLS, yielding an average size

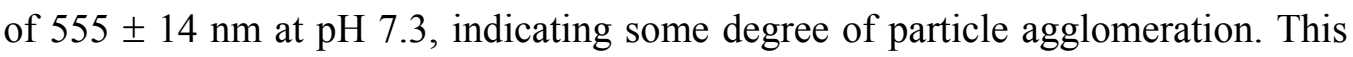
agglomeration disappeared by applying a rehydration protocol to the particle 
suspension, bringing again the average particle sizes to values slightly below 100 $\mathrm{nm}$.

\subsubsection{Magnetic measurements}

The magnetic measurements carried out at different temperatures for the silica coated iron oxide nanoparticles are shown in Figure 3.19. As could be expected from the above discussion, the silica-coated iron oxide nanoparticles show a behaviour close to superparamagnetism, with a magnetic moment of $10.3 \mathrm{emu} / \mathrm{g}$ at 4 Tesla and a coercivity of $15.7 \mathrm{Oe}$ at $310 \mathrm{~K}$. The low coercive field can be related to the remanence of the magnetometer. It must be noted that magnetic moment values in this case are given per unit of total mass $(\mathrm{emu} / \mathrm{g})$, that is, considering the total weight of both silica and iron in the nanoparticles, therefore yielding overall magnetic moments lower than those measured for commercial superparamagnetic iron oxide preparations (e.g., Feridex IV ${ }^{\mathrm{TM}}, 68 \mathrm{emu} / \mathrm{g} \mathrm{Fe}$ ). [Bomatí2005] When the magnetite cores synthesized in this work were measured separately at 3 Tesla, they yielded a magnetic moment as high as $68.7 \mathrm{emu} / \mathrm{g}$. This is lower than the magnetic moments measured for pure magnetite samples $(92 \mathrm{emu} / \mathrm{g})$ due to the increased disorder at the particle surfaces as their size is decreased [Huang2004]. If 68.7 $\mathrm{emu} / \mathrm{g}$ is considered as the magnetic moment corresponding to the bare nanoparticle cores, the magnetite content estimated for the composite would be approximately $15 \mathrm{wt} . \%$, which is similar to the values expected from the relative amounts of magnetite and silica used in the sol-gel procedure $(12.7 \mathrm{wt}$. \% of magnetite).

Above $150 \mathrm{~K}$ the coercivity values are comparable to those of the remanent field of the coils in the magnetometer, i.e. the coercivity of our samples can be assumed to be practically zero. When magnetisation versus field/temperature is plotted for each measurement (Figure 3.22 a) above $150 \mathrm{~K}$ there is a clear 
overlapping of the graphs, again characteristic of the superparamagnetic behaviour of the sample.
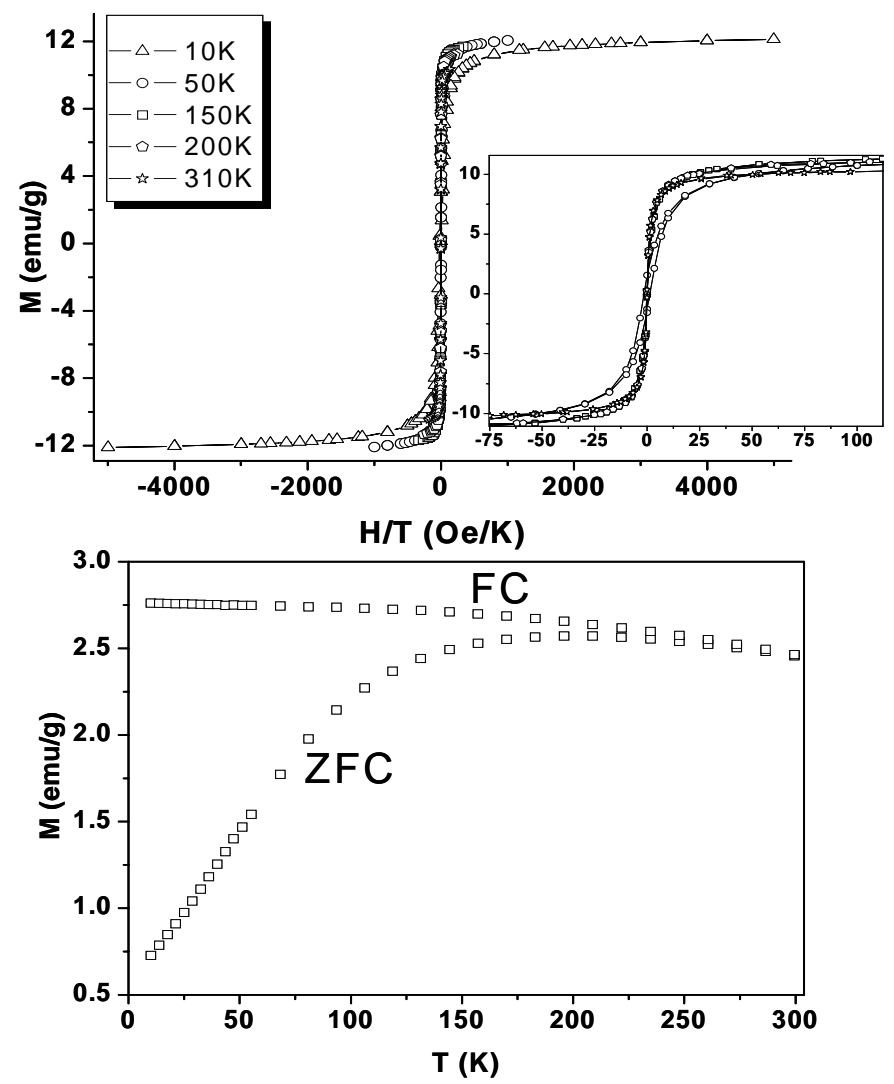

Fig. 3.22 a) Magnetisation Vs Field/Temperature for the obtained silica coated iron oxide nanoparticles at different temperatures. Above $150 \mathrm{~K}$ graphs are superimposed, collapsing them into one. Inset: zoom in to show the coercivity field; b) ZFC and FC magnetisation curves of the silica-coated magnetite nanoparticles.

The temperature dependence of the magnetisation was also measured. By means of the Zero-field-cooling (ZFC) and field cooling (FC) analysis the blocking temperature of the sample (maximum of the ZFC curve) can be obtained, above which the particles are superparamagnetic (Figure 3.22 b). 
Below $150 \mathrm{~K}$ all the particles are blocked, their magnetic moments remain aligned along the direction of the applied magnetic field, displaying ferromagnetic behaviour. The presence of blocked particles below $150 \mathrm{~K}$ is in strong agreement with the magnetisation measurements at different temperatures. These magnetic measurements indicate superparamagnetic behaviour of the composite (magnetitesilica) nanoparticles at room temperature, thus making them suitable for their use in potential applications as MRI contrast agents.

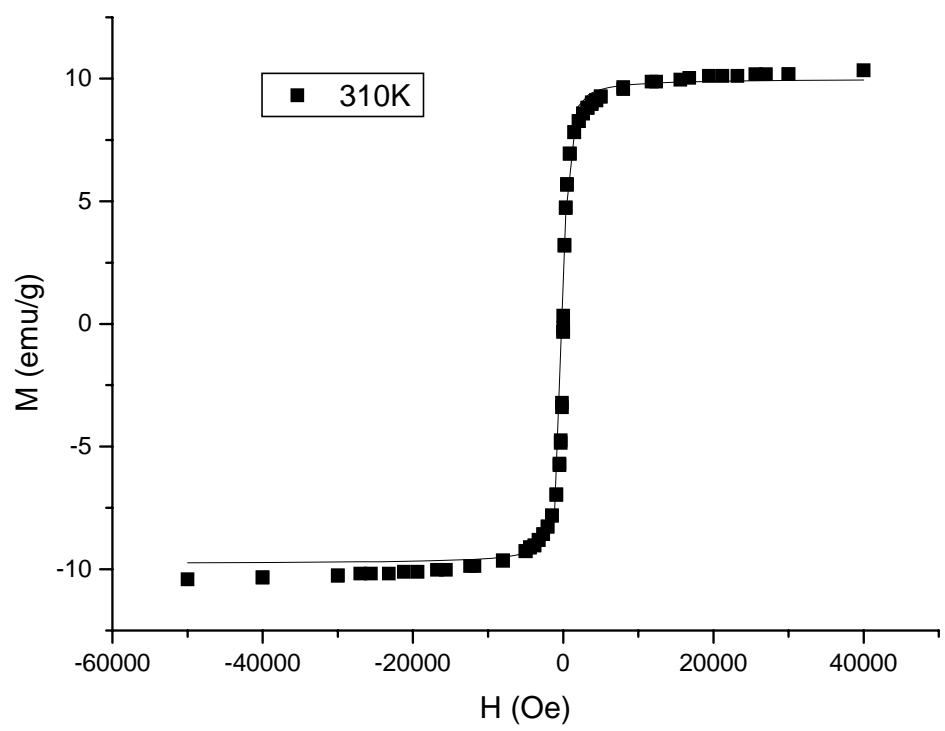

Figure 3.23 Langevin fit to the experimental isotherm for the $\mathrm{Fe}_{3} \mathrm{O}_{4} @ \mathrm{SiO}_{2}$ particles. The high value for $\mathrm{R}^{2}=0.99732$, indicated that experimental data accurately follow Langevin law. Form the obtained fit equation the diameter of the particles can be calculated. A value of $15 \mathrm{~nm}$ of diameter was estimated with this method.

Particles above $150 \mathrm{~K}$ are in the superparamagnetic state; therefore they are expected to follow the Langevin law. By fitting the magnetisation isotherm to the Langevin function (Fig. 3.23), the mean size of the particles can be estimated. Assuming a spherical shape for the particles, a size of $15 \mathrm{~nm}$ was obtained, which is comparable to the sizes measured by TEM. This size suggests the presence of 
single-domain magnetite nanoparticles, in agreement with all the previous measurements.

\subsection{Magnetite embedded in a zeolite matrix. $\mathrm{Fe}_{3} \mathrm{O}_{4} @ \mathrm{ZY}$}

The mechanical milling of magnetite and Y-zeolite powders in a ball mill yielded a composite that exhibits magnetic properties and a high surface area. This material was also characterised morphologically and magnetically.

\subsubsection{SEM and TEM}

SEM photographs after milling (Fig. 3.24) showed a bulk material with a particle size distribution from $20 \mathrm{~nm}$ to $1.2 \mu \mathrm{m}$. However, the range of particle sizes measured by PCS for this sample (volume average) extended from 20 to 600 $\mathrm{nm}$, probably because the larger particles settle during DLS measurements. Although there are isolated nanosized particles (demonstrated by HRTEM), agglomerations up to $1.2 \mu \mathrm{m}$ were observed.
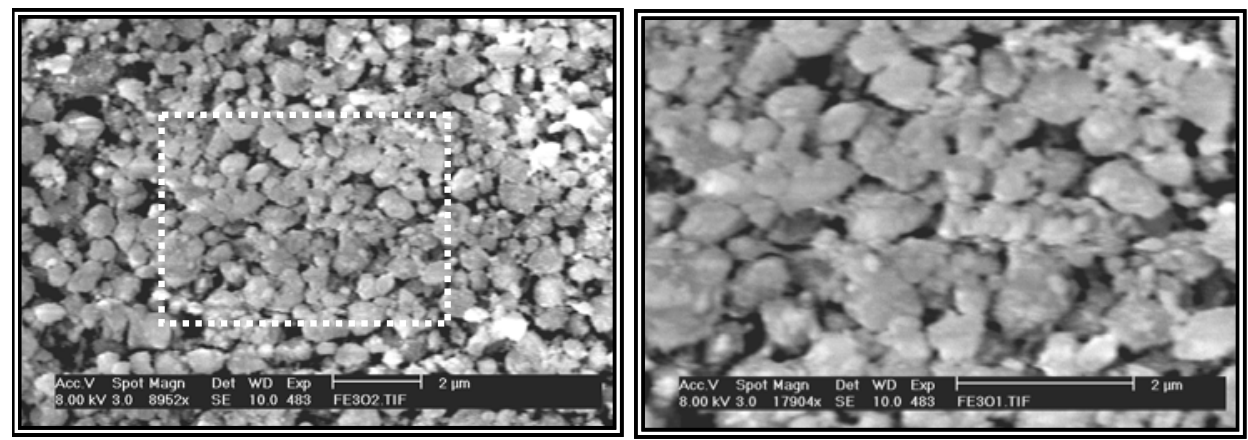

Figure 3.24 Scanning Electron Micrograph of the bulk composite material. The right side is a magnification of the picture on the left.

Even so, the largest dimension of these measured crystals is still considerable smaller than the capillary diameter of the human body ( $8 \mu \mathrm{m}$ [Ritter2004]) which 
makes their size suitable for intravenous injection in drug-delivery applications. In addition, the hydrophilic zeolite shell would provide the vector with the required biocompatibility needed to minimize the reticuloendothelial system response.

As expected, the HRTEM micrographs revealed the presence of magnetite in the composite material. As an example, Figure 3.25.a shows a region in which several iron oxide nanoparticles are visible. The Power Spectrum (Fig. 3.25.c), obtained after applying the Fast Fourier Transform (FFT) algorithm to the HRTEM image selection (Fig. 3.25.b), allowed the precise identification of the nature of these particles by showing the inverse spinel structure characteristic of magnetite. A further confirmation of the encapsulation of the magnetite nanoparticles within a zeolitic shell was provided by EFTEM (Figure 3.26). In Figure 3.26.b, the red pixels correspond to the presence of iron atoms (in this case forming $\mathrm{Fe}_{3} \mathrm{O}_{4}$ magnetite oxide nanoparticles) while green pixels correspond to the presence of silicon atoms (in this case as part of Y-zeolite aluminosilicate). There are no visible free magnetite nanoparticles and it can be observed that all the magnetite nanoparticles are surrounded by a thin $(4 \mathrm{~nm})$ zeolite layer. Those results are consistent with the observations discussed concerning the XRD patterns of the particles attached to the magnetic stirrer, where the presence of zeolite was evident, and with the XPS results regarding the $\mathrm{Si} / \mathrm{Fe}$ ratios of the surface material, that also indicated that magnetite particles were coated by a zeolite layer. 

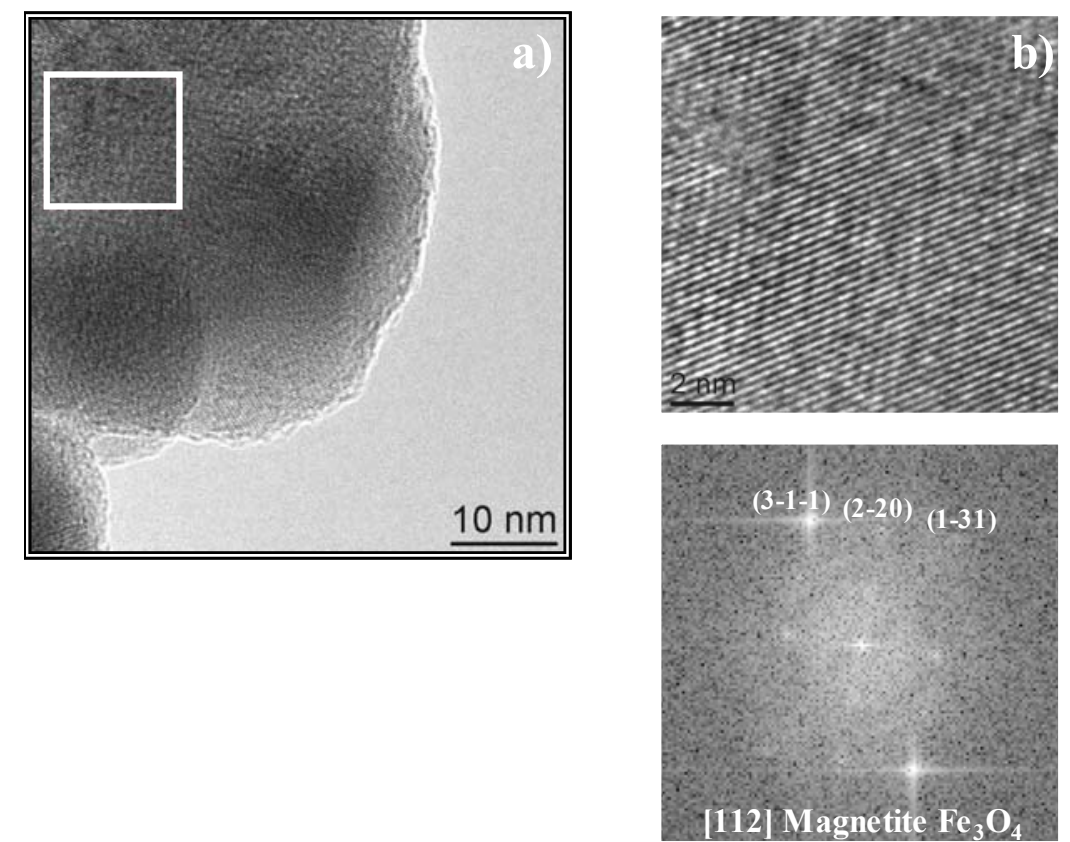

Fig. 3.25 a) HRTEM micrograph showing magnetite nanoparticles encapsulated within the zeolite crystals. b) HRTEM magnified detail extracted from the squared section in a). c) Power spectrum obtained after applying the FFT algorithm to the previous selection
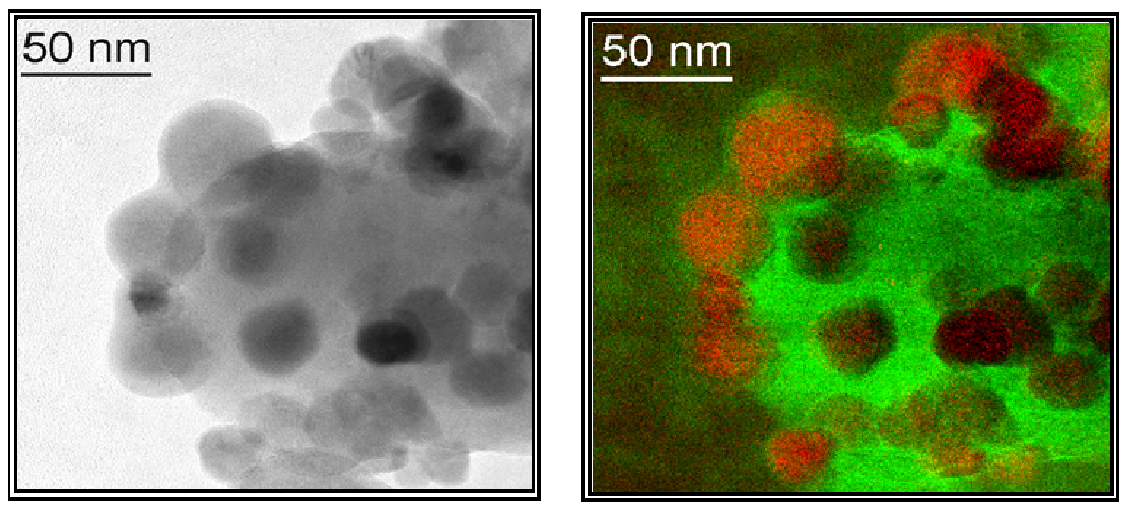

Fig. 3.26 a) Bright Field TEM (BFTEM) micrograph showing the magnetite particles encapsulated within the zeolite crystals. b) EFTEM colour map showing the iron (red) atomic distribution (forming the magnetite particles) and the silicon (green) distribution, as part of the zeolite crystals. 


\subsubsection{X-Ray Diffraction}

XRD patterns showed that the only reflections detected correspond to the zeolite and the magnetite phases; no other structures or alloys were present (Figure 3.27). Also, the figure shows that there is not appreciable distortion of the $Y$ zeolite/magnetite composite pattern compared to the parent zeolite, indicating the absence of changes in the cell dimensions of the zeolite crystals. These results are in contrast with the findings of Sorescu et al. [Sorescu2004], who reported a phase transformation of magnetite to hematite caused by high-energy ball milling. This evolution of phases is caused by the oxygen present during the ball milling in conditions of atmospheric pressure or low vacuum (0.1-5 torr). However, in this case, used high vacuum $\left(7.5 \cdot 10^{-5}\right.$ torr) was used, hence, although hematite was searched specifically, it was not detected in the XRD results, meaning that hematite was not formed under the milling conditions employed.

XRD was also used to assess the stability of the bond between the zeolite and magnetite in an aqueous environment. For that purpose, the composite material was immersed in distilled water and stirred overnight at room temperature. The particles strongly attached to the magnetic stirrer (the place where free magnetite would conceivable be preferentially collected) were analyzed using XRD, and the diffraction patterns were examined for an enrichment of the peaks corresponding to magnetite. The results are shown as the bottom curve in Figure 3.21, and it can be seen that they are undistinguishable from the curve obtained after mechanical activation. Hence, it can be concluded that, after prolonged exposure to water under stirring, magnetite is still firmly attached to the zeolitic matrix, and no significant segregation takes place. Those results are in agreement with the results obtained with XPS, FTIR, and HRTEM. 


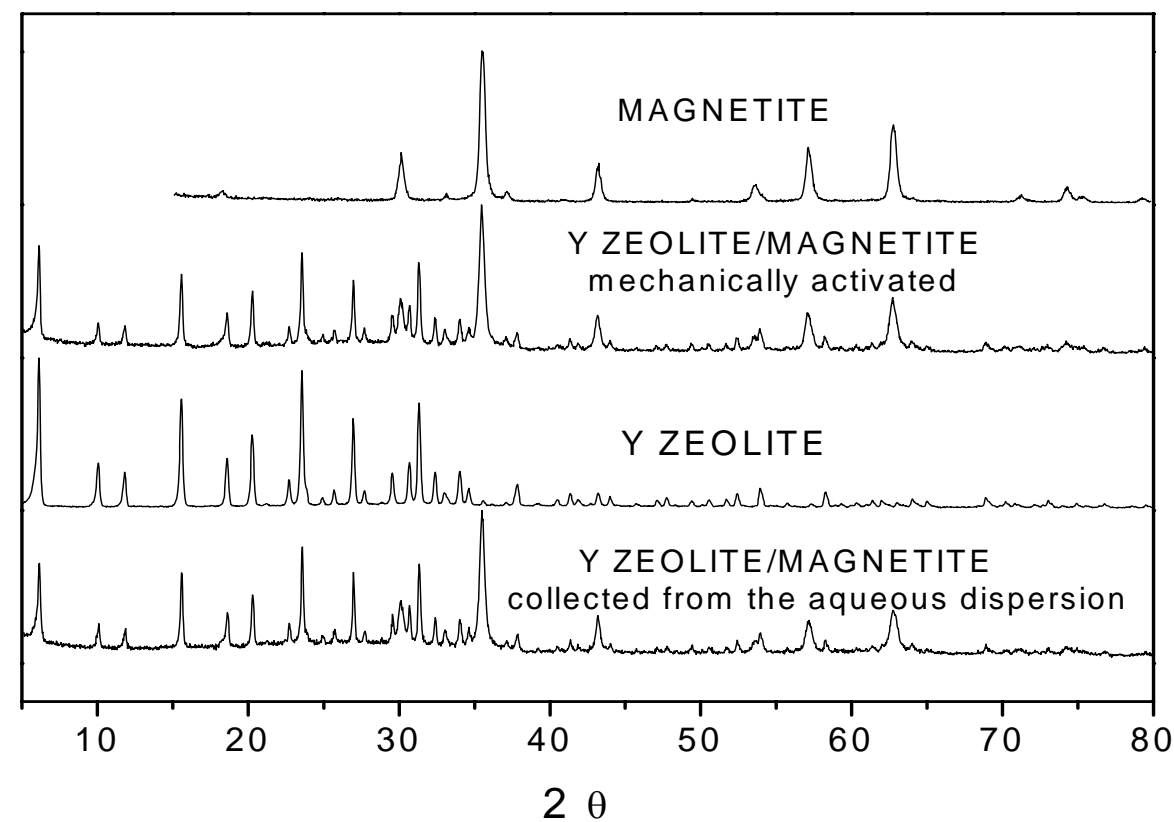

Fig. 3.27 X-Ray diffractograms of the parent materials (Y zeolite and magnetite) of the composite material obtained after milling and of the composite material after aqueous dispersion and magnetic separation.

\subsubsection{Magnetic Properties}

The SQUID measurements carried out at room temperature showed a saturation magnetisation at 2 Tesla as high as $16 \mathrm{emu} / \mathrm{g}$ with a coercitivity of 94.2 Oe (Fig. 3.28). This is a reliable indication of the existence of blocked magnetic particles at room temperature, in good agreement with the observation of aggregates of magnetic particles in our samples. Nevertheless it must also be noted that the lack of saturation of the magnetisation isotherm reflects a superparamagetic behavior resulting form the smallest isolated nanosized magnetic particles. An extrapolation of the SQUID measurements to high field values would give a value of approximately $17 \mathrm{emu} / \mathrm{g}$. It is important to point out that the magnetic moment values are given per unit of total mass $(\mathrm{emu} / \mathrm{g})$, that is, considering the total weight of both zeolite and magnetite in the composite 
material. Therefore, after taking into account the zeolite/magnetite ratio of 3:1 for the composite material a value of $\sim 68 \mathrm{emu} / \mathrm{g}$ would be obtained as the magnetite contribution, which is very close to the value for commercial magnetite (73.1 $\mathrm{emu} / \mathrm{g})$

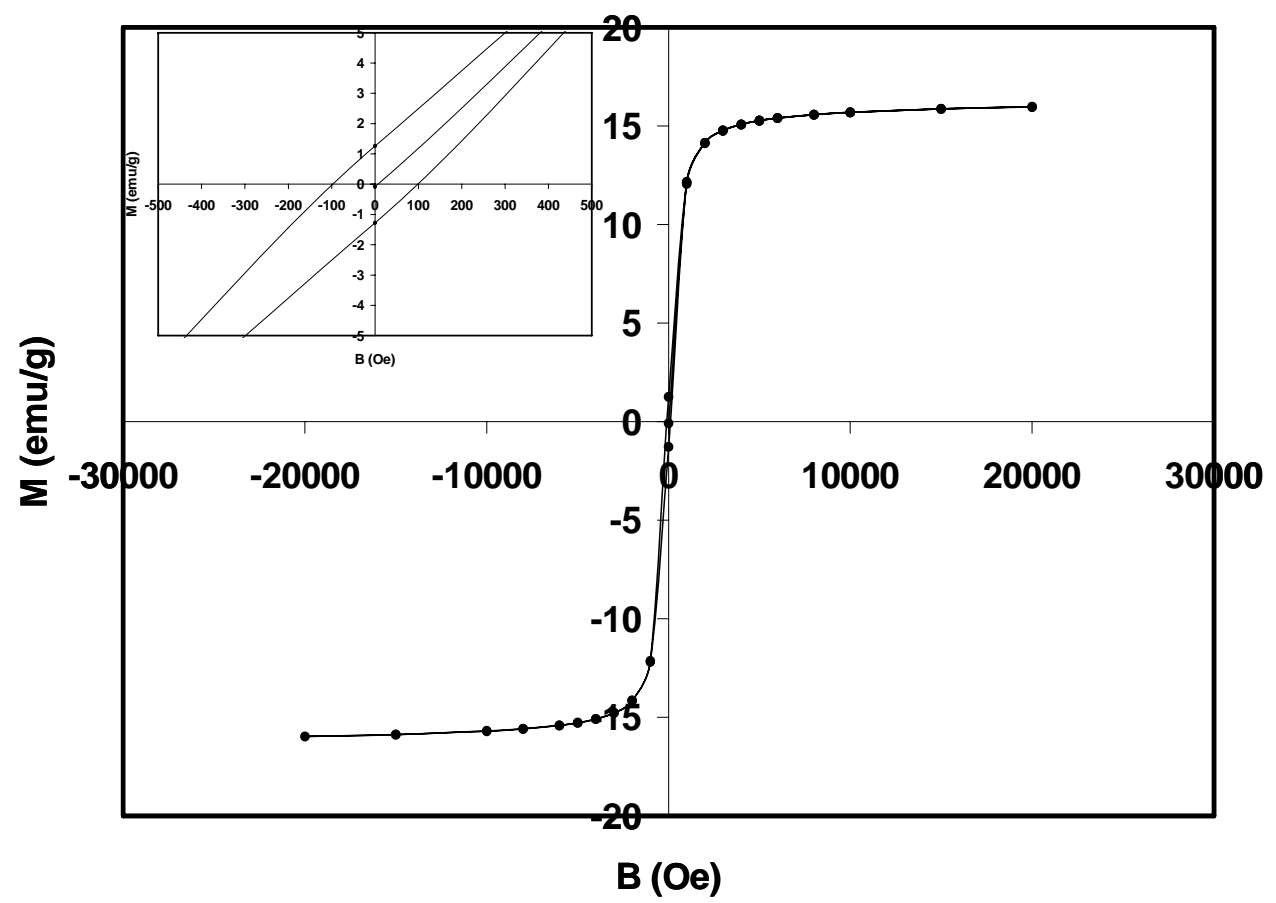

Figure $3.28300 \mathrm{~K}$ magnetisation isotherm for the composite material after $16 \mathrm{~h}$ of milling at room temperature. The inset details the hysteresis loop, showing a coercive field of 94.2 Oe.

The composite sample showed ferromagnetic behavior. The saturation magnetisation could be increased by means of optimizing the proportion of magnetite/zeolite in the starting material before milling, i.e., increasing the content of magnetite while avoiding the presence of free magnetite in the obtained composite. Comparing the particles obtained with the composite maghemite/Y zeolite prepared by the precipitation procedure by Oliveira et al. [Oliveira2004] with the same zeolite/iron oxide weight ratio of $3: 1$, a very similar saturation 
magnetisation can be observed (17 and $18 \mathrm{emu} / \mathrm{g}$, respectively), with a considerably higher BET surface area for the particles prepared in this work (442.9 versus $381 \mathrm{~m}^{2} / \mathrm{g}$ ). Thus similar total magnetisation values are obtained, using magnetite, in spite of the fact that this material has and intrinsically lower magnetic susceptibility than the material used in the work of Oliveira et al. (maghemite).

\subsubsection{BET surface area}

Figure 3.29 shows the characteristic Type I isotherm of microporous materials both for the initial zeolite $\mathrm{Y}$ and for the magnetite/zeolite composite. A strong decrease (from 900 to $486.1 \mathrm{~m}^{2} / \mathrm{g}$ ) of the surface area after calcination was observed for the parent zeolite, due to the condensation of surface silanol groups. After the inclusion of magnetite during milling the zeolite seems to retain its BET area (there is a perceptible decrease, from 486.1 to $442.9 \mathrm{~m}^{2}$ per gram of sample, but it must be taken into account that in the composite sample one quarter of the weight corresponds to magnetite). Also, a slight reduction in the pore volume (from 0.17 to $0.16 \mathrm{~cm}^{3} / \mathrm{g}$ ) was also observed for the composite material, compared to the parent zeolite (Fig 3.30). The fact that both the surface area and the pore volume of the zeolite are roughly maintained indicates that most of the pore mouths of the zeolitic channels remain open after inclusion of magnetite. This result makes the composite material a promising candidate as a drug-delivery vector principally for those drugs that can fit within the microporous structure. 


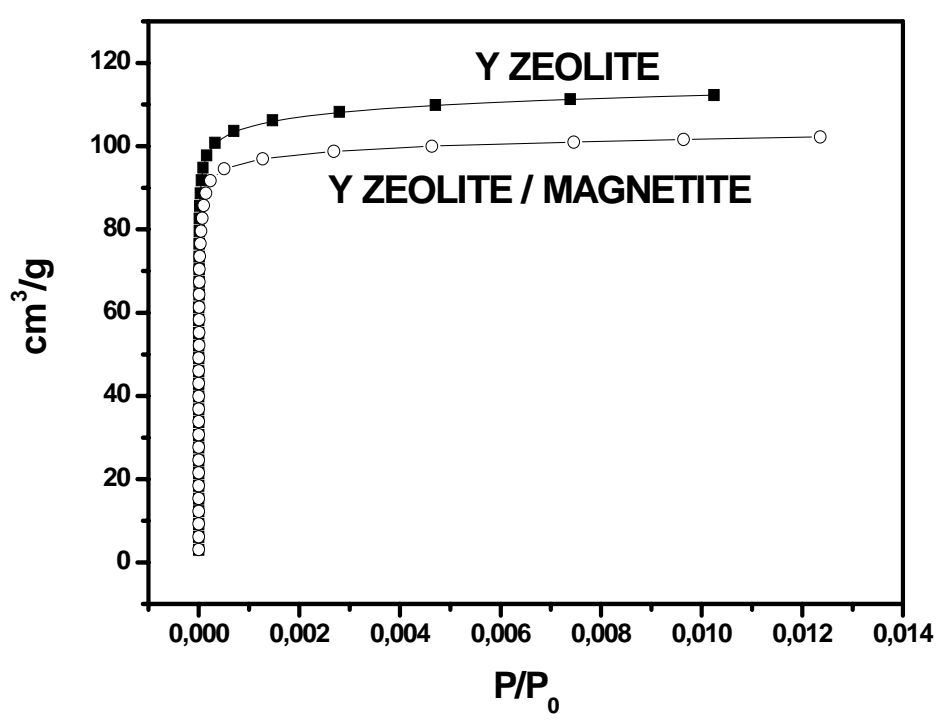

Fig. 3.29 $\mathrm{N}_{2}$ adsorption isotherm at $77 \mathrm{~K}$. pore-size distribution for the parent zeolite and for the composite material obtained after milling.

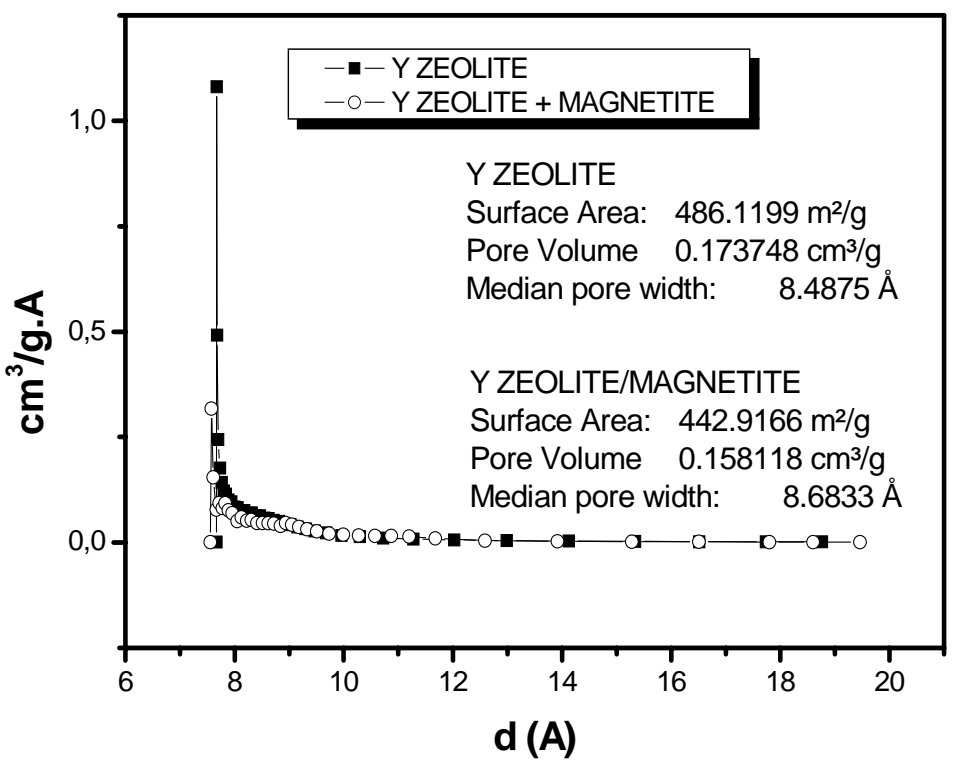

Fig. 3.30 Pore-size distribution for the parent zeolite and for the composite material obtained after milling. 


\subsubsection{IR Spectroscopy}

A complete Infra-red spectroscopy analysis has been performed for the obtained materials. It is of special interest to prove the actual chemical bond between the iron oxide particles and the silica matrix in which they are embedded. Figure 3.31 shows the IR spectra of the samples in the structural region of zeolites. The spectrum of Na-Y zeolite shows the most intense peaks in the range 1300-900 $\mathrm{cm}^{-1}$, assigned to the asymmetric T-O-T stretching vibration, with the main internal component at $1017 \mathrm{~cm}^{-1}$, together with a much less intense band with a maximum at about $790 \mathrm{~cm}^{-1}$, assigned to the symmetric stretching vibration [Sobalik1998]. Peaks at 460 and $575 \mathrm{~cm}^{-1}$ are assigned to the bending of $\mathrm{TO}_{4}$ tetrahedra and to the structurally sensitive double five-membered ring vibration, respectively [Dumitriu2003]. The $719 \mathrm{~cm}^{-1}$ band, on the other hand, is associated with the surface aluminium species [Mohamed2002].
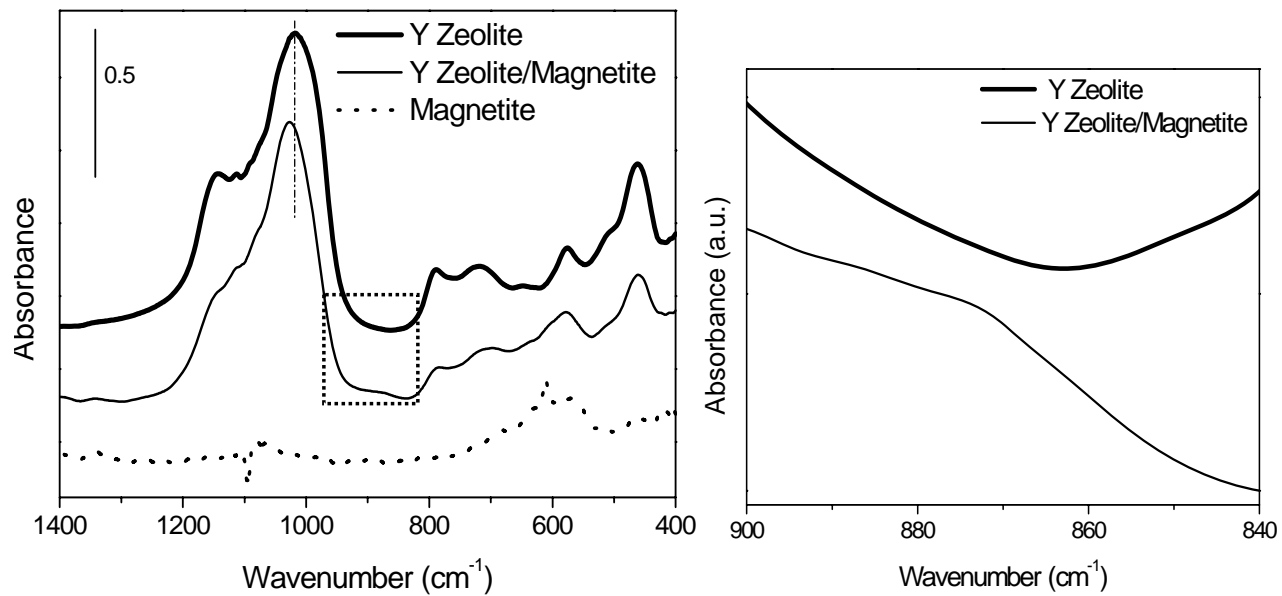

Fig. 3.31 a) FTIR spectra at room temperature for the parent materials (zeolite and magnetite) and for the composite materials obtained after milling. b) Detail of the FTIR spectra at room temperature. 
The spectrum of Y zeolite-magnetite showed spectral changes compared to that of $\mathrm{Y}$ zeolite. The band for the internal asymmetrical $\mathrm{TO}_{4}$ tetrahedra showed a shift to a higher wavenumber $\left(1027 \mathrm{~cm}^{-1}\right)$; also, a weak band at $900 \mathrm{~cm}^{-1}$ and there is a broadening of the $575 \mathrm{~cm}^{-1}$ band. Even though the increase of frequency with decreasing Al content is well known [Halasz2005], the band at $719 \mathrm{~cm}^{-1}$ remains unchanged, indicating that dealumination is not the cause of the shift. Besides, Duke et al. [Duke1995] observed $\mathrm{Al}^{3+}$ replacement in iron framework-substituted LTL zeolites, but in that case, the stretching vibration of the Si-O-T moved to lower wavenumbers with iron incorporation. On the other hand, it has been reported that the asymmetric stretching of the tetrahedron $\mathrm{TO}_{4}$ is shifted to higher wavenumbers as sodium exchange takes place [Falabella1998]. This interpretation would be consistent with our results, thus suggesting that a partial ionic exchange had taken place in the $\mathrm{Y}$ zeolite/magnetite composite.

Further evidence for Fe-Si interaction is suggested by the observation of the band at $900 \mathrm{~cm}^{-1}$. Bands around $950 \mathrm{~cm}^{-1}$ are generally attributed to the stretching vibrations of $\mathrm{SiO}_{4}$ tetrahedra perturbed by the presence of a metallic ion [Astorino1995]. Witcherlová and co-workers [Sobalik1998] explained the band at $918 \mathrm{~cm}^{-1}$ as a result of bonding of bare ions to the oxygen in the framework, causing deformation of the adjacent T-O-T bonds. This is also in agreement with results reported by Kaliaguine and co-workers [Dumitriu2003], who assigned the shoulder around $960 \mathrm{~cm}^{-1}$ in the spectra of $\mathrm{BiO}_{\mathrm{x}}$ clusters occluded in ZSM-5 zeolite to an ensemble $-(\mathrm{SiO})_{3} \mathrm{Si}-\mathrm{O}-\mathrm{Bi}$. The broadening of the double five-membered ring absorption for the composite material compared to the parent $\mathrm{Y}$ sample could also be attributed to the lattice distortion caused by iron oxide clusters in the pores [Dimitriu2003].

The FTIR spectrum of magnetite is also shown in Figure 3.32 for comparison. Undoubtedly, the observation of the $800-400 \mathrm{~cm}^{-1}$ region is not an easy task 
because of the superimposition of framework bands. The broad band around 600 $\mathrm{cm}^{-1}$ observed in the iron spectrum has been attributed to the Fe-O stretching vibrations [Giri2005]. On the other hand, Huang and Chen [Huang2005] assigned a band at $561 \mathrm{~cm}^{-1}$ to Si-O-Fe vibration.. The broadening of the $575 \mathrm{~cm}^{-1}$ band could be related to the presence of such vibrations HRTEM images showed that the magnetite nanoparticles (with diameters of 20-30 nm) generally maintain their individuality (i.e., are not agglomerated) within the zeolite/magnetite composite, and that a thin aluminosilicate coating ( $\sim 4 \mathrm{~nm}$ thick) envelops the magnetite nanoparticles, which appear associated to the larger zeolite particles $\left(200 \mathrm{~nm}^{-1} .2\right.$ $\mu \mathrm{m}$ long) (Figure 3.32). This is in agreement with the work of Zhou et al. [Zhou2002] who showed that the aggregation of the magnetic nanoparticles of $\mathrm{NiFe}_{2} \mathrm{O}_{4}$ was minimized due to the existence of the amorphous silica matrix. The appearance of the TEM micrographs suggests that a homogeneous distribution of zeolite-coated magnetite nanoparticles has been achieved, due to repeated impact and compression processes during grinding.

\subsubsection{XPS studies}

The XPS spectra of the Y zeolite-magnetite sample evidenced oxygen, silicon, aluminum, iron, sodium and carbon peaks. Atomic concentrations were calculated from the integrated intensities of the chosen peaks using ionisation cross sections provided by the instrument software. The low concentration of Fe atoms on the surface of the solid compared to the bulk material (Table 3.1) again suggests that most of the magnetite particles are coated with a zeolite layer, in agreement with HRTEM observations. The binding energy of the Fe 2p3/2 $(710.3 \mathrm{eV})$ is assigned to the stoichiometric $\mathrm{Fe}_{3} \mathrm{O}_{4}$ [Banerjee2006]. In addition, no signal of metallic iron was observed in the XPS spectrum. Binding energy levels for iron are the same in the parent material and in the zeolite/magnetite composite, indicating that there were no significant differences in the oxidation state of iron in both materials. 
Table 3.1: XPS binding energies and relative concentrations of $\mathrm{Si}$ and $\mathrm{Fe}$ on the surfaced and at the overall composite.

\begin{tabular}{|c|c|c|c|c|}
\hline \multirow[t]{2}{*}{ Solid } & \multicolumn{2}{|c|}{ Binding energies $(\mathrm{eV})$} & \multirow{2}{*}{$\begin{array}{l}\mathrm{Si} / \mathrm{Fe} \\
(\mathrm{bulk})^{1}\end{array}$} & \multirow{2}{*}{$\begin{array}{l}\mathrm{Si} / \mathrm{Fe} \\
\text { (surface) }^{2}\end{array}$} \\
\hline & Si $2 \mathbf{p}_{3 / 2}$ & Fe $2 p_{3 / 2}$ & & \\
\hline ZY-magnetite & 102.9 & 710.3 & 0.78 & 21.7 \\
\hline Magnetite & - & 710.4 & - & - \\
\hline
\end{tabular}




\section{Appendix: Magnetic saturation and size for different nanomaterials}

\begin{tabular}{|c|c|c|c|c|}
\hline Core & Shell or matrix & $\begin{array}{l}\text { Size } \\
(\mathrm{nm})\end{array}$ & $\begin{array}{l}\text { Msat } \\
\text { (emu/g) }\end{array}$ & Ref. \\
\hline Iron & Silica & $(56)$ & 163 & This work \\
\hline $\mathrm{Mn}_{1-\mathrm{x}} \mathrm{Zn}_{\mathrm{x}} \mathrm{Fe}_{2} \mathrm{O}_{4}$ & Polyaniline & 100 & 36.3 & [Lee2007] \\
\hline Nickel & Carbon & 30 & 22.5 & [He2008] \\
\hline $\mathrm{LiNi}_{0.5} \mathrm{Fe}_{2} \mathrm{O}_{4}$ & Polyaniline & 60 & 9.7 & [Jiang2007] \\
\hline Iron & Iron oxide & 12 & 85 & [Bomatí2005a] \\
\hline Iron & Silica & 300 & 125 & [Cheng2006a] \\
\hline $\mathrm{CoFe}_{2} \mathrm{O}_{4}$ & Silica & 55 & 57 & [Shang2006] \\
\hline PBMA & Fe-PGMA & 13 & 1.19 & [Bao2006] \\
\hline Magnetite & Silica & 80 & 1.2 & [Wu2004] \\
\hline Iron & Silica & 30 & 3.5 & [Bomatí2005b] \\
\hline Iron & Silica & {$[\mathrm{a}]$} & 3.7 & [Grigorieva2005] \\
\hline $\mathrm{CoFe}_{2} \mathrm{O}_{4}$ & Silica & $(50)$ & 25.3 & [García Cerdá2005] \\
\hline$\alpha-\mathrm{Fe} / \mathrm{Fe}_{3} \mathrm{C}$ & Mesoporous carbon & 50 & 30 & [Lee2005] \\
\hline $\mathrm{Ni}$ & Silica & $(75)$ & 55 & [Fu2005] \\
\hline Magnetite & Silica & 9.9 & $76[\mathrm{~b}]$ & [Gao2005] \\
\hline Magnetite & Silica & 40 & 7.5 & [Yong-Hui2005] \\
\hline $\mathrm{NiZn} / \mathrm{Fe}_{2} \mathrm{O}_{4}$ & Silica & $(16)$ & 26 & [Wu2004] \\
\hline Co & Silica & $(50)$ & 146 & [Tang2005] \\
\hline $\begin{array}{l}\mathrm{Fe}_{1-\mathrm{x}} \mathrm{RE}_{\mathrm{x}} \\
(\mathrm{RE}=\mathrm{Nd}, \mathrm{Sm}, \mathrm{Eu}, \mathrm{Tb})\end{array}$ & $\begin{array}{l}\text { Alumina and } \\
\text { maghemite }\end{array}$ & $(60)$ & 144 & [Varanda2004] \\
\hline Co & Silica & $10-15$ & 141 & [Vadala2005] \\
\hline Magnetite & Silica & 16 & 23 & [Dormer2005] \\
\hline
\end{tabular}




\begin{tabular}{|c|c|c|c|c|}
\hline Core & Shell or matrix & $\begin{array}{l}\text { Size } \\
(\mathbf{n m})\end{array}$ & $\begin{array}{l}\text { Msat } \\
(\mathrm{emu} / \mathrm{g})\end{array}$ & Ref. \\
\hline $\begin{array}{l}\text { Strontium } \\
\text { hexaferrite }\end{array}$ & Silica & - & 2.3 & [Eliseev2005] \\
\hline $\mathrm{Co}$ & Silica & $10-150$ & 130 & [Lu2005] \\
\hline $\mathrm{NiFe}_{2} \mathrm{O}_{4}$ & Silica & $(24.5)$ & 28 & [Huang2005] \\
\hline Magnetite & Silica & $360-700$ & 0.4 & [Im2005] \\
\hline Magnetite & Silica & (7.6) & 14 & [Sun2005] \\
\hline $\mathrm{Mn}_{0.65} \mathrm{Zn}_{0.35} \mathrm{Fe}_{2} \mathrm{O}_{4}$ & Silica & (18) & $38[\mathrm{~b}]$ & [Yan2004] \\
\hline Magnetite & Silica & $\begin{array}{l}(100- \\
150)\end{array}$ & 82 & [Bruce2004] \\
\hline $\mathrm{NiZn} / \mathrm{Fe}_{2} \mathrm{O}_{4}$ & Silica & $(2-25)$ & 11 & [Huang2004] \\
\hline Iron & Silica & {$[\mathrm{a}]$} & 3.7 & [Eliseev2004] \\
\hline $\mathrm{NiZn} / \mathrm{Fe}_{2} \mathrm{O}_{4}$ & Silica & $(14-22)$ & 26 & [Wu2004] \\
\hline Magnetite & Silica & 20 & 61 & [Cao2004] \\
\hline Magnetite & Silica & $50-80$ & 19.8 & [Liu2004a] \\
\hline Maghemite & SBA-15 & - & 0.3 & [Jung2004] \\
\hline $\mathrm{Co} / \mathrm{Fe}_{2} \mathrm{O}_{4}$ & Silica & 92 & 19.3 & [Silva2004] \\
\hline $\mathrm{Co} / \mathrm{Fe}_{2} \mathrm{O}_{4}$ & Silica & $(3-10)$ & 21.8 & [García Cerda2004] \\
\hline Magnetite & Silica & $100-200$ & 18.01 & [Liu2004b] \\
\hline $\mathrm{Mn}_{0.65} \mathrm{Zn}_{0.35} \mathrm{Fe}_{2} \mathrm{O}_{4}$ & Silica & $(3-21)$ & 17.15 & [Jan2004] \\
\hline Co & Silica & $(9.9)$ & 15.7 & [Jabosky2003] \\
\hline Maghemite & Silica & $(10-12)$ & 62.6 & [Xue2002] \\
\hline $\mathrm{ZnFe}_{2} \mathrm{O}_{4}$ & Silica & $(5-20)$ & 42 & [Zhou2002] \\
\hline $\mathrm{NiFe}_{2} \mathrm{O}_{4}$ & Silica & $(6-10)$ & 8 & [Mohallem2003] \\
\hline Magnetite & Mullite & (13) & 3.3 & [Morales2000] \\
\hline $\mathrm{Co} / \mathrm{Fe}_{2} \mathrm{O}_{4}$ & Silica & $(40-200)$ & 72 & [Mekala2000] \\
\hline
\end{tabular}

a] Nanowires

[b] Signal of the core

In parenthesis: size of the core only. Extracted and adapted from Fernández-Pacheco, R., Arruebo, M.; Marquina, C. I.; Ibarra, M. R., Arbiol, J.; Santamaría, J. Nanotechnology 17 (2006) 1188. 


\section{Chapter 4}

\section{Functionalisation}

This chapter comprises the results and discussion concerning the functionalisation of the magnetic particles. Silica and especially carbon are quite inert materials with hydroxyl groups on their surface. The introduction of chemical groups on these particles makes them more reactive in order to bind certain molecules. This process is known as functionalisation, and it allows particles to be tagged with a molecule that performs a specific reaction in the organism. This is, for instance, the case of an antibody that selectively recognises a tumour cell, which is especially interesting for the development of nanoparticles for biomedical applications, such as vectors in new drug design, MRI contrast agents or molecular recognition for their application in biosensors.

Also, the coating with serum proteins and the hydrophilic polymer poly(ethylene glycol) (PEG) is specially relevant for in-vivo applications. The coating of Fe@C nanoparticles has been studied with the objective of avoiding opsonisation and therefore the capture by the immune system by steric and electrostatic effects. This allows a longer circulating time in the blood stream, and a more efficient delivery of the particles to the desired target.

\subsection{Antibody binding}

In the case of drug delivery, MRI or hyperthermia applications, particles have to be tagged to recognise a selected zone of the organism. To achieve a selective 
delivery of the targeted carriers to specific organs and tissues different strategies can be used [Arruebo2007]: the most direct one is to use a specific administration route (oral, parental, interstitial, nasal, etc.) according to the organ which is targeted. Another possibility is size-dependent delivery, by means of the intrinsic porosity of the capillary vessels in the body [Okuhata1999, Weinmann2003]. Moreover, this strategy takes advantage of the fact that vascularisation changes for a tumour cell: enhanced permeability in the capillary, higher tortuosity, and less supporting tissue, and at a cellular level: expression of different surface markers [Carmeliet2000, Vicent2006]. Based on this mechanism it has been demonstrated that commercial SPION Combidex ${ }^{\circledR}$ (Advanced Magnetics Inc.) accumulates in non-cancerous lymph node tissue [Shelley2006]. Finally, the use of functional agents, like monoclonal antibodies, integrins, peptides, asialoglycoproteins, folates or polysaccharides, conjugated with the nanoparticles, produces a specific interaction with the cells of the targeted organ or tissue [Morawski2005]. In 1996 Suzuki et al. [Suzuki1996] described the development of targeted-directed magnetic resonance contrast agents using monoclonal antibody-conjugated magnetic particles. Since then, several scientific papers concerning targeted ironoxide contrasts agents for MRI have been published [Artemov2003, Ahrens2003, Pirko2004, Funovics2004]. Huh et al. [Huh2005] have reported magnetic nanocrystals conjugated to a cancer-targeting antibody (herceptin), and subsequent utilisation of these conjugates as MRI probes. They have successfully demonstrated the monitoring of in-vivo selective targeting events of human cancer cells implanted in live mice. Moore et al. [Moore2004] reported the synthesis and characterization of targeted contrast agents, which show the specific recognition in vivo of uMUC-1 antigen in an animal model of human cancer. At this time, targeted contrast agents for MRI are already commercialized (i.e., MagNaGel ${ }^{\circledR}$ Alnis Biosciences Inc.) 
In order to develop a contrast agent with potential active targeting properties, carbon and silica-coated magnetic nanoparticles have been functionalised with an antibody. The antibody anti-hCG was chosen because it is widely studied due to its common use in pregnancy testing kits. Ongoing work is aimed at the use of antibodies that are able to recognize tumour-associated antigens as well as growth factors (anti-VEGF, anti-VCAM).

\subsubsection{Functionalisation with carboxyl and amine groups}

The strong coupling between carboxyl and amine groups via a carbodiimide bond is responsible for many interactions between biomolecules. It is the case of the peptidic bond between amino-acids to form a protein. In order to make use of this coupling, particles can be designed to carry either amino or carboxyl groups on the surface to be linked to a desired complementary molecule. The reflux in an organic solvent of the particles in the presence of APTES (aminopropyltroethoxysilane) leads to the substitution of hydroxyl groups on the inorganic surface (silanol, $\mathrm{Si}-\mathrm{OH}$ in the case of silica coating) by aminosilane groups (figure 4.1a).

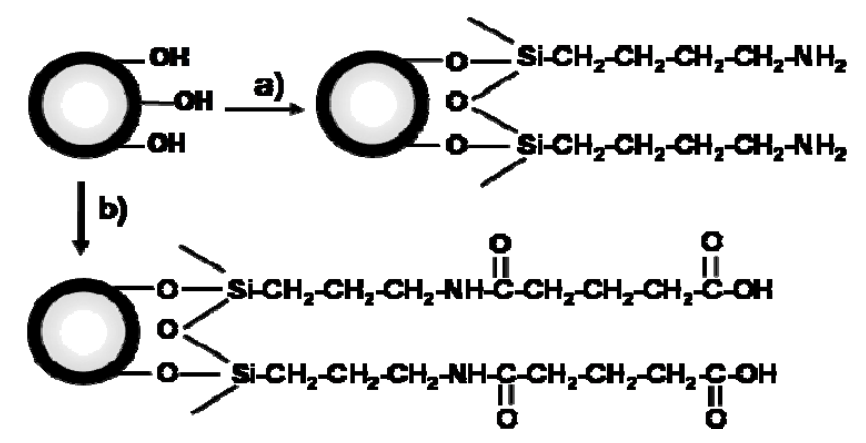

Figure 4.1 Schematic diagram of the superficial components of the biofunctionalised nanoparticles. Carbon or silica coatings initially have hydroxyl groups on their surface, that can be substituted to yield: a) Amine functionalised silica coated iron oxide nanoparticles, b) carboxyl functionalised silica coated iron oxide nanoparticles. 
These particles can then react towards carboxyl-modified surfaces or other amines, via the glutaraldehyde bond. In the presence of glutaric anhydride the amine group of the APTES reacts with the anhydride to yield a carboxyl-modified product (figure 4.1.b). Carboxyl-modified particles can then be used for their linkage to amino terminal-groups. This functionalisation reaction has proved to be rather more effective than alternative procedures, like the reflux in the presence of a nitric acid, which has been used for the introduction of carboxyl groups on the surface of carbon nanotubes. This method was tested for the carbon-coated nanoparticles, but the introduction of carboxyls was scarce.

Different solvents were tested to obtain more stable functionalised products. Many synthesis reactions of nanoparticles involve the use of organic solvents. However, any in-vivo application requires the particles to be dispersed in an aqueous solution at their final stage. Hence any traces of organic components must be eliminated. Dimethylformamide (DMF) is toxic and difficult to remove from the reaction, therefore toluene $\left(\mathrm{CH}_{3}-\mathrm{C}_{6} \mathrm{H}_{5}\right)$.was finally chosen for a complete solubilisation of the initial reagents and a better dispersion within an aqueous medium.

\subsubsection{XPS characterisation of functionalised surfaces}

To demonstrate the actual presence of amino and carboxyl groups on the particles, the surface was studied by infrared and photoelectron spectroscopy. For the amine-functionalised nanoparticles, FTIR analysis could not provide evidence of the presence of amino groups in the region of $1650-1500 \mathrm{~cm}^{-1}$ because of the interference of adsorbed water on the surface. In contrast, for the carboxylmodified nanoparticles this technique clearly showed the presence of absorption bands in the range from 1380 to $1550 \mathrm{~cm}^{-1}$ which are associated with the presence of $\mathrm{COO}^{-}$groups. 
Regarding XPS analysis, the main elements detected before functionalisation of the iron oxide-silica particles were $\mathrm{Si}$ and $\mathrm{O}$, in addition to the usual adventitious carbon. Concentrations of $\mathrm{Fe}$ and $\mathrm{N}$ were less than 0.6 atom \%. The peak positions detected for $\mathrm{Fe} 2 \mathrm{p}_{3 / 2}$ and $2 \mathrm{p}_{1 / 2}$ at 710.8 and $724.3 \mathrm{eV}$, respectively are consistent with the presence of iron in magnetite [Shah2002]. The particles were again analysed by XPS after the chemical functionalisation and before antibody coupling showing the presence of amine and carboxyl groups (see Table 4.1).

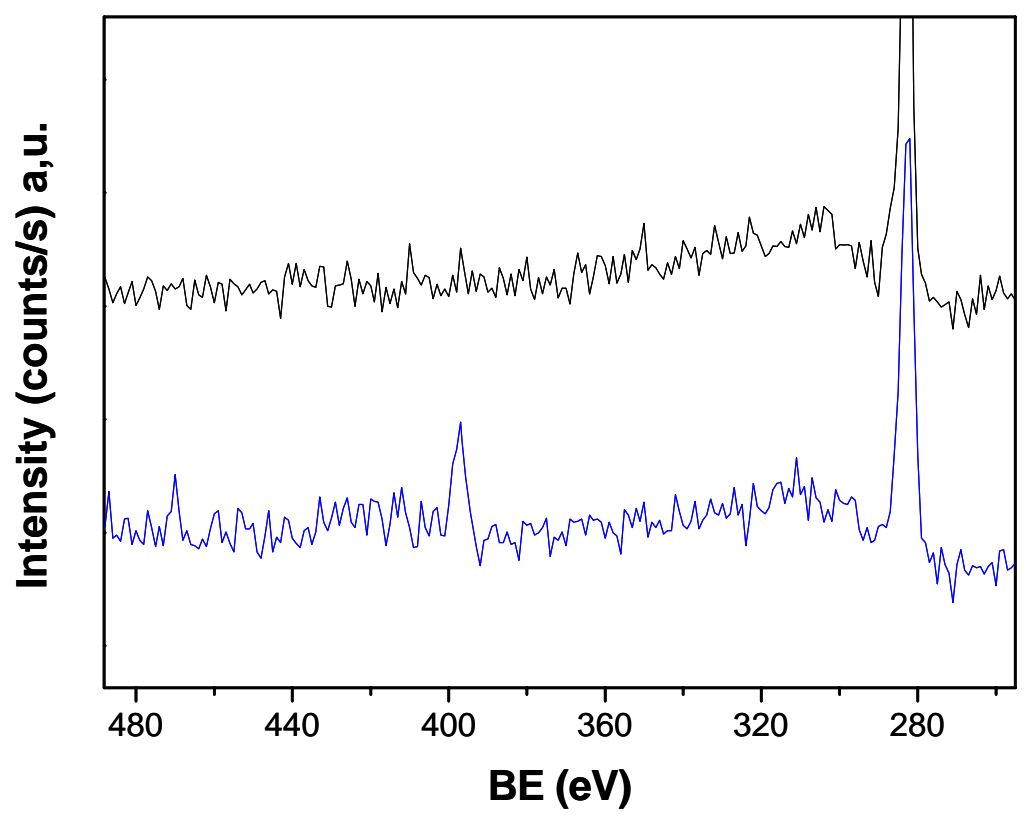

Figure 4.2: XPS spectra of the faminated $\mathrm{Fe}_{3} \mathrm{O}_{4} @ \mathrm{SiO}_{2}$ nanoparticles. The peaks at $397 \mathrm{eV}$ and $399 \mathrm{eV}$ correspond to $-\mathrm{NH}_{2}$ and $\mathrm{R}_{2}-\mathrm{NH}$ bonds [García Fierro2003]. Bare Fe/SiO2 black - NH2 functionalised Blue

The amine-modified particles showed a broad band corresponding to $\mathrm{N} 1 \mathrm{~s}$ core-level spectrum, which was deconvoluted into two contributions (Fig. 4.2, Table 4.1). The peak at $399.3 \mathrm{eV}$ would correspond to $-\mathrm{NH}_{2}$ species while the highest Binding Energy component could be attributable to decomposition 
products of the precursor, i.e. $\mathrm{R}_{2}-\mathrm{NH}$, were $\mathrm{R}$ is a hydrocarbon chain [Arroyo2003]. C 1s (Table 4.1) presents two components, associated to C-C and C$\mathrm{N}$ bonds; oxygen and Si spectra show peaks consistent with these results.

Table 4.1: Binding energies and Full width at half maximum (FWHM) of $\mathrm{N} 1 \mathrm{~s}$ and $\mathrm{C}$ $1 \mathrm{~s}$ peaks for the amine and carboxyl functionalised nanoparticles before antibody coupling

\begin{tabular}{ccccr}
\cline { 2 - 5 } & \multicolumn{2}{c}{ N 1s } & \multicolumn{2}{c}{ C 1 s } \\
\cline { 2 - 5 } & $\begin{array}{c}\text { Binding energy and } \\
(\mathrm{FWHM}) \mathrm{eV}\end{array}$ & $\begin{array}{c}\text { Peak } \\
\text { percentage }\end{array}$ & $\begin{array}{c}\text { Binding energy } \\
\text { and (FWHM) } \mathrm{eV}\end{array}$ & $\begin{array}{c}\text { Peak } \\
\text { percentage }\end{array}$ \\
\hline Amine & $399.3(1.5)-\mathrm{NH}_{2}$ & $74 \%$ & $284.9(1.4) \mathrm{C}-\mathrm{C}$ & $86 \%$ \\
functionalised & $401.3(1.7) \mathrm{R}_{2}-\mathrm{NH}$ & $26 \%$ & $286.2(1.2) \mathrm{C}-\mathrm{N}$ & $14 \%$ \\
nanoparticles & & & & \\
\hline Carboxyl & $399.8(1.5)-\mathrm{NH}_{2}$ & $5 \%$ & $284.8(1.4) \mathrm{C}-\mathrm{C}$ & $73 \%$ \\
functionalised & $401.6(1.8) \mathrm{R}_{2}-\mathrm{NH}$ & $95 \%$ & $286.0(1.5) \mathrm{C}-\mathrm{O}$ & $13 \%$ \\
nanoparticles & & & and $/$ or C-N & $14 \%$ \\
& & & $288.0(1.3) \mathrm{ROC}=\mathrm{O}$ & \\
\hline
\end{tabular}

The carboxyl-functionalised nanoparticles showed a broad peak, which can be associated with the core level spectra of $\mathrm{C} 1 \mathrm{~s}$ were resolved into three peaks (Table 4.1). The main one, at $284.8 \mathrm{eV}$ was attributed to carbon atoms bonded to carbon neighbours [Thomé2006], while the $286.0 \mathrm{eV}$ component can be ascribed to C-N bonds [Arroyo2003] and/or to $\mathrm{C}=\mathrm{O}$ bonds also present the carboxyl-functionalised nanoparticles. The last component $(288.0 \mathrm{eV})$ would confirm the presence of $\mathrm{ROC}=\mathrm{O}$ groups. Regarding the $\mathrm{N}$ 1s core-level spectrum, the analysis was similar to that of the amine-modified particles, with deconvolution into two contributions, one at $401.6 \mathrm{eV}$ corresponding to $\mathrm{R}_{2}-\mathrm{NH}$ bonds, and a second component attributed to amine groups not attached to the carboxylic compound [Arroyo2003]. It is important to point out that the relative abundance of nitrogen in amine groups is 
very low ( 5 atom \%). The analysis of the rest of the elements present did not provide reliable information and are not included in the table.

\subsubsection{Binding to the antibody anti-hCG}

After the grafting of functional groups, the anti-hCG antibody was linked to the surface of the particles by two different mechanisms. In the case of carboxylmodified particles, the antibody was grafted via the carbodiimide bond (Fig. 4.3). An acylisourea unstable intermediate is formed and it rapidly reacts in the presence of an amino group to yield a strong covalent bond between the particle and the antibody. In the case of the amino-modified particles, the antibody was linked via the glutaraldehyde bond (Fig. 4.4). An aldehyde activated sphere is formed on the first step that reacts with the amino group of the antibody [Bangs2002].

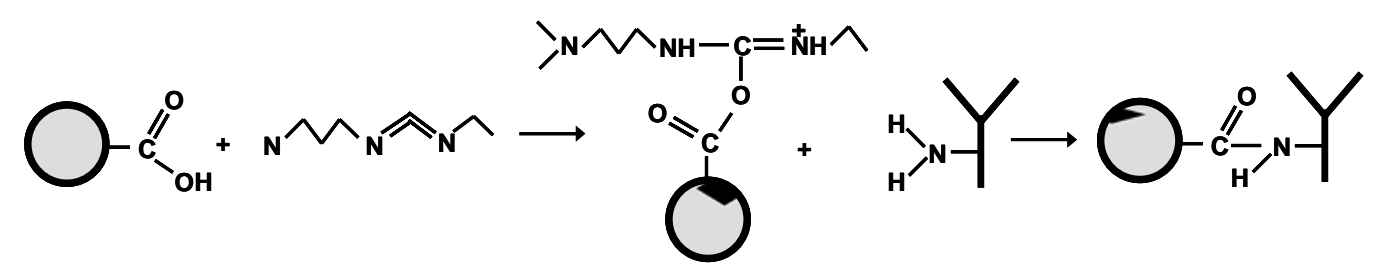

Figure 4.3: Coupling between a carboxyl-modified particle and an antibody via the carbodiimide bond. Even though the antibody is displayed with simplified Y-shape, the binding is randomly-oriented.

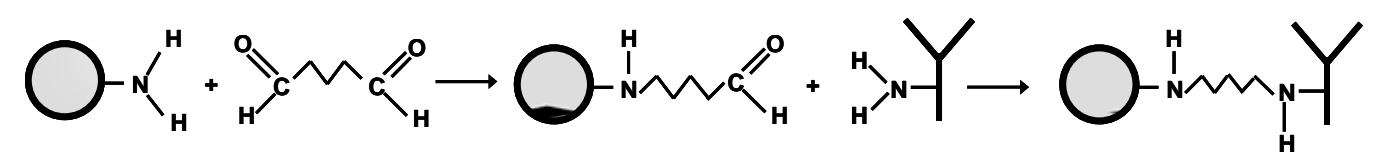

Figure 4.4: Coupling between an amino-modified particle and an antibody via the glutaraldehyde bond. The binding is randomly-oriented. 
To verify that a suitable attachment of the antibody had been achieved for either procedures, a standard immunoassay test was carried out. The human chorionic gonadotropin hormone (hCG) is detected in a nitrocellulose membrane where the complementary anti-antibody had been previously immobilised. The specific binding streptavidin-biotin was used as the control line in the membrane to assure the quality of the test. One end of the test strip was immersed in this suspension and then the elution flow proceeded by capillarity. Capture of the antibody-functionalised nanoparticles produced a dark band in the region of the test strip where the complementary anti-antibody had been immobilised (Fig. 4.5). Experimental details are fully described in section 2.3.2, Page 64.

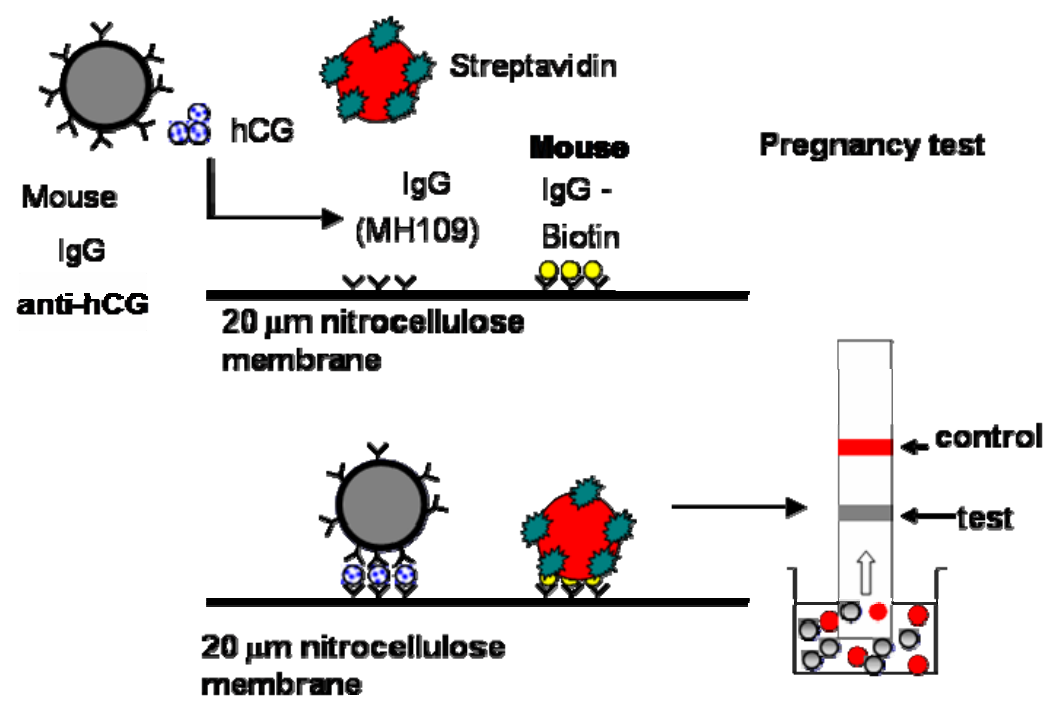

Figure 4.5 Schematic diagram showing the method of the detection of the antibody. Visual detection of the antibody-conjugated nanoparticles by means of capture in a nitrocellulose membrane containing the complementary antibody. The specific binding streptavidin-biotin is used as the control line in the membrane to assure the quality of the test.

The intensity of colour can be photographically assessed and related to the concentration of nanoparticles in the suspension and with the amount of hCG in the 
solution. The interaction between Human chorionic gonadotripin and its complementary anti-hCG antibody was chosen as a model because it has been widely studied because of its common use in pregnancy test kits.

In the immunochromatographic assays, also termed lateral flow tests, control assays using antibody-conjugated superparamagnetic nanoparticles were also performed without adding the hCG hormone to the buffer dispersion. Without the hormone in the media no band was detected (optically or magnetically), proving the specific interaction between the hormone and the anti-hCG antibodies.

The sensitivity of the method was tested by adding different amounts of the hCG hormone to the nanoparticle buffer dispersion. The dark band observed in the nitrocellulose membrane shows an intensity that increases with the concentration of nanoparticles in the suspension and with the amount of hCG in the solution. Both the magnetic and colorimetric signals of the nanoparticles attached to the corresponding specific antibody directed against hCG (previously immobilised in the nitrocellulose strip) are proportional to the amount of antibody attached to the nanoparticles. It should be noticed that with the standard biochemical procedure followed in this work to attach the antibody to the external surface of the nanoparticles a random orientation of the antibody was obtained. In a recent work we achieved a more selective orientation, and therefore a more effective interaction by coupling the Fc zone of the antibody to the hybrid superparamagnetic nanoparticles [DeTeresa2008]. 


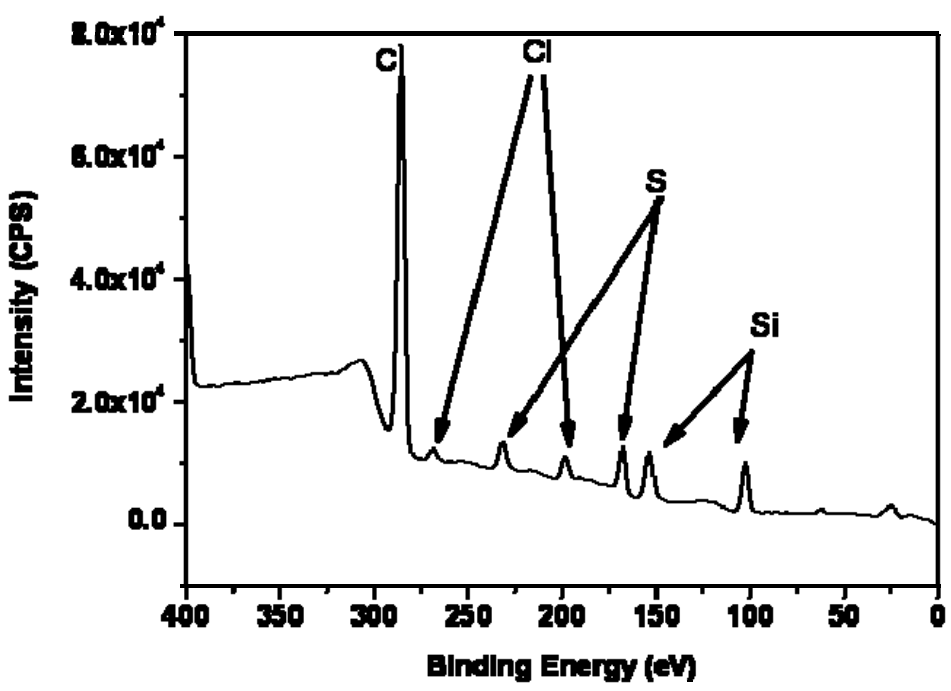

Figure 4.6. XPS spectra of the monoclonal antibody-conjugated silica-coated magnetite nanoparticles, proving the presence of sulphur and the binding of the antibody to the surface of the nanoparticles.

The presence of the anti-hCG monoclonal antibody IgG (anti-MH95) in the amine functionalised nanoparticles after the washing protocol was also demonstrated by means of XPS analysis. The main elements present in the surface were $\mathrm{C}, \mathrm{O}, \mathrm{N}, \mathrm{Na}, \mathrm{Si}, \mathrm{S}$, and $\mathrm{Cl}$ (Table 4.2). After covalent binding to the antibody, the functionalised nanoparticles were gently washed 3 times in the washing buffer, and then separated using a permanent magnet, dried and analysed. Sulphur was detected in the spectrum (Fig 4.6, Table 4.2). This sulphur can only come from the antibody because the anti-hCG monoclonal antibody anti-hCG contains inter- and intra-chain disulfides (Fig. 4.7). Detected sodium would also come from the antibody since it was not present previously in the amine-functionalised particles. Similar results of XPS analysis were obtained for the carboxyl-functionalised nanoparticles, confirming the presence of antibody attached to their surfaces. 


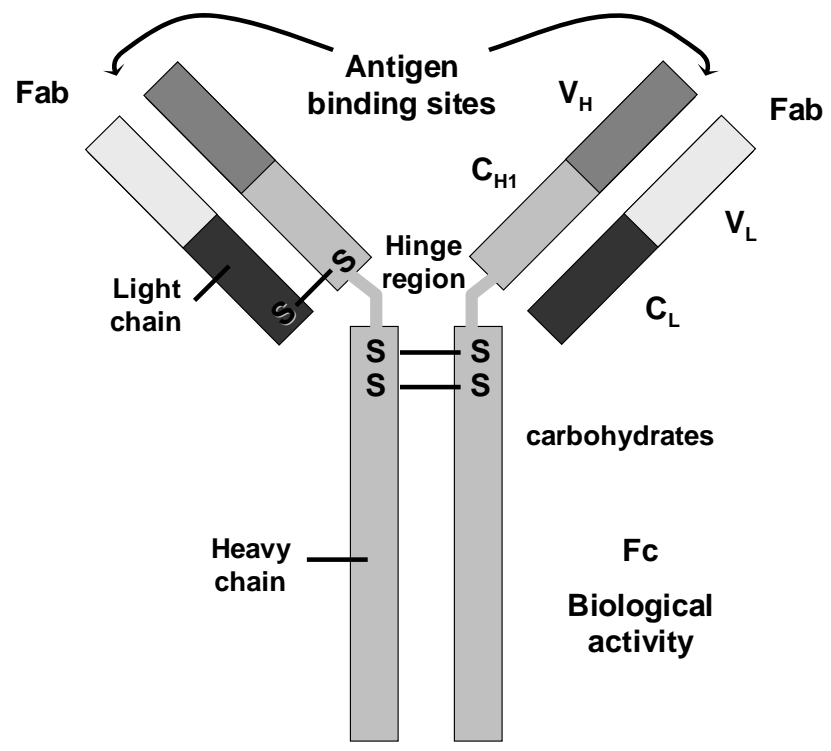

Figure 4.7: Scheme of an antibody. The presence of intra- and inter- disulphide bridges is detected by XPS analysis.

Table 4.2: XPS analysis of the amine-functionalised nanoparticles after antibody attachment

\begin{tabular}{ccc}
\hline Element & $\begin{array}{c}\text { Binding Energy. } \\
\text { (FWHM) eV }\end{array}$ & $\begin{array}{c}\text { Atomic } \\
\text { Concentration (\%) }\end{array}$ \\
\hline C 1s & $284.9(2.6)$ & 55 \\
O 1s & $531.4(2.0)$ & 25 \\
N 1s & $398.7(1.8)$ & 9 \\
Si 2p & $102.3(1.8)$ & 7 \\
S 2p & $\mathbf{1 6 7 . 2 ( 2 . 3 )}$ & $\mathbf{3}$ \\
Na 1s & $1070.6(1.4)$ & 1 \\
Cl 2p & $196.7(2.6)$ & 1 \\
\hline
\end{tabular}




\section{2 Coating with serum albumin and PEG}

Phagocytosis is a cellular phenomenon that describes the process in which phagocytes, which are specialised cells such as macrophages, destroy viruses and foreign particles in blood. Phagocytes then play a very important role in the immune system. Unfortunately, phagocytes are also a major limitation for the intravenous delivery of nanoparticles in any in-vivo application. Nanoparticles become recognizable to the cells of the mononuclear phagocyte system, and are subsequently cleared from circulation by phagocytosis, through a process called opsonisation. During this process particles circulating in blood are coated with molecules which make them more "appealing" to bind to receptors on phagocytes. Antibodies, especially immunoglobulins and complement proteins like C3b can opsonise and are therefore are referred to as opsonins. The IgG antibodies bind to the antigens by the Fab region leaving the Fc region sticking out. Phagocytes have Fc gamma receptors and therefore they can bind to the coated molecules and internalize them. The complement fragment, $\mathrm{C} 3 \mathrm{~b}$, binds nonspecifically to foreign organisms. Phagocytes also have receptors for $\mathrm{C} 3 \mathrm{~b}$ on their surface. The antibodies and $\mathrm{C} 3 \mathrm{~b}$ tag the microorganisms or nanoparticles for destruction by phagocytes.

If a particle is negatively charged, there is an electrostatic repulsion between the membrane of a phagocytising cell, also charged with negative groups, and the particle. That would avoid the mechanism of phagocytosis by the rethiculoendothelial system. However, during the process of opsonisation, particles are bound by opsonins, enhancing macrophage capture, since phagocytic cells express receptors that bind opsonin molecules. Moreover, opsonisation of a particle and subsequent binding to an activated phagocyte will cause increased expression of complement receptors on neighbouring phagocytes, accelerating the whole process. In fact, without opsonisation of the antigen phagocytic binding would practically disappear. 
In order to improve the in-vivo survival rate of nanoparticles, the goal is to design a long circulating carrier with a functionalised surface in order to avoid opsonin adsorption and the subsequent clearance from the body by phagocytic cells. The physicochemical characteristics of a nanoparticle such as surface charge, size, functional groups, and hydrophilicity can affect its uptake by the cells of the phagocyte system. A neutral (or negatively charged) and hydrophilic surface can lead to a longer half-life in the blood circulation.

Two strategies have been developed within this work to achieve this goal: the adsorption of serum proteins on the magnetic nanoparticles, and the coating with a hydrophilic polymer like poly(ethylene glycol) (PEG). The conjugation of Bovine Serum Albumin (BSA) to the surface of the particles has the objective of avoiding opsonisation by steric and electrostatic effects. The binding of serum proteins yields the particles electronegative at the physiological $\mathrm{pH}$, impeding the specific recognition of receptors on the macrophages' membrane.The incorporation of PEG on the surface of nanoparticles formulation has yielded a decreased uptake by cells of the RES and an increased circulation time to effectively target diseased cells. PEG molecules on the surface of a nanoparticle reduce the adsorption of opsonins and other serum proteins by a steric repulsion effect. PEG molecules on the surface of a nanoparticle will form a dynamic molecular cloud over the particle surface. This cloud of mobile and flexible PEG molecules produces a repulsive effect, which makes energetically unfavourable for proteins to be adsorbed onto PEG molecules. Pishko and coworkers determined a relationship between uptake by macrophages and physicochemical properties like surface charge and the hydrophilicity of core-shell nanoparticles, proving that a low negative to neutral and hydrophilic nanoshell can reduce uptake of coreshell nanoparticles in-vitro. They used a layer-by-layer (LbL) assembly process to produce core-shell polymeric nanoparticles where fluorescent nanobeads (to simulate a solid drug core) were encapsulated in macromolecular nanoshells. The nanoshell composed of 
multilayered polyelectrolytes was modified with PEG of varying molecular weights $(2000,5000$, or $20,000 \mathrm{Da})$ to form a hydrophilic and long-term circulating nanoparticle. [Zahr2006]. The choice of the utilised PEG is also vital. Zahr and coworkers [Zahr2006] attached chemically PEG chains of varying molecular weights $(2000,5000$ and $20000 \mathrm{Da})$ to polymeric particles to yield and electrically neutral and hydrophilic surface of core-shell nanoparticles. They studied the different invitro uptake by macrophages and protein adsorption depending on the utilised PEG.

The molecular weight of PEG and thus its chain length has an influence on phagocytosis. The hydration of coiled, flexible PEG chains from 1500 to $5000 \mathrm{Da}$ can help to avoid the approach of proteins [Stolnik1995]. Napper and co-workers suggested that the stability of the polymer coverage could determine the distribution of a colloidal carrier in a biological environment [Hunter1983].

The covalent attachment of a neutral polymer, such as PEG, should produce a neutralisation in charge at the surface of the particle. The measurement of zeta potential before and after the introduction of PEG can then be a useful tool to determine the effectiveness of the attachment of the polymer to the particle. Besides, metyhlated PEG (mPEG) has been previously used to decrease immunogenicity and proteolysis of a number of various macromolecules, like albumin, interferon- $\gamma$, interleukin-1, colony stimulating factor, and superoxide dismutase, as well as to increase their circulation half-lives [Monfardini1998].

There are three common ways to attach PEG molecules to the surface of a particulate carrier: incorporation of PEG during the synthesis of the particles; covalent attachment of activated PEG molecules to the reactive surface of previously produced particles; and physical adsorption of appropriate PEG derivatives, such as poly-(lactyde-co-glycolide)-PEG to nanospheres. Particles with 
poorly reactive surfaces and the rapid desorption of physically adsorbed PEG derivatives are severe limitations to these processes. Desorption of PEG leads to the formation of steric barriers when particles are in contact with blood, a process leading to quick capture of the particles by macrophages [Moghimi2001].

\subsubsection{Bovine Serum Albumin Coating}

BSA was incubated with the particles at different concentrations to estimate the maximum capacity of adsorption. Sodium Phosphate buffer was used as the dispersion medium with a low ionic force (concentration of $\mathrm{NaCl}=0.01 \mathrm{M}$ ) and a $\mathrm{pH}=8$ to simulate mild conditions. Particles were first dispersed in the buffer and sonicated with an ultrasound probe to remove aggregates and achieve an optimum redispersion. BSA was added and the whole system was incubated with sonication for 1h. Particles conjugated with BSA were separated by centrifugation, and the remaining protein was detected by Vis-uv spectroscopy using the reagent BCA for protein quantification. The BCA Protein Assay is based on bicinchoninic acid (BCA) for the colorimetric detection and quantitation of total amounts of protein. The method combines the reduction of $\mathrm{Cu}^{2+}$ to $\mathrm{Cu}^{+}$by proteins in an alkaline medium with the highly sensitive colorimetric detection of the cuprous cation. The product of the reaction is a purple-coloured solution formed by the chelation of two molecules of BCA with one cuprous ion. This water-soluble complex exhibits a strong absorbance at $562 \mathrm{~nm}$, nearly linear with increasing protein concentrations over a broad working range (approximately from 20-2,000 $\mu \mathrm{g} / \mathrm{ml}$ ). Remnant BSA reduces $\mathrm{Cu}^{2+}$ to $\mathrm{Cu}^{+}$, and these ions are detected by conjugation with the BCA. The absorbance of the resulting liquid is measured at $562 \mathrm{~nm}$, and the concentration of serum albumin bound to the surface of the particles can be calculated by difference.

Increasing quantities of the protein were added to a fixed concentration of nanoparticles in an aqueous suspension at a $\mathrm{pH}=8$ to study the loading capacity of 
the particles. Maximum adsorption was achieved for a concentration of $2 \mathrm{mg} / \mathrm{ml}$. After saturation increasing concentrations of added protein do not mean an increase in adsorbed BSA (Fig. 4.8).

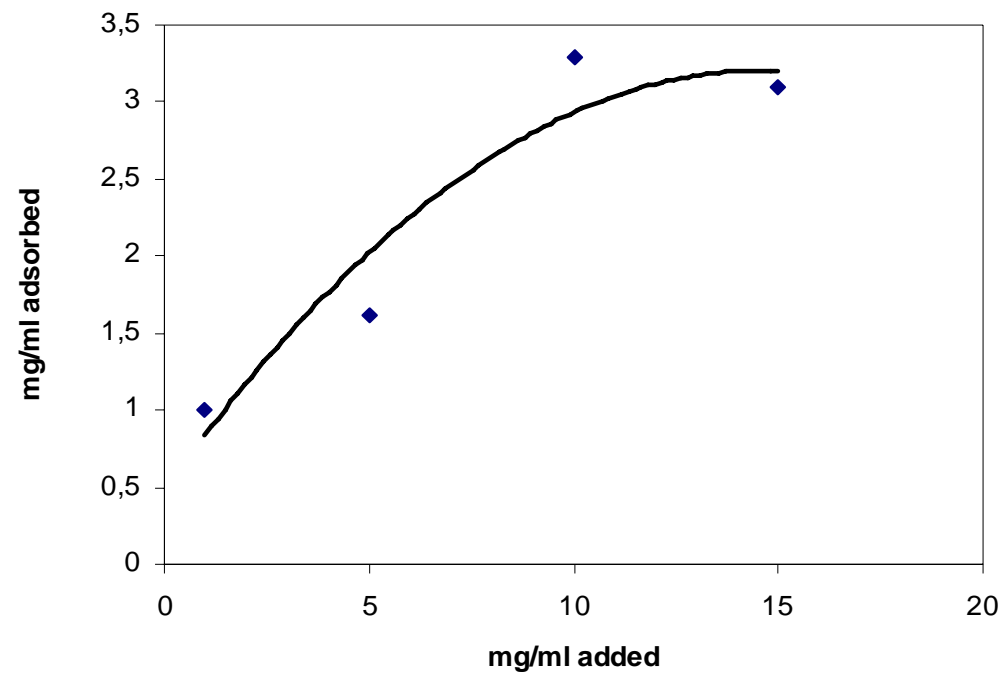

Figure 4.8 Loading capacity of the Fe@C particles with BSA. Maximum adsorption was achieved for a concentration of $2 \mathrm{mg} / \mathrm{ml}$.

In order to avoid rejection reactions by the rabbits against a foreign protein, the serum BSA was substituted by commercial Rabbit Serum Albumin (RSA) for the in-vivo administration of particles. Ideally serum albumin should be extracted from the same animal in which it is going to be injected, but this was discarded because of the intrinsic difficulties in isolating the protein for each case. The adsorption of the protein was done following the same procedure as for the bovine serum albumin, using the maximum concentration of adsorbed protein.

\subsubsection{Poly(ethyleneglycol) coating}

The other strategy was the pegylation of the particles. An amphiphilic molecule, Poly (maleic anhydride-alt-1-octadecene) (PMAO), with a long 
hydrophobic chain on one side adsorbed on the carbon hydrophobic surface, leaving its carboxyl, hydrophilic groups on the other side covalently bound to the amino groups of a methoxy-modified Polyethyleneglycol chain (PEG). Once the particles were functionalized with PEG, the presence of the polymer would prevent the adsorption of big proteins of the blood and hence delay the recognition of the particle from the immune system [Yu2006].

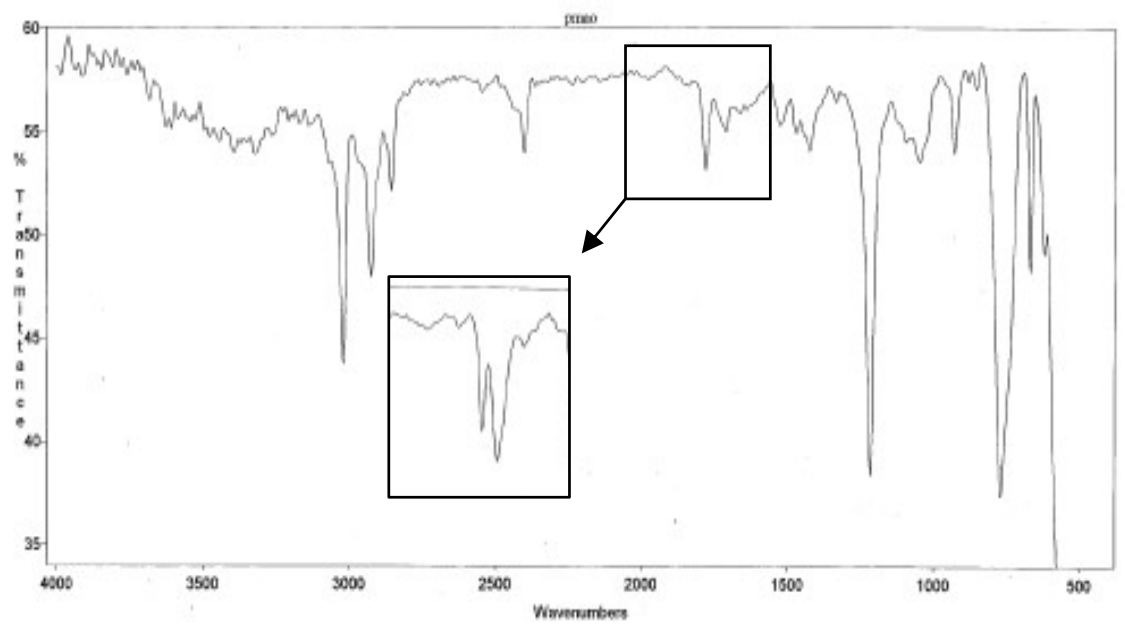

Figure 4.9: IR spectrum of the PMAO reagent. Inset: the decrease of the $1775 \mathrm{~cm}^{-1}$ peak and the increase of the $1715 \mathrm{~cm}^{-1}$ peak are characteristic of the decomposition of anhydride and the release of carboxyl groups, respectively.

The formation of the copolymer after reaction of PMAO and PEG was followed by Infra-Red spectrometry. The decrease of the $1775 \mathrm{~cm}^{-1}$ peak and the increase of the $1715 \mathrm{~cm}^{-1}$ peak are characteristic of the decomposition of anhydride and the release of carboxyl groups, respectively [Atici2000, Kang2001]. The rest of the main peaks of both initial reactants are present at the final product (Fig. 4.9). 
Table 4.3: XPS analysis of the Fe@C particles before and after pegylation

\begin{tabular}{cccc}
\cline { 2 - 3 } & \multicolumn{3}{c}{ Binding energy eV } \\
& C 1s & N 1s & O 1s \\
\hline Bare Fe@C particles & $284.9 \mathrm{eV}$ & \\
Pegylated Fe@C & a) $285 \mathrm{eV} \mathrm{C}-\mathrm{C}$ & $397 \mathrm{eV} \mathrm{NH}_{2}$ & $534 \mathrm{eV}$ \\
particles & b) $289 \mathrm{eV} \mathrm{C}-\mathrm{O}$ & & \\
\hline
\end{tabular}

XPS studies prove that the polymer effectively coats the particles. By XPS the presence of nitrogen on the surface of the particles was detected. This element can only appear due to the presence of the amino groups that result form the coating of the particles with the polymer. This can be checked comparing the spectrum of the PEG-PMAO functionalised particles with a blank spectrum of an Fe@C sample (Fig. 4.10) at the $\mathrm{N} 1 \mathrm{~s}$ region, $397 \mathrm{eV}$. Also, the $\mathrm{C} 1 \mathrm{~s}$ and $\mathrm{O} 1 \mathrm{~s}$ spectra reveal the functionalisation of the surface of the particles. The main carbon peak at $285 \mathrm{eV}$ is the hydrocarbon or the C-C peak. Once the particle has been functionalised, there is a second peak, shifted at $289 \mathrm{eV}$, the C-O peak (Fig. 4.11), characteristic of PEG coupling [Popat2004]. The apparition of a secondary peak at $534 \mathrm{eV}$ at the $\mathrm{O} 1 \mathrm{~s}$ spectrum is another indication of the effective coupling reaction of PEG to the surface of the particles (Fig. 4.12). In all the cases, a $5 \mathrm{KV}$ Ar ion etching was performed for different times to probe the chemical composition of each sample. No significant differences were observed as the outer surface was etched, except for the region of $\mathrm{C} 1 \mathrm{~s}$ in the case of the PEG-PMAO functionalised particle: the peak at $289 \mathrm{eV}$ slightly shifts, whereas a second peak at $292 \mathrm{eV}$ appears, probably corresponding to the carbonous chain of the PMAO. 

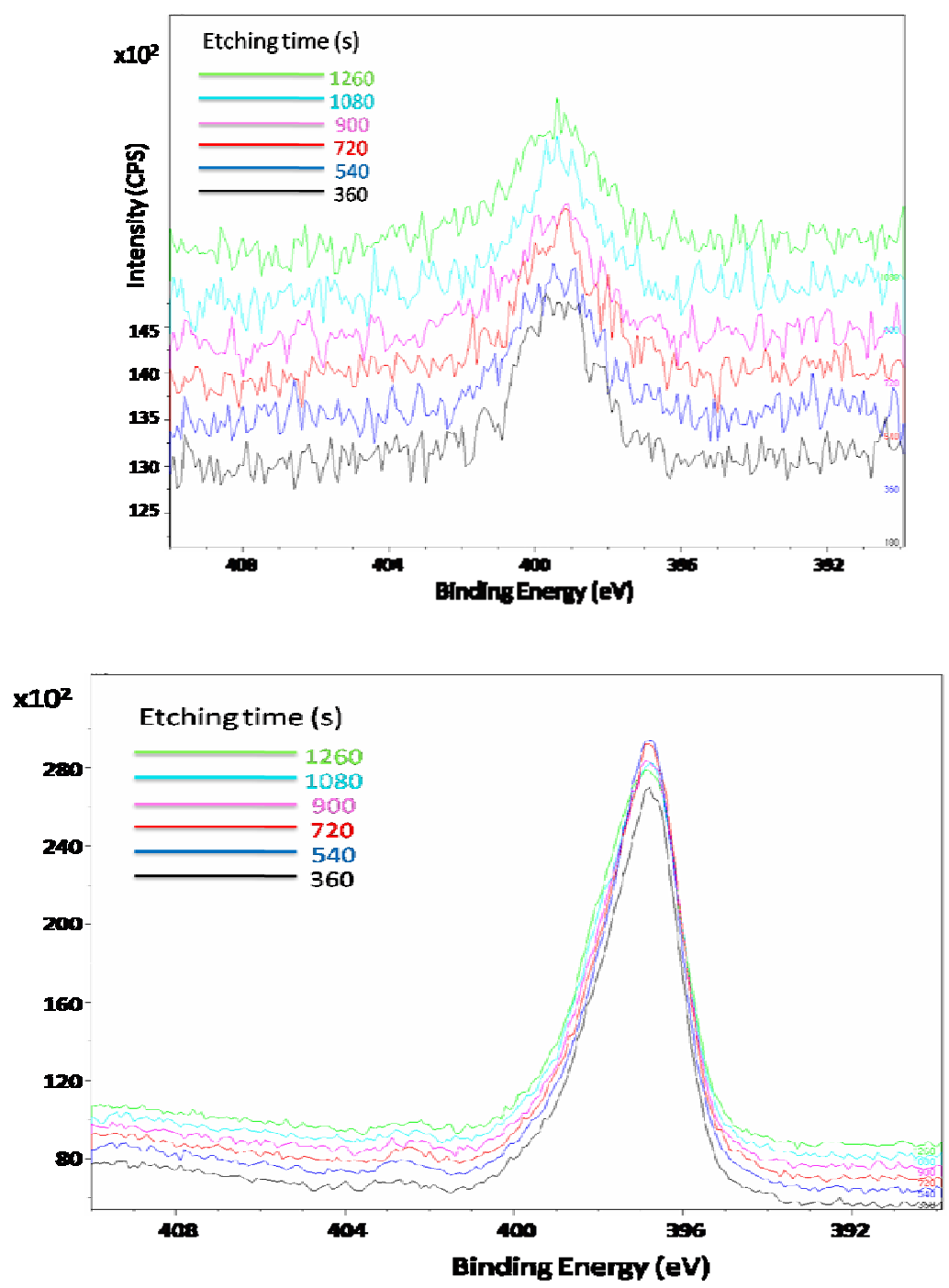

Figure 4.10: Regions of nitrogen 1s as a function of etching time a) bare Fe@C. b) PEGPMAO functionalisedFe@C. 

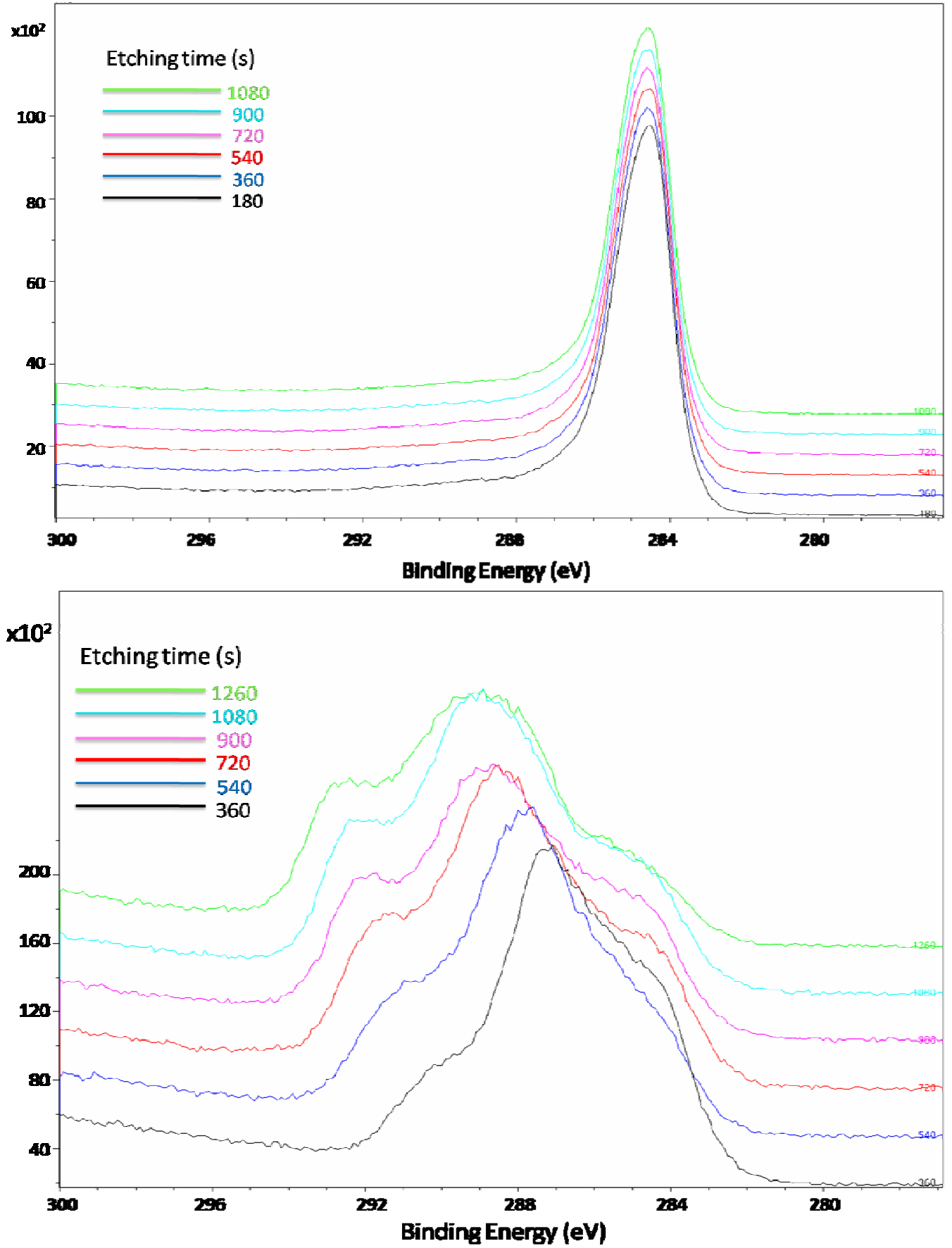

Figure 4.11: Regions of carbon 1s as a function of etching time a) bare Fe@C b) PEGPMAO functionalised Fe@C. 

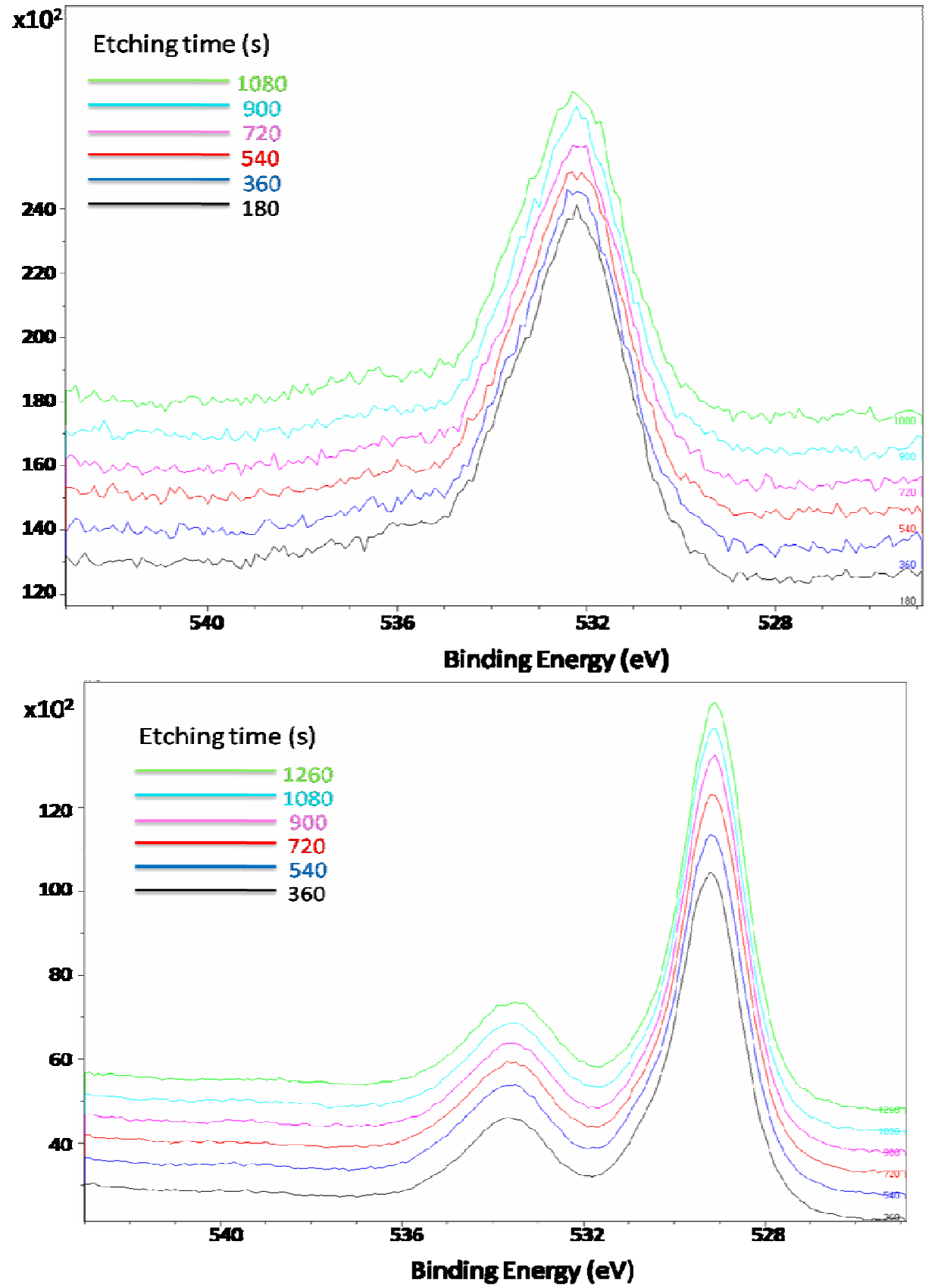

Figure 4.12: Regions of oxygen 1s as a function of etching time a) bare Fe@C b) PEGPMAO functionalised Fe@C. 
Further evidence of a proper coating of the particles has been obtained studying their ability to adsorb the protein BSA. Before the treatment naked carbon, highly hydrophobic, adsorbs rapidly BSA. The spontaneous desorption of the protein was studied by increasing the ionic force of the medium, and also by direct adsorption competition by adding detergents like tween ${ }^{\circledR}$ or triton ${ }^{\circledR}$. The percentage of BSA that desorbs from the surface of the particles was minimum even at the strongest conditions of desorption. After the treatment with PMAOPEG the adsorption of BSA under the same conditions is practically null. 


\section{Chapter 5}

\section{In-vitro experiments}

This chapter comprises the studies of biocompatibility of the synthesised particles and the uptake by living cells in culture. The interaction of $\mathrm{Fe@C}$ particles with blood cells and platelets and the main haematological and rheological parameters have been studied to check the biocompatibility of circulating particles in blood.

The internalisation of $\mathrm{Fe} @ \mathrm{C}$ particles into dendritic cells (DCs) has been investigated to asses the final location of the particles and the viability of the resulting cell culture. Magnetic measurements of the magnetically loaded cells allow the detection of the nanoparticles into DCs, as well as their quantification. Fe@C particles were chosen because of their high saturation moment and high Curie temperature, in order to minimise the required clinical dose due to toxicity effects. Dendritic cells were able to incorporate magnetic nanoparticles in a range of size from few nanometers to about $200 \mathrm{~nm}$, after $24 \mathrm{~h}$ of culture. The actual uptake of the magnetic particles by the DCs has been demonstrated directly from the transmission electron microscopy images. TEM images proved that the particles are located mainly in the lysosomes. Carbon encapsulated iron particles showed no toxic effects on the DCs and had no apparent effect in their viability.

Finally, the physical adsorption and release of the chemotherapy agent doxorubicin on three types of particles: Fe@C produced by the discharge-arc 
method, $\mathrm{Fe}_{3} \mathrm{O}_{4} @ \mathrm{SiO}_{2}$ synthesised by the sol-gel method and $\mathrm{Fe}_{3} \mathrm{O}_{4} @ \mathrm{ZY}$ obtained by mechanical milling, have been studied in-vitro. The drug spontaneously adsorbs on the surface of the particles and is released over time in a simulated experimental model of human plasma at $37^{\circ} \mathrm{C}$. Even though these are non-porous materials, the high sorption capacity of both carbon and silica allows them to load a significant amount of the cytotoxic medicine. The kinetics of adsorption and desorption of doxorubicin was followed by vis-uv spectrophotometry.

\subsection{Biocompatibility}

To test the biocompatibility of the particles in contact with circulating blood a series of "in vitro" and "in vivo" haematological and rheological studies were performed. The study of these hemorheological parameters is usually recommended for: biocompatibility [Nageswari1998], conventional radiological contrast media [Reinhart2005], and nanoparticle magnetic resonance imaging [Lind2002] testing. These protocols were followed and adapted to nanoparticles in the blood stream. Nanoparticles are similar in shape and length to large circulating plasma proteins, such as fibrinogen or immunoglobulins. As these proteins have a decisive influence on plasma and blood viscosity, it seems important to study the possible effect of nanoparticles on these two parameters. Moreover, another important parameter includes the possible increase of blood erythrocyte aggregation, which could have a dramatic effect on the micro-vessel circulation.

To prepare the biocompatible suspensions, 100 mg of Fe@C nanoparticles produced by the arc-discharge method were suspended in $100 \mathrm{ml}$ of Gelafundine ${ }^{\circ}$, a commercial succinylated gel commonly used as a substitute of blood plasma. The solution was then sonicated for fifteen minutes. Human blood from 5 healthy volunteer donors was tested in vitro, before and after being mixed with the particles suspension in different dilutions ( $1 \mathrm{ml}$ of blood and $0.06 ; 0.12 ; 0.24$ and $0.5 \mathrm{ml}$ of 
particles). The same procedure was followed for the tests in animals (ex-vivo tests): 10 non-injected New Zealand rabbits (as control) and other 10 animals (test rabbits) after intravenous injection of $1 \mathrm{ml}$ of the particles suspension in Gelafundine ${ }^{\circledR}$. Blood samples were taken $10^{\prime}, 30^{\prime}$ and 24 hours after the injection of particles.

A complete study of human blood coagulation and rheology in-vitro has been performed (see Fig. 5.1 and Table 5.1). All coagulation tests before and after mixing human blood with different concentrations of nanoparticles were in the normal range of our laboratory. D-Dimer levels over 200 micrograms/liter were not detected. Higher levels would be expected in case of activation of the coagulation system. There are neither statistical, nor clinical significant differences between control blood and blood with different concentrations of particles.

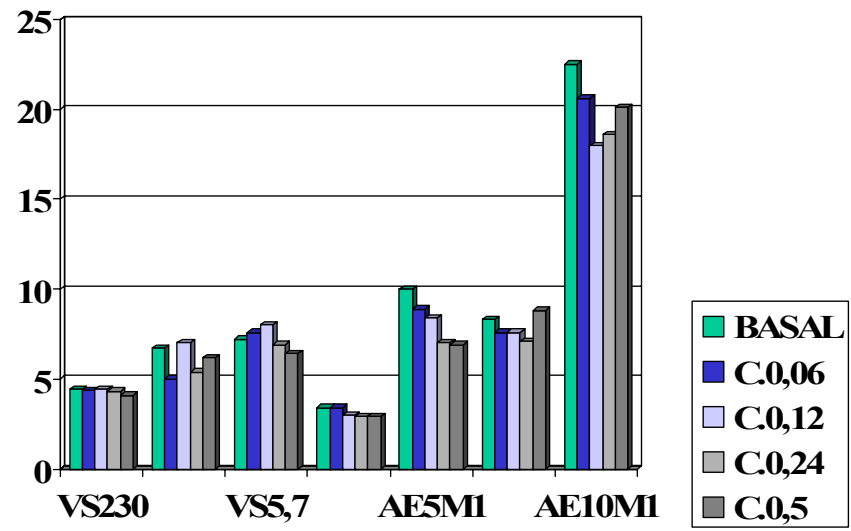

Figure 5.1: Blood Viscosity at different shear rates. $\mathrm{AE}=$ Erythrocyte aggregation $(\mathrm{M}$ : stasis, $\mathrm{M} 1$ minutes under low shear rate). $\mathrm{VS}=$ Blood viscosity Shear rate $\left(\mathrm{s}^{-1}\right) . \mathrm{C}=$ Particles concentration $(\mathrm{mg} / \mathrm{ml})$

Concerning the ex-vivo tests with rabbits' blood samples, there are no significant differences between blood samples taken up at different times after the 
injection of particles.

PT (Prothrombin time) and APTT (Activated Partial Thromboplastin Time) are slightly longer in test rabbits than in controls but the difference has no statistical significance (see Table 5.2). From the clinical point of view, some test rabbits are in a very mild hypo-coagulation state that does not exceed the recommended value for prevention of thrombosis during surgery. PT and APTT experimental/control ratios are 1.35 and 1.15 respectively. The recommended ratios in humans for prophylaxis of thrombosis range from 2.0 to 3.0. The dispersant may contribute in part to this mild hypo-coagulation state in test rabbits, which is still within the tolerable limits. Fibrinogen is moderately elevated in test rabbits, as expected, because fibrinogen is an acute-phase reactant. Nevertheless the slight rise in fibrinogen is in the upper-normal range; it is within the tolerable limits and can be attributed to the stress produced for invasive procedures. Hemoglobin, erythrocytes, leukocytes (including differential count) and platelets are in the normal range both before and after the intravenous administration of particles. There are neither statistical, nor clinical significant differences.

All previous data show good biological compatibility in this first approach. The laboratory tests applied in this experiment are the same as those used in human pathology in order to detect significant clinical disturbances in circulating cells, coagulation and rheology. Mild adverse effects can be detected with more sophisticated coagulation tests that shall be done in a second step: aggregation of platelets, prothrombin fragment $1+2$ and thrombin-antithrombin complex. Platelets aggregates have not been detected either by the cell-counter or by microscopic examination. However, we have not explored a possible inhibition of platelet aggregation. This can be done with different inductors: thrombin, epinephrine or collagen. Prothrombin fragment $1+2$ and thrombin-antithrombin complexes detect minimal activation of coagulation, but there are not routine tests and they are 
usually reserved for research purposes in human pathology [Iturbe1999]. The idea is to adapt these techniques to rabbit blood and explore them in a second step.

Table 5.1: Mean values of blood viscosity (centipoises) in five Fe@C samples. BV $=$ Blood Viscosity at different shear rates (s-1); EA = erythrocyte aggregation index; EA $5 / 10 \mathrm{M}=$ integration time at 5 and $10 \mathrm{~s}$ of stasis, conventional units; EA 5/10 M1 = integration time at 5 and $19 \mathrm{~s}$ at very low shear rate.

\begin{tabular}{|c|c|c|c|c|c|}
\hline & $\begin{array}{c}\text { Human } \\
\text { Basal }\end{array}$ & $\begin{array}{c}\text { Human } \\
0.06 \mathrm{~cm} 3\end{array}$ & $\begin{array}{c}\text { Human } \\
0.12 \mathrm{~cm} 3\end{array}$ & $\begin{array}{c}\text { Human } \\
0.24 \mathrm{~cm} 3\end{array}$ & $\begin{array}{l}\text { Human } \\
0.5 \mathrm{~cm} 3\end{array}$ \\
\hline BV $230 \mathrm{~s}^{-1}$ & 4.44 & 4.36 & 4.45 & 4.33 & 4.1 \\
\hline BV $23 s^{-1}$ & 6.7 & 5.0 & 7 & 5.4 & 6.2 \\
\hline BV $5.7 \mathrm{~s}^{-1}$ & 7.2 & 7.6 & 8 & 6.8 & 6.4 \\
\hline EA $5 \mathrm{M}$ & 3.4 & 3.4 & 3 & 2.9 & 2.9 \\
\hline EA 5M1 & 10 & 8.9 & 8.4 & 7 & 6.9 \\
\hline EA $10 \mathrm{M}$ & 8.3 & 7.6 & 7.6 & 7.1 & 8.8 \\
\hline EA 10M1 & 22.5 & 20.6 & 18 & 18.6 & 20.1 \\
\hline
\end{tabular}

Table 5. 2: $\mathrm{PT}=$ prothrombin time (s); APTT = activated partial thromboplastin time (s); D-Dimer $(\mu \mathrm{g} / \mathrm{l})$.

\begin{tabular}{lccc}
\hline & Controls $\chi \pm$ SD & Experimental $\chi^{ \pm \text {SD }}$ & P (Mann-Winley) \\
\hline PT & $7.067 \pm 0.071$ & $9.6 \pm 2.5$ & 0.08 \\
APTT & $57.025 \pm 26.4$ & $65.920 \pm 30$ & 0.253 \\
Fibrinogen & $248 \pm 149$ & $381.8 \pm 85.5$ & 0.0388 \\
D-Dimer & $<200$ & $<200$ & \\
\hline
\end{tabular}




\subsection{Cell uptake of nanoparticles}

Dendritic Cells (DCs) are the main antigen presenting cells (APCs) of the immune system. Their main features are the potential to capture antigens, to process several kinds of antigens and their ability to migrate from peripheral tissues to secondary lymphoid organs for presenting these antigens to $T$ cells [Steinman1991, Hart1997, Banchereau1998]. The presence of dendritic cells has been identified in various types of tumours: like head and neck tumours, breast cancer, cervical or endometrial cancer [Coppola1998], gastric cancer [Kakeji1993] or renal cancer [Thurnher1996]. Clinical pathologists have also associated the presence of a great number of DCs with a better prognosis [Ambe1989, Furihata1992]. It is believed that DCs incorporate into the epithelial tissue of a growing tumour, therefore the inclusion of particles inside dendritic cells would play the part of a "Horse of Troy", so as to stealthily reach the tumour site and there perform its function, whether it is the release of a chemotherapy agent, act as a contrast agent or selectively heat a specific area by applying an alternate magnetic field. With this purpose, the inclusion of Fe@C particles inside DCs has been studied by means of magnetic measurements to check the final location of the particles and the viability of the resulting cell culture.

Dendritic cells are present in small quantities in tissues that are in contact with the external environment, mainly in the peripheral tissues. They can also be found at an immature state in the blood. Once activated, they migrate to the lymphoid tissues where they interact with $\mathrm{T}$ cells and $\mathrm{B}$ cells to initiate and shape the adaptive immune response. [Steinman1973]. DCs can be obtained from myeloid or plasmacytoid progenitors [Ito2005]. DCs obtained from myelomonocytic progenitors (MDCs) and maturated with tumour antigens have showed antitumoral activity in animal models [Mayordomo1995] and humans [Nestlé1998]. 
Activation and maturation of DCs consists of a series of morphologic, phenotypic and functional changes, characteristic of their evolution from immature to mature state [Banchereau1998]. There are several mechanisms that induce the change from one state to the other. The recognition of pathogens patrons [Reis 1996] or the alteration on the internal medium [Pulendran2004] are among them. Interaction between DCs and lymphocytes CD4+, CD8+ and Natural Killer cells (NKs) can also induce their activation and maturation [Wilson1997, Rissoan1999, Schuster2002, Fernández2003, Sousa2004].

Tissue DCs are normally in immature state. This state is characterised by high endocytosis capacity and low surface expression of human leukocyte antigen (HLA) and co-stimulation molecules, such as CD40, CD80 or CD86 [Dodaphkar2001]. Therefore immature DCs have a poorly developed antigen presentation activity. Under the influence of certain stimuli, DCs are activated and evolve to a mature state. At this stage of development they grow branched projections, the dendrites, which give the cell its name. They progressively lose the endocytosis capacity, whereas the ability to process and present antigens on surface HLA molecules is significantly increased. Maturation of DCs involves changes that allow them to migrate to secondary lymphoid organs as well. Here they are able to interact with naive lymphocytes [Foti1999, Hopken2004]. The effectiveness of the interaction DC-T cell depends, amongst others, on the expression of the surface costimulatory molecules as CD80 and CD86 [Hart1993, Symington1993, McLellan1995].

First step of every specific immune response mediated by $\mathrm{T}$ lymphocytes consists on the reception of antigens by the APCs. Alive foreign cells or cells from the own organism, cells that have gone through necrosis or apoptosis, cells infected with viruses or parasites, bacteria, immunocomplexes and every kind of molecule that is in the medium surrounding the APC can be detected and considered as 
antigens. Its structure and functional design allows the DC to endocyte the foreign molecule by different mechanisms: macropinocytosis, phagocytosis or receptormediated endocytosis. In pinocytosis, molecules are transported inside the cell, forming small vesicles that subsequently fuse with lysosomes. Unlike phagocytosis or receptor-mediated endocytosis, pinocytosis is non-specific in the molecules internalised by the cell. Macropinocytosis is DCs' main mechanism of internalisation, since it allows the cell to internalise the highest and more varied amount of antigens [Sallusto1995]. After the internalisation of the antigen, the dendritic cell increases its capacity to perform biochemical processes that mediate the intracellular processing of the internalised material and their charge over HLA molecules [Mellman2001], starting that way the immunologic response.

Dendritic cells can also have an important role in angiogenesis mechanisms irrigating tumour cells. Angiogenesis is a physiological process involving the growth of new blood vessels from pre-existing vessels. It is a normal process in growth and development, as well as in wound healing. However, this is also a fundamental step in the transition of tumours from a dormant state to a malignant state [Prior2004]. Tumours with less than $2 \mathrm{~mm}$ of diameter allow diffusion transport among their cells, however bigger tumours need a network of vessels to irrigate all the cells. On the other hand, VEGF (Vascular Endothelial Growth Factor) has been demonstrated to be a major contributor to angiogenesis, increasing the number of capillaries in a given network. Initial in vitro studies demonstrated that bovine capillary endothelial cells will proliferate and show signs of tube structures upon stimulation by VEGF. Recently the induction of endothelial cell features in MDCs cultivated with angiogenic factors like VEGF has been demonstrated [Conejo2005] and similar cells have been isolated from human tumour vessels [Conejo2005], opening the possibility of targeting MDCs with incorporated magnetic nanoparticles to tumours and use them to visualise tumours or in oncology therapy. 
The mechanism by which a given cell type recognises and incorporates a passive nanoparticle is not well understood yet. Current improvements in this field rely on new functionalisation processes of the particles with specific ligands for targeting specific cell membrane receptors [Jaulin2000]. In any case, any particulate system to be used as targeting carrier should mimic biological units in order to pass through the reticuloendothelial system (RES) without being detected. Tumour targeting with nanoparticle-loaded DCs is a potentially interesting strategy because no RES activity is expected for the magnetic carrier, since DCs from the same patient would pass unnoticed as a part of the same immune system.

Several previous studies have reported on the uptake of different complexes by the cell, or the inclusion of latex-type nanospheres [Wilhelm2003, Coester2006], proposing models for the mechanisms of phagocytosis as well [Christine2002]. However, only recently some works [Anderson2005, De Vries2005] have been devoted to the specific case of magnetic nanoparticles, composed of metallic and ceramic phases. The magnetic performance of the particles is usually enhanced if materials with large saturation magnetisation values are chosen, such as $\mathrm{Fe}$, Ni or $\mathrm{Fe}_{3} \mathrm{O}_{4}$. One of the key issues that restricts the choice of the magnetic material is related to the toxicity effects that many of these elements (or their oxides) have on the cellular metabolism.

Following informed consent, mononuclear cells obtained from $14 \mathrm{ml}$ of blood of healthy volunteers were cultured during seven days with AIM-V media with Interleukin $4\left(10 \mathrm{U}^{5} / 100 \mathrm{ml}\right)$ and Granulocyte-macrophage colony stimulation factor $\left(10 \mathrm{U}^{5} / 100 \mathrm{ml}\right)$. On day five, $5 \mu \mathrm{g} / \mathrm{ml}$ of media containing lypopolysacharides were added to induce maturation of DCs, which was confirmed by flow cytometry on an aliquot of cultured cells on day 6 . On day $6,15 \mu \mathrm{l} / \mathrm{ml}$ of media of a $5 \%$ suspension of iron-carbon (Fe-C) nanoparticles were added to the culture. Finally, cells were collected on day 7, washed twice with PBS and resuspended in the same buffer and 
centrifuged during $25 \mathrm{~min}$ at $400 \mathrm{G}$ with Ficoll histopaque $(1.077 \mathrm{~g} / \mathrm{ml})$. DCs were isolated from the PBS/Ficoll interfase and unincorporated NPs remained in the bottom of the tube. Incorporation of MNP was assessed by confocal microscopy (data not shown) and transmission electron microscopy. For the latter, pelleted cells were fixed in PBS containing 2\% glutaraldehyde at room temperature for 30 minutes and kept overnight at $4^{\circ} \mathrm{C}$. The cells were then washed three times with PBS and treated with $1 \% \mathrm{OsO}_{4}$ in PBS for 10 minutes. All of the samples were washed three times in $\mathrm{H}_{2} \mathrm{O}$ dehydrated in an ascending series of ethanol solutions. Propyleneoxide (PO) was used as a transitional solvent. The cells were incubated overnight in a solution containing a 1:1 mixture of PO and Epon-Araldite (EA) (Electron Microscopy Sciences). The next day, the mixture was replaced with $100 \%$ EA, and the capsule was placed in a desicator for 8 hours. The sample was then placed in plastic capsules containing EA and polymerised at $60^{\circ} \mathrm{C}$ for 48 hours. Thin $(0.1 \mu \mathrm{m})$ sections were cut using a microtome and placed on 200 mesh $\mathrm{Cu}$ grids. The samples were stained with $1 \%$ aqueous uranyl acetate and Reynolds lead citrate. Sections were examined with a Hitachi 7100 transmission electron microscope (Hitachi High Technologies).

Dendritic cells were cultured in a 12-well plate, and NPs incorporated as described above. Cells were collected from different plates on days 7, 8, 9, 10 and 11 and viability of the DCs was assessed by the exclusion of trypan blue.

For magnetic measurements, all samples were conditioned in closed containers before quenching the DCs/MNPs mixture below its freezing point $(\sim 265 \mathrm{~K})$ from room temperature. A commercial SQUID magnetometer was used to perform static measurements as a function field and temperature. Magnetisation data was collected in applied magnetic fields up to $1 \mathrm{~T}$, between $5 \mathrm{~K}$ and $250 \mathrm{~K}$ to avoid the melting of the solid matrix (solvent). 
The incorporation of NPs onto CDs was assessed by electron microscopy. The nanoparticles are visible as aggregates in lysosomes (Fig 5.2).
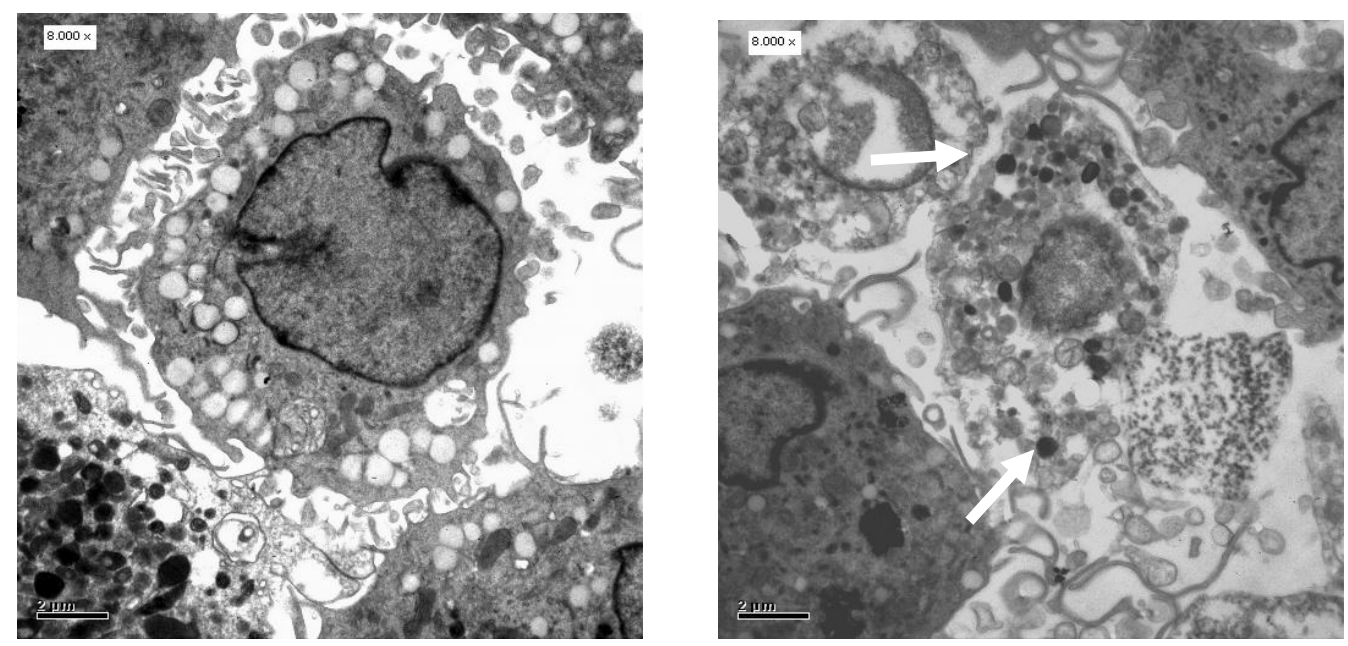

Fig. 5.2. Electron microphotographs of DCs without (A) and with (B) Fe@C particles. Intralysosomal particles aggregates are shown by a white arrow.

Regarding viability after the co-incubation of dendritic cells with the magnetic particles suspension, the fraction of viable cells on days 7, 8, 9, 10 and 11 was not significantly affected by the incorporation of the nanoparticles (Fig. 5.3).

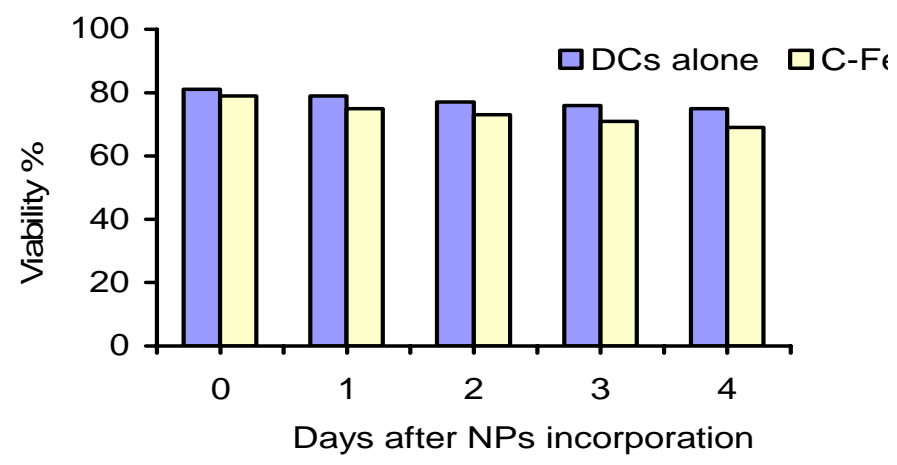

Fig. 5.3: Fraction of viable cells after the incorporation of Fe@C nanoparticles compared with untreated DCs 
After the centrifugation of loaded cells in a density gradient, all the magnetic particles not included into the DCs are located in the pellet, whereas the floating band is composed of DCs loaded with magnetic particles, with eventual small fractions of non-loaded cells. Magnetism was measured for both the material from the band (dendritic cells with magnetic particles), and from the pellet (magnetic particles only).
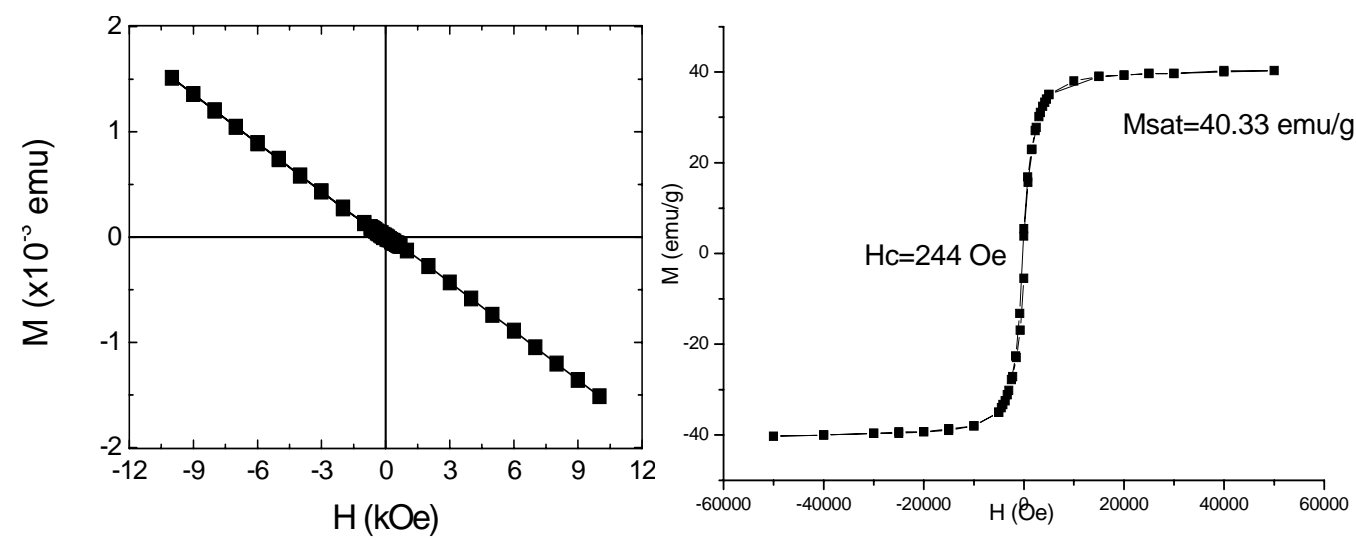

Figure 5.4: a) Magnetic response of the dendritic cells culture. The linear response with a negative slope is significant of a diamagnetic behaviour. b) Hysteresis loop of the Fe@C particles.

Figure 5.4 compares the magnetic response from the initial DCs, before loading the particles, with the original curve for the isolated magnetic particles. For DCs alone, the negative response to positive applied fields indicates that the overall signal is diamagnetic. For the loaded cells a hysteresis loop is obtained (Fig. 5.5), due to the contribution of the internalised particles (Fig 5.4 b). This ferromagnetic component is superimposed to the (small) diamagnetic signal from the DCs. This can be deduced from the negative slope of the $\mathrm{M}(\mathrm{H})$ curves at high fields. After normalising both measurements to the total culture volume, the diamagnetic signal of the cells can be subtracted from the total system signal to obtain a net magnetic 
signal, which shows the expected nearly saturation behaviour for a ferromagnetic system, as in the case of the Fe@C particles (Fig. 5.5).

Identical magnetic parameters of both the initial $\mathrm{Fe} @ \mathrm{C}$ particles and magnetically loaded DCs were obtained, indicating that the particle size distribution remains unaltered after the incorporation into DCs. The endocytosis process is then not size-selective within the size range of Fe@C particles: between 10-200 $\mathrm{nm}$. Also, the amount of magnetic material incorporated by the DCs yielded a magnetic signal $\left(10^{-3} \mathrm{emu}\right)$ that is larger than the diamagnetic signal from biological tissues $\left(10^{-4}-10^{-5} \mathrm{emu}\right.$ for DCs at low fields), and quite above the detection limit of SQUID measurements $\left(10^{-7} \mathrm{emu}\right)$. This situation makes the separation process potentially powerful for the detection of small amounts of all types of targeted cells as, for example, the case of metastatic cells within the blood stream or lymph nodes.

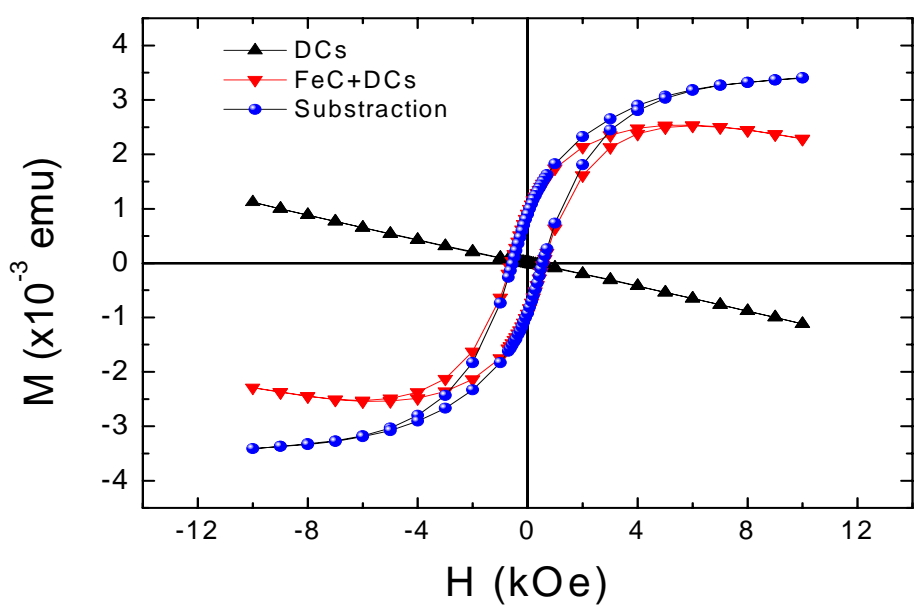

Fig. 5.5 Magnetic Response from Culture DCs incubated with Fe@C particles. The diamagnetic signal of the cells can be subtracted from the total system signal to obtain a net magnetic signal, which shows the expected nearly saturation behaviour for a ferromagnetic system. 


\subsection{Drug adsorption and release studies}

The capability of the particles to spontaneously adsorb and release a drug has been tested for the $\mathrm{Fe} @ \mathrm{C}, \mathrm{Fe}_{3} \mathrm{O}_{4} @ \mathrm{ZY}$ and $\mathrm{Fe}_{3} \mathrm{O}_{4} @ \mathrm{SiO}_{2}$ samples. Kinetics of adsorption was measured following the procedure described by Kuznetsov [Kuznetsov1999]: A $100 \mu \mathrm{g} / \mathrm{ml}$ solution of doxorubicin hydrochloride was mixed with $1 \mathrm{mg} / \mathrm{ml}$ of the particles in distilled water. The suspension was incubated on a shaker at room temperature, and samples were taken after fixed intervals of time. The adsorbent magnetic phase was sedimented with a 3 KOe permanent magnet, and the optical density of the supernatant measured with a UV spectrophotometer at $498 \mathrm{~nm}$ (Fig. 5.6). A calibration curve is previously done for known concentrations of the drug, then the concentration of the adsorbed drug can be directly calculated making an extrapolation from the curve. Experimental details are described in section 2.4.3 (page 69).
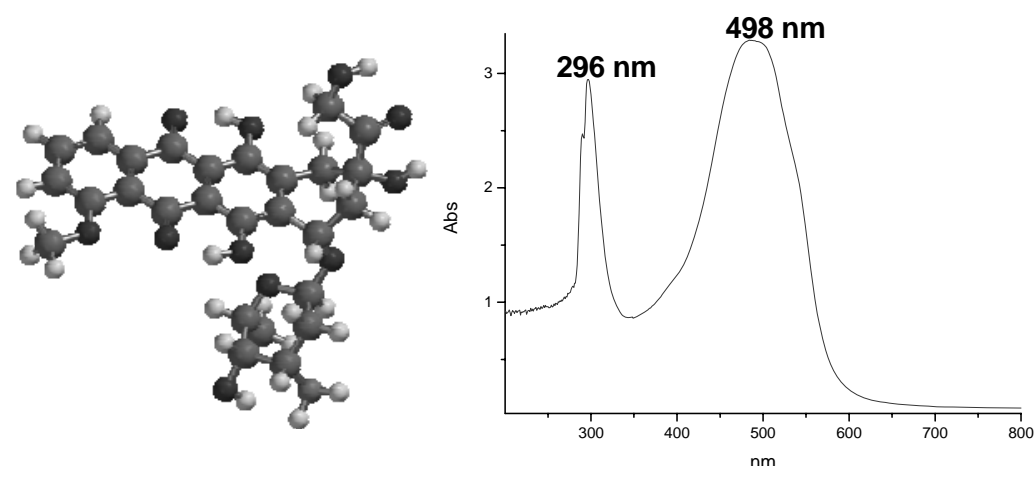

Figure 5.6. 3D representation of the molecule of doxorubicin. This compound absorbs visible light at 498 and $296 \mathrm{~nm}$.

Desorption kinetics was studied trying to simulate in-vivo conditions. $5 \mathrm{mg}$ of the particles, previously incubated in a solution of doxorubicin, were suspended in $5 \mathrm{ml}$ of real human plasma, and warmed at $36^{\circ} \mathrm{C}$ while gently stirring. The magnetic phase was sedimented with a magnet, and the optical density of the 
supernatant measured with a UV spectrophotometer at $296 \mathrm{~nm}$ every $2 \mathrm{~h}$ intervals during the first day and every $8 \mathrm{~h}$ thereafter. The particles slowly release the drug, and saturation is reached after 3 days. Figure 5.7 shows the scheme for the incubation of particles with doxorubicin, for the adsorption of the drug and its release in human plasma. Figure 5.8 is an example of the kinetics of adsorption and release of the drug from the surface of the particles, in this case from the $\mathrm{Fe}_{3} \mathrm{O}_{4} @ \mathrm{ZY}$ samples. Doxorubicin is released from the surface of the particles at a slow enough rate to reach the affected organ with the permanent magnet and efficiently treat the tumour.

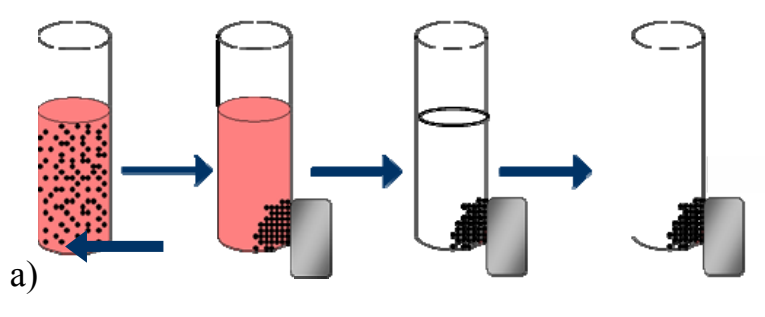

b)

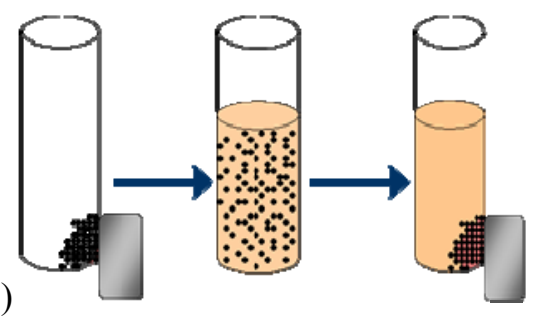

Figure 5.7: a) Diagram of the incubation of drug and nanoparticles. Particles are suspended in a doxorubicin solution. At fixed intervals of time particles are separated with a magnet and the absorbance of the supernatant is measured, then the magnet is removed and suspension stirred again. After reaching the maximum adsorption, the liquid is decanted. $b$ ) The particles, loaded with the drug, are again suspended in a human plasma solution and incubated at $37^{\circ} \mathrm{C}$. Again, at fixed intervals of time particles are separated and the absorbance of the supernatant measured to obtain the kinetics of adsorption and release. 

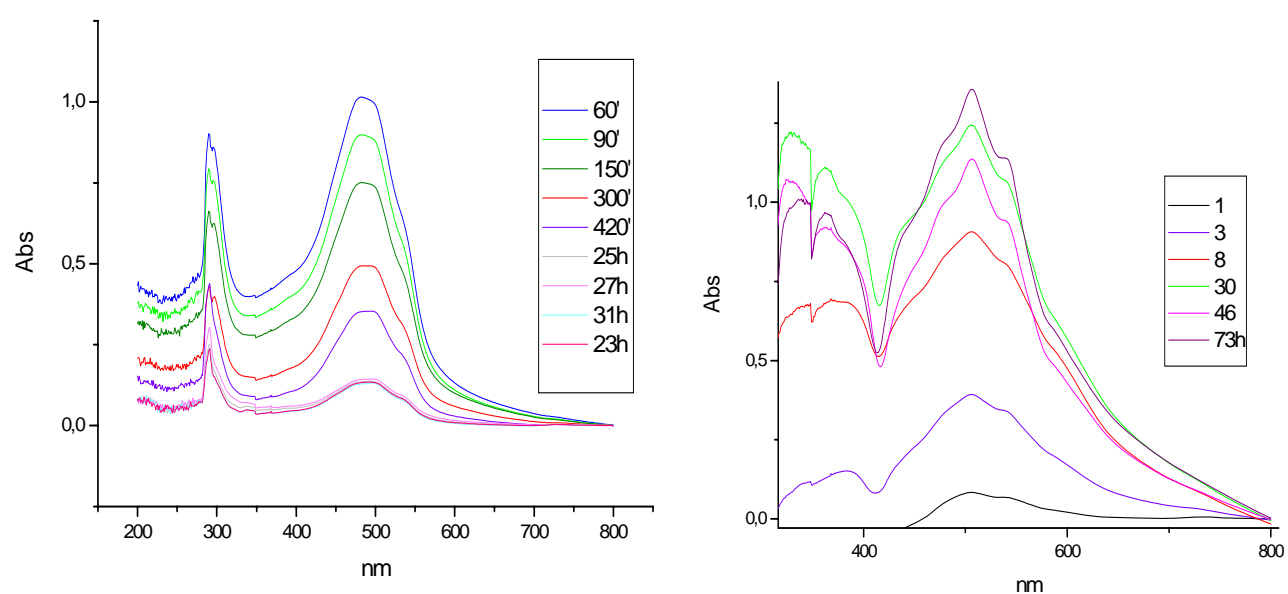

Figure 5.8: Absorbance spectra of the adsorption and desorption of doxorubicin on the $\mathrm{Fe}_{3} \mathrm{O}_{4} @ Z \mathrm{ZY}$ particles.

There are several kinetics models to describe drug dissolution or release profiles [Costa2001]. These theoretical models account for the amount of drug dissolved from the pharmaceutical dosage system as a function of time. The quantitative interpretation of the values obtained in the dissolution assay is facilitated by the use of a generic equation that mathematically translates the dissolution curve in function of some parameters related with the pharmaceutical dosage forms. In some cases, the fit equation can be deduced by a theoretical analysis of the process, as in the case of the zero order models. In most cases, however, theoretical fundament is very complex and more adequate empirical formalisms are required. Also, the intrinsic nature of the drug crystallinity, particle size or solubility can affect the kinetics of release [El-Arini1995]

The release models with major acceptance and best describing drug release phenomena are the zero order models, the Higuchi model and the KorsmeyerPeppas model [Costa2001]. The zero order models describe the case in which the drug release kinetics follows a linear response: 


$$
f_{t}=k t
$$

Where $f_{t}$ is a function of time related to the amount of drug released from the particles. This equation becomes ideal to describe coated dosage forms or membrane controlled dosage forms.

The Higuchi model has a large application in polymeric matrix systems. Higuchi developed several theoretical models to study the release of water soluble and low soluble drugs incorporated in semi-solid and solid matrixes [Higuchi1961, Higuchi1963]. Mathematical expressions were obtained for drug particles dispersed in a uniform matrix which behaves as the diffusion media. One of these models describes the drug release from spherical homogeneous matrix systems and planar or spherical systems with a granular or heterogeneous matrix.

Higuchi power law equation $f_{t}=k_{H} t^{1 / 2}$

Where $k_{H}$ is the Higuchi dissolution constant, treated in a different manner by different authors and theories. Higuchi describes drug release as a process based in Fick's law, square root time dependent. This relation can be used to describe the drug dissolution form several types of modified release pharamaceutical dosage forms, as in the case of some transdermal systems [Costa1996] and matrix tablets with water soluble drugs [Desai1966, Scwhartz1968].

The Higuchi and the zero order model represent two limit cases of drug transport and release, whereas the Korsmeyer-Peppas can be useful to describe an intermediate situation between both models. This is a semiempirical model that relates the drug released to the elapsed time exponentially:

Korsmeyer-Peppas model: $f_{t}=a t^{n}$ 
Where $a$ is a constant that incorporates structural and geometrical characteristics of the drug dosage form, $n$ is the release exponent, indicative of the drug release mechanism, and the function of $t$ is $\mathrm{M}_{t} / \mathrm{M}_{\infty}$, fractional release of drug.

A water soluble drug incorporated into, or adsorbed onto a matrix, is mainly released by diffusion. This is the case of doxorubicin from the surface of the magnetic nanoparticles. Temperature is increased from room temperature for adsorption to $37^{\circ} \mathrm{C}$ of the release experiment; two possible effects govern then the diffusion of doxorubicin out of particles: increasing hydrophobicity of the matrix, which should slow down the release, and increasing mobility of the molecule, with the opposite effect [Missirlis2006].

Table 5.3: Comparison of the adsorption and release capability for different materials

\begin{tabular}{ccccc}
\hline Particle & $\begin{array}{c}\text { C doxo } \\
\text { adsorption }\end{array}$ & $\begin{array}{c}\text { Time of } \\
\text { adsorption } \\
\text { (saturation) }\end{array}$ & $\begin{array}{c}\text { C doxo } \\
\text { desorption }\end{array}$ & $\begin{array}{c}\text { Time of } \\
\text { desorption }\end{array}$ \\
\hline $\mathrm{FeC}$ & $71 \mathrm{ppm}$ & $3 \mathrm{~h}$ & $60 \mathrm{ppm}$ & $72 \mathrm{~h}$ \\
$\mathrm{Fe}_{3} \mathrm{O}_{4}-\mathrm{ZY}$ & $168 \mathrm{ppm}$ & $8 \mathrm{~h}$ & $100 \mathrm{ppm}$ & $75 \mathrm{~h}$ \\
$\mathrm{Fe}_{3} \mathrm{O}_{4}-\mathrm{SiO}_{2}$ & $107 \mathrm{ppm}$ & $24 \mathrm{~h}$ & $70 \mathrm{ppm}$ & $80 \mathrm{~h}$ \\
\hline
\end{tabular}

Table 5.3 is a comparison of the loading capacities and kinetics of adsorption and release of the three studied materials. Maximum adsorption was achieved for the magnetite-zeolite composites, up to $168 \mathrm{ppm}$ after $8 \mathrm{~h}$ of incubation, increasing to $186 \mathrm{ppm}$ after $23 \mathrm{~h}$ of incubation. The adsorption for the magnetite-silica nanoparticles is also high, $107 \mathrm{ppm}$, whereas the lowest adsorption is found the carbonous particles. This can be explained in terms of porosity of the materials, since silica is more porous than graphite, so its capacity of loading a drug is higher. Moreover, the BET adsorption and desorption surface areas of the iron-carbon 
particles are also much smaller than silica and zeolite coated particles, as low as 35 $\mathrm{m}^{2} / \mathrm{g}$ and $14 \mathrm{~m}^{2} / \mathrm{g}$ respectively. Nevertheless, lower values of the specific surface area have proved to be effective for a faster desorption [Cerri2004]. Therefore these particles still seem suitable for being used as drug delivery vehicles. The use of activated carbon from the first step of the synthesis could increase the specific surface area, improving that way the capability of the system to adsorb chemotherapy agents.

Doxorubicin adsorption and desorption profiles for the $\mathrm{Fe}_{3} \mathrm{O}_{4}-\mathrm{ZY}$ composites are shown in figures 5.8 and 5.9. Doxorubicin molecular dimensions are $1.27 \mathrm{x}$ $1.16 \mathrm{~nm}$ (calculated using molecular modeling; Spartan 5.0, Fig. 5.6) which are larger than the mean pore size of the obtained coated magnetic nanoparticles $(0.87$ $\mathrm{nm})$. Therefore most of the adsorption would be limited to the external surface of the nanocomposite. The BET surface area of the composite material is $442.9 \mathrm{~m}^{2} / \mathrm{g}$. It is important to point out that the BET values are given per unit of total mass $\left(\mathrm{m}^{2} / \mathrm{g}\right)$ that is, considering the total weight of both zeolite and magnetite in the composite material. $77 \%$ of the initial concentration of doxorubicin was adsorbed in $3.1 \mathrm{~h}$. Before washing to remove all the non-adsorbed doxorubicin the drug storage capacity reached was as high as $92 \%$ of the initial doxorubicin (Fig. 5.9). After washing to remove all the non-adsorbed doxorubicin and dispersing the dried drug loaded nanoparticles in human plasma at $37{ }^{\circ} \mathrm{C}$ it was observed that the drug loading capacity of the magnetic nanocomposites was the $50 \%$ of the initial doxorubicin concentration. $77 \%$ of the doxorubicin loaded on the nanoparticles was released in $13 \mathrm{~h}$, reaching saturation after $75 \mathrm{~h}$. On the other hand, final release was $84 \%$ of the initial adsorbed amount for the $\mathrm{Fe} @ \mathrm{C}$, and $65 \%$ for the $\mathrm{Fe}_{3} \mathrm{O}_{4} @ \mathrm{SiO}_{2}$, reaching saturation after 3 days. This release, associated with a diffusion controlled mechanism, is an agreement with an adsorption limited to the external surface of the nanoparticles (Fig. 5.10). Higuchi model seem a good description of both adsorption and desorption profiles. 


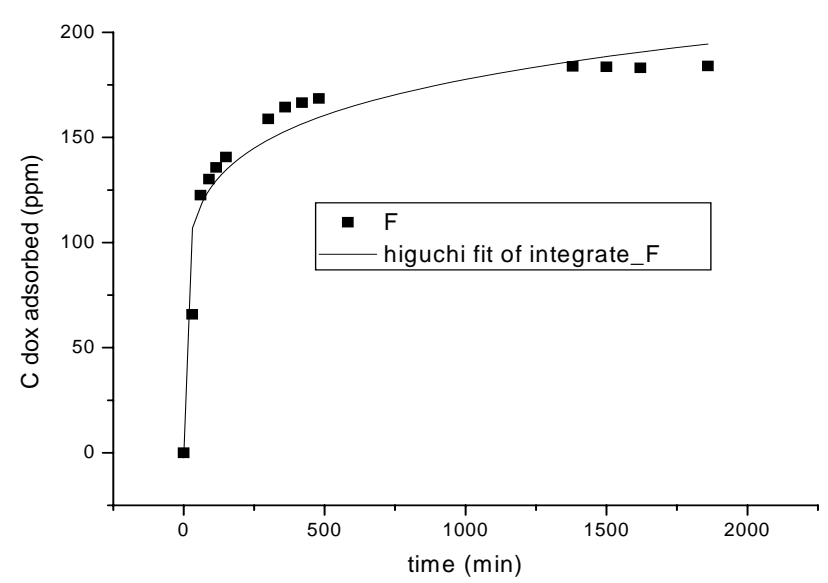

Figure 5.9: Kinetics of adsorption of doxorubicin by the $\mathrm{Fe}_{3} \mathrm{O}_{4} @ \mathrm{ZY}$ particles.

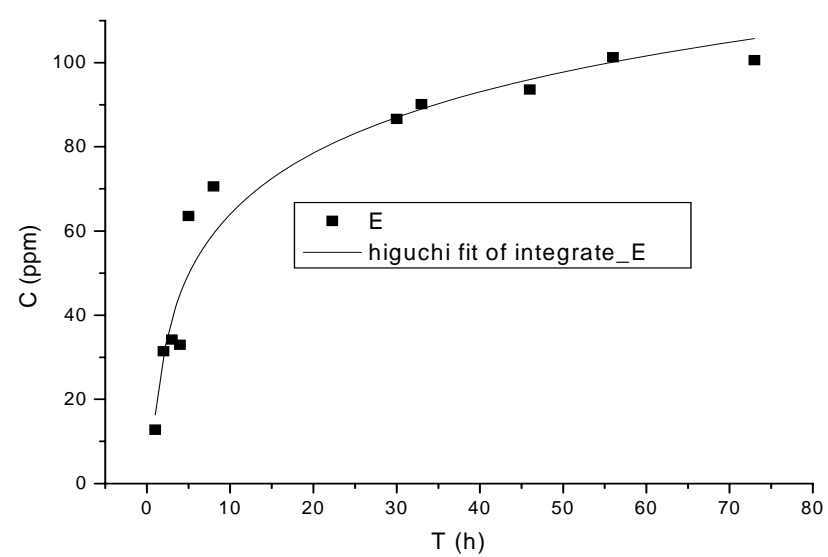

Figure 5.10: Desorption kinetics of the adsorption of doxorubicin from the $\mathrm{Fe}_{3} \mathrm{O}_{4} @ \mathrm{ZY}$ particles.

Regarding the iron at carbon particles, $39 \%$ of the initial concentration of doxorubicin was adsorbed in $3 \mathrm{~h}$, reaching saturation. This low adsorption may be due to the small surface area of the carbonous particles in comparison to the silica surfaces (Fig. 5.11). A modified Korsmeyer-Peppas model seems to be the best fit for this profile. On the other hand, release was quite fast, reaching $84 \%$ after $72 \mathrm{~h}$, in agreement with diffusion from the non-porous surface of the particles. Release 
profile fits quite well with a Higuchi model (Fig. 5.12).

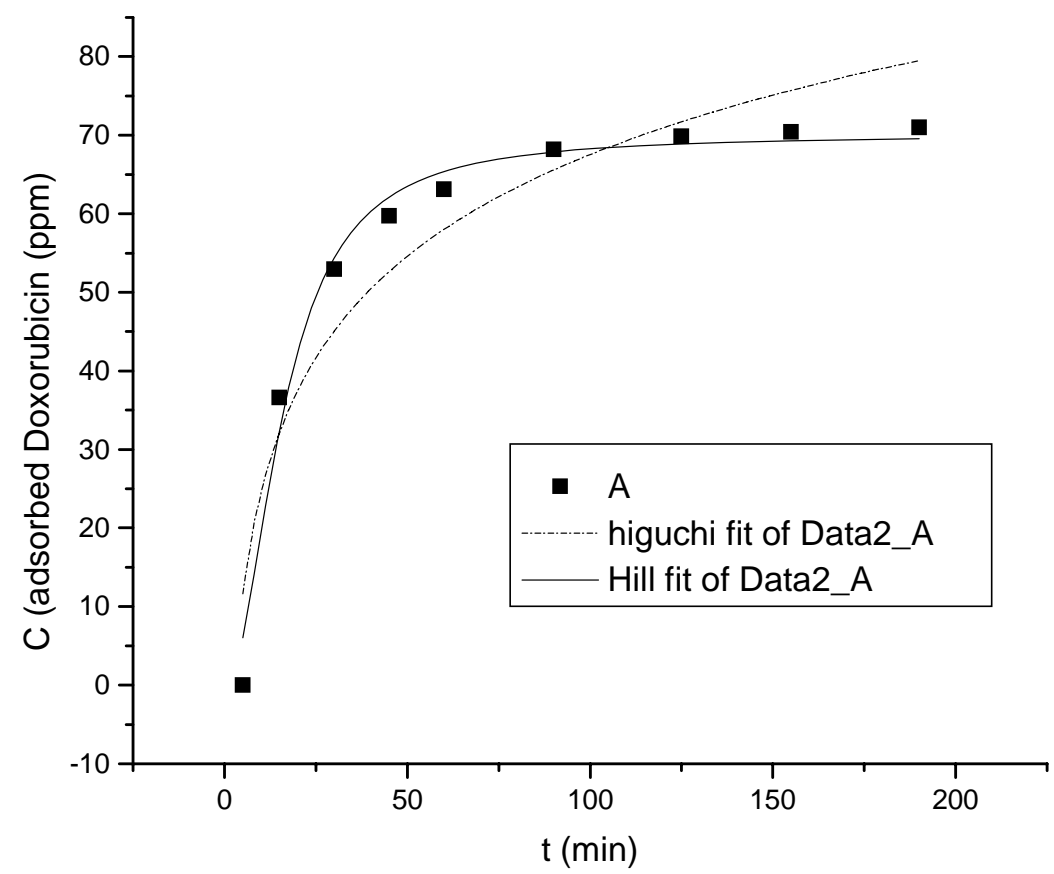

Figure 5.11: Adsorption kinetics of the adsorption of doxorubicin from the Fe@C particles. Saturation seems to be reached after three hours of incubation.

Magnetite encapsulated in silica particles had a slow adsorption profile, reaching 59\% after 24h (Fig. 5.13). Release was fast in the first hours of desorption, following a linear behaviour, reaching $37 \%$ in $60 \mathrm{~h}$. After $80 \mathrm{~h} 65 \%$ of the adsorbed doxorubicin had been released to the medium. After a fast initial release and a slower desorption at the end, the overall release profile could not be fitted to a Higuchi or Korsmeyer-Peppas model (Fig. 5.14). 


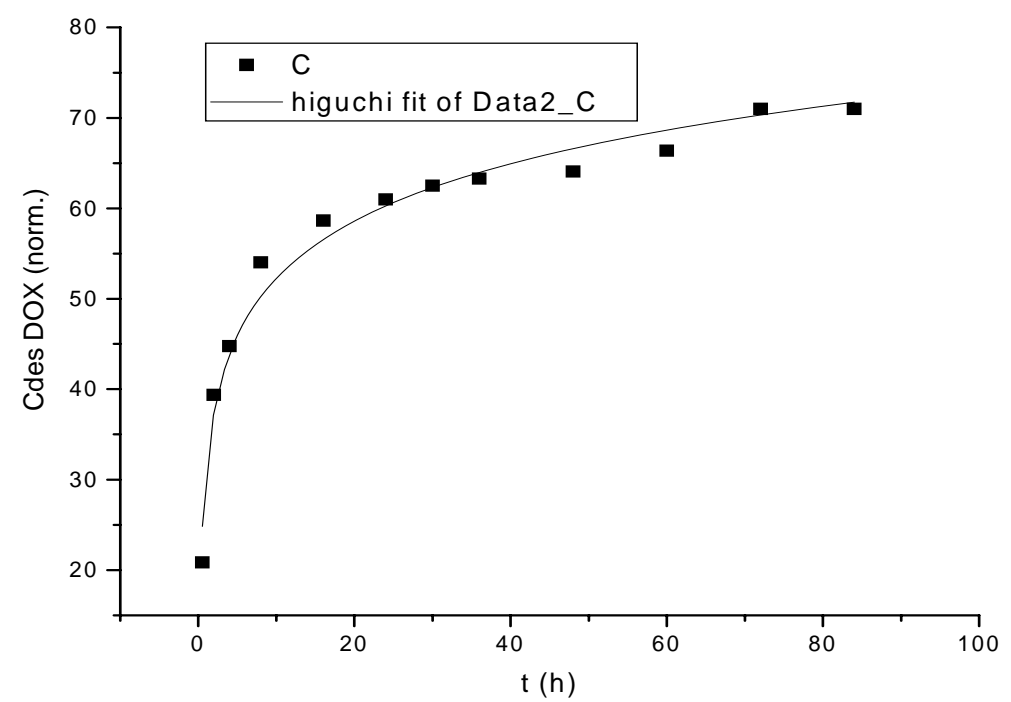

Figure 5.12: Desorption kinetics of doxorubicin from the Fe@C particles. Release is completed after three days.

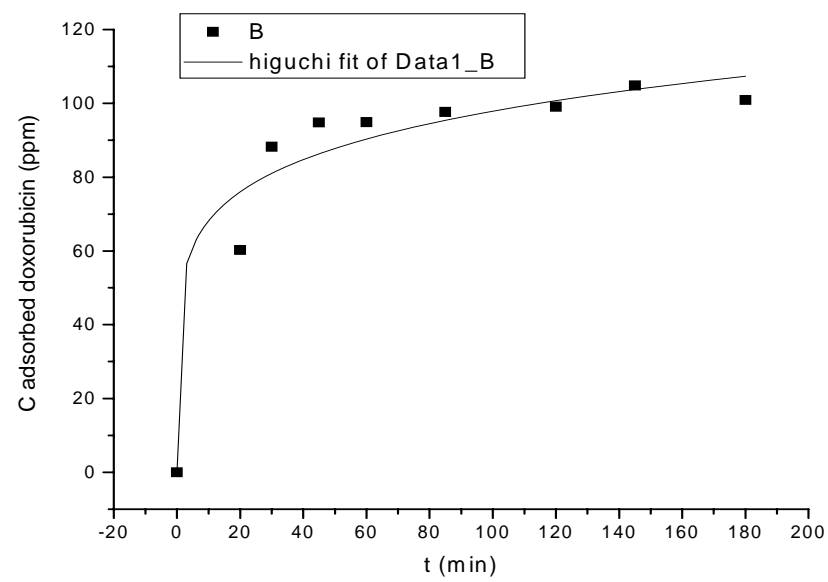

Figure 5.13: Adsorption kinetics of doxorubicin by the $\mathrm{Fe}_{3} \mathrm{O}_{4} @ \mathrm{SiO}_{2}$ particles. 


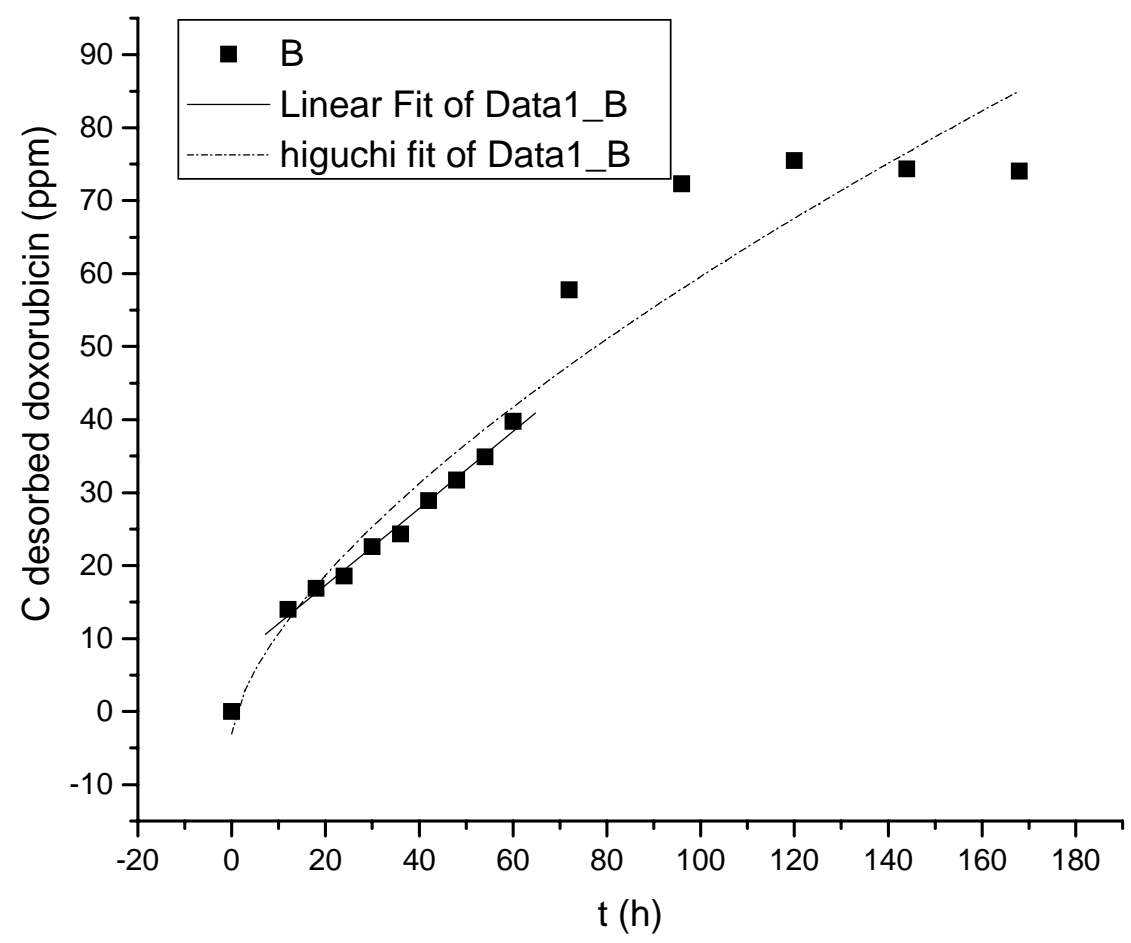

Figure 5.14: Desorption kinetics of the adsorption of doxorubicin from the $\mathrm{Fe}_{3} \mathrm{O}_{4} @ \mathrm{SiO}_{2}$ particles. Earlier set of data can be adjusted to a linear fit.

In conclusion, the three studied materials show an appreciable loading capacity of adsorption and release of the chemotherapy agent doxorubicin. Adsorption is fast and achieved within a few hours, whereas complete release takes place after several days. This controlled release would be slow enough to allow the carriers to reach the target and desorb the load only at the specific site. $\mathrm{Fe}_{3} \mathrm{O}_{4} @ \mathrm{ZY}$ has the maximum loading capacity but a faster release, related to a superficial adsorption of the drug on the surface of the particles. 



\section{Chapter 6}

\section{In-vivo results}

The distribution, behaviour and tolerance of nanoparticles in a living body after the implantation of a permanent magnet inside a deep solid organ has been thoroughly studied. Our hypothesis of work is that magnetic drug carriers injected systemically inside a living body will be attracted and retained by an internal magnetic implant. In the target area they will deliver their drug load over a prolonged time. Based on this hypothesis, a surgical experimental model of minimally invasive surgery has been developed, and it has proved to be highly efficient.

Kidney has been chosen as the target organ by several reasons: It is a double, symmetrical organ, which allows a comparative necropsy study. It has a very vascularised parenchyma, which assures a constant flow of circulating particles through it. It is situated deeply in the abdomen, but at the same time it allows easy exploration by radiology or ultrasound scan. In rabbit, whose size and characteristics are ideal for our purpose, kidneys, besides being retroperitoneal organs, can be easily visualised and approached by transperitoneal laparoscopy. This facilitates in great extent the insertion of the magnetic implant.

Moreover, the surgical team that has developed this surgical model and performs this surgery experiments is directed by an urologist with wide experience in minimally invasive surgery and urology laparoscopy. Finally, it is also important to point out that renal parenchyma tumours are, in most cases, malign and highly 
resistive to chemotherapy treatment. Nowadays, the only efficient method against them is surgical exeresis. This practice finds great difficulties when it multiple tumours or metastasis, bilateral-sited tumours, or tumours affecting organisms with only one functional kidney.

The intrarenal localisation of these tumours has to be seriously borne in mind, especially in the cases where partial nefrectomy or tumorectomy is performed. Sometimes, if tumours are placed on the hiliar or deep mesorenal zones, the total sacrifice of the organ is demanded. The general state of the patient is another circumstance to be borne in mind, as it can advice against the use of exeresis surgery.

As it is mentioned above, the efficiency of chemotherapy drugs for the treatment of renal tumours is rather low. Not all chemotherapy agents are efficient against neoplasic cells, present in tumour tissue. Most commonly used drugs, like vimblastin ${ }^{\circledR}$ or floxuridin ${ }^{\circledR}$ have yielded positive results only in the $7 \%$ and $16 \%$ of the cases, respectively. In a phase II study carried out with 3500 patients an objective response rate of $5.6 \%$ was obtained after using 72 anti-tumoral drugs [Yagoda1989]. And not much success has been achieved in this field during the last years [Yagoda1995].

These medicines are very toxic when administered at high doses, even if injected through a continuous infusion system adjusted to the circadian beat [Hrusesky1990, Dexeus1999]. Besides, a factor to be taken into account is the defensive mechanism of the renal cells carcinoma, which is based on the presence of glycoprotein P. This is a product of the gene MDR-1, and it is implied in the intracellular pumping mechanisms of toxic products to the outside of the cells, which to a certain extent could explain the resistance of the carcinoma renal cells to conventional chemotherapy [Mickish1994]. 
If a very high concentration of such chemotherapy agents could be concentrated inside a renal tumour, avoiding their systemic effects, eventually the defensive pumping mechanisms of the tumour cells could be overpassed, leading to tumour cell death. This is the starting hypothesis of the model, how to concentrate inside a tumour massive doses of a drug transported by a particulate system, leaving healthy tissue unaffected. We chose doxorubicin as the chemotherapy agent because it has been previously evaluated in combination with magnetic carriers [Wu2003, Jain2005]. The systemic administration of doxorubicin, like any other anti-tumour drug, has the serious inconvenience of its high toxicity. In its case it is manifested specially in the cardiac muscle.

Currently, medical treatment of renal tumours is mainly focused on the stimulation of the natural defences of the organism by the use of cytokines (interleukin and interferon) or the use of vaccines, with not too positive results. New drugs are also under development, mainly designed to block the growth factors of tumours. These drugs have achieved to slow down transitorily tumour growth, although in no case a real tumour regression has been observed [Tamaskar2008]. Therefore, it seems highly necessary to achieve a high impregnation of the tumour with the chemotherapy agents, avoiding as much as possible their systemic action.

Several strategies have been described to achieve an effective targeted accumulation of drug carriers into tumours, and only a very little number of them have been devoted to the theoretical or experimental development of magnetic implants. The classical approach has been to use a single source of magnetism to magnetize the carriers and attract them to a specific site inside the organism [Lübbe2001, Rudge2001, Flores2002]. This, however, presents many disadvantages, specially the impossibility to generate strong magnetic fields that permit a high capture efficiency of the carriers at the targeted site. 
To overcome this, Yellen et al. suggested with a theoretical approach the use of two independent magnetic sources. A small size magnetic mesh, consisting of a $3 \times 3$ array of magnetic wires, in combination with long range externally applied fields were used to magnetize and localize the carriers in an in-vitro experiment [Yellen2005]. They proved that fields in the range of 0.1Tesla should be used in order to reach magnetic saturation of both the particles and the implant. Fields over $0.1 \mathrm{~T}$ would have little additional effect on the particles capture. Therefore, their results would demonstrate that superparamagnetic nanoparticles at high concentrations can be captured at flow conditions consistent with the dimensions and flow velocity occurring in the coronary artery in the human blood.

Ritter and coworkers studied the feasibility of using a ferromagnetic wire implanted under the skin and next to the carotid artery for the collection of magnetic carriers using external magnets. A complete 2D mathematical analysis of the hydrodynamic and magnetic forces involved in the process was performed [Avilés2005]. Based on these calculations, they designed a coiled ferromagnetic wire stent in order to simulate an intravascular stent in-vitro. They used magnetite nanoparticles embedded in poly(styrene/divinylbenzene) matrixes as carriers, and an external permanent magnet to magnetize the wire stent. Changes at the capture efficiency by varying the applied magnetic field, the particle concentration and the fluid velocity were studied [Avilés2007]. They concluded that an increase in the fluid velocity and the concentration of particles had a positive effect on the efficiency of the capture of the magnetic carriers. This is due to the increase in the hydrodynamic force of the fluid and the formation of small aggregates. On the other hand, an increase in the applied magnetic field strength yielded a very slight improvement in the capture efficiency, since not very high fields were required for the saturation of particles and implants.

The implantation of small permanent magnets by minimally invasive surgery 
is a new alternative for magnetic targeting. Despite the preliminary achievements in in-vitro models, very little research has been performed in experimental animal models. This work reports some of the first in-vivo experiments of localisation of magnetic nanoparticles with implanted permanent magnets performed in animals [F.Pacheco2005, F.Pacheco2007].

As it was overseen in section 1.3.4, the effective delivery of magnetic carriers to the target has been usually approached by means of an external source of magnetism applied on the desired area to localise and concentrate magnetic particles. This method produces very low values for the intensity and the magnetic field gradient at the target region. The aim of the research carried out and described in this chapter is to validate a new approach, in which a permanent magnet allocated at the target organ is the source of magnetic field. The model is based on the implantation of small permanent magnets by minimally invasive surgery, which produces the localisation of magnetic particles at the desired zone.

Figure 6.1 shows the simulation of the magnetic field created by a Nd-Fe-B cylindrical permanent magnet. The dimensions of the magnet are $2 \mathrm{~mm}$ high and 4 $\mathrm{mm}$ diameter. The lines in the figure are the magnetic field lines, for the magnet magnetised along the vertical axis. The maximum number of field lines is found at the vicinity of the edge of the magnet, which means that maximum variation of field is achieved in this region (Fig. 6.2). Since magnetic field gradient, and not magnetic field intensity, is what really drives magnetic particles to move towards the magnet, maximum attraction of the particles shall be found near the edges of the magnet. These small, gold-coated permanent magnets generate a $3 \mathrm{KOe}$ field at the surface, being the magnetic field gradient approximately $30 \mathrm{~T} / \mathrm{m}$. 


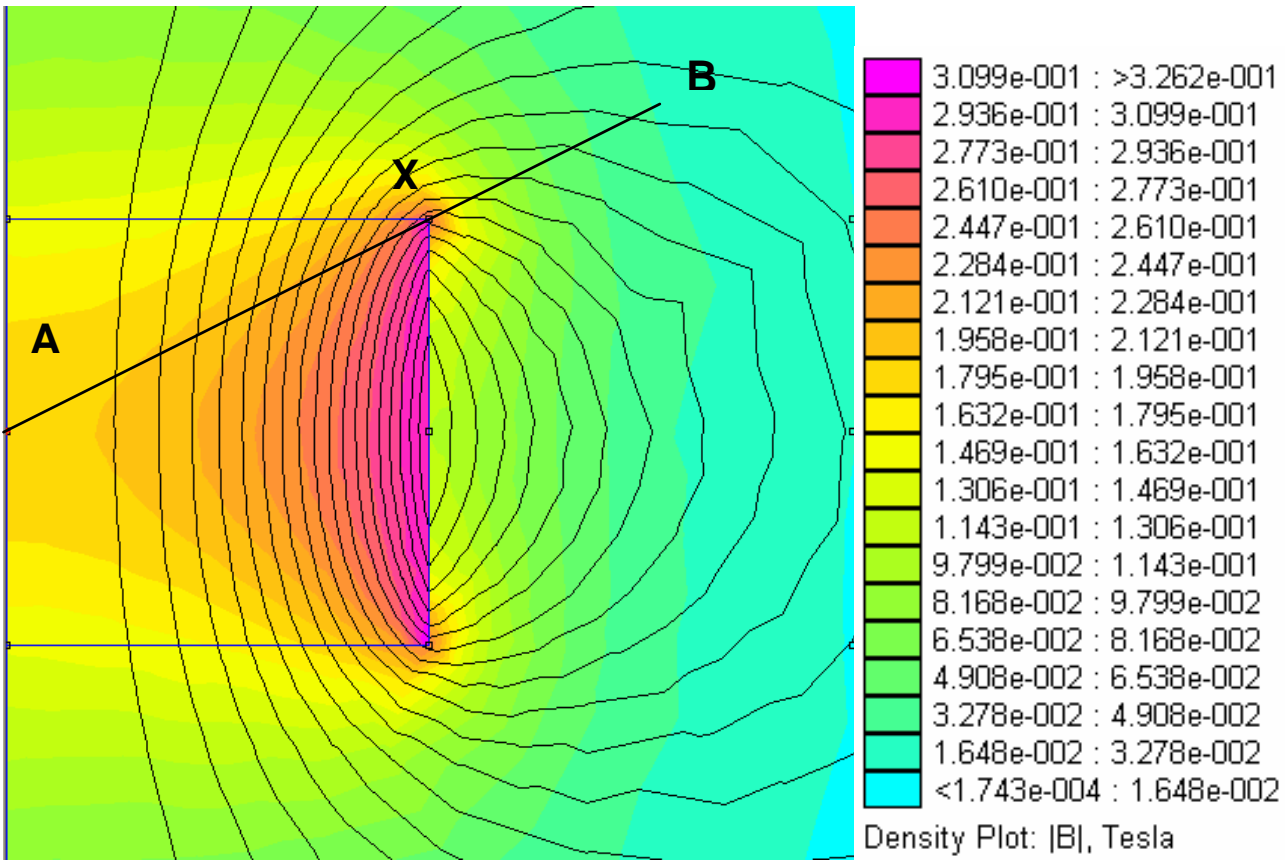

Figure 6.1 Simulation of the magnetic field created by a cylindrical permanent magnet. The figure represents half a magnet with cylindrical symmetry

Along with the use of a powerful and efficient source of magnetism that will retain the magnetic carriers at the tumour site, the second point is the choice of the adequate particles for drug delivery: Iron and iron oxide nanoparticles are generally well tolerated by the organism and can degrade with time [Ocón1994]. The ideal size for biomedical applications is below one micron, and preferred nanoparticles, or aggregates of particles size is below $200 \mathrm{~nm}$. Smaller particles have high surface areas and small cohesion forces, preventing that way aggregation before a magnetic field is applied. On the contrary, they have small magnetic susceptibility and saturation magnetisation, hence their magnetic response is much worse. A balance to meet both requirements is then necessary to achieve an efficient delivery of their drug load. 


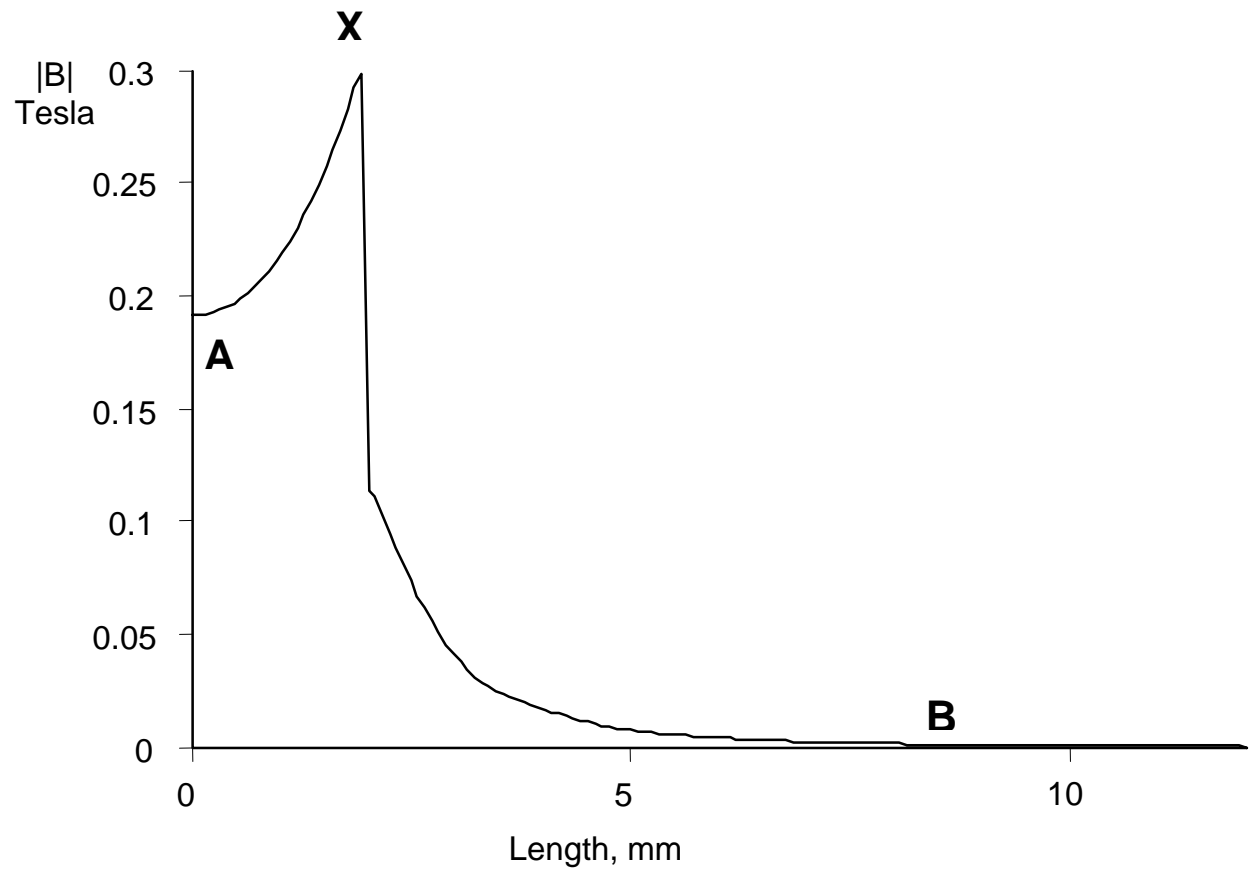

Figure 6.2 Variation of magnetic field with distance at the edge of the magnet.

This preclinical model is oriented to the local treatment of tumours with a difficult surgical approach, like meso-renal or hepatic multiple metastasis. Certain tumours, because of the difficulty of accessibility, cannot be directly extracted. The percutaneous implantation of a permanent magnet inside these tumours and the intravenous administration of drug magnetic carriers can increase the local chemotherapy effect, since the doses reached in-situ are very high. We also propose an alternative method to systemic delivery. It consists of an ecographic guided insertion of the magnetic carriers, which are retained near the zone of insertion of a permanent magnet to deliver their chemotherapy load over prolonged time. The clinical feasibility of the latter procedure has been checked experimentally, since the puncture with a 22 or 24 gauge needle produces less physical disturbances than the application of an insulin dose. 


\subsection{Results: overview}

All experiments were done at the Minimally Invasive Surgery Unit of the Veterinary Hospital of the University of Zaragoza (UTMI). The assistance of a very experienced team of co-workers was crucial to obtain the desired results. This unit is experienced in laparoscopy surgery and has been even performed percutaneous nefroscopy. Their experience has allowed achieving insertion of the magnet in one step by means of puncture and dilatation.

Experimental details are described in chapter 2. The use of a rabbit as the animal model presents many advantages, because of its size, easy handling and reproducibility. On the other hand, we observed that the overweight of the animals was an additional difficulty: in several cases fat covered completely the kidneys, impeding an easier approach to the target. Besides, the mobility of the organ also increases the experimental complications, since it has to be held in place by manual compression from the external part of the abdomen.

The magnet was successfully inserted in 52 rabbits (Fig. 6.3), in 4 of them the magnet was displaced out of the kidney, found outside the renal parenchyma or directly excreted via the urinary system, probably because the implantation had been done directly in the urinary tract. In 48 cases insertion was performed in the left kidney and in four of them it was done in the right one, as specified in the chirurgic protocol. In all the cases same chirurgic trauma was performed: a 12 french puncture in the opposite kidney so that both organs suffered an intraparenchyma hemorrhage, therefore serving as a control organ. In 54 cases laparoscopic technique was used. In other two animals medium supra- and infraumbilical laparatomy was carried out. In 41 animals the particles suspension was administered intravenously through the marginal ear vein. In seven cases the fluid was administered in a more central vessel, by means of a cutaneous mini-incision, 
three in primitive carotid artery and four in abdominal aorta artery. In 36 animals 2 $\mathrm{ml}$ of the $1 \%$ suspension in gelafundine were injected. In other three higher doses of commercially available nanoparticles were injected, and in another one $10 \mathrm{ml}$ of a 2\%Fe@C particles suspension.

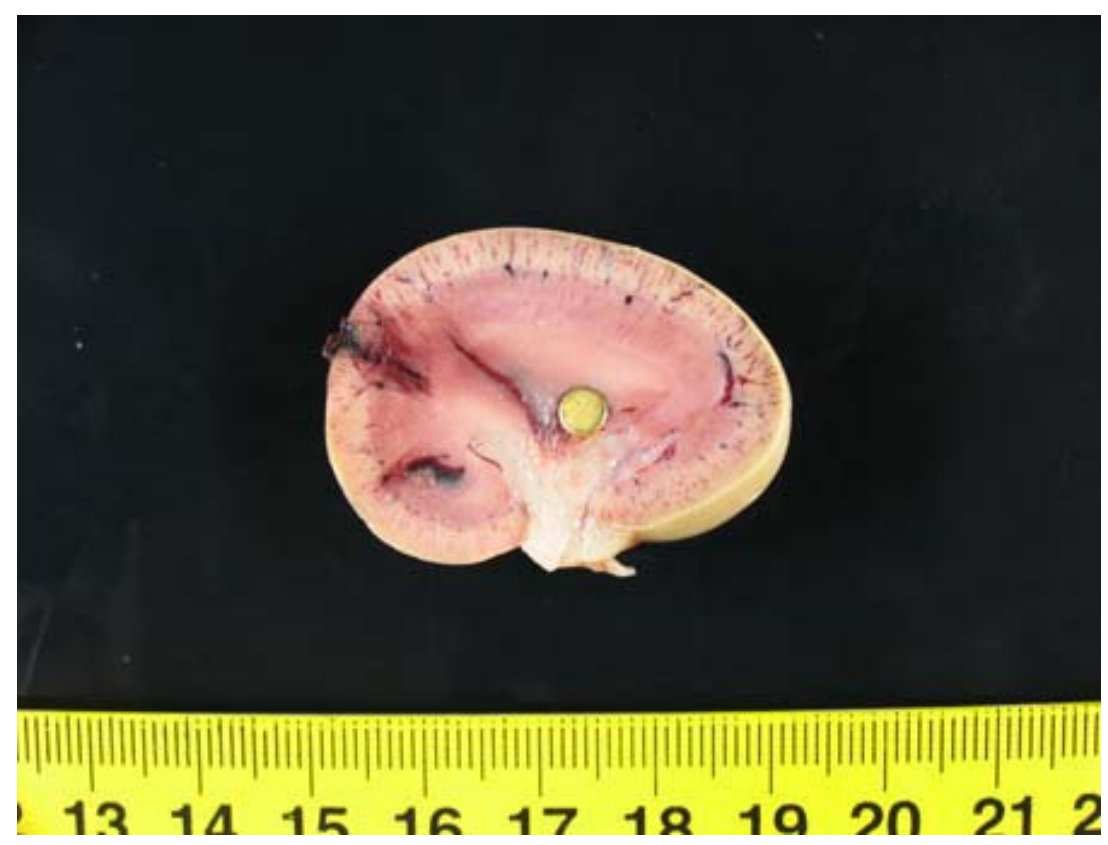

Figure 6.3 Longitudinal section of a rabbit's left kidney with a magnet inserted in the middle. Several haemorrhagic trajectories due to the insertion of the magnetic implant can be observed.

A transitory gas hyperpressure on the abdomen is enough to fulfil haemosthasia, that is, the stabilisation of the point of insertion without bleeding, avoiding the necessity of sutures. The approach was, in all cases, trans-peritoneal. Laparoscopy avoids the perforation of the intestines in an animal model, however in a human the approach could be retroperitoneal and guided by ultrasound scan, both trocarisation and insertion of the magnet.

In three cases intra-surgery death occurred after the insertion of the magnet. In 
two cases death was caused by anaesthesia failure, one because of kinking of the tracheal tube and another one due to accidental deflation of the balloon. In the other case death occurred after the injection of an overdose of particles. If $10 \mathrm{ml}$ of a $2 \%$ suspension was administered to a $4 \mathrm{Kg}$ rabbit, then the dose of $200 \mathrm{mg}$ is lethal. In other two cases death of the animals happened immediately after operation. In one of them immediate autopsy revealed the presence of haemorrhage coming from the kidney in which an implant had been set.

Thirty-five surviving animals were sacrificed after general terminal anaesthesia. 20 rabbits were sacrificed after $24 \mathrm{~h}$ form the insertion of the magnet. 25 animals were sacrificed after one week form the intervention. Two rabbits, operated by laparatomy, had to be sacrificed after 3 and 5 days because signs of an incipient evisceration were detected. Another animal died 24h after surgery because of mucous enteritis, not caused by the administration of the magnetic fluid. Histopathological study was carried out in all of the cases.

Initial results of the surgical experimental model are satisfactory, although the trauma produced during the insertion of the magnetic implants should be reduced. A 9 french spear is an alternative that allows the introduction of two cylindrical magnets of a smaller size: $3 \mathrm{~mm}$ diameter and $1.5 \mathrm{~mm}$ high. The introduction of these two magnets would create a similar magnetic field gradient to the one created by the single magnet previously used. A percutaneous procedure, exclusively directed by radioscopy, is under development. There is another possibility under study: the insertion of magnets tied to polypropylene wires, the extreme of which would be abandoned under the skin, in the cellular subcutaneous tissue. Once the treatment would be concluded, the magnet could be extracted by simple local anaesthesia. 
A good tolerance to the administration of particles was observed, since no cardiac stops or secondary effects, like partial or total limp of any of the animals was detected. Regarding the general state of survivor no significant changes in the vital parameters were objectivised.

In the moment of sacrifice, in the cases where minimally invasive surgery had been performed, a positive tolerance was detected, whereas eviscerations were found in some of the cases when laparotomy had been performed. During intervention no pus was detected. A slight peri-renal inflammation was detected in several cases, probably because surgery was not always performed in a completely aseptic environment. Non-magnetic material had to be used for the proper extraction of all of the organs to avoid undesired removal of the implant. Magnetic particles were found in many cases adhered to the surface of the magnet, proving its capacity to attract and retain magnetic material.

Histopathology examination proves, as expected, that the targeting of carbon coated iron nanoparticles injected intravenously inside the organism is hampered by the action of the immune system. Before reaching their target particles are filtered by the liver and spleen (Fig. 6.4). The size of big aggregates and the tendency of hydrophobic carbon to adsorb proteins in blood lead to their immediate recognition by kupffer cells, which remove them from circulation, impeding particles to reach their target in a considerable amount. Despite these problems the efficacy of the internal magnet can be checked in 25 out of 27 cases, when a significantly higher quantity of particles was found in the test organ compared to the control organ. Moreover, the presence of particles in the test organ was mainly due to the haemorrhage associated with the insertion of the magnet.

In the region of the kidney where particles were detected no macrophages were observed, only normal tissue, therefore the tolerance seems positive. This is 
in agreement with the results detected in-vitro, where no particles were detected inside macrophages in circulating blood. The capture of particles by the immune system is therefore restricted to the action of the filter organs.
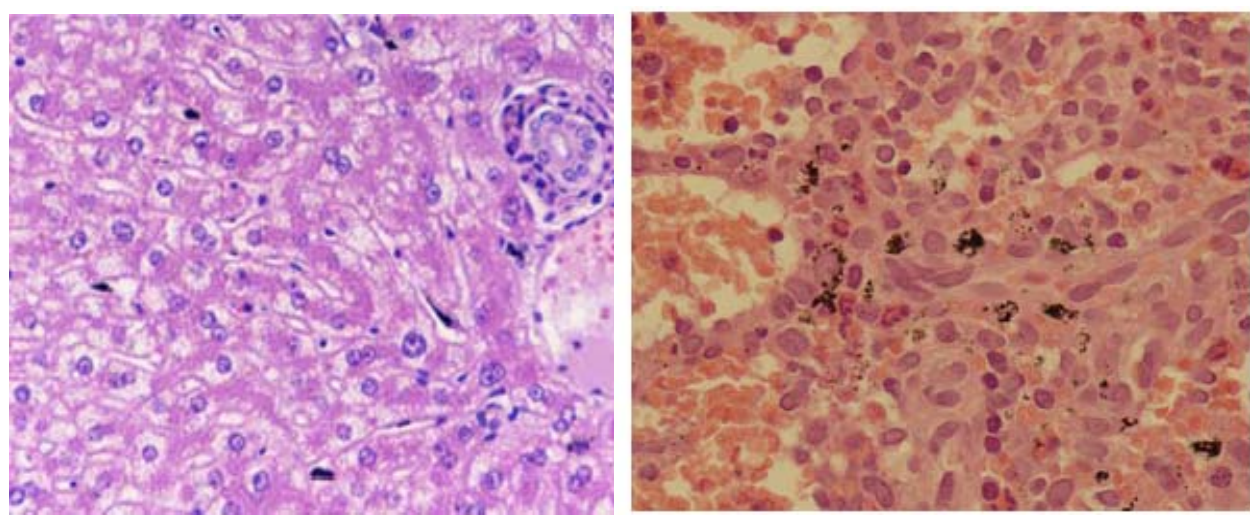

Figure 6.4 a) Liver capture of particles inside Kupffer cells. b) 5 Hyperaemic spleen with macrophages that contain nanoparticles and infiltration of neutrophiles. H.-E. X 400.

No studies of the local or general tolerance to doxorubicin have been performed, since the induction of tumours in living healthy tissue is a further step in this research. Once a higher concentration of particles at the desired target has been attained, even more adequate chemotherapy agents could be tested. Histopathology results are summarised in Table 6.1: Different groups of animals were sacrificed by terminal anesthesia after half an hour, 2, 7, 14, 21 and 28 days, and the main organs (lungs, liver, spleen, kidneys and bone marrow) were extracted for histological study. Table 6.2 is a summary of control animals. In order to assess the fate of the particles inside a living organism without the influence of a magnetic field, 8 rabbits were used as control animals. 2ml of a 1\% Fe@C particles in GFM were injected via the ear vein, and animals were sacrificed after 1, 7, 12 and 21 days after operation. Almost no particles were found inside the kidneys, whereas most of the particulate material was detected inside the lungs, liver and spleen. 
Table 6.1: Summary of Histopathology results. Legend: 1) Time of sacrifice 2) left kidney 3) right kidney 4) lungs 5) liver 6) spleen 7) Bone Marrow 8) Thymus 9) Type of administration 10) Incidences 11) Type of particle. *Insertion of magnet

\begin{tabular}{|c|c|c|c|c|c|c|c|c|c|c|c|}
\hline \# & 1 & 2 & 3 & 4 & 5 & 6 & 7 & 8 & 9 & 10 & 11 \\
\hline 1 & $30 \mathrm{~min}$ & $++*$ & + & ++++ & + & + & & & IV & & $\mathrm{FeC}$ \\
\hline 2 & $8 \mathrm{~h}$. & & + & + & + & - & & & IV & & $\mathrm{FeC}$ \\
\hline 3 & $30 \mathrm{~min}$ & $+*$ & + & & $++*$ & & & & IV & & $\mathrm{FeC}$ \\
\hline 4 & $30 \mathrm{~min}$ & $+++*$ & + & ++ & ++++ & +++ & & & IV & & $\mathrm{FeC}$ \\
\hline 5 & $1 \mathrm{~h}$. & $+++*$ & + & ++ & $++++*$ & ++++ & & & IV & & $\mathrm{FeC}$ \\
\hline 6 & $10 \mathrm{~min}$ & $++*$ & + & ++++ & ++++ & +++++ & & & IV & & $\mathrm{FeC}$ \\
\hline 7 & $30 \mathrm{~min}$. & $++*$ & + & ++ & ++++ & ++++ & & & IV & & $\mathrm{FeC}$ \\
\hline 8 & $30 \mathrm{~min}$. & $++*$ & + & + & +++ & +++ & & & IV & & $\mathrm{FeC}$ \\
\hline 9 & $30 \mathrm{~min}$. & $++*$ & + & + & +++ & +++ & & & IV & & $\mathrm{FeC}$ \\
\hline 10 & $36 \mathrm{~h}$ & $+*$ & - & - & +++ & +++++ & & & IV & & $\mathrm{FeC}$ \\
\hline 11 & $36 \mathrm{~h}$ & $++++*$ & - & + & ++++ & +++++ & & & IV & & $\mathrm{FeC}$ \\
\hline 12 & $36 \mathrm{~h}$ & $+*$ & - & + & ++++ & ++++ & & & IV & & $\mathrm{FeC}$ \\
\hline 13 & $36 \mathrm{~h}$ & ++ & $++++*$ & + & ++++ & ++++ & & & IV & & $\mathrm{FeC}$ \\
\hline 14 & $30 \mathrm{~min}$ & $+++*$ & +++ & +++ & +++++ & + & & & IV & & $\mathrm{FeC}$ \\
\hline 15 & $36 \mathrm{~h}$ & $+*$ & + & + & ++ & +++ & & & IV & & $\mathrm{FeC}$ \\
\hline 16 & $36 \mathrm{~h}$ & + & $++*$ & +++ & + & ++ & & & IV & 2 doses & $\mathrm{FeC}$ \\
\hline 17 & $36 \mathrm{~h}$ & $+*$ & ++ & ++++ & ++ & ++ & & & IV & 2 doses & $\mathrm{FeC}$ \\
\hline 18 & $36 \mathrm{~h}$ & $+*$ & - & ++++ & +++++ & +++++ & & & IV & & $\mathrm{FeC}$ \\
\hline 19 & $36 \mathrm{~h}$ & $+++*$ & + & +++++ & +++++ & +++++ & & & IV & & $\mathrm{FeC}$ \\
\hline 20 & $36 \mathrm{~h}$ & + & $++++*$ & +++++ & ++++ & ++ & & & IV & & $\mathrm{FeC}$ \\
\hline 21 & $36 \mathrm{~h}$ & $+++*$ & ++ & ++++ & + & ++++ & & & IV & & $\mathrm{FeC}$ \\
\hline 22 & $36 \mathrm{~h}$ & $++*$ & - & +++++ & ++ & + & & & IV & & $\mathrm{FeC}$ \\
\hline \multirow[t]{2}{*}{23} & $36 \mathrm{~h}$ & $++*$ & - & ++++ & ++ & ++ & & & IV & & $\mathrm{FeC}$ \\
\hline & & & & & & & & & IV & & \\
\hline 24 & $7 \mathrm{~d}$ & + & & + & + & + & & & IV & & $\begin{array}{l}\mathrm{Fe}- \\
\text { albumin }\end{array}$ \\
\hline 25 & $7 \mathrm{~d}$ & & & & & & & & IV & $\begin{array}{l}\text { No particles } \\
\text { were found }\end{array}$ & $\begin{array}{l}\mathrm{Fe}_{3} \mathrm{O}_{4^{-}} \\
\mathrm{PEG}\end{array}$ \\
\hline 26 & $7 \mathrm{~d}$ & $++*$ & & & & + & & & IV & & $350 \mathrm{~nm}$ \\
\hline
\end{tabular}




\begin{tabular}{|c|c|c|c|c|c|c|c|c|c|c|}
\hline 27 & $7 d$ & $++*$ & & + & + & + & & IV & & $80 \mathrm{~nm}$ \\
\hline 28 & $15^{\prime}$ & $+++*$ & + & + & & + & & $\begin{array}{l}\text { Aorta } \\
\text { Artery }\end{array}$ & & $\mathrm{FeC}$ \\
\hline 29 & $36 \mathrm{~h}$ & $++*$ & ++ & & & & & $\begin{array}{l}\text { Aorta } \\
\text { Artery }\end{array}$ & & $\mathrm{FeC}$ \\
\hline 30 & $36 \mathrm{~h}$ & $+++*$ & ++ & & + & ++ & & $\begin{array}{l}\text { Aorta } \\
\text { Artery }\end{array}$ & & $\mathrm{FeC}$ \\
\hline 31 & $15^{\prime}$ & $++*$ & & + & & & & $\begin{array}{l}\text { Aorta } \\
\text { Artery }\end{array}$ & & $\mathrm{FeC}$ \\
\hline 32 & $15 \mathrm{~d}$ & $\begin{array}{l}++++ \\
* \text { renal } \\
\text { capsule }\end{array}$ & & & & & & $\begin{array}{l}\text { Ultrasound } \\
\text { Scan guide }\end{array}$ & Clodronates & $\mathrm{FeC}$ \\
\hline 33 & $7 d$ & $+*$ & + & +++ & +++ & + & $\begin{array}{l}+ \\
+\end{array}$ & $\begin{array}{l}\text { Ultrasound } \\
\text { Scan guide }\end{array}$ & $\begin{array}{l}\text { Detachment } \\
\text { of gold }\end{array}$ & $\mathrm{FeC}$ \\
\hline 34 & $7 d$ & $+*$ & + & +++ & +++ & + & $\begin{array}{l}+ \\
+\end{array}$ & $\begin{array}{l}\text { Ultrasound } \\
\text { Scan guide }\end{array}$ & $\begin{array}{l}\text { Detachment } \\
\text { of gold }\end{array}$ & $\mathrm{FeC}$ \\
\hline 35 & $7 d$ & $*$ & & & + & + & & $\begin{array}{l}\text { Ultrasound } \\
\text { Scan guide }\end{array}$ & $\begin{array}{l}\text { Magnet } \\
\text { placed in } \\
\text { renal } \\
\text { capsule }\end{array}$ & $\mathrm{FeC}$ \\
\hline 36 & $7 d$ & $*$ & & & + & + & & $\begin{array}{l}\text { Ultrasound } \\
\text { Scan guide }\end{array}$ & $\begin{array}{l}\text { Detachment } \\
\text { of gold } \\
\text { coating }\end{array}$ & $\mathrm{FeC}$ \\
\hline 37 & $7 d$ & $+*$ & + & ++ & + & + & + & $\begin{array}{l}\text { Ultrasound } \\
\text { Scan guide }\end{array}$ & & $\mathrm{FeC}$ \\
\hline 38 & $7 d$ & $+*$ & + & + & + & + & & $\begin{array}{l}\text { Ultrasound } \\
\text { Scan guide }\end{array}$ & $\begin{array}{l}\text { Detachment } \\
\text { of gold } \\
\text { coating }\end{array}$ & $\mathrm{FeC}$ \\
\hline 39 & $30^{\prime}$ & $+++*$ & + & ++ & ++ & & + & $\begin{array}{l}\text { Carotid } \\
\text { Artery }\end{array}$ & & $\begin{array}{l}\mathrm{Fe}_{3} \mathrm{O}_{4^{-}} \\
\mathrm{SiO} 2\end{array}$ \\
\hline 40 & $30^{\prime}$ & $+++*$ & ++ & ++ & ++ & ++ & $\begin{array}{l}+ \\
+\end{array}$ & $\begin{array}{l}\text { Carotid } \\
\text { Artery }\end{array}$ & & $\mathrm{FeC}$ \\
\hline 41 & $9 \mathrm{~d}$ & $+*$ & +++ & & ++ & ++ & $\begin{array}{l}+ \\
+\end{array}$ & $\begin{array}{l}\text { Carotid } \\
\text { Artery }\end{array}$ & & $\begin{array}{l}\mathrm{Fe}_{3} \mathrm{O}_{4^{-}} \\
\mathrm{SiO}_{2}\end{array}$ \\
\hline
\end{tabular}




\begin{tabular}{|c|c|c|c|c|c|c|c|c|c|}
\hline 42 & $7 \mathrm{~d}$ & $+++*$ & & & & & $\begin{array}{l}\text { Ultrasound } \\
\text { Scan guide }\end{array}$ & & $\mathrm{FeC}$ \\
\hline 43 & $7 d$ & $+*$ & & & & & $\begin{array}{l}\text { Ultrasound } \\
\text { Scan guide }\end{array}$ & $\begin{array}{l}\text { Excretion of } \\
\text { magnet }\end{array}$ & $\mathrm{FeC}$ \\
\hline 44 & $7 d$ & $++*$ & & & + & ++ & IV & & $\begin{array}{l}\text { FeC- } \\
\text { PEG }\end{array}$ \\
\hline 45 & $7 d$ & $++*$ & & ++ & ++ & + & IV & $\begin{array}{l}\text { Detachment } \\
\text { of gold } \\
\text { coating }\end{array}$ & $\begin{array}{l}\text { FeC- } \\
\text { PEG }\end{array}$ \\
\hline 46 & $7 d$ & $*$ & & + & +++ & ++ & IV & & $\begin{array}{l}\text { FeC- } \\
\text { PEG }\end{array}$ \\
\hline 47 & $7 \mathrm{~d}$ & $+*$ & & + & + & + & IV & $\begin{array}{l}\text { Corrosion } \\
\text { of the } \\
\text { magnet }\end{array}$ & $\begin{array}{l}\text { FeC- } \\
\text { PEG }\end{array}$ \\
\hline 48 & $7 d$ & $*$ & & & +++ & ++ & IV & $\begin{array}{l}\text { Lungs edema } \\
\text { Displacement } \\
\text { of the magnet }\end{array}$ & $\begin{array}{l}\text { FeC- } \\
\text { RSA }\end{array}$ \\
\hline 49 & $7 \mathrm{~d}$ & $*$ & & & + & +++ & IV & $\begin{array}{l}\text { Corrosion } \\
\text { of the } \\
\text { magnet }\end{array}$ & $\begin{array}{l}\text { FeC- } \\
\text { RSA }\end{array}$ \\
\hline 50 & $7 \mathrm{~d}$ & $*$ & & + & + & + & IV & $\begin{array}{l}\text { Displaceme } \\
\text { nt of the } \\
\text { magnet }\end{array}$ & $\begin{array}{l}\text { FeC- } \\
\text { RSA }\end{array}$ \\
\hline 51 & $7 d$ & $*$ & & & ++ & + & IV & $\begin{array}{l}\text { Displaceme } \\
\text { nt of the } \\
\text { magnet }\end{array}$ & $\begin{array}{l}\text { FeC- } \\
\text { RSA }\end{array}$ \\
\hline 52 & $7 d$ & $+++*$ & & & + & + & IV & & $\begin{array}{l}\text { FeC- } \\
\text { RSA }\end{array}$ \\
\hline 53 & $7 \mathrm{~d}$ & $++*$ & & & & + & IV & $\begin{array}{l}\text { Detachment } \\
\text { of gold } \\
\text { coating }\end{array}$ & $\begin{array}{l}\text { FeC- } \\
\text { PEG }\end{array}$ \\
\hline 54 & $7 \mathrm{~d}$ & $++*$ & & + & + & + & IV & & $\begin{array}{l}\text { FeC- } \\
\text { PEG }\end{array}$ \\
\hline 55 & $\begin{array}{l}\text { Death } \\
24 \mathrm{~h}\end{array}$ & $++*$ & ++ & + & +++ & ++ & IV & & $\begin{array}{l}\text { FeC- } \\
\text { RSA }\end{array}$ \\
\hline 56 & $7 d$ & $+*$ & & & + & + & IV & & $\begin{array}{l}\text { FeC- } \\
\text { RSA }\end{array}$ \\
\hline
\end{tabular}


Table 6.2: Summary of histopathology in control animals. Legend: 1) Sacrifice 2) Left Kidney 3) Right kidney 4) Lungs 5) Liver 6) Spleen 7) Bone Marrow

\begin{tabular}{|c|c|c|c|c|c|c|c|}
\hline$\#$ & 1 & 2 & 3 & 4 & 5 & 6 & 7 \\
\hline 57 & $24 \mathrm{~h}$ & - & + & ++ & ++ & +++ & \\
\hline 58 & $24 \mathrm{~h}$ & + & + & - & - & - & \\
\hline 59 & $7 \mathrm{~d}$ & - & + & + & ++ & +++ & \\
\hline 60 & $7 \mathrm{~d}$ & - & - & + & +++ & +++ & \\
\hline 61 & $21 \mathrm{~d}$ & - & - & ++ & +++ & +++ & \\
\hline 62 & $21 \mathrm{~d}$ & - & - & + & ++ & + & \\
\hline 63 & $21 \mathrm{~d}$ & + & - & - & ++++ & ++++ & \\
\hline 64 & $12 \mathrm{~d}$ & - & - & ++ & ++++ & +++ & \\
\hline
\end{tabular}

\section{2 Intra-arterial administration}

For a better assessment of the efficacy of the selective magnetic interaction, and in order to avoid passing through the filters of the organism a larger amount of particles was injected through the carotid and abdominal aorta and renal arteries. That way particles could directly reach the target organs. In 6 of these cases there is an evident preference of distribution around the test control. A big quantity of particles was found localised in the zone of implantation, oriented towards the direction of the magnetic field gradient (figure 6.5). The injection of particles in artery is not an alternative to an ambulatory treatment that implies intravenous administration, since it requires minimum surgery and the access is much more difficult than in the case of veins. However, it is good proof of the efficacy of the method and the necessity to avoid the action of the immune system so as to effectively reach the target. 


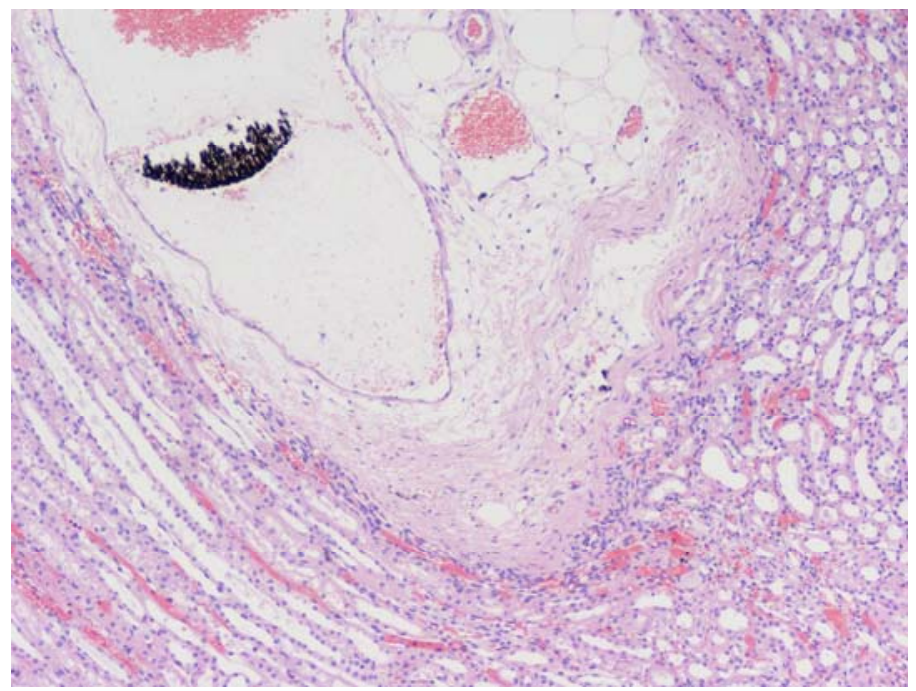

Figure 6.5 Particles accumulate in the vessel of a kidney in which a magnetic implant has been inserted. They remain there and tend to align in the direction of the magnetic field gradient.

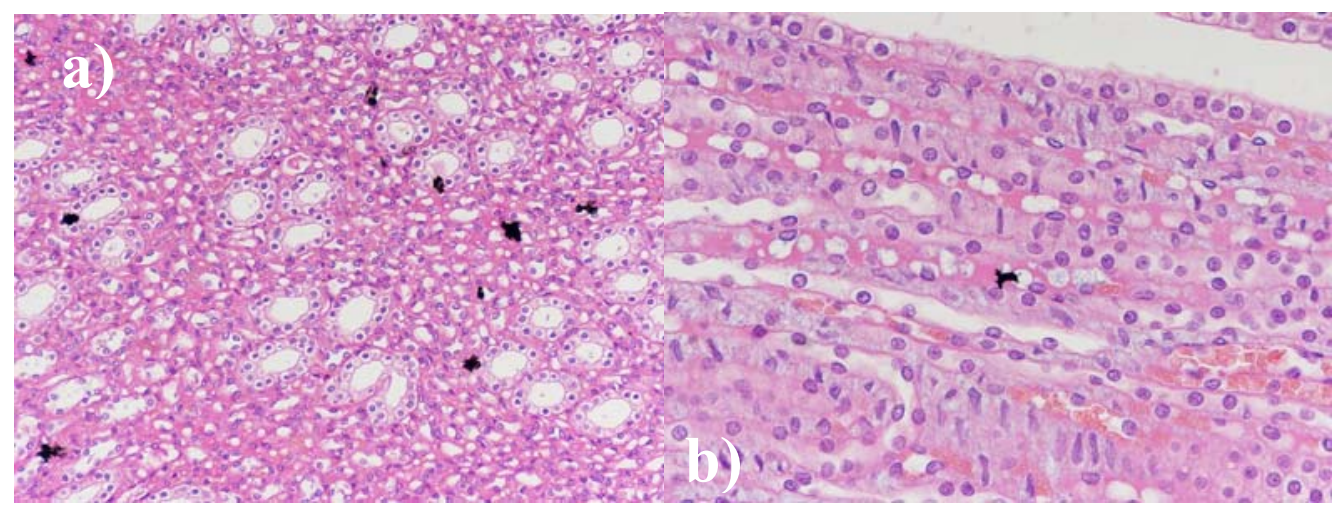

Figure 6.6 a) Cross-section of a left kidney, test organ. The presence of particles is evident inside the organ in which a magnet has been inserted. H.-E. X 200. b)Rigth kidney, control organ. Absence of nanoparticles on the non-implanted organ. H-E. X 200. 


\subsection{Ultrasound scan-assisted injection of the particles}

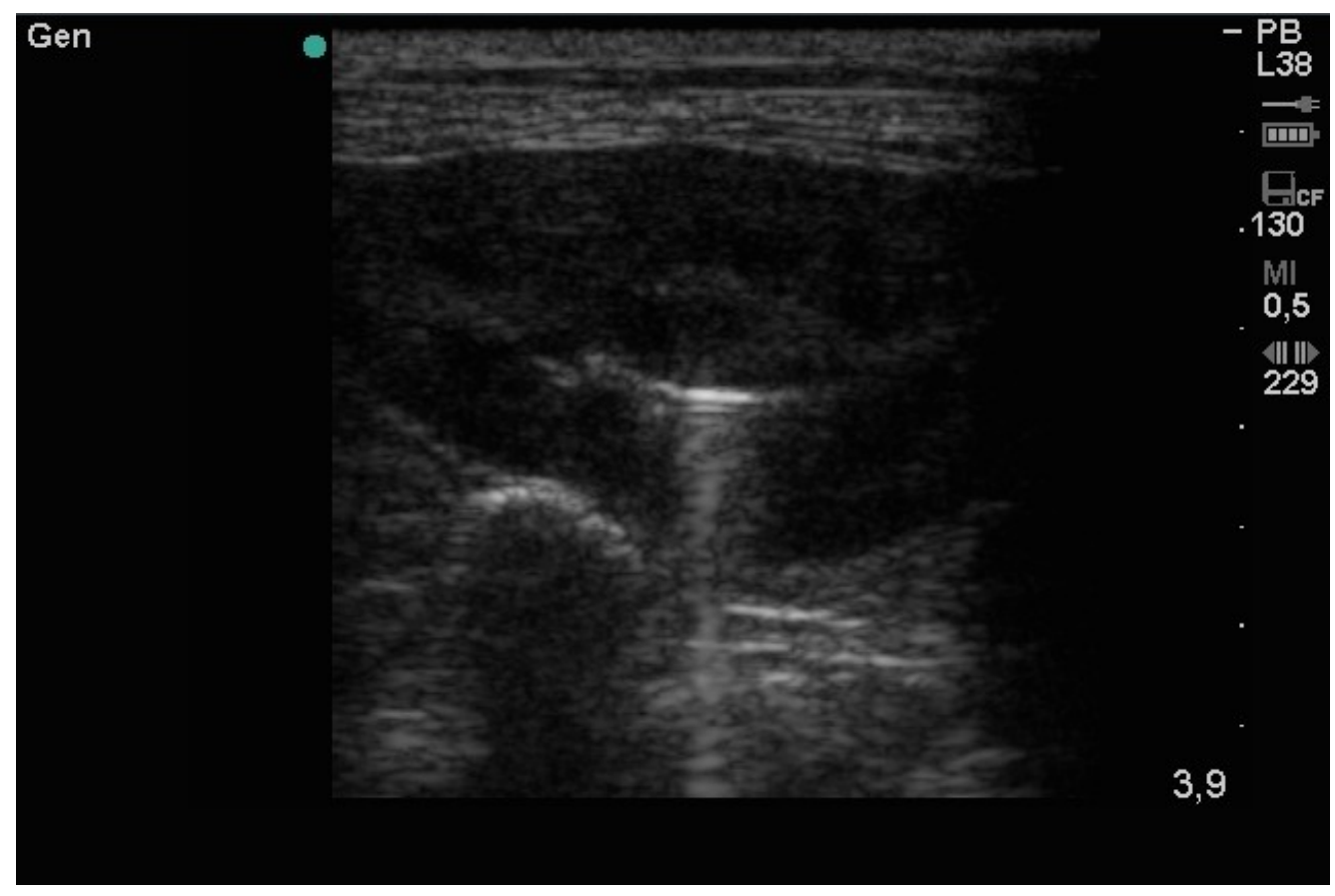

Figure 6.7: The ultrasound scan displays the image of a gold coated magnet inserted inside a kidney. After the injection magnetic nanoparticles remain attracted to the magnet, the amount of metallic then increases and the echo of the ultrasound is broadened

As an alternative to the intra-venous injection of the particles, a group of 9 animals underwent injection of the particles directly into the target organ with the aid of ultrasound scan equipment. This method allows visualising the position of the magnet and the direction of injection of the particles onto the desired zone. Seven days after the injection, $1 \mathrm{ml}$ of 1\% Fe@C particles in GFM were directly injected into the kidney carrying the magnetic implant. After the injection magnetic nanoparticles remain attracted to the magnet, the amount of metallic material increases. This is manifested by the broadening of the echo of the ultrasound. As expected, a very big quantity of particles was located inside the renal parenchyma 
(Fig. 6.8), even though a significant amount of magnetic material was still found inside the macrophages of liver and spleen.

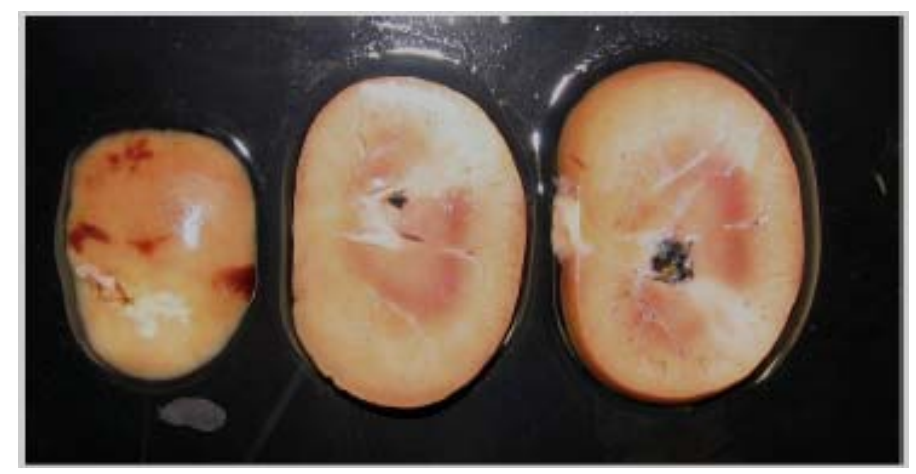

Figure 6.8 Macroscopic Image of the localistaion of the magnetic implant in the kidney. Nanoparticles, injected under ultrasound scan control, aggregate around the magnet.

The trajectory of injection of the particles is vital to reach the exact implant site. The use of a guide to direct the needle helps a better localisation of the particles around the zone influenced by the magnetic field. The perforation of the capsule of the kidney presents difficulties because of its fibrosity, whereas the renal parenchyma is softer. Besides, a kidney is a very mobile organ, and it slightly displaces during insertion, increasing the difficulty of operation. Once inserted, the exact identification of the magnet with the ultrasound scan is very easy. This facilitates enormously the direction of the needle for particle injection.

\subsection{Functionalised nanoparticles}

To avoid the action of the immune system and increase the average life time of particles in circulation, different encapsulations were tested. Commercial albumincoated particles and magnetite coated with PEG were injected, however the difficulty to detect these materials with the haematoxylin-eosin dye made it very complicated to evaluate their final destination inside the organism. Silica particles, 
injected in a great concentration via the carotid artery, were positively detected in one case, proving the selectivity of the particles towards the implanted organ.

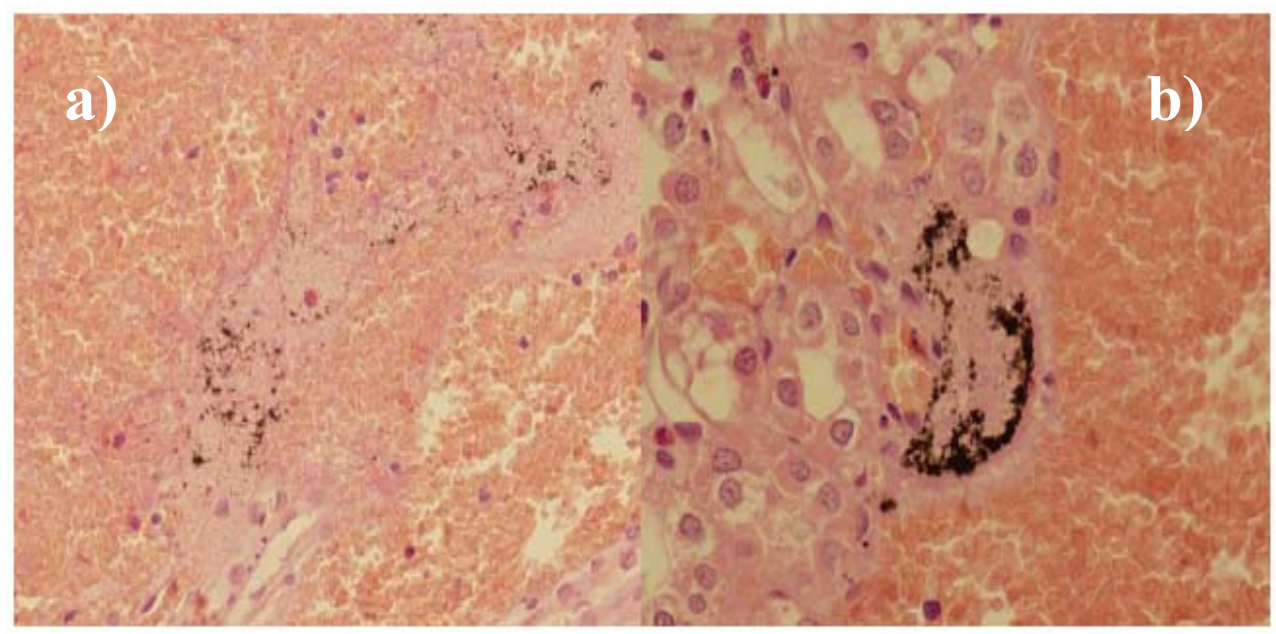

Figure 6.9 Left kidney, test organ. a) Nanoparticles in the traumatic haemorrhage zone caused by the implantation of the magnet. H.-E. X 200. b) Group of nanoparticles next to renal tubules and on the margins of the haemorrhage.

Particles functionalised with Rabbit serum albumin (RSA) and PEG were also injected intravenously, in 7 and 6 cases respectively. In 6 of them technical difficulties did not allow a correct study of the localisation of the particles, because of the corrosion of the core or displacement of the magnet. The presence of iron rust, positive to Blue Pearls dye, is a symptom of the corrosion of the coating which encapsulates the sintered Nd-Fe-B magnet. In one animal the magnetic implant had migrated to the ureter, identified by X-Rays. Regarding the RSA coating, results are not reproducible or especially conclusive. Only in one case the amount of particles found on the test organ was significant compared to the control organ, and in any case the apparition of particles inside the macrophages in a large extent means that the serum albumin coating has not produced the desired stealth effect. In two animals strong lungs infection were detected after the administration 
of the Fe@C - RSA fluid. Even though there could be reactions of refusal of the organism to a foreign protein, like commercial RSA, these infections seem to be due only to diseases of the animals before treatment.

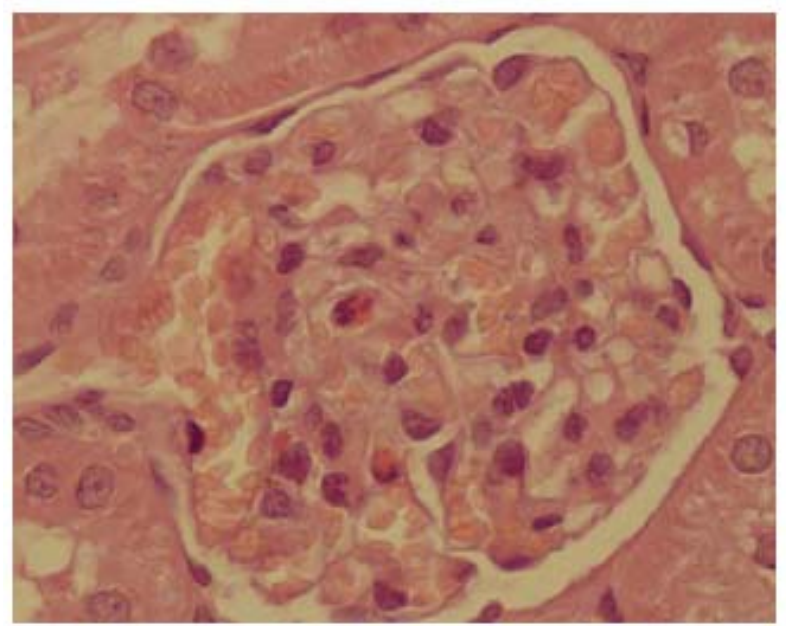

Figure 6.10 Detail of a hyperaemic glomerulus and the presence of plinuclear neutrophiles. H.-E. X 400.

In four cases in which Fe@C - PEG particles were injected, a significant concentration of particles was found in the test organ, localised in the zone of implantation or generalised inside the renal parenchyma (Fig 6.9). And, more importantly, the coating of the particles really seemed to have diminished the amount of particles captured by the macrophages of spleen, and especially kupffer cells inside the liver. The hydrophilic PEG coating, and the improvement in the stabilisation of the particles in aqueous suspension after the incorporation of the polymeric coating increases the permanence of particles in circulation in blood, thus increasing the probability to reach the target organ without being removed by the immune system. These are anyhow preliminary results, and the process of coating the particles with more adequate PEGs is still to be optimised. 


\subsection{Summary of results}

The experimental model proves that the laparoscopic insertion of a small magnetic implant inside the renal parenchyma is an unprecedented, effective and easily reproducible method to deliver magnetic carriers to a target organ. The tolerance of well-coated, biocompatible magnets is excellent, and its position can be easily tracked after operation by means of abdominal ultrasound scan or X-Ray. During the development of the surgical model the anaesthesia procedure was improved, being the endotracheal intubation eventually unnecessary because of the reduction in surgery time. The elimination of the endotracheal intubation led to a reduction in morbility associated to surgery.

Regarding surgical equipment, the dimensions of the used trocar are too big for the size of a rabbit's kidney. Same chirurgic procedure in humans is expected to cause lesser trauma. The approach for the insertion of the magnets is $1 \mathrm{~cm}$, a tolerable length in a human organism. In human urological surgery (percutaneous nephrostomy or percutaneous nephroscopy) it is possible to reach in the kidney parenchyma tract a diameter of $1 \mathrm{~cm}$ without haemorrhagic difficulties, but in rabbit the diameter of the used trocar is, in proportion, much bigger.

An alternative to these experiments could be the insertion of magnets of a smaller diameter through narrower trocars, in order to avoid possible traumas caused by the surgery procedure. Other possibilities envisage the utilisation of magnets inside a capsule of titanium, or with a sharpened edge. A prospect in the future is the use of polypropylene wires attached to the magnet, coiled up and fixed subcutaneously for an easier removal of the magnet and the magnetic material after treatment has been completed. 


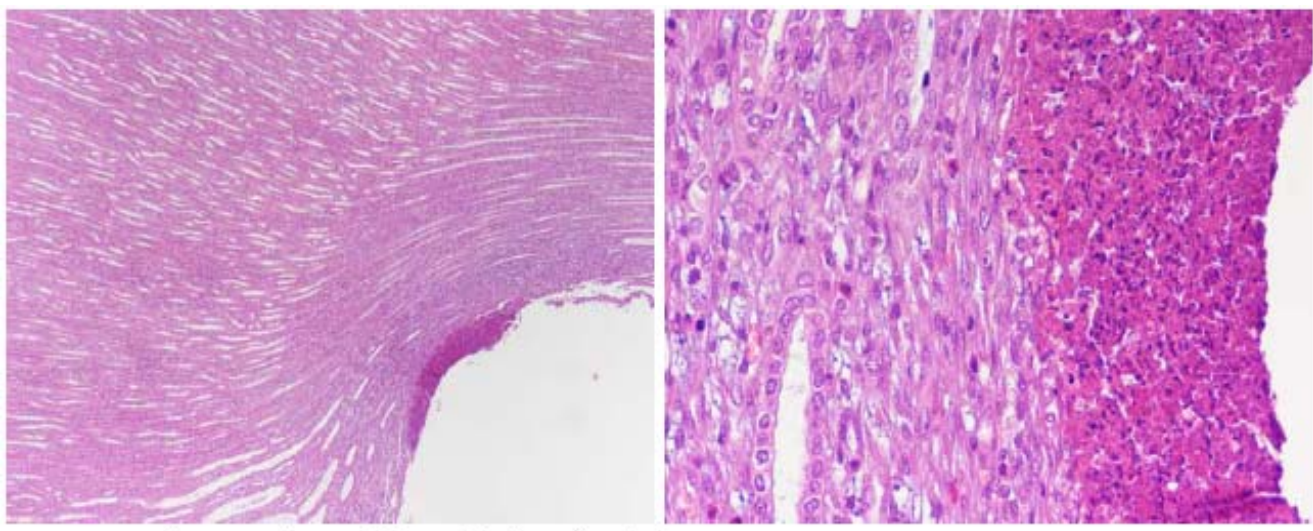

Figure 6.11 Residual cavity left after the extraction of the magnet (right down corner). H.E. X 40. b) In detail, necrotic material on the margin of the cavity left by the removal of the magnet.

Histopathology studies show the distribution inside the organism and the short-term and medium-term tolerance of nanoparticles inside the main organs of the body. Particles are attracted under the influence of the internally created magnetic field gradient, and are retained near the magnet. In the case of drugloaded particles this will permit a slow and controlled diffusion of the chemotherapy agent in the desired zone.

All the results obtained in histological analysis performed in samples obtained from different organs, showed the lack of Perls stain reaction, as was also observed in haematological test. These results confirmed the good encapsulation of the magnetic particles core, as confirmed by the morphological characterisation of the particles. From histological studies, we observed a larger particles concentration in the implanted kidney in comparison to the non-implanted one. Although it is difficult to quantify the results, a significant quantity of the particles concentrates on the kidney in which a magnet has been inserted. This is an indication of the capability of the magnet to attract particles specifically to the target organ. This is 
the key issue to the method, and the main advantage of using internal magnets over the use of external magnetic fields. A relatively important amount of the particles are found to concentrate on the main filters of the body: liver and spleen, whereas a quite lower quantity of particles have been found in the bone marrow (Fig. 6.12) and lungs of some animals (Fig. 6.13).

Despite the inert surface of our material, the size of the particles is big enough to be recognised by the macrophages of these organs, which capture them and retain them, eventually excreting them after several days. This fact could be a drawback for the purpose of delivering chemicals to specific targets in the body but, on the other hand, it could also be helpful for other applications, such as treatment of liver or spleen tumours, or for the treatment of leishmania. [Chellat2005]. The obtained results have opened a new research direction that is being developed to confirm these assertions.

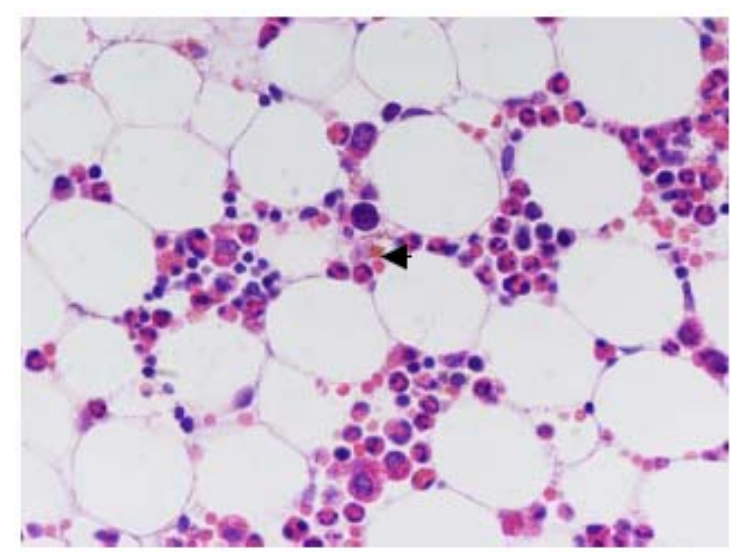

Figure 6.12. Bone marrow. A very low amount of particles was found, pointed with an arrow on the image. H.-E. X 600. 


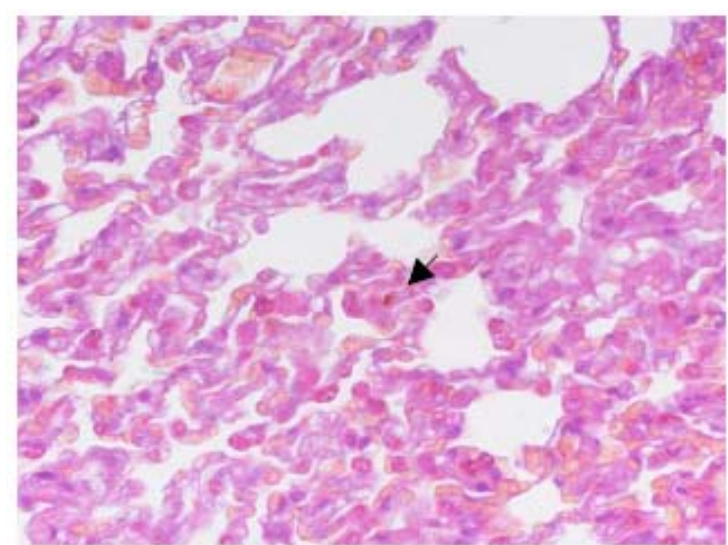

Figure 6.13. A very low concentration of particles was found in lungs. H.-E. X 600.

The injection of particles directly inside the abdominal aorta artery is a way to avoid the withdrawing of the particles by the Reticuloendothelial System. That way we have confirmed the ability of the magnet to actually concentrate the particles in the target organ. More work needs to be done in order to avoid the action of macrophages of the liver and spleen. These organs retain some of the particles, preventing them from reaching the desired goal. The functionalisation of the nanoparticles' surface with chemical groups that specifically avoid opsonisation and repel the macrophages is a profitable solution. This led to the necessity of a hydrophilic coating with poly (ethyleneglycol). Given the fact that the internal magnet actually concentrates a big amount the magnetic material, the use of smaller particles, under the limit of detection of the body $(100 \mathrm{~nm})$ is another interesting possibility. 



\section{Summary and conclusions}

The work carried out throughout this doctoral thesis concerns the synthesis, characterisation and functionalisation of magnetic nanoparticles for biomedical applications. With this aim, four novel types of particles have been synthesised: iron encapsulated in carbon $(\mathrm{Fe} @ \mathrm{C})$ and iron encapsulated in silica $\left(\mathrm{Fe} @ \mathrm{SiO}_{2}\right)$, by the arc-discharge method; megnetite embedded in zeolite $\mathrm{Y}\left(\mathrm{Fe}_{3} \mathrm{O}_{4} @ \mathrm{ZY}\right)$ by mechanical milling; and magnetite crystallites encapsulated in amorphous silica $\left(\mathrm{Fe}_{3} \mathrm{O}_{4} @ \mathrm{SiO}_{2}\right)$ by a combination of the co-precipitation method and the sol-gel technique. All four types of particles have been characterised morphologically, by electron microscopy tecniques, magnetically at the SQUID magnetometer and chemically, by IR and VIS-UV spectroscopy, and by $\mathrm{N}_{2}$ adsorption following the BET method.

The discharge-arc method is a non expensive, simple way to obtain perfectly encapsulated, highly magnetic particles. Fe@C and $\mathrm{Fe} @ \mathrm{SiO}_{2}$ are ferromagnetic and present aggregates of particles of several hundreds of nanometers in suspension. $\mathrm{Fe} @ \mathrm{SiO}_{2}$ presented the highest saturation magnetisation referenced in bibliography. $\mathrm{Fe}_{3} \mathrm{O}_{4} @ \mathrm{ZY}$ preserves the high surface area and the crystallinity of the starting zeolite after the mechanical activation with magnetite. $\mathrm{Fe}_{3} \mathrm{O}_{4} @ \mathrm{SiO}_{2}$ are superparamagnetic crystallites with a high surface area. TEM proved the perfect encapsulation of all magnetic cores. Stable aqueous suspensions of all particles have been obtained in order to use them in in-vitro and in-vivo applications in Biomedicine.

Biocompatibility of the obtained nanoparticles has been analised in-vitro. Studies of Coagulation, platelet aggregation, variations in blood and plasma viscosity and a complete rheological study, both in human and in rabbit circulating 
blood have been performed. In all cases the results obtained were within tolerable limits. The capability of particles to be uptaken by living cells has also been studied. Dendritic cells are able to internalise Fe@C particles, as proved by electron microscopy and magnetic measurements.

$\mathrm{Fe} @ \mathrm{C}$ and $\mathrm{Fe} @ \mathrm{SiO}_{2}$ particles have been functionalised with carboxyl and amine groups on their surface to bind to the specific monoclonal antibody directed against the human chorionic gonadotropin hormone (hCG). The presence of the antibody was detected by XPS measurements and by detection with a lateral-flow immunoassay. This way, functionalised particles could be used for a more effective delivery to a target zone: the grafting of a specific antibody to the surface of a magnetic particle allows magnetic targeting and molecular recognition at the same time.

In order to delay the action of the immune system inside the organism, and therefore allow a circulation of the particles for a longer time in blood, $\mathrm{Fe} @ \mathrm{C}$ particles have been coated with the copolymer PEG and the serum albumin protein. The effectiveness of the coating was assessed by XPS and VIS-UV spectroscopy, respectively.

The adsorption and release of the chemotherapy agent doxorubicin was studied for $\mathrm{Fe} @ \mathrm{C}, \mathrm{Fe}_{3} \mathrm{O}_{4} @ \mathrm{ZY}$ and $\mathrm{Fe}_{3} \mathrm{O}_{4} @ \mathrm{SiO}_{2}$ particles. Al three materials show an appreciable loading capacity of adsorption and release of the chemotherapy agent doxorubicin. Adsorption is fast and achieved within a few hours, whereas complete release takes place after several days. This controlled release would be slow enough to allow the carriers to reach the target and desorb the load only at the specific site. $\mathrm{Fe}_{3} \mathrm{O}_{4} @ \mathrm{ZY}$ has the maximum loading capacity but a faster release, related to a superficial adsorption of the drug on the surface of the particles.

The laparoscopic insertion of a small magnetic implant inside a solid organ is 
an alternative to conventional chemotherapy and externally-driven magnetic drug delivery. A preclinical experimental model is described. Kidneys were chosen because of their symmetry and good vascularisation, and the method proved to be effective for the concentration of magnetic nanoparticles at the zone of implantation. The direct injection of particles inside the target organ via a long needle is another possibility. It can be easily tracked by means of abdominal ultrasound scan.

Histopathology studies showed a bigger concentration of particles in the implanted kidney in comparison to the non-implanted one. Although it is difficult to quantify the results, a significant quantity of the particles concentrates on the kidney in which a magnet has been inserted. This is an indication of the capability of the magnet to attract particles specifically to the target organ.

The injection of particles directly inside the abdominal aorta artery also confirmed the ability of the magnet to actually concentrate the particles in the target organ. Some of the particles are retained in the main filters of the body: lungs, liver and spleen. Pegylation of particles avoids opsonisation, allowing a longer time of circulation in bloodm leading to a bigger concentration of particles in the target organ, and a lesser amount of magnetic material in liver and spleen. 



\section{List of abbreviations}

APC: Antigen presenting cell

APTES: Aminopropyl triethoxysilane

BET: Brunauer-Emmet-Teller method for the determination of surface areas

BFTEM: Bright Field Transmission Electron Microscopy

BSA: Bovine Serum Albumin

DC: Dendritic Cell

DFTEM: Dark Field Transmission Electron Microscopy

DLS: Dynamic Light Scattering

EFTEM: Energy Filtered Transmission Electron Microscopy

FFT: Fast Fourier Transform

FTIR: Fourier-transform Infra Red Spectroscopy

FWHM: Full-width-half-maximum

GFM: Gelafundine

HEBM: High Energy Ball Milling

HRTEM: High Resolution Transmission Electron Microscopy 
IR: Infrared Spectroscopy

MDC: Dendritic cell obtained from myelomonocytic progenitors

MIH: Magnetic Inductive Hyperthermia

MNP: Magnetic Nanoparticle

MRI: Magnetic Resonance Imaging

NMR: Nuclear Magnetic Resonance

NPs: Nanoparticles

PBMA: Poly (butylmethacrylate)

PBS: Phosphate Buffer Solution.

PCS: Photon Correlation Spectroscopy

PEG: Poly (ethyleneglycol)

PGMA: Poly (glycidylmethacrylate)

PMAO: Poly (maleic anhydride aminooctadecene)

PRB: Particles Resuspension Buffer

PVA: Poly Vinylalcohol

RES: Rethiculoendothelial system 
RSA: Rabbit Serum Albumin.

SAED: Selected Area Electron Diffraction

SBA-15: Silica mesoporous sieve with hexagonal channels

SDS: Sodium Dodecyl Sulphate

SEM: Scan Electron Microscopy

SLP: Specific Loss Power

SPION: Superparamagnetic Iron Oxide Nanoparticle

SQUID magnetometer: Superconducting Quantum Interference Device

TEOS: Tetraorthosilicate

TEM: Transmission Electron Microscopy

USPION: Ultra-small Superparamagnetic Iron Oxide Nanoparticle

UV-VIS: Ultraviolet-Visible Spectroscopy

VEGF: Vascular Endothelial Growth Factor

XPS: X-Ray Photoelectron Spectroscopy

XRD: X-Ray Diffraction

ZY: Y Zeolite 



\section{Bibliography}

Ahrens, E. T.; Feili-Hariri, M.; Xu, H.; Genove, G.; Morel, P. A. Magn .Reson. Med. 49 (2003) 1006.

Alexiou, C.; Arnold, W.; Klein, R. J.; Parak, F.; Hulin, P.; Bergemann, C.; Erhardt, W.; Wagenpfel, S.; Lübbe, A. Cancer Res. 60 (2000) 6641.

Alexiou, C.; Jurgons, R.; Schmid, R.; Hilpert, A.; Bergemann, C.; Parak, F.; Iro, H. J. Mag. Mag. Mat., 293, (2005) 389.

Ambe, K.; Mori, M.; Enjoji, M. F. Am. J. Surg. Pathol. 11 (1987) 310.

Anderson, S. A. Glod, J.; Arbab, A. S.; Noel, M.; Ashari, P.; Fine, H. A.; Frank, J. A. Blood 105 (2005) 420.

Arroyo-Hernández, M.; Martín-Palma, R. J.; Pérez-Rigueiro, J.; García-Ruiz, J. P.; García-Fierro, J. L.; Martínez-Duart, J. M. Mater. Sci. Eng. C 23 (2003) 697.

Arruebo, M.; Fernández-Pacheco, R.; Ibarra, M. R.; Santamaría, J. Nanotoday 2 (2007) 22 (a).

Arruebo, M.; Fernández-Pacheco, R.; Velasco, B.; Marquina, C.; Arbiol, J.; Irusta, S.; Ibarra, M. R.; Santamaría, J. Adv. Funct. Mater. 2 (2007) 2 (b).

Arruebo, M.; Galán, M.; Navascués, N.; Téllez, C.; Marquina, C.; Ibarra, M. R.; Santamaría, J. Chem. Mater; 18 (2006) 1911 (a). 
Arruebo, M.; Fernández-Pacheco, R.; Irusta, S.; Arbiol, J.; Ibarra, M. R.; Santamaría, J. Nanotechnology 17 (2006) 4057 (b).

Artemov, D.; Mori, N.; Okollie, B.; Bhujwalla, Z. M. Magn. Reson. Med. 49 (2003) 403.

Astorino, E.; Peri, J.; Willey, R.; Busca, G. J. Catal. 157 (1995) 482.

Atici, O. G.; Akar, A.; Rahimian, R. Turk. J. Chem. 25 (2001) 259.

Avilés, M. O.; Chen, H.; Ebner, A. D.; Rosengart, A. J.; Kaminski, M. D.; Ritter, J. A. J. Magn. Magn. Mater. 311 (2007) 306.

Avilés, M. O.; Ebner, A. D.; Chen, H.; Rosengart, A. J.; Kaminski, M. D.; Ritter, J. A. J. Magn. Magn. Mater. 293 (2005) 605.

Banchereau, J.; Steinman, R. M. Nature 392 (1998) 245.

Banerjee, I.; Khollam, Y.; Balasubrmanian, C.; Pasricha, R.; Bakare, P.; Patil, K.; Das, A.; Bhoraskar, S. Scripta Mater. 54 (2006) 1235.

Bangs Laboratories, Inc. Covalent coupling (www.bangslabs.com). TechNote 205. Rev. 003 (2002) Page 5.

Bao, H.; Chen, Z.; Kang, L.; Wu, P.; Liu, J. Mater. Lett. 60 (2006) 2167.

Barfield, R. C.; Otto, M.; Houston, J.; Holladay, M.; Geiger, T.; Martin, J.; Leimig, T.; Gordon, P.; Chen, X. Cytotherapy 6 (2003) 1.

Bautista, M. C.; Bomatí-Miguel, O.; Morales, M.P.; Serna, C. J.;VeintemillasVerdaguer, S. J. Magn. Magn. Mater. 293 (2005) 20. 
Bomatí-Miguel, O.; Leconte, Y.; Morales, M.P.; Herlin-Boime, N.; VeintemillasVerdaguer, S. J. Magn. Magn. Mater. 290 (2005) 272.

Bomatí-Miguel, O.; Morales, M. P.; Tartaj, P.; Ruiz-Cabello, J.; Boneville, P.; Santos, M.; Zhao, X.; Veintemillas-Verdaguer, S.; Biomaterials 26 (2005) 5695.

Bonnemain, B., J. Drug Target. 6 (1998) 167-74

Brigger, I.; Dubernet, C.; Couvreur, P. Adv. Drug Deliv. Rev. 54 (2002) 631.

Bruce, I. J.; Taylor, J.; Todd, M.; Davies, M. J.; Borioni, E.; Sangregorio, C.; Sen, T. J. Magn. Magn. Mater. 284 (2004) 145.

Brunauer, S., Emmett, P. H., Teller, E., J. Am. Chem. Soc. 60 (1938) 309.

Brydson, Rik. Electron energy loss spectroscopy. Garland/BIOS Scientific Publishers (2006).

Bulte, J. W. M.; Kraitchman, D. L. NMR Biomed. 17 (2004) 484.

Burt, R.; Pearce, W.; Luo, K.; Oyama, Y.; Davidson, C.; Beohar, N.; Gheorghiade, M. Bone Marrow Transplant 32 (2003) 29.

Cao, G. Nanostructures and Nanomaterials: Synthesis, properties and applications. Imperial College Press, London, 2004.

Cao, J.; Wang, Y.; Yu, J.; Xia, J.; Zhang, C.; Yin, D.; Häfeli, U.O. J. Magn. Magn. Mater. 277 (2004) 165.

Carmeliet, P.; Jain, R. K. Nature 407 (2000) 249.

Chellat, F.; Merhi, Y.; Moreau, A.; Yahia, L. Biomaterials 26 (2005) 7260. 
Chen, H.; Kaminski, M. D.; Liu, X.; Mertz, C. J.; Xie, Y.; Torno, M.; Rosengart, A. J. Med. Hypotheses 68 (2007) 1071.

Cheng, J.; Ni, X.; Zheng, H.; Li, B.; Zhang, X.; Zhang, D. Mater. Res. Bull. 41 (2006) 1424 (a).

Cheng, J. J., Teply, B.A., Jeong S.Y. Pharm. Res. 23 (2006) 557 (b).

Christine-Lutsiak, M. E.; Robinson, D. R.; Coester, C.; Kwon, G.S.; Samuel, J. Pharm. Res. 19 (2002) 1480.

Coester, C.; Nayyar P.; Samuel, J. Eur. J. Pharm. Biopharm. 62 (2006) 306.

Colliex, C.; Manoubi, T.; Ortiz, C. Phys. Rev. B 44 (1991) 11402.

Conejo-Garcia, J. R.; Benencia, F.; Courreges, M. C.; Kang, E.; Mohamed-Hadley, A.; Buckanovich, R. J.; Holtz, D. O.; Jenkins, A.; Na, H.; Zhang, L.; Wagner, D. S.; Katsaros, D.; Caroll, R.; Coukos, G. Nature Med. 10 (2004) 950.

Conejo-Garcia, J. R.; Buckanovich, R. J.; Benencia, F.; Courreges, M. C.; Rubin, S. C.; Carroll, R. G.; Coukos, G. Blood 105 (2005) 679.

Coppola, D.; Fu, L.; Cicosia, S. V.; Kounelis, S.; Jones, M. Hum. Pathol. 29 (1998) 455 .

Costa, P.; Ferreira, D. C.; Sousa Lobo, J. M. Rev. Port. Farm. 46 (1996) 4.

Costa, P.; Sousa Lobo, J. M. Eur. J. Pharm. Sci. 13 (2001) 123.

Crangle, J.; Goodman, G. M. Proc. R. Soc. London, Ser. A, Mat. Phys. Sci. 321 (1971) 477. 
De Teresa, J. M.; Marquina, C. I.; Serrate, D.; Fernández-Pacheco, R.; Morellón, L.; Algarabel, P. Ibarra, M. R. Int. J. Nanotech. 2 (2005) 3.

De Teresa , J. M.; Velasco, B.; Grazú, V.; Fernández-Pacheco, R.; Marquina, C. I.; Sauriel, D.; Valero, J. A.; Ibarra, M. R. Submitted to Bioelectronics and Biosensors (2008).

De Vries, I. J. M.; Lesterhuis, W. J.; Barentsz, J. O.; Verdijk, P.; Van Krieken, J. H.; Boerman, O. C.; Oyen, W. J. G. Bonenkamp, J. J.; Boezeman, J. B.; Adema, G. J.; Bulte, J. W. M.; Scheenen, T. W: J.; Punt, C. J. A.; Heerschap, A.; Figdor, C. G. Nat. Biotechnol. 23 (2005) 1407.

Deng, Y. H.; Wang, C. C.; Hu, J. H.; Yang, W. L.; Fu, S. K. Colloids Surf. A: Physicochem. Eng. Aspects 262 (2005) 87.

Desai, S- J.; Singh, P.; Simonelli, A. P.; Higuchi, W. I. J. Pharm. Sci. 55 (1966) 1235.

Dexeus, F. H. Logothetis, C. J. Sella, A. Amato, R. Kilbourn, R. Ogden, S. Striegel, A. Kwan, J. Newman, R. A. J. Urol. 146 (1999) 709.

Dhodapkar, K. M.; Krasovsky, J.; Willamson, B.; Dhodapkar, M. V. J. Exp. Med. 195 (2001) 125.

Dormer, k.; Seeney, C.; Lewelling, K.; Lian, G.; Gibson, D; Johnson, M. Biomaterials 26 (2005) 2061.

Duke, C.; Latham, K.; Williams, C. Zeolites 15 (1995) 213.

Dumitriu, D.; Bârjega, R.; Frunza, L.; Macovei, D.; Hu, T.; Xie, Y.; Pârvulescu, V.; Kaliaguine, S. J. Catal. 219 (2003) 337. 
Duncan, R. Nanotoday (2005) 16.

Duncan, R.; Izzo, L. Adv. Drug Deliv. Rev. 57 (2005) 2215.

El-Arini, S. K.; Leuenberger, H. Int. J. Pharm. 121 (1995) 141.

Eliseev, A. A.; Napolskii, K. S.; Lukashin, A. V.; Tretyakov, Y. D. J. Magn. Magn. Mater. 272 (2004) 1609.

Eliseev, A.A; Gorozhankin, D.F.; Zaitsev, D.D.; Lukashin, A.V.; Knotko, A.V.; Tretyakov, Y.D.; Görnert, P. J. Magn. Magn. Mater. 290 (2005) 106.

Fahmy, T. M.; Fong, P. M.; Goyal, A.; Saltzman, W. M. Mater. Today 8 (2005) 18.

Falabella, E.; Doria, V.; Zanon, F.; Correa, R. Micropor. Mesopor. Mater. 25 (1998) 25 .

Fendler, J. H. Chem. Rev. 87 (1977) 877.

Feng, S.-S.; Chien, S. Chem. Eng. Sci. 58 (2003) 4087.

Fernández-Pacheco, R., Arruebo, M.; Marquina, C. I.; Ibarra, M. R., Arbiol, J.; Santamaría, J. Nanotechnology 17 (2006) 1188.

Fernández-Pacheco, R.; Ibarra, M. R.; Valdivia, J. G.; Marquina, C. I.; Serrate, D.; Romero, M. S.; Gutiérrez, M.; Arbiol, J. Technical Proceedings of the 2005 NSTI Nanotechnology Conference and Trade Show, Vol 1. NSTI Publications, Danville (USA) (2005) 144-147.

Fernández-Pacheco, R.; Marquina, C. I.; Valdivia, J. G.; Gutiérrez, M.; Romero, M. S.; Cornudella, R.; Laborda, A.; Viloria, A.; Higuera, T.; García, A.; García de Jalón, J. A.; Ibarra, M. R. J. Magn. Magn. Mater. 311, (2007) 318. 
Ferrari, M. Curr. Opinion Chem. Biol. 9 (2005) 343.

Flores, G. A.; Liu, J. Eur. Cell. Mater. 3 (2002) 9.

Fréchet, J. M. J.; Gillies, E. R. DDT 10 (2005).

Fréchet, J.M.J. Prog. Polym. Sci. 30 (2005) 844.

Fried, J. R. Polymer Science and Technology, Prentice Hall, Upper Saddle River, NJ, p.51, 1995.

Fu, W.; Yang, H.; Chang, L.; Li, M.; Bala, H.; Yu, Q.; Zou, G. Colloids Surf. A: Physicochem. Eng. Aspects 262 (2005) 71.

Fuji T.; de Groot F. M. F.; Sawatzky G. A.; Voogt F. C.; Hibma T.; Okada K. Phys. Rev. B 59 (1999) 3195.

Funovics, M. A.; Kapeller, B.; Hoeller, C.; Su, H.S.; Kunstfeld, R.; Puig, S.; Macfelda, K. Magn. Reson. Imag. 22 (2004) 843.

Furihata, M.; Ohtsuki, Y.; Ido, E.; Iwata, J.; Sonobe, H.; Araki, K.; Ogoshi, S.; Ohmori, K. Virchows Arch. B Cell Pathol. Incl. Mol. Pathol. 61 (1992) 409.

Gao, X. ; Yu, C.H.; Tam, K.Y.; Tsang, S.C. J. Pharm. Biomed. Anal. 38 (2005) 197.

García Cerdá, L.A.; Montemayor, S.M. J. Magn. Magn. Mater. 294 (2005) 43.

García Fierro, J. L. Mater. Sci. Eng. C 23 (2003) 697.

García-Cerda, L. A.; Torres-García, V. A.; Matutes-Aquino, J. A.; AyalaValenzuela, O. E. J. Alloys Compd. 369 (2004) 148. 
Giri, J.; Thakurta, S.; Bellare, J.; Nigam, A.; Bahadur, D. J. Magn. Magn. Mater. $293(2005) 62$.

Gneveckow, U.; Jordan, A.; Scholz, R.; Brüss, V.; Waldöfner, N.; Ricke, J.; Feussner, A.; Hildebrandt, B.; Rau, B.; Wust, P. Med. Phys. 31 (2004) 1444.

Gonzales, M., Krishnan, K. M. J. Magn. Magn. Mater. 293 (2005) 265.

Gould, P., Nanotoday 1 (2006), 34

Goya, G. F.; Grazú, V.; Ibarra, M. R. Curr. Nanosci. 4 (2008) 1.

Grigorieva, N.A.; Grigoriev, S.V.; Okorokov, A.I.; Eckerlebe, H.; Eliseev, A.A.; Lukashin, A.V.; Napolskii, K.S. Physica E: Low-dimens. Syst. Nanostruct. 28 (2005) 286.

Gupta, P. K.; Hung, C. T.; Rao, N. S. J. Pharm. Sci. 78 (1989) 290.

Häfeli, U.; Pauer, G. J. J. Magn. Magn. Mater. 194 (1999) 76.

Häfeli, U.; Sweeney, S. M.; Beresford, B. A. Nucl. Med. Biol. 22 (1995) 147.

Halasz, I.; Agarwal, M.; Marcus, B.; Cormier, W. Micropor. Mesopor. Mater. 84 (2005) 318 .

Hart, D. N. J. Blood 90 (1997) 3245.

Hart, D. N. J.; Pricket, T. C. R. Cell Immunol. (1993).

Hartung, A.; Lisy, M. R.; Herrmann, K.-H.; Hilger, I.; Schüler, D.; Lang, C.; Bellemann, M. E.; Kaiser, W. A.; Reichenbach, J. R. J. Magn. Magn. Mater. 311 (2007) 454 . 
He, C.; Zhao, N.; Shi, C.; Li, J.; Li, H. Mater. Res. Bull. In press (2008).

Hiergeist, R.; Andrä, W.; Buske, N.; Hergt, R.; Hilger, I.; Kaiser, W. A. J. Magn. Magn. Mater. 293 (2005) 80.

Higuchi, T. J. Pharm. Sci. 55 (1963) 1145.

Higuchi, T. J. Pharm. Sci. 50 (1961) 874.

Högemann, D.; Josephson, L.; Weissleder, R.; Basilion, J. P. Bioconjugate Chem. 11 (2000) 941.

Högemann, D.; Ntziachristos, V.; Josephson, L.; Weissleder, R. Bioconjugate Chem. 13 (2002) 116.

Höpken, U. E.; Lipp, M. Immunity 20 (2004) 244.

Hrusesky, W. J. M.; Von Roemeling, R.; Lanning, R. M.; Rabatin, J. T. J. Clin. Oncol. 8 (1990) 1504.

Huang, X.; Chen, Z. J. Magn. Magn. Mater. 280 (2004) 37.

Huang, X.; Chen, Z. Mater. Res. Bull. 40 (2005) 105.

Huh, Y. M.; Jun, Y. W.; Song, H. T.; Kim, S.; Choi, J. S.; Lee, J. H.; Yoon, S.; Kim, K. S.; Shin, J. S.; Suh, J. S.; Cheon, J. J. Am. Chem. Soc. 127 (2005) 12387.

Hunter, R. J.; Matarese, R. Napper, D. H. Coll. Surf. 7 (1983) 1.

Ibarra, M. R.; Fernández-Pacheco, R.; Valdivia, J. G. "Magnetic Nanoparticles for local drug delivery using Magnetic Implants“. Methods in Molecular Biology, Microfluidics and Nanotechnology series. The Humana Press Inc. (Book chapter) In press (2008). 
Im, S. H.; Herricks, T.; Lee, Y. T.; Xia, Y. Chem. Phys. Lett. 401 (2005) 19.

Ito, T.; Liu, Y. J.; Kadowaki, N. Int. J. Hemat. 81 (2005) 188.

Iturbe, T.; Cornudella, R.; De Miguel, R. Lancet 353 (1999) 2158.

Jaboski, J. M.; Okal, J.; Potoczna-Petru, D.; Krajczyk, L. J. Catal. 220 (2003) 146.

Jain, T. K.; Morales, M. A.; Sahoo, S. K., Leslie-Pelecky, D. L.; Labhasetwar, V. Molec. Pharm. 2 (2005) 194.

Jaulin, N.; Appel, M.; Passirani, C.; Barratt, G.; Labarre, D. J. Drug Target. 8 (2000) 165.

Jewell, J. J.; Harbison, J. P.; Scherer, A.; Lee, Y. H.; Florez, L. T. IEEE J. Quant. Electron. 27 (1991) 1332.

Jiang, J.; Li, L.; Xu, F. Mater. Sci. Eng. A 456 (2007) 300.

Jianrong, C.; Yuqing, M.; Nongyue, H.; Xiaohua, W.; Sijiao, L. Biotech. Adv. 22 (2004) 505.

Jordan, A.; Scholz, R.; Wust, P.; Fähling, H.; Krause, J.; Wlodarczyk, W.; Sander, B.; Vogl, T.; Felix, R. Int. J. Hyperthermia 13 (1997) 587.

Jordan, A.; Scholz, R.; Wust, P.; Schirra, H.; Schiestel, T.; Schmidt, H.; Felix, R. J. Magn. Magn. Mater. 194 (1999) 185.

Josephson, L.; Pérez, J. M.; Weissleder, R. Angew. Chem. Int. Ed. 40 (2001) 3204.

Josephson, L.; Tung, C. H.; Moore, A.; Weissleder, R. Bioconjugate Chem. 10 (1999) 186. 
Jung, J. -S.; Choi, K. -H.; Jung, Y. -K.; Lee, S. H.; Golub, V. O.; Malkinski, L.; O’Connor, C. J. J. Magn. Magn. Mater. 272 (2004) 1157.

Kakeji, Y.; Maehara, Y.; Korenaga, D.; Tsujitani, S.; Haraguchi, M.; Watanabe, A.; Orita, H.; Sugimachi, K. J. Surg Oncol. 52 (1993) 207.

Kala, M.; Bakaj, K.; Sinha, S. Anal. Biochem. 254 (1997) 263.

Kang, Y.; Seo, Y. H.; Lee, C. Bull. Korean Chem. Soc. 21 (2000) 241.

Kantor, A. B.; Gibbons, I.; Miltenyi, S.; schmitz, J. Cell Separation Methods And Applications, Marcel Dekker Inc., New York (1997).

Kato, T. Appl. Biochem. Biotechnol. 10 (1984) 199.

Kim, D. K.; Zhang, Y.; Kher, J; Klason, T.; Bjelke, B.; Muhammed, M. J. Magn. Magn. Mater. 225 (2001) 256.

Klabunde, K. J. Nanoscale Materials in Chemistry. Wiley-Interscience pp.1-13.

Kuznetsov A.; Filippov, V.; Kuznetsov, O.; Gerlivanov, V.; Dobrinsky, E.; Malashin, S. J. Magn. Magn. Mater. 194 (1999) 22.

Kuznetsov A.; Filippov, V.; Kuznetsov, O.; Gerlivanov, V.; Dobrinsky, E.; Malashin, S. J. Magn. Magn. Mater. 194 (1999) 22.

Lau, W.S. Infrared characterization for microelectronics. World Scientific (1999).

Lee, S. P.; Chen, Y. J.; Ho, C. M.; Chang, C. P.; Hong, Y. S. Mater. Sci. Eng. B 143 (2007) 1.

Lee, J.; Isobe, T.; Senna, M. P. J. Colloid Interface Sci. 177 (1996) 490. 
Lee, J.; Jin, S.; Hwang, Y.; Park, J. G.; Park, H. M.; Hyeon, T. Carbon 43 (2005) 2536.

Licha, K.; Olbrich, C. Adv. Drug Delivery Rev. 57 (2005) 1087.

Lind, K.; Kresse, M.; Debus, N. P. J. Drug Target. 10 (2002) 221.

Liu, X.; Ma, Z.; Xing, J.; Liu, H. J. Magn. Magn. Mater. 270 (2004) 1 (a).

Liu, X., Xing, J.; Guan, Y.; Shan, G.; Liu, H. Colloids Surf. A, 238 (2004) 127 (b).

Lu, J.; Yang, S.; Ming Ng, K.; Su, C. H.; Yeh, C. S.; Wu, Y. N.; Shieh, D. B. Nanotechnology 17 (2006) 5812-5820

Lu, X.; Liang, G.; Sun, Z.; Zhang, W. Mater. Sci. Eng. B 117 (2005) 147.

Lübbe, A. S.; Bergemann, C.; Brook, J.; McClure, D. G. J. Magn. Magn. Mater. 194 (1999) 149.

Lübbe, A. S.; Bergemann, C.; Huhnt, W.; Fricke, T.; Riess, H.; Brock, J. W.; Huhn, D. Cancer Res. 56 (1996) 4694.

Lübbe, A. S.; Bergemann, C.; Riess, H.; Schriever, F.; Reichardt, P.; Possinger, K.; Matthias, M.; Dörken, B.; Herrmann, F.; Gürtler, R.; Hohenberger, P.; Haas, N.; Sohr, R.; Sander, B.; Lemke, A.-J.; Ohlendorf, D.; Huhnt, W.; Huhn, D. Cancer Res. 56 (1996) 4686.

Lübbe, A.; Alexiou, C.; Bergemann, C. J. Surgical Res. 95 (2001) 200.

Ma, Z. Y.; Liu, X. Q.; Guan, Y. P.; Liu, H. Z. Colloids Surf. A: Physicochem. Eng. Aspects 275 (2006) 87.

Maaßen, S.; Fattal, E.; Müller, R.H. STP Pharma Sci. 3 (1993) 11. 
Macaroff, P. P.; Simioni, A.R., Lacava Z. G. M. J. Appl. Phys. 99 (2006) 102.

Massart, R.; Cabuil, V. J. Chem.Phys. 84 (1987) 967.

Mayordomo, J. I. Zorina, T.; Storkus, W. J.; Zitvogel, L.; Celluzi, C.; Falo, L. D.; Melief, C. J.; Ildstad, S. T.; Martin Kast, W.; Deleo, A. B.; Lotze, M. T. Nature Med; 1 (1995) 1297.

McLellan, A. D.; Starling, G. C.; Williams, L. A.; Hock, B. D.; Hart, D. N. J. Bone Marrow Transplant (1995).

Mekala, S. R.; Ding, J. J. Alloys Compd. 296 (2000) 152.

Mellman, I., Steinman, R. M. Cell 106 (2001) 255.

Merbach, A. E.; Tóth, E. The Chemistry of Contrast Agents in Medical Magnetic Resonance Imaging. Wiley. Chichester, UK (2001).

Messing, G. L; Zhang, S. C.; Jayanthi, G. V. J. Am. Ceram. Soc. 76 (1993) 2707.

Mickisch, G. H. World J. Urol. 12 (1994) 214.

Missirlis, D.; Kawamura, R.; Tirelli, N.; Hubbell, J. A. Eur. J. Pharm. Sci. 29 (2006) 120.

Moghimi, S. M.; Hunter, A. C. Pharm. Res. 18 (2001) 1.

Mohallem, N. D. S.; Seara, L. M. Appl. Surf. Sci. 214 (2003) 143.

Mohamed, M.; Salama, T. J. Colloid Interf. Sci. 249 (2002) 104.

Monfardini, C.; Veronese, F. M. Bioconjugate Chem. 9 (1998) 418. 
Moore, A; Medarova, Z.; Potthast, A.; Dai, G. P. Cancer Res. 64 (2004) 1821.

Morales, M. P.; González-Carreño, T.; Ocaña, M.; Alonso-Sañudo, M.; Serna, C. J. J. Solid State Chem. 155 (2000) 458.

Morawski, A. M.; Lanza, G. A.; Wickline, S. A. Curr. Opin. Biotech. 16 (2005) 89.

Morimoto, Y. J. Pharmacobio-Dynamics 3 (1980) 264.

Mornet, S. J. Mater. Chem. 14 (2004), 2161.

Moroz, P.; Jones, S. K.; Gray, B. N. Int. J. Hyperthermia 18 (2002) 267.

Nageswari, K.; Banerjee, R.; Gupte, R.V. J Biomater Appl. 13 (1998) 74.

Néel, L. Ann. Geophys. 5 (1949) 99.

Nestlé, F. O.; Alijagic, S.; Gilliet, M.; Sun, Y.; Grabbe, S.; Dummer, R. Nature Med. 4 (1998) 328.

Neuberger, T.; Schöpf, B.; Hofmann, H.; Hofmann, M.; von Rechenberg, B. J. Magn. Magn. Mater. 293 (2005) 483.

Ocón, E.; Pouliquen, D.; Kovaleva, Z. V.; Stepanova, T. P. Lavit, S. G. Kudryavtsev, B. N.; Jallet, P. Lab. Invest. 71 (1994) 895.

Okuhata, Y. Adv. Drug Deliv. Rev. 37 (1999) 121.

Oliveira, L. C. A.; Petkowicz, D. I.; Smaniotto, A.; Pergher, S. B. C. Water Res. 38 (2004) 3699.

Oppelt, A. Physical Principles and Technology of Magnetic Resonance Imaging. Magnetism in Medicine, pp 297-342. Wiley-VCH, Weinheim (2007). 
Pankhurst, Q. A.; Connolly, J.; Jones, S. K.; Dobson, J. J. Phys.D: Appl. Phys. 36 (2003) 167.

Park, S.I.; Lim, J.H.; Kim, J.H.; Yun, H.I.; Kim, C.O. J. Magn. Magn. Mater 304 (2006) 406.

Pirko, I.; Ciric, B.; Gamez, J.; Bieber, A. J.; Warrington, A. E.; Johnson, A. J.; Hanson, D. P.; Pease, L. R.; Macura, S. I.; Rodriguez, M. Faseb J. 18 (2004) 1577.

Popat, K. C.; Sharma, S.; Desai, T. A. J. Phys. Chem. B 108 (2004) 5185.

Prior, B. M.; Yang, H. T.; Terjung, R. L. J. App. Physiol. 97 (2004) 1119.

Pulendran, B. Immunol. Res. 29 (2004) 187.

Quellec, P.; Gref, R.; Perrin, L.; Dellacherie, E. ; Sommer, F. ; Verbavatz, J. M. J. Biomed. Mater. Res. 42 (1998) 45.

Rechenberg, H. R.; Coaquira, J. A. H.; Marquina, C.; García-Landa, B.; Ibarra, M. R.; Benito, A. M.; Maser, W.; Muñoz, E.; Martínez, M. T. J. Magn. Magn. Mater. $226(2001) 1930$.

Reinhart, W.H.; Pleisch, B.; Harris, L. G. Clin. Hemorheol. Microcirc. 32 (2005) 227.

Reis e Sousa, C. Nature Rev. Immunol. 6 (2006) 476.

Richardson, J. C.; Bowtell, R. W.; Madder, K.; Melia, C. D.; Adv. Drug Delivery Rev. 57 (2005), 1191. 
Ritter, J. A.; Ebner, A. D.; Daniel, K. D.; Stewart, K. L. J. Magn. Magn. Mater. 280 (2004) 184.

Roberts, P. L.; Chuang, N.; Roberts, H. C. Eur. J. Rad. 34 (2000) 166.

Rudge, S.; Peterson, C., Vessely, C. J. Contr. Release 74 (2001) 335.

Saebo, K. B. PhD Thesis, Uppsala University, Sweden 2004.

Sallusto, F.; Cella, M.; Danieli, C.; Lanzavecchia, A. J Exp Med. 182 (1995) 389.

Schwartz, B. J.; Simonelli, A. P.; Higuchi, W. I. J. Pharm. Sci. 57 (1968) 274.

Shah, P.; Sohma, M.; Kawaguchi, K.; Yamaguchi, I. J. Magn. Magn. Mater. 247 (2002) 1.

Shelley, S. A. Chem. Eng. Prog. 102 (2006) 43.

Shinkai, M. J. Biosci. Bioeng. 94 (2002) 606.

Shinkai, M.; Yanase, M.; Suzuki, m; Honda, H.; Wakabayashi, T.; Yoshida, J.; Kobayasahi, T. J. Magn. Magn. Mater. 194 (1999) 176.

Siepmann, J.; Peppas, N. A. Adv. Drug Deliv. Rev. 48 (2001) 139.

Silva, J. B.; Diniz, C. F.; Ardinson, J. D.; Persiano, A. I. C.; Mohallem, N. D. S. J. Magn. Magn. Mater. 272 (2004) 1851.

Sobalik, Z.; Tvarůžková, Z.; Wichterlová, B. Micropor. Mesopor. Mater. 25 (1998) 225.

Soo, P. L.; Luo, L. B.; Maysinger, D.; Eisenberg, A. Langmuir 18 (2002) 9996. 
Sorensen, C. M. Chapter Magnetism in Nanoscale Materials in Chemistry. Edited by K. J. Klabunde. Wiley-Interscience pp.169-261.

Sorescu, M.; Diamandescu, L.; Grabias, A. Mater. Chem. Phys. 83 (2004) 354.

Steinfeld, U.; Pauliạ, C.; Kaltzạ, N.; Bergemann, C.; Lee, H.-H. Int. J. Pharmaceutics 311 (2006) 229.

Steinman, R. M. Annu. Rev. Immunol. 9 (1991) 271.

Steinman, R. M.; Cohn, Z. A. J. Exp. Med. 137 (1973) 1142.

Stolnik, S.; Illum, L.; Davis, S.S. Adv. Drug Deliv. Rev. 16 (1995) 195.

Sun, Y.; Duan, L.; Guo, Z.; DuanMu, Y.; Ma, M.; Xu, L.; Zhang, Y.; Gu, N. J. Magn. Magn. Mater. 285 (2005) 65.

Sunderland, C. J.; Steiert, M.; Talmadge, J. E.; Derfus, A. M.; Barry, S. E. Drug. Develop. Res. 67 (2006) 70.

Suzuki, M.; Honda, H.; Kobayashi, T.; Wakabayashi, T.; Yoshida, J.; Takahashi, M. Brain Tumour Pathol. 13 (1996) 127.

Suzuki, M.; Shinkai, M.; Kamihira, M.; Kobayashi, T. Biotechnol Appl. Biochem. $21(1995) 1179$.

Symington, F. W.; Brady, W.; Linsley, P. S. J. Immunol. (1993).

Tamaskar, I.; García, J. A.; Elson, P.; Wood, L.; Mekhail, T.; Dreicer, R.; Rini, B. I.; Bukowski, R. M. J. Urol. 179 (2008) 81.

Tang, N. J.; Zhong, W.; Wu, X. L.; Jiang, H. Y.; Liu, W.; Du, Y. W. Mater. Lett. 59 (2005) 1723. 
Tartaj, P.; Morales, M. P.; Veintemillas-Verdaguer, S.; González-Carreño, T.; Serna, C. J. J. Phys. D: Appl. Phys. 36 (2003) 182.

Thomé, T.; Colaux, J. L.; Louette, P.; Terwagne, G.; J. Electron Spectrosc. Relat. Phenom. 151 (2006) 19.

Thurnher, M.; Radmayr, C.; Ramoner, R.; Ebner, S.; Bock, G.; Klocker, H.; Romani, N.; Bartsch, G. Int. J. Cancer 68 (1996) 1.

Ugelstad, J. Prestvik, W. S.; stenstad, P.; Kilaas, L.; Kvalheim, G. Magnetism in Medicine, Wiley-VCH, Berlin. (1998) pp 473-490.

Vadala, M. L.; Zalich, M. A.; Fulks, D. B.; St. Pierre, T. G.; Dailey, J. P.; Riffle, J. S. J. Magn. Magn. Mater. 293 (2005) 162.

Van Aken, P. A.; Liebscher, B.; Styrsa, V. J. Phys. Chem. Miner. 25 (1998) 323.

Varanda, L.C.; Morales, M.P.; Goya, G. F.; Imaizumi, M.; Serna, C.J.; Jafelicci, M. J. Mater. Sci. Eng. B 112 (2004) 188.

Vicent, M. J.; Duncan, R. Trends Biotech. 24 (2006) 39.

Visca, M.; Matijevic, E. J. Colloid Interf. Sci. 68 (1978) 308.

Vonarbourg, A., Passirani, C.; Saulnier, P.; Benoitet, J. P. Biomaterials 27 (2006), 4356

Weinmann, H. J.; Ebert, W.; Misselwitz, B.; Schmitt-Willich, H. Eur. J. Radiol. 46 (2003) 33 .

Weissleder, R., Elizondo, G.; Wittenberg, J.; Rabito, C. A.; Bengele, H. H.; Josephson, L. Radiology 175 (1990) 489-93 
Widder, K. J.; Morris, R. M.; Poore, G.; Howard, D. P.; Senyei, A. E. Proc. Natl. Acad. Sci. USA 78 (1981) 579.

Widder, K. J.; Senyel, A. E.; Scarpelli, G. D. Proc. Soc. Exp. Biol. Med. 158 (1978) 141.

Wiekhorst, F.; Seliger, C. ; Jurgons, R. J. Nanosci. Nanotechnol. 6 (2006) 3222.

Wilhelm, C.; Billotey, C.; Roger, J.; Pons, J. N.; Bacri, J. C.; Gazeau, F. Biomaterials 24 (2003) 1001.

Williams D.B., Carter C.B Transmission Electron Microscopy: A Textbook for Materials Science. Kluwer Academic / Plenum Publishers. (1996).

Wong, E. Quoted by the Kansas City Star, Monday, November 8 (1999).

Wu Yi, S.; Gong Cheng, X.; Za Zhi, X. Medscape 20 (2003) 463.

Wu, K. H.; Huang, W. C. J. Solid State Chem. 177 (2004) 3052.

Wu, K.H.; Chang, Y.C.; Chang, T.C.; Chiu, Y.S.; Wu, T.R. J. Magn. Magn. Mater. 283 (2004) 380.

Wu, M. Z.; Xiong, Y.; Peng, Z. M.; Jiang, N., Qi, H. P.; Chen, Q. W. Mater. Res. Bull. 39 (2004) 1875.

Xie, Y. ; Kaminski M. D. ; Torno, M. D.; Finck M. R.; Liu, X.; Rosengart, A. J. J. Magn. Magn. Mater. 311 (2007) 376.

Xue, J. M.; Zhou, Z. H.; Wang, J. Mater. Chem. Phys. 75 (2002) 81.

Yagoda, A. Semil. Urol. 7 (1989) 199. 
Yagoda, A.; Abi-Rached, B.; Petrylak, D. Sem. Oncology 22 (1995) 42.

Yan, S.; Geng, J.; Chen, J.; Yin, L.; Zhou, Y.; Zhou, E. J. Cryst. Growth 262 (2004) 415.

Yan, S.; Ling, W.; Zhou, E. J. Cryst. Growth 273 (2004) 226.

Yanase, M.; Shinkai, M.; Honda, H.; Wakabayasahi, T.; Yoshida, J., Kobayasahi, T. Jpn. J. Cancer Res. 89 (1998) 463.

Yellen B. B.; Forbes, Z. G.; Halverson, D. S.; Fridman, G.; Barbee, K. A.; Chorny, M.; Levy, R.; Friedman, G. J. Magn. Magn. Mater. 293 (2005) 647.

Yin, H.; Too, H.P.; Chow, G.M. Biomaterials 26 (2005) 5818.

Yong-Hui, D.; Chang-Chun, W.; Jian-Hua, H.; Wu-Li, Y.; Shou-Kuan, F. Colloids Surf. A: Physicochem. Eng. Aspects 262 (2005) 87.

Yu, W. W.; Chang, E.; Sayes, C. M. Drezek, R.; Colvin, V. L. Nanotechnology 17 (2006) 4483.

Zahr, A. S.; Davis, C. A.; Pishko, M. V. Langmuir 22 (2006) 8178.

Zhang, S.; Dong, D.; Sui, Y.; Liu, Z.; Wang, H.; Qian, Z.; Su, W. J. Alloys Comp. 415 (2006) 257.

Zhou, Z. H.; Xue, J. M.; Chan, H. S. O.; Wang, J. J. Mater. Chem. Phys. 75 (2002) 181.

Zhou, Z. H.; Xue, J. M.; Wang, J. J. Appl. Phys. 91 (2002) 6015.

Zimmermann, U.; Pilwat, G. J. Biosci. 31 (1976) 732. 


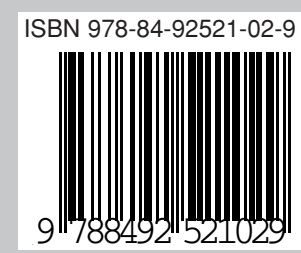

\section{Perey}

Prensas Universitarias de Zaragoza

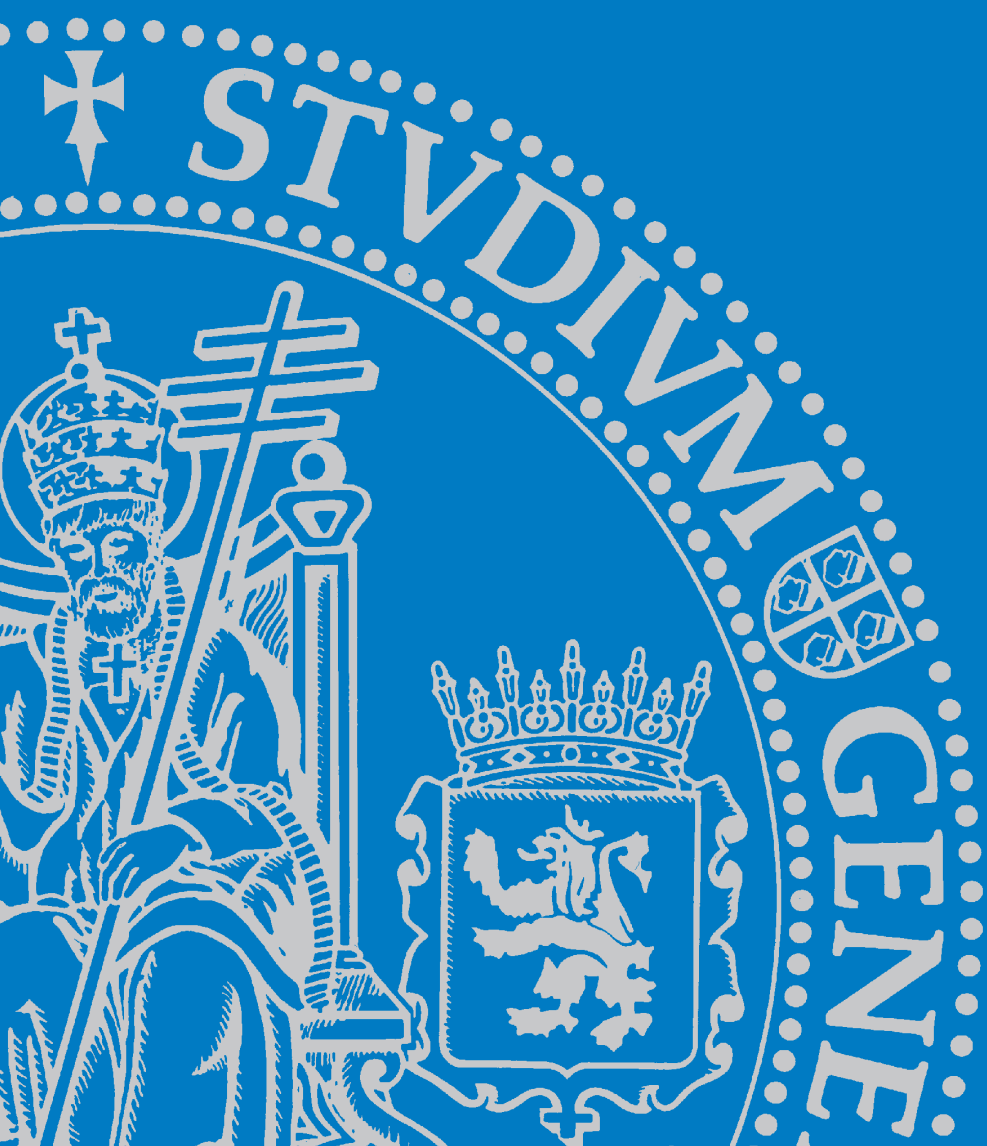

